\title{
Ultrafast electron dynamics measured with a novel time-resolved high-repetition rate momentum microscopy setup
}

\author{
Dissertation \\ for the award of the degree \\ "Doctor rerum naturalium" (Dr.rer.nat.) \\ of the Georg-August-Universität Göttingen
}

within the doctoral program ProPhys

of the Georg-August-University School of Science (GAUSS)

submitted by

Marius Keunecke

from Schwetzingen

Göttingen, 2021 


\section{Thesis Committee}

Prof. Dr. Stefan Mathias

I. Physikalisches Institut, Georg-August-Universität Göttingen

Prof. Dr. Vasily Moshnyaga

I. Physikalisches Institut, Georg-August-Universität Göttingen

\section{Members of the Examination Board}

\section{Reviewer:}

Prof. Dr. Stefan Mathias

I. Physikalisches Institut, Georg-August-Universität Göttingen

\section{Second Reviewer:}

Prof. Dr. Claus Ropers

IV. Physikalisches Institut, Georg-August-Universität Göttingen

\section{Further members of the Examination Board}

Prof. Dr. Vasily Moshnyaga

I. Physikalisches Institut, Georg-August-Universität Göttingen

Prof. Dr. Thomas Weitz

I. Physikalisches Institut, Georg-August-Universität Göttingen

Prof. Dr. Stefan Kehrein

Institut für Theoretische Physik, Georg-August-Universität Göttingen

PD Dr. Martin Wenderoth

IV. Physikalisches Institut, Georg-August-Universität Göttingen

Date of the oral examination: 26.02.2021 


\section{Contents}

$\begin{array}{ll}\text { 1. Introduction } & 1\end{array}$

\begin{tabular}{ll}
\hline 2. Theoretical background & $\mathbf{7}$
\end{tabular}

2.1. Photoemission spectroscopy . . . . . . . . . . . . . . . . . 7

2.2. Light-dressed bandstructures . . . . . . . . . . . . . . . . . . . . . 15

2.3. High-harmonic generation . . . . . . . . . . . . . . . . . . . . . . . . 19

2.4. Static properites of graphene . . . . . . . . . . . . . . . . . 21

2.5. Dynamical properties of graphene . . . . . . . . . . . . . . . . 28

3. Experimental setup 35

3.1. The femtosecond $1 \mathrm{MHz}$ EUV beamline. . . . . . . . . . . . . . . . 35

3.2. The time-of-flight momentum microscope . . . . . . . . . . . . . . . . 41

3.3. Data post-processing . . . . . . . . . . . . . . . . . 47

4. Benchmarking the time-resolved momentum microscopy setup 55

4.1. Introduction . . . . . . . . . . . . . . . . 55

4.2. Real-space resolution . . . . . . . . . . . . . . . . 56

4.3. Static momentum microscopy . . . . . . . . . . . . . . . . . . . . 58

4.4. Time-resolved momentum microscopy . . . . . . . . . . . . . . . . . . 60

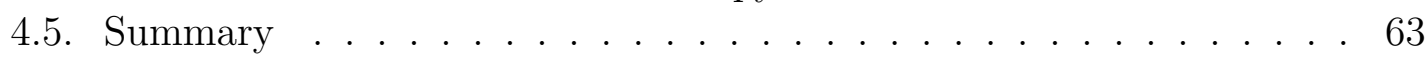

5. Ultrafast Auger recombination in graphene 67

5.1. Introduction . . . . . . . . . . . . . . . 67

5.2. Measurement details . . . . . . . . . . . . . . . . 70

5.3. Results . . . . . . . . . . . . . . . . . . . 70

5.4. Discussion . . . . . . . . . . . . . . . . . . . . . . 78

5.5. Concluding remarks . . . . . . . . . . . . . . . . . . 79

\begin{tabular}{|l|l|}
\hline 6. Electromagnetic dressing of the electron energy spectrum of $\mathrm{Au}(111)$ & \\
\hline \hline at high momenta & 81
\end{tabular}

6.1. Introduction . . . . . . . . . . . . . . . . . . . 81

6.2 . Theoretical description . . . . . . . . . . . . . . 83 
6.3. Measurement details . . . . . . . . . . . . . . . . . 88

6.4. Results . . . . . . . . . . . . . . . . . . . 90

6.5. Discussion . . . . . . . . . . . . . . . . . . . . . . . 94

\begin{tabular}{ll}
\hline 7. Concluding remarks & 99
\end{tabular}

7.1. Summary . . . . . . . . . . . . . . . . . . . . . . . . . 99

7.2. Outlook . . . . . . . . . . . . . . . . . . . . . 101

\begin{tabular}{ll}
\hline Bibliography & 107
\end{tabular}

\begin{tabular}{|l}
\hline A. Supplementary information for chapter 3 \\
\hline
\end{tabular}

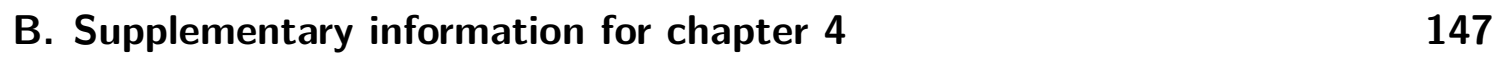

\begin{tabular}{ll}
\hline C. Supplementary information for chapter 5 & 157
\end{tabular}

\begin{tabular}{ll}
\hline D. Supplementary information for chapter 6 & 165
\end{tabular}

\begin{tabular}{lr}
\hline E. List of publications & 171
\end{tabular} 


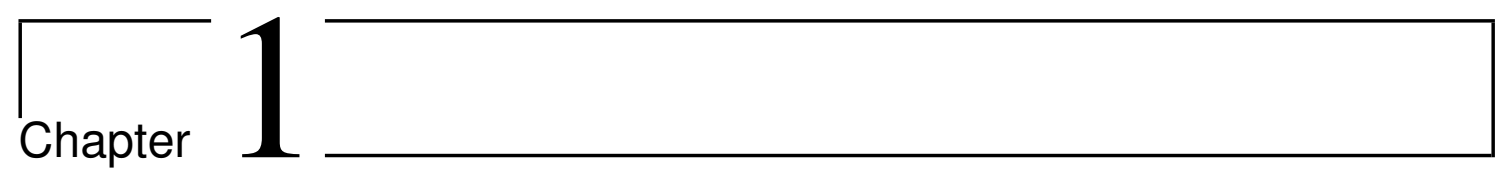

\section{Introduction}

The conversion of energy is one of the most fundamental aspects of physics that we encounter everyday. The efficiency, i.e., the ability to steer available energy into the desired outcome, plays thus a crucial role in every technological area. Strategies to control the energy flow in the desired manner are therefore of fundamental importance. A good example is a solar-cell where an optical excitation is transformed into electrical energy. Here, electron-hole pairs are generated by the absorption of photons and subsequently separated in the p-n junction. The maximum theoretical efficiency of such a solar cell is given by the so-called Shockley-Queisser limit [1] and routes to move beyond it are highly sought-after [2]. Possible mechanisms to do so include:

i) The generation of multiple carriers after absorption of a single photon (carrier multiplication (CM)) induced by, e.g., impact excitation (IE) [3-5], multiple exciton generation (MEG) [6], or singlet fission [9 11].

ii) The slow down of thermalization of the generated carriers towards the band bottom. This can be achieved by trapping the excited carriers in a long-lived state [12 15], i.e., by suppressing available relaxation channels.

All these processes desribed above necessitate an understanding of the optical excitation and subsequent relaxation and dissipation of the charge carriers, which can be described as scattering within and between several interacting subsystems present in the material. These subsystems are commonly described as the orbital-, spin-, lattice-, and charge degrees of freedom (see figure 1.1 (a)).

These subsystems and their interactions do not only determine the microscopic pathway of energy flow (e.g., after optical excitation), but they can also result in effects of technological importance (e.g., superconductivity, colossal magnetoresistance (CMR) and many more). Gaining access to the dynamics in these subsystems and their interactions is therefore of utter scientific importance and necessitates experiments that are able to approach the relevant energy-, length-, and timescales (see $1.1(\mathrm{~b})$ ). 
(a)

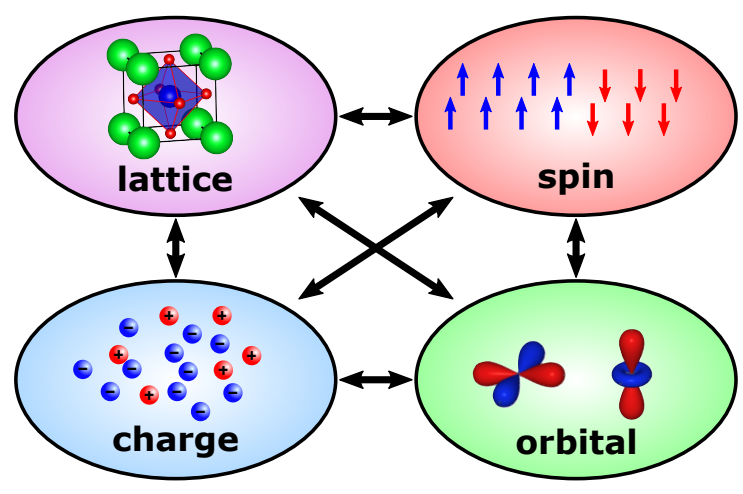

(b)

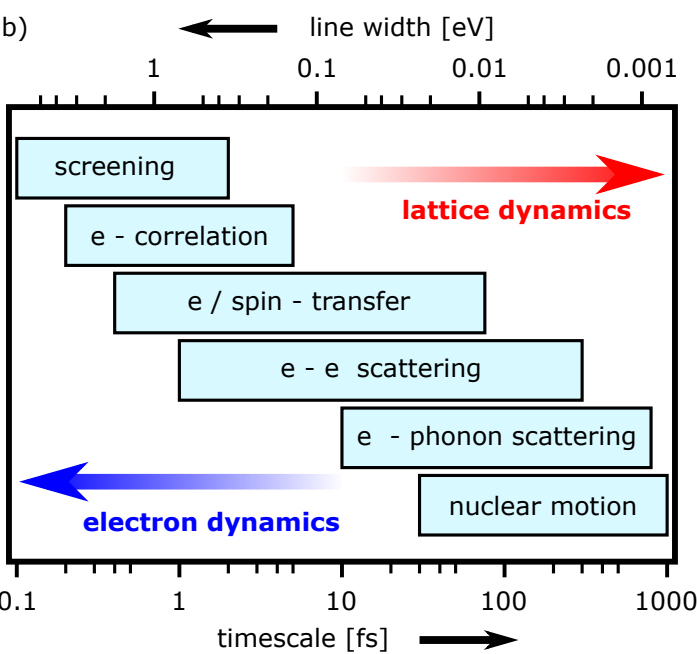

Figure 1.1.: (a) Interplay between the spin- charge- lattice- and orbital degrees of freedom in a material. (b) Typical time scales and respective line widths of excitations and relaxations processes in solid materials. Sketched after [16], whereby the lattice has been rendered using VESTA [17].

In general, pump-probe measurement are considered the method of choice to track dynamics from atto- to nanosecond timescales. Thereby, the material system is subject to an excitation (pump) and subsequent induced changes in it's properties are tracked (probed) dynamically. The plethora of choices for pump and probe results in many different approaches, with their distinctive dis- and advantages, e.g., (without any valuation): time-resolved Raman spectroscopy [18, 19], time-resolved crystallography [20, 21], time-resolved magneto-optical Kerr effect (MOKE) [22, 23, transient absorption spectroscopy [24, 25], ultrafast low energy-electron diffraction (ULEED) [26, 27] and transmission electron microscopy (UTEM) [28, 29], and many more.

The method of choice within this thesis is time- and angle-resolved photoemission spectroscopy (trARPES) which allows to follow an optical excitation and subsequent relaxation within the electronic band structure (i.e., the charge subsystem) of a solid-state material on femto- to attosecond timescales. Thereby, an ultrashort laser pulse is used to excite the material and another time-delayed $(\Delta t)$ pulse is used to photoemit electrons from the sample which are subsequently analyzed. In order to access the full spectroscopic information on the electronic band structure in- and out-of-equilibrium, time-resolved measurement of multiple observables is required, i.e., the photoelectron's energy $(E)$, momentum $\left(\mathbf{k}=k_{x}, k_{y}, k_{z}\right)$, and $\operatorname{spin}(\uparrow, \downarrow)$ are desirable [30, 31].

At present, trARPES experiments mostly rely on hemispherical analysers and are performed utilizing $\mathrm{kHz}$ visible, high-harmonic-generation (HHG) or synchrotron (free-electron laser (FEL)) radiation [32-45], whereby commonly only the energy $(E)$ and one in-plane momentum direction $\left(k_{x}\right.$ or $\left.k_{y}\right)$ within the surface Brilluoin zone can be measured at once. Other detection schemes, employing time-of-flight 
detectors as the ARTOF or THEMIS machines can measure the energy $(E)$ and both in-plane momenta $\left(k_{x}, k_{y}\right)$ simultaneously but are limited due to their comparatively low acceptance angle on the order of $\pm 20^{\circ}$ [46 50].

New electron spectrometers termed momentum microscopes can circumvent this issue and measure the full $k_{x}, k_{y}$ resolved photoelectron distribution with an acceptance angle of $\pm 90^{\circ}$ for typical extreme ultraviolet (EUV) energies. They have been used so far mostly in static (and spin-resolved) bandmapping employing vacuum ultraviolet (VUV), HHG or synchroton radiation [31, 51 63], whereby time-resolved measurements where hindered initially due to the overall low $(\mathrm{kHz})$ repetition rates and thereof resulting signal distortions due to space charge [64 67]. Different energy filtering schemes employing hemispherical or time-of-flight (ToF) devices are in use, whereby ToF necessitates a pulsed and synchronized excitation light source but offers access to all kinetic energies at once, i.e., the full 3D photoelectron distribution $I\left(E, k_{x}, k_{y}\right)$ can be measured.

In terms of light source development, free electron lasers (FELs) and high-harmonic generation (HHG) are promising EUV sources, which offer ultrashort pulses and allow to access the electronic bandstructure on femtosecond or even attosecond timescales within the whole surface Brillouin zone (SBZ) with high $(\mathrm{kHz}-\mathrm{MHz})$ repetition rates [68, 81$]$.

These recent developments in laser technology and electron spectrometers allow nowadays for the build-up of a new generation of trARPES experiments, which grants time-resolved access to the photoelectron's energy $(E)$ and both inplanemomenta $\left(k_{x}, k_{y}\right)$ simultaneously within the whole SBZ as depicted in figure 1.2 .

In this thesis, such a new generation of a trARPES experiment has been successfully built up from scratch combining the advantages of a ToF momentum microscope, i.e., the simultaneous acces to the full 3D photoelectron distribution $I\left(E, k_{x}, k_{y}\right)$, with a lab-based HHG source delivering, femtosecond EUV pulses, and a flexible pump-beamline all operating at the exceptional repetition rate of $1 \mathrm{MHz}$. Thus, this setup allows to track an optical excitation and the subsequent relaxation and dissipation with femtosecond time resolution within the whole 3D surface Brillouin zone.

The first time-resolved momentum microscopy experiments have been demonstrated recently employing FEL radiation [82, 83, visible light (400 nm) within a two-photon-photoemission (2PPE) experiment [84] and within this thesis [77 80] and other works [81, 85, 87]) using a lab-based high harmonic generation (HHG) source, thereby defining the current state-of-the-art in time-resolved momentum microscopy (trMM) worldwide.

The newly built trMM setup has been benchmarked (chapter 4) and successfully applied to study the role of Auger recombination in the electron-electron scattering of graphene (chapter 5), the generation of light-dressed electron energy spectra on a $\mathrm{Au}(111)$ surface (chapter 6), and orbital imaging from a PTCDA monolayer on $\operatorname{Ag}(110)$ [77].

The thesis is structured as follows, in chapter 2 the theoretical background for the upcoming discussion is introduced. Here, a short overview over time- and angle- 


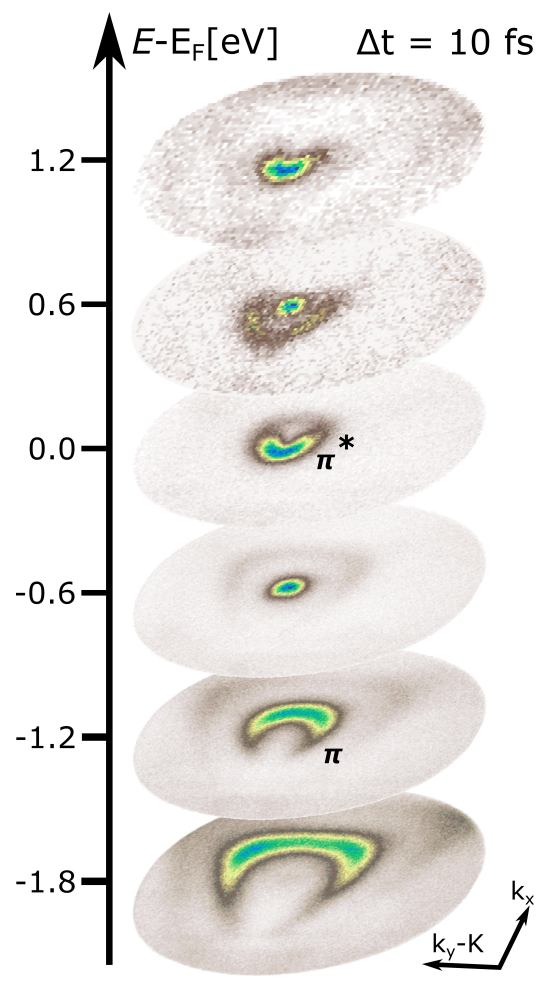

Figure 1.2.: Energy- and in-plane-momentum resolved photoemission data showing the electron dynamics at the K-point (i.e., at the edge of the SBZ) of graphene upon illumination by an infrared femtosecond laser pulse at an time-delay $(\Delta t)$ of $10 \mathrm{fs}$. After [80].

resolved photoemission spectroscopy, light-dressed bandstructures, high-harmonic generation and selected properties of graphene is given.

Subsequently, the experimental setup and data handling is discussed in detail in chapter 3 and benchmarked in chapter 4 with emphasis on the capabilities for trMM.

In chapter 5, to the best of my knowledge, the first direct experimental evidence and quantification of ultrafast Auger recombination in the non-thermal dynamics of graphene is presented. Here it could be shown that highly excited charge carriers are generated by Auger recombination right after optical excitation of n-doped graphene with IR laser pulses on an ultrafast 10 fs timescale.

Floquet engineering is the subject of chapter 6, thereby the material properties are coherently controlled by periodic driving from, e.g., the electric field of a laser pulse [88, 89]. This additional time-periodicity results in the creation of Floquet-states, the temporal analogue of the well-known Bloch states [90, 91]. Such a creation of a light-matter coupled state represents a coherent modification of the material's properties. Commonly, trARPES is seen as the method of choice to identify such light-dressed states, which will results in so called "sidebands", which are replica features of the bandstructure spaced by the driving photon energy $\hbar \omega$. Thereby, the creation of Floquet-(Bloch) states have to be discerned from the laser-assisted 
photoelectric effect (LAPE), i.e., the dressing of the free electron final states in the vacuum, which does not represent a coherent modification of the material but also leads to the generation of sidebands spaced by $\hbar \omega$ [92 95]. In chapter 6 the timedependent light-dressed photoelectron distributions from a $\mathrm{Au}(111)$ crystal have been measured exceeding the first surface Brillouin zone to disentangle the envisaged Floquet-Bloch states from the laser-assisted photoelectric effect (LAPE). Thereby, the screening of the electric field at the vacuum metal interface has been identified to hinder the build-up of Floquet-Bloch bands.

The thesis finishes with a short summary discussing the newly built momentummicroscopy setup and the results gained therewith. Here, the orbital imaging project is briefly mentioned but not further detailed within this thesis. The interested reader is refereed to the published manuscript [77]. Subsequently, not yet realized experimental possibilities are presented and an outlook towards direct control on electron-electron scattering in graphene and realizing Floquet engineering is given. 


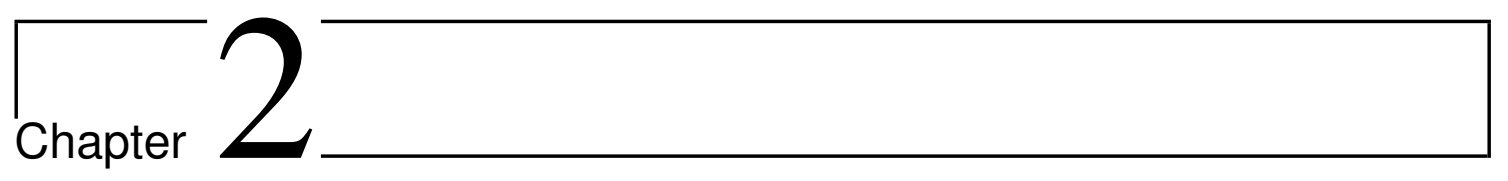

\section{Theoretical background}

Within this chapter, the theoretical background for the upcoming scientific work is outlined. At first, a general description of the main experimental technique, i.e., photoemission spectroscopy (PES), is given. Light-dressed bandstructures as observables in time- and angle-resolved photoemission spectroscopy (trARPES) are introduced subsequently and are further discussed in chapter 6. Afterwards, the extreme ultraviolet (EUV) generation scheme, i.e., the high-harmonic generation (HHG) used for the photoemission spectroscopy is briefly presented. This chapter finishes with the static and dynamic properties of graphene which were investigated in chapters 4 and 5 .

\subsection{Photoemission spectroscopy}

Photoemission spectroscopy (PES) is based on the photoelectric effect [96], which was first observed by Hertz in 1887 [97]. In a simple photoemission experiment monochromatized light is used to photoemit electrons from a sample, which are subsequently analyzed. The kinetic energy of the photoemitted electron upon detection is thereby given by [30]:

$$
E_{k i n}=\hbar \omega-\phi-E_{B}
$$

with the photon energy $\hbar \omega$, the sample's workfunction $\phi$, and the binding energy of the electron $E_{B}$.

Additionally, the electron momentum $\left(\mathbf{k}=k_{x}, k_{y}, k_{z}\right)$ is a desirable observable to gain information on the electronic bandstructure $E_{B}(\mathbf{k})$. Therefore, in angle-resolved photoemission spectroscopy (ARPES) the angles $\theta_{k}, \phi_{k}$, under which the electron leaves the surface, are measured, which are related to the in-plane momentum $k_{\|, i}$ in the direction $\phi_{k}$ of it's initial state by (see section 2.1.2 for additional details):

$$
k_{\|, i}=\frac{1}{\hbar} \sqrt{2 m_{e} E_{k i n}} \sin \left(\theta_{k}\right) .
$$


Thus, the electronic band structure $\left(E, k_{x}, k_{y}\right)$ of the material in equilibrium, i.e., the occupied part of the bandstructure ${ }^{1}$ as given by the Fermi-Dirac distribution, can be investigated. This results in the well known paraboloid $E_{k i n}\left(k_{\|, i}\right)$ describing the photoemission horizon, i.e., the maximum $k_{\|, i}$ that is accessible for a certain binding energy of the electrons $E_{k i n}$ and thus depends on the workfunction $\phi$ and the used photon energy $\hbar \omega$.

a)

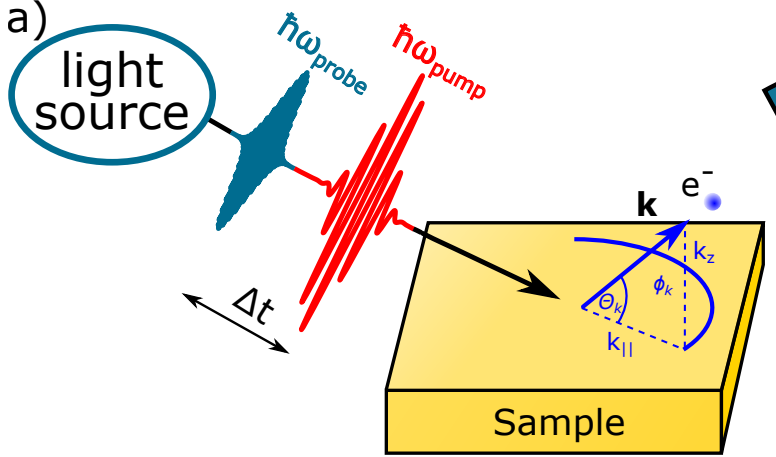

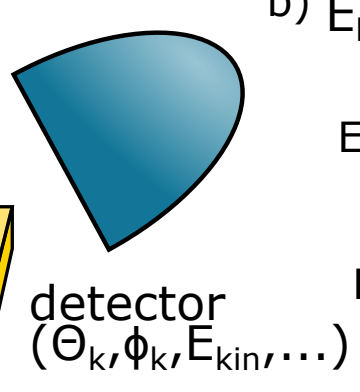

b)

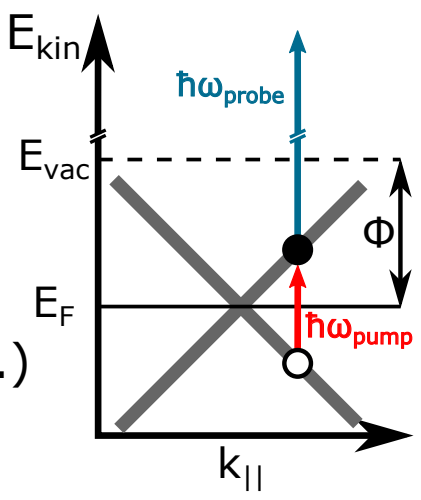

Figure 2.1.: Simplified sketch of a trARPES experiment. a) A photon source delivers pump and probe pulses, which are used to excite (pump) the bandstructure and subsequently photoemit (probe) the electrons within their out-of-equilibrium distribution stroboscopically. Typically, the angles $\theta_{k}, \phi_{k}$ and the kinetic energy $E_{k i n}$ of the photoemitted electrons are measured by the detector, but also additional information (e.g., the spin) can be gathered. b) Excitation diagram sketched for a linearly dispersing bandstructure (grey). The pump (red) photoexcites carriers into the formerly unocuppied region above the Fermi energy $E_{F}$, which can be photoemitted by the time-delayed $(\Delta t)$ probe (blue).

From this simple picture already several important statements can be deduced and connected to the trMM setup built-up in this thesis

i) The used photon energy $\hbar \omega_{\text {probe }}$ determines the maximum accessible $k_{\|, i}$ and $E_{B}$. Moving to higher photon energies, e.g., using extreme ultraviolet light (EUV) as delivered by the high-harmonic generation in this thesis, allows to access the whole surface Brillouin zone (SBZ) and deeper lying electronic states (higher $E_{B}$ ).

ii) The acceptance angle, i.e., the accessible range for $\theta_{k}$ of the used photoelelectron detector, restricts the visible part of the photoemission horizon, whereby the momentum microscope allows to measure $\theta_{k}= \pm 90^{\circ}$ for the used photon energies within this thesis (compare section 3.2).

1 Strictly speaking, only the spectral function $A\left(\epsilon_{i}, k_{i}\right)$ is directly measured (see section 2.1.1. Thus, for introductory purpose, we assume here a non-interacting system. 
iii) The parallel detection scheme of the momentum microscope allows to measure $k_{\|, i}$ for all $\phi_{k}=0-360^{\circ}$ simultaneously. Thus the full photoemission horizon is directly imaged, whereas common hemispherical analysers are restricted to certain $\phi_{k}=$ const. therefore requiring a rotation of the measurement geometry.

So far only static ARPES has been discussed, i.e., the equilibrium bandstructure as defined by the Fermi-Dirac distribution, is probed. To access the unoccupied part of the bandstructure, i.e., the out-of-equilibrium bandstructure, a pump-probe scheme is used within this thesis and now further detailed, but also other possibilities exist, see, e.g., inverse photoemission spectroscopy (IPES) [30].

In a pump-probe photoemission measurement (see figure 2.1 a)) a light source is used to deliver pump laser pulses to excite the sample, and time-delayed $(\Delta t)$ probe pulses are used to measure the subsequent changes in the electronic bandstructure stroboscopically. The pump thereby brings the material into an excited state which can, but does not have to, involve an electronic dipole transition as depicted in figure $2.1 \mathrm{~b}$ ). Subsequently, the probe pulse photoemitts electrons from the outof-equilibrium state and via shifting the controlled time-delay $(\Delta t)$ snapshots of the electronic bandstructure during excitation, relaxation, and dissipation can be measured.

Commonly, the pump photon energy is chosen such that it is much smaller in comparison to the workfunction of the material $\left(\hbar \omega_{\text {pump }} \ll \phi\right)$ to prevent a strong background by pump-induced photoelectrons (due to multi-photon photoemission).

First pump-probe photoemission measurements have been performed in the 1980s [98, 99] within the framework of time-resolved two-photon photoemission (tr2PPE) and thereby laid the foundation for the interpretation of trARPES, pioneered by Haight et al. [100, 101], and trMM spectra.

In the following the main theoretical aspects of photoemission will be presented, a more detailed discussion can be found in the respective literature (see, e.g., [30, 102 107]).

\subsubsection{Photoemission theory}

In general the photoemission process can be described by Fermi's golden rule between the $\mathrm{N}$ electron intial- $\Phi_{i}^{N}$ and final states $\Phi_{f}^{N}$ with the transition rate $w_{f i}$ [30]:

$$
w_{f i}=\frac{2 \pi}{\hbar}\left|\left\langle\Phi_{f}^{N}\left|H_{i n t}\right| \Phi_{i}^{N}\right\rangle\right|^{2} \delta\left(E_{f}^{N}-E_{i}^{N}-\hbar \omega\right),
$$

and a small perturbation by the interaction Hamiltonian $H_{\text {int }}$ :

$$
H_{i n t}=\frac{e}{2 m c}\left(\mathbf{A} \cdot \hat{p}+\hat{p} \cdot \mathbf{A}+\frac{e^{2}}{2 m c^{2}} \mathbf{A}^{2}\right) \approx \frac{e}{m c} \mathbf{A} \cdot \hat{p},
$$

here $\mathbf{A}$ is the vector potential of the incident electromagnetic wave, $\hat{p}=i \hbar \vec{\nabla}$ is the momentum operator and the $\delta$-function ensures energy conservation upon the 
transition from the initial energy $E_{i}^{N}$ to the final energy $E_{f}^{N}$ of the N-electron state. The commutation relation leads to $(\mathbf{A} \cdot \hat{p}+\hat{p} \cdot \mathbf{A}=2 \mathbf{A} \cdot \hat{p}+i \hbar(\vec{\nabla} \cdot \mathbf{A}))$, whereby commonly the terms $i \hbar \vec{\nabla} \cdot \mathbf{A}$ (surface photoemission; see, e.g., [108]) and $\mathbf{A}^{2}$ (two photon process) are neglected. Additionally, for typical wavelengths $\lambda$ used in photoemission the dipole approximation is justified since it is much larger compared to the inter atomic distances.

Commonly, photoemission is discussed within in the so-called sudden approximation (for a discussion beyond the sudden approximation see, e.g., [109]), which assumes that the response of the system to the creation of the photoinduced hole is instantaneous and that the interaction of the escaping photoelectron and the remaining system is negligible. Thus the final state $\left|\Phi_{f}^{N}\right\rangle$ can be written as [30]:

$$
\left|\Phi_{f}^{N}\right\rangle=\left|\phi_{f, E, k_{f}}\right\rangle \sum_{s}\left|\Phi_{f, s}^{N-1}\right\rangle
$$

where $\sum_{s}\left|\Phi_{f, s}^{N-1}\right\rangle$ are the s final states of the interacting (N-1) remaining electrons within the system and $\left|\phi_{f, E, k_{f}}\right\rangle$ is the single-particle wavefunction of the photoemitted electron with energy $E$ and momentum $k_{f}$. This can be viewed as the readjustment of the (N-1) electrons in s different configurations to minimize the overall energy upon creation of the photohole. In a similar way, one can use a single-particle view for the initial state $\left|\Phi_{i}^{N}\right\rangle$, i.e.:

$$
\left|\Phi_{i}^{N}\right\rangle=\left|\phi_{i, k_{i}}\right\rangle\left|\Phi_{i}^{N-1}\right\rangle
$$

with the single-particle initial state wavefunction $\left|\phi_{i, k_{i}}\right\rangle$. The photocurrent $I$ (intensity) of electrons with kinetic energy $E$ and momentum $k_{f}$ reaching the detector can be written to:

$$
\begin{aligned}
I\left(E, k_{f}\right) \propto & \sum_{i}\left|\left\langle\phi_{f, E, k_{f}}\left|H_{\text {int }}\right| \phi_{i, k_{i}}\right\rangle\right|^{2} \sum_{s}\left|\left\langle\Phi_{f, s}^{N-1} \mid \Phi_{i}^{N-1}\right\rangle\right|^{2} \\
& \underbrace{\delta\left(E_{f}^{N}-E_{i}^{N}-\hbar \omega\right)}_{\text {energy conservation }} \underbrace{\delta\left(E-E_{f}^{N}+\phi\right)}_{\text {detection }},
\end{aligned}
$$

whereby the summation is over all possible initial states $\mathrm{i}$ and excited final states $\mathrm{s}$ of the (N-1) electron system. This equation can be further simplified with definition of the matrix element $\left|M_{f i}\right|^{2}[30$ :

$$
\left|M_{f i}\right|^{2}=\left|\left\langle\phi_{f, E, k_{f}}\left|H_{i n t}\right| \phi_{i, k_{i}}\right\rangle\right|^{2}
$$

and:

$$
\sum_{s}\left|\left\langle\Phi_{f, s}^{N-1} \mid \Phi_{i}^{N-1}\right\rangle\right|^{2}=A\left(\epsilon_{i}, k_{i}\right) f\left(\epsilon_{i}\right)
$$


with the definition of the spectral function $A\left(\epsilon_{i}, k_{i}\right)$ and the distribution function $f\left(\epsilon_{i}\right)$, which are evaluated at the photoelectron's initial state energy $\epsilon_{i}$. In thermal equilibrium the distribution function $f\left(\epsilon_{i}\right)$ is given by the Fermi-Dirac distribution. In total the photocurrent can be written as:

$$
I\left(E, k_{f}\right) \propto \sum_{i}\left|M_{f i}\right|^{2} f\left(\epsilon_{i}\right) A\left(\epsilon_{i}, k_{i}\right) \underbrace{\delta\left(E_{f}^{N}-E_{i}^{N}-\hbar \omega\right)}_{\text {energy conservation }} \underbrace{\delta\left(E-E_{f}^{N}+\phi\right)}_{\text {detection }},
$$

i.e., the measured photocurrent is directly related to the spectral function $A\left(\epsilon_{i}, k_{i}\right)$ of the sample, weighted by the matrix element $\left|M_{f i}\right|^{2}$ and the distribution function $f\left(\epsilon_{i}\right)$, which in principle can all show a time-dependence after excitation [110]. Commonly today, time-reversed LEED states are used in the description of the photoemission process for the final state in a one-step model (see, e.g., [104 106]), which give good results compared to experimental band mapping. Within this thesis however, the focus lies on the excitation and subsequent relaxation of the electronic system and no high-resolution band mapping is performed. Therefore, throughout this thesis the simpler three-step model is used, which is presented in the subsequent section.

\subsubsection{The three step model}

Berglund and Spicer have developed the so-called three-step model [111], which is used for the interpretation of photoemission experiments. The main idea of the three-step model is depicted in figure 2.2, whereby the photoemission process is divided into three independent parts, which will be briefly discussed in the subsequent sections:

(1) The photoexcitation of an electron within the solid by an absorbed photon.

(2) The propagation of the quasi-free electron towards the surface.

(3) The transition of the electron into the vacuum. 


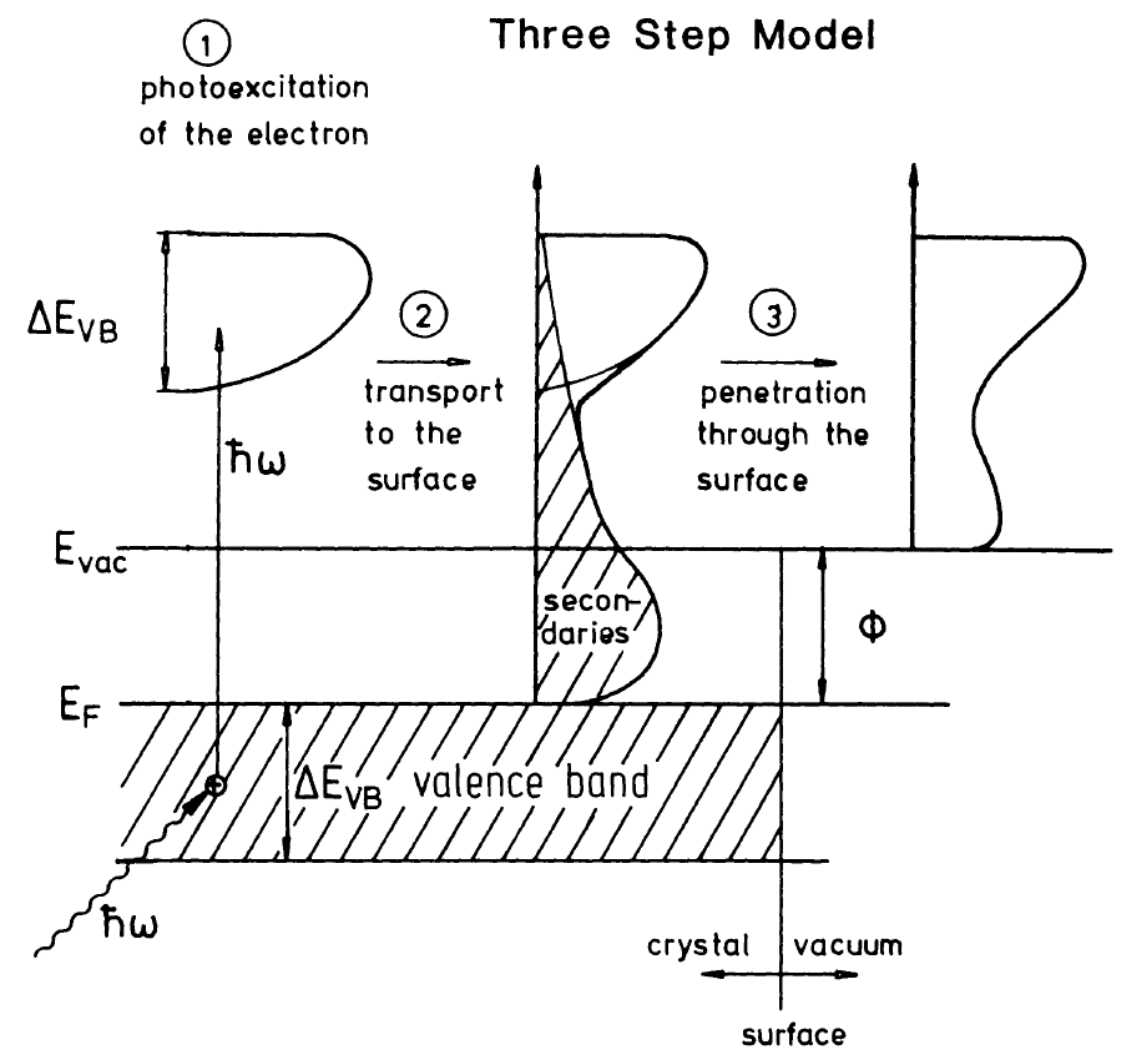

Figure 2.2.: Schematic depiction of the three step model, whereby the photoemission process is divided into three independent steps. (1) The photoexcitation within the solid by a photon of energy $\hbar \omega$. (2) Transport of the excited electron towards the surface, thereby the measured spectrum changes due to the concomitant build up of secondaries as shown. (3) If the momentum of the electron perpendicular to the surface is high enough to overcome the work function $\phi$ it refracts and escapes into the vacuum. Reprinted/adapted by permission from Springer Nature Customer Service Centre GmbH: Springer Nature, Photoelectron Spectroscopy by Stefan Hüfner (C)(2003) [30]. 


\section{1st Step: Photoexcitation}

The photoexcitation is again modelled with Fermi's golden rule, assuming a small pertubation by the interaction Hamiltonian $H_{i n t} \approx \frac{e}{m c} \mathbf{A} \cdot \hat{p}$. The matrix element is now evaluated between initial- $\left(\phi_{i}^{\text {Bloch }}\right)$ and final-Bloch states $\left(\phi_{f}^{\text {Bloch }}\right)$ within the solid to:

$$
\left|M_{f i}\right|^{2}=\left|\left\langle\phi_{i}^{\text {Bloch }}\left|H_{\text {int }}\right| \phi_{f}^{\text {Bloch }}\right\rangle\right|^{2} .
$$

\section{2nd step: Propagation}

The excited electrons travel towards the surface, whereby concomitant elastic and inelastic scattering events occur resulting in a tail of secondaries (scattered) electrons. Therefore, the inelastic mean free path of the electrons gives rise to the surface sensitivity of PES, especially in the UV/EUV regime (see figure 2.3). One can notice that for a significant range of kinetic energies the mean free path is only on the order of several $\AA$ and quite material independent ${ }^{2}$, meaning that with EUV light only the surface can be probed without a significant contribution by secondary (scattered) electrons. The universal behaviour of the mean free path is related to the binding energy of the electrons, i.e., for $E_{k i n} \gg E_{B}$ the electrons can be approximately described by a free-electron gas, resulting in a similar (electron-electron)-scattering behaviour and thus a comparable mean free path [30].

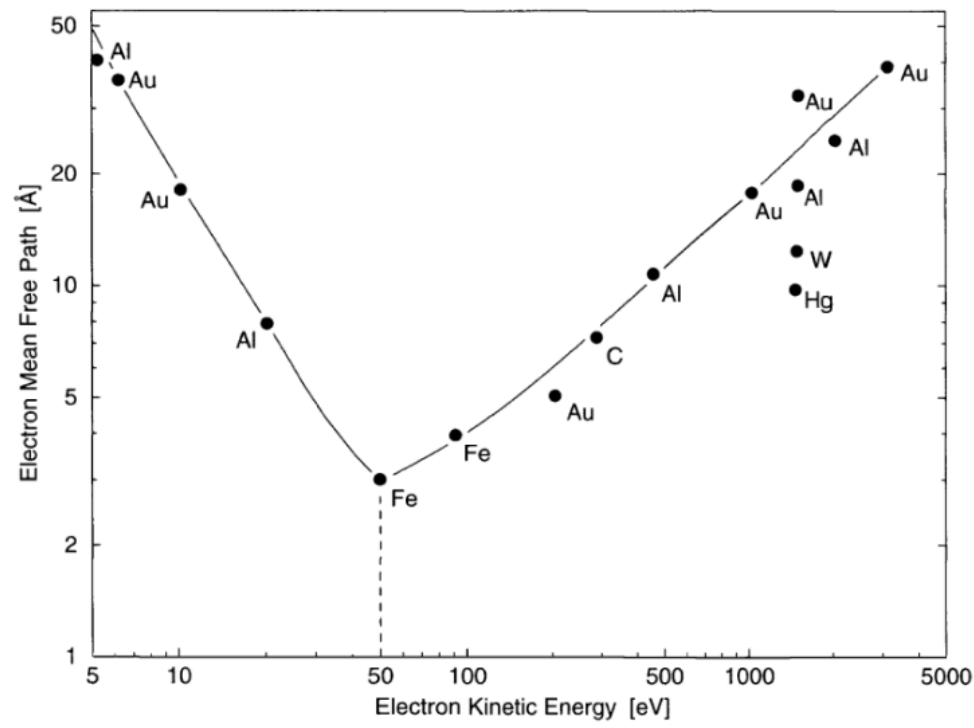

Figure 2.3.: Electron mean free path as a function of their kinetic energy for various materials. The data shows a minimum around kinetic energies $E_{k i n}$ of $50-100 \mathrm{eV}$ on the order of 2-5 . Reprinted/adapted by permission from Springer Nature Customer Service Centre GmbH: Springer Nature, Photoelectron Spectroscopy by Stefan Hüfner @(2003) [30].

\footnotetext{
2 Therefore, the electron mean free path is sometimes referred to the universal curve.
} 


\section{3rd step: Penetration}

If the electron's momentum perpendicular to the surface $k_{\perp}$ is sufficiently high to overcome the workfunction $\phi$ the electron refracts and escapes into the vacuum [11] (otherwise it will be totally reflected). The work function thereby depends on the sample's surface chemistry and the present interactions (see, e.g., [112 115]). Within this process the in-plane momentum vector $k_{\|}$is conserved up to an reciprocal lattice vector $G_{\|}$due to the translational invariance along the surface. This process is depicted in figure 2.4 and allows in ARPES measurements to discern the in-plane momentum $k_{\|, i}$ and energy information of the formerly bound electrons, after:

$$
k_{\|, i}=\frac{1}{\hbar} \sqrt{2 m_{e} E_{k i n}} \sin (\theta),
$$

with the kinetic energy of the free electron in the vacuum $E_{k i n}$. The conservation of the in-plane momentum results also in the so-called photoemission horizon, which depicts the maximal in-plane momentum for a given kinetic energy. This can easily be seen if we rearrange eq. 2.12 and use $k_{\|, i}=\sqrt{k_{x, i}^{2}+k_{y, i}^{2}}$ as well as $\sin \left(\theta=90^{\circ}\right)=1$ :

$$
E_{k i n}\left(k_{x, i}, k_{y, i}\right)=\frac{\hbar^{2}\left(k_{x, i}^{2}+k_{y, i}^{2}\right)}{2 m_{e}}
$$

resulting in the well known paraboloid describing the photoemission horizon. One has to note that since $k_{\perp}$ is not conserved across the surface it's determination is not straightforward but can be done by different methodes (see, e.g., [30, 116-119]).

a)

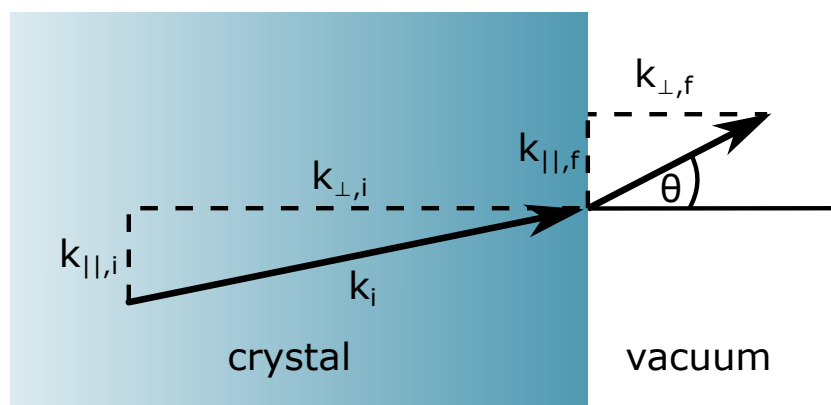

b)

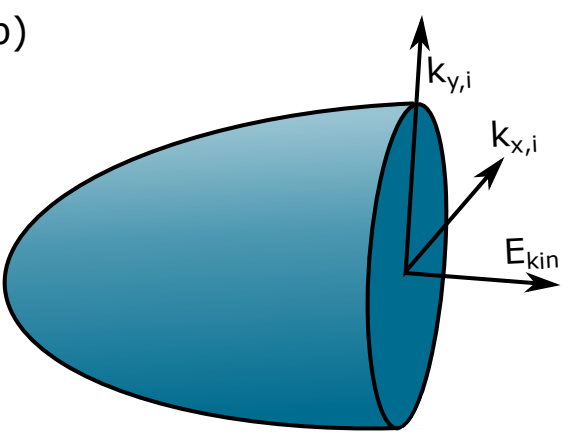

Figure 2.4.: a) Momentum relations at the crystal-vacuum interface, whereby the momentum component parallel to the surface is conserved up to an reciprocal lattice vector $G_{\|}$due to the translational invariance. This allows to connect the measured angle $\theta$ with the momentum information $k_{\|, i}$ in the initial state of the measured electron (eq. 2.12). b) Schematic depiction of the photoemission horizon (paraboloid), which shows the maximum $k_{\|, i}$ for a given kinetic energy $E_{k i n}$. 


\subsection{Light-dressed bandstructures}

Femtosecond engineering of bandstructures and subsequent related properties in condensed matter can be envisaged by so-called Floquet engineering [88, 89]. Thereby, the quantum system is shaped by a periodic driving of, e.g., an intense laser pulse, which results in Floquet states (the temporal analogue of the well known Bloch states) [90, 91]. Here trARPES is the method of choice to probe such light-dressed bandstructures, due to its capability to map out-of-equilibrium bandstructures on femto- to attosecond timescales. For a clear identification of Floquet-(Bloch) bands, the laser-assisted photoelectric effect (LAPE) has to be considered additionally [92, 93. since both effects lead to replica photoemission lines, whereby only the Floquet(Bloch) bands represent a coherent modification of the quantum system (compare figure 2.5). In this section a brief introduction will be given to Floquet physics and LAPE induced by a driving infrared (IR) light pulse, which was used throughout this thesis. A more detailed description can be found in chapter 6, where the scattering between LAPE and Floquet sidebands and the effect of screening is modelled for a $\mathrm{Au}(111)$ crystal.

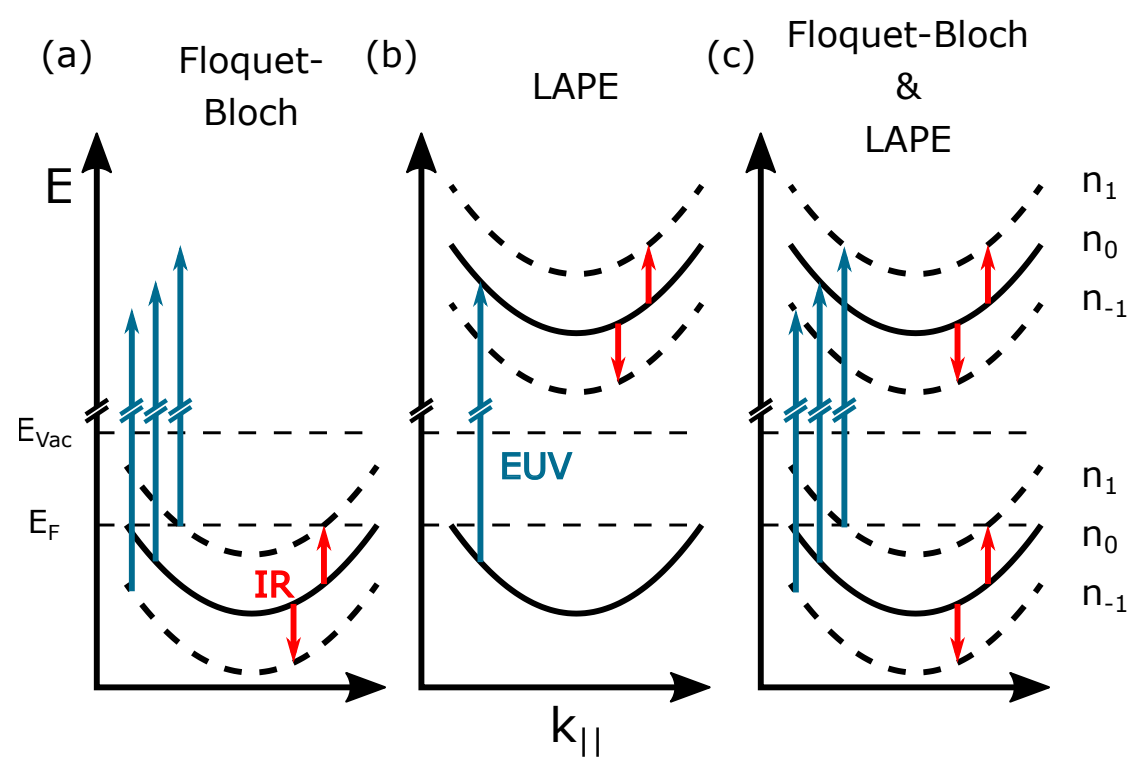

Figure 2.5.: Schematics for the electromagnetic dressing with IR light of (a) Bloch bands, yielding Floquet-Bloch bands, and (b) quasi-free electrons, leading to LAPE. In both scenarios, sidebands $\left(n_{ \pm 1}\right.$, dashed line) of the main photoemission spectral feature $\left(n_{0}\right.$, solid line) are observed in the photoemission experiment. (c) Both processes terminate at the same final state energy, requiring the consideration of scattering amplitude between both processes. Taken from [79]. 


\subsubsection{Floquet theory}

Within this brief discussion of Floquet theory the concept of sidebands, as observable by trARPES, should be introduced. The interested reader is referred to additional articles on the subject (see, e.g., [93, 120 125]). The starting point of the discussion is a time periodic Hamiltonian $H$ with period $T$ describing the light-matter coupled system:

$$
H(t)=H(t+T) .
$$

Floquet theory states that steady state solutions to the time-dependent Schrödinger equation:

$$
H(t)|\psi(t)\rangle=i \hbar \partial_{t}|\psi(t)\rangle
$$

can be written as [121:

$$
|\psi(t)\rangle=\left|u_{\alpha}(t)\right\rangle e^{-\frac{i}{\hbar} \epsilon_{\alpha} t} .
$$

These states $\psi(t)$ are the so-called Floquet states with eigenvalues (quasienergies) $\epsilon_{\alpha}$ and time-periodic Floquet modes $\left|u_{\alpha}(t)\right\rangle=\left|u_{\alpha}(t+T)\right\rangle$. Note the similarity to a Bloch state [126] $\psi_{\text {Bloch }}(r)=e^{i k r} u(r)$, where $u(r)=u(r+R)$ is periodic with respect to a lattice vector $R$.

The quasienergies $\epsilon_{\alpha}$ are only uniquely defined up to integer multiples $n$ of $\hbar \omega$, with $\omega=\frac{2 \pi}{T}$ (Note that within Floquet theory one often also find $\Omega$ instead of $\omega$.), since:

$$
u_{\alpha^{\prime}}=e^{-i n \omega t} u_{\alpha}=u_{\alpha n}
$$

yields the identical solution with shifted quasienergy $\epsilon_{\alpha^{\prime}}=\epsilon_{\alpha}+n \hbar \omega=\epsilon_{\alpha n}$. Thus all solutions can be mapped into a first Floquet- or Brillouin zone obeying $-\hbar \omega / 2 \leq$ $\epsilon_{\alpha} \leq \hbar \omega / 2$. The quasienergy spectrum can then be copied at intervals of $\hbar \omega$ to yield the full quasienergy spectrum. The solutions $|\psi(t)\rangle$ can be written in a discrete Fourier series, so that [122, 123]:

$$
|\psi(t)\rangle=\sum_{n} e^{-i\left(\epsilon_{\alpha}+n \hbar \omega\right) t / \hbar}|n\rangle,
$$

whereby the states $|n\rangle$ are determined by solving the corresponding eigenvalue equation:

$$
\sum_{m}\left(H_{n-m}-n \hbar \omega \delta_{n, m}\right)|m\rangle=\epsilon_{\alpha}|n\rangle, \text { with } \quad H_{n}=\int_{0}^{T} \frac{d t}{T} e^{i n \omega t} H(t) .
$$

From equation 2.18 an intuitive picture can be gained [123], i.e., the steady state solutions $|\psi(t)\rangle$ can be written as a linear combination of states with energies $\epsilon_{\alpha}+n \hbar \omega$ and probability (or occupation) $\langle n \mid n\rangle$. Further insight can be gained from a schematic picture within the 2D Dirac Hamiltonian (compare figure 2.6). Following the work by Farrel et al. [123]: We start from a periodic driving with frequency $\omega$ but negligible amplitude, i.e., the electrons are in the eigenstate of the static Hamiltonian. Turning on the periodic driving leads the electrons to develop probability 
to emit/absorb photons and their formerly unit probability of being found in a certain Floquet zone is smeared out into adjacent Floquet zones. These copies of the formerly undriven bandstructure with seperations of $\hbar \omega$ are theso-called sidebands that can be observed in trARPES [123]. Interesting effects occur when states of different photon order $n$ hybridize, which can lead to band gap openings and allows for Floquet engineering of quantum material.

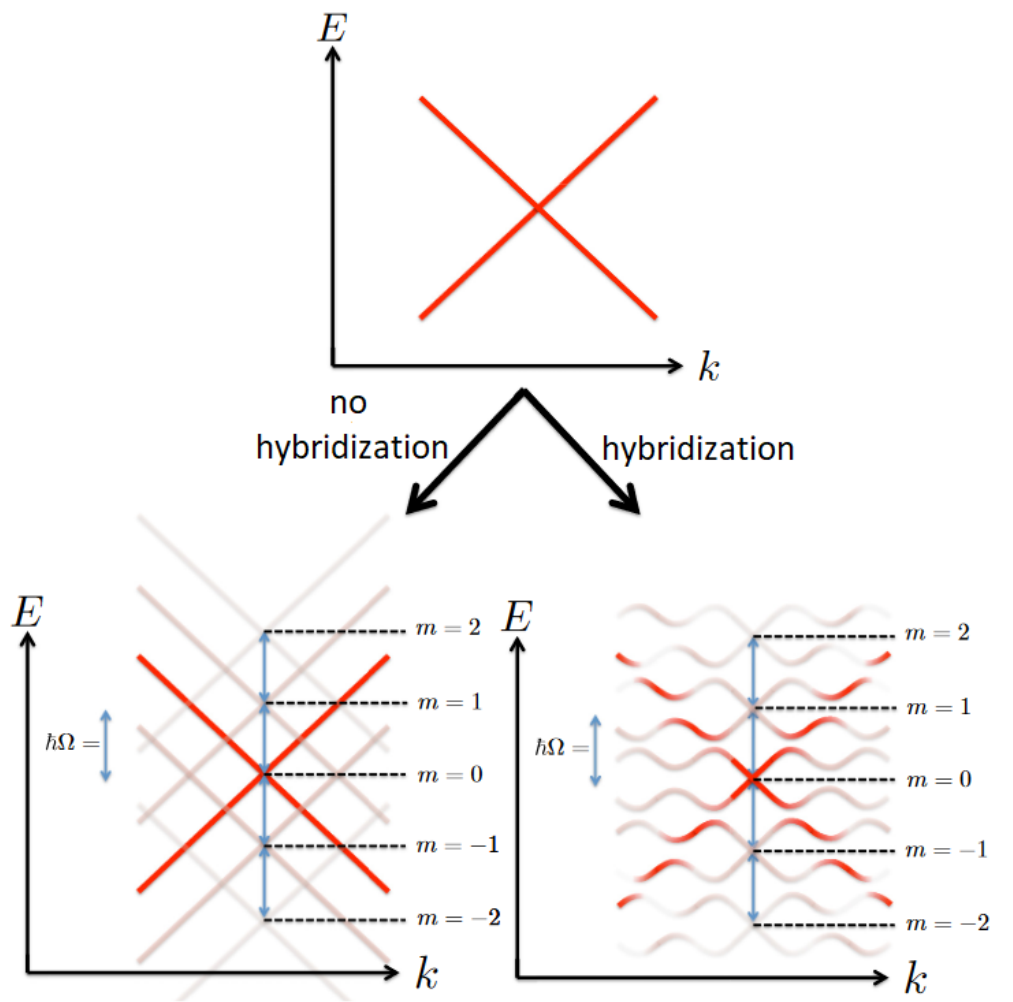

Figure 2.6.: Upon driving, the original Dirac cone is split into sidebands (corresponding to different Floquet zones), whereby their probability is color coded (less intense $=$ less probability). If there is no hybridization between the different photon orders only sidebands appear with separation $\hbar \Omega=\hbar \omega$. On the other hand if they hybridize, the bandstructure is modified and additional band gaps open up. Adapted with permission from Ref. [123]. Copyrighted by the American Physical Society. 


\subsubsection{Laser-assisted photoelectric effect}

The laser-assisted photoelectric effect (LAPE) is a final state effect in photoemission [94, 95, 127], which leads to the generation of so-called sidebands, i.e., replicas of the main photoemission lines separated by the driving light photon energy. From a theoretical point of view it can be understood by considering a Volkov wave $\phi_{V}(\mathbf{r}, t)$ [128] as the final state in photoemission [93, 95, 129, 130]:

$$
\phi_{V}(\mathbf{r}, t)=\frac{1}{(2 \pi)^{3 / 2}} e^{i \mathbf{k r}} e^{-\frac{i}{\hbar}\left(\hbar \omega_{f}+U\right) t} \sum_{n=-\infty}^{\infty} e^{-i n \omega_{I R} t} J_{n}\left(\alpha, \frac{U_{P}}{2 \hbar \omega_{I R}}\right)
$$

with the ponderomotive potential $U_{P}=\frac{e^{2} E_{0}^{2}}{4 m_{e} \omega_{I R}^{2}}$, the eigenenergy of the state $\hbar \omega_{f}$, the generalized Bessel function $J_{n}$ and the LAPE parameter $\alpha$ :

$$
\alpha=\frac{e}{m_{e} \omega_{I R}^{2}} \mathbf{E} \cdot \mathbf{k}
$$

For the typical IR driver conditions used throughout this thesis the pondermotive potential is only on the order of $10 \mathrm{meV}$ is therefore neglected in the following ${ }^{3}$. The transition from an initial state to a final state in photoemission is here calculated within first-order time-dependent perturbation theory (Born approximation [93, 129]) employing a scattering matrix description. The transition rate from an initial state $\phi_{i}=\phi_{i}(\mathbf{r}) e^{-\frac{i}{\hbar}\left(\hbar \omega_{i} t\right)}$ to a final Volkov state reads:

$$
\begin{aligned}
\left(S^{B}-1\right)_{f i} & =-\frac{i}{\hbar} \int_{-\infty}^{\infty} d t\left\langle\phi_{V}|\mathbf{A} \cdot \hat{p}| \phi_{i}\right\rangle \\
& =\frac{2 \pi i}{\hbar} M_{f i} \sum_{n} J_{n}(\alpha) \underbrace{\delta\left(\omega_{f}-\omega_{i}-n \omega_{I R}-\omega_{E U V}\right)}_{\text {energy conservation }},
\end{aligned}
$$

with the photoemission matrix element $M_{f i}=\left\langle\phi_{f}(\mathbf{r})|\mathbf{A} \cdot \hat{p}| \phi_{i}(\mathbf{r})\right\rangle$ generated by the spatial parts of the wavefunction. The photoemission intensity of the $\mathrm{n}$-th sideband is thereby given as:

$$
I_{n} \propto\left|M_{f i}\right|^{2} J_{n}(\alpha)^{2} .
$$

Note that the Bessel function obeys: $J_{-n}(\alpha)=(-1)^{n} J_{n}(\alpha)$ for integer $n$ so that the intensity of the sidebands $I_{n}=I_{-n}$. Moreover, one can approximate the Bessel function $J_{1}$ for small parameters $|\alpha| \ll \sqrt{2}$ resulting in:

$$
J_{1} \sim \frac{1}{2} \alpha
$$

One can directly see that the intensity of the first sideband follows directly the intensity (compare 2.21) of the driving light pulse and scales with $\frac{1}{\omega_{I R}^{4}}$. Thus, it can be used in a trARPES experiment to estimate the cross-correlation between

3 Thereby the generalized Bessel function reduces to the ordinary Bessel function of the first kind. 
pump and probe pulses [32, 35, 46, 94, 131]. Additionally, LAPE was successfully utilized to study femto- to attosecond-dynamics [69, 132 137] and recently the first observation of LAPE from liquid surfaces has been made [138].

\subsection{High-harmonic generation}

High-harmonic generation was utilized in this thesis to generate EUV pulses, which were used to probe the out-of-equilibrium bandstructure of solids. In this section a very brief summary of the high-harmonic generation process should familiarize the unaccustomed reader with this EUV generation scheme. Much more details can be found in the literature and the interested reader is referred to review articles on the topic, e.g., [139, 140].

\section{Three-step model of high harmonic generation}

High harmonic generation can occur when an atom is subject to an intense laser pulse, which results in coherent EUV/XUV bursts and was first oberseved in 1987/88 [141, 142]. The three-step model of high-harmoinc generation, a semiclassical model to understand the microscopic processes behind the HHG, has been developed by Corkum and Kulander et al. [143, 144] and will be presented in this section (a full quantum mechanical description is also available, see, e.g., [145]). A schematic depiction of the three-step model is shown in figure 2.7. At first (1) the intense laser pulse disturbs the Coulomb potential of the atom, allowing for a tunnel ionization ${ }^{4}$ of an electron. Subsequently (2), the free electron is accelerated in the electric field of the laser pulse, while accumulating kinetic energy $E_{k i n}$. The oscillatory behavior of the electric field allows for trajectories of the electron returning to it's parent ion (see fig. 2.8) and therefore for a certain probability of the electron recombining with it's parent ion (3). The excess energy in this process, i.e., the ionization potential $I_{p}$ and the accumulated kinetic energy $E_{k i n}$ in the electric field, is set free in form of a photon.

Depending on the time of ionization $t_{i}$ the electron follows different trajectories, which can be calculated classically only taking into account the electric field of the laser pulse. In this calculation, moreover, the initial velocity $v$ and position $x$ of the electron upon ionization are assumed to coincide with the parent atom, i.e., $v\left(t_{i}\right)=0$ and $x\left(t_{i}\right)=0$ [150 152]. The result is shown in figure 2.8, where the y-axis shows the time-dependent position of the electron and the $\mathrm{x}$-axis describes the time in terms of the laser period $T$. The excess kinetic energy in the moment of recombination (i.e.,

4 Assuming a Keldysh parameter $\gamma=\sqrt{\frac{I_{p}}{2 U_{p}}} \ll 1$ [146], where tunnel ionization dominates. For $\gamma \approx 1$ the multiphoton ionization has to be taken into account additionally, which is usually relevant for short-wavelength driving laser pulses [147, 148]. For extreme laser parameters the Keldysh parameter is no good measure of the ionization process any more and additional care has to be taken [149]. 


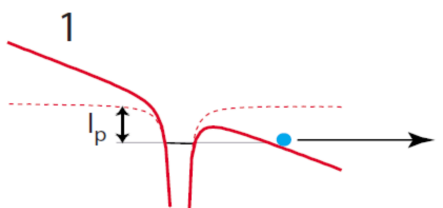

field/tunnel ionization

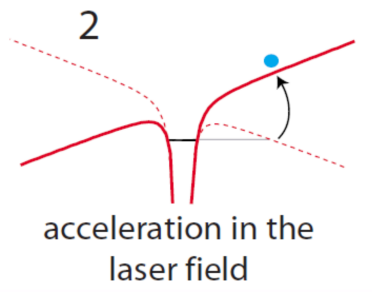

laser field

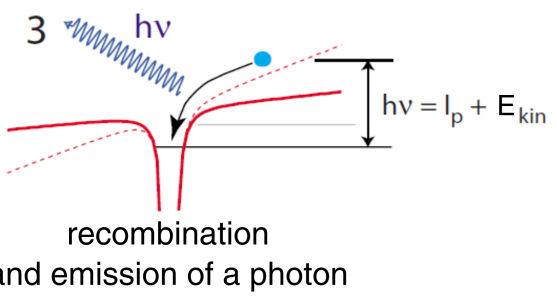

Figure 2.7.: Schematic depiction of the three step model. (1) The intense laser field disturbs the Coulomb potential so that an electron can tunnel into the continuum. (2) The electron is accelerated in the electric field, therby accumulating kinetic energy $E_{k i n}$, which is subsequently released (3) upon recombination in form of an EUV/XUV photon. Adapted with permission from Ref. [139]. Copyrighted by the American Physical Society.

when crossing $\mathrm{y}=0$ ) is colorcoded from red (lowest) to blue (highest). Moreover, one can notice that electrons which are ionized too early do no reencounter it's parent ion and thus they do not contribute to the HHG process. The maximum excess kinetic energy upon recombination can be calculated classically or quantum mechanically

to be $E_{\text {max }}=3.17 U_{P}$, with the ponderomotive potential $U_{P}=\frac{e^{2} E_{0}^{2}}{4 m_{e} w^{2}}$ describing the average kinetic energy of a free electron in an oscillatory electric field. Therefore, the cut-off energy is defined to be $E_{c u t-o f f}=I_{p}+3.17 U_{P}$ as the maximum photon energy reachable by the HHG process.

\section{The macroscopic high-harmonic spectrum}

In a reasonable description of the macroscopic high-harmonic spectrum, i.e., from several atoms and generated over several laser cycles, additional aspects have to be taken into account. At first, since the generation of high-harmonics occurs twice per laser cycle it results in a spectrum of harmonics spaced by two times the laser frequency $2 \omega$. This can easily be seen by considering the Fourier transform from the time- to the spectral domain. Additionally, only the odd harmonics make up the HHG spectrum, due to the inversion symmetry between upper- and lower half-cycles leading to destructive interference of the even orders.

Considering the macroscopic response of all gas atoms in the presence of the intense laser pulse the resulting EUV/XUV light from different atoms needs to add up coherently, i.e., the phase velocity of the driving laser has to match the phase velocity of the required harmonic. This is termed phase matching condition and can be expressed by the wave vector mismatch $(\Delta k=0)$ between the driver and the harmonic. In the HHG process the wave vector mismatch can be described by:

$$
\Delta k=\Delta k_{n}+\Delta k_{g}+\Delta k_{p}+\Delta k_{q}
$$




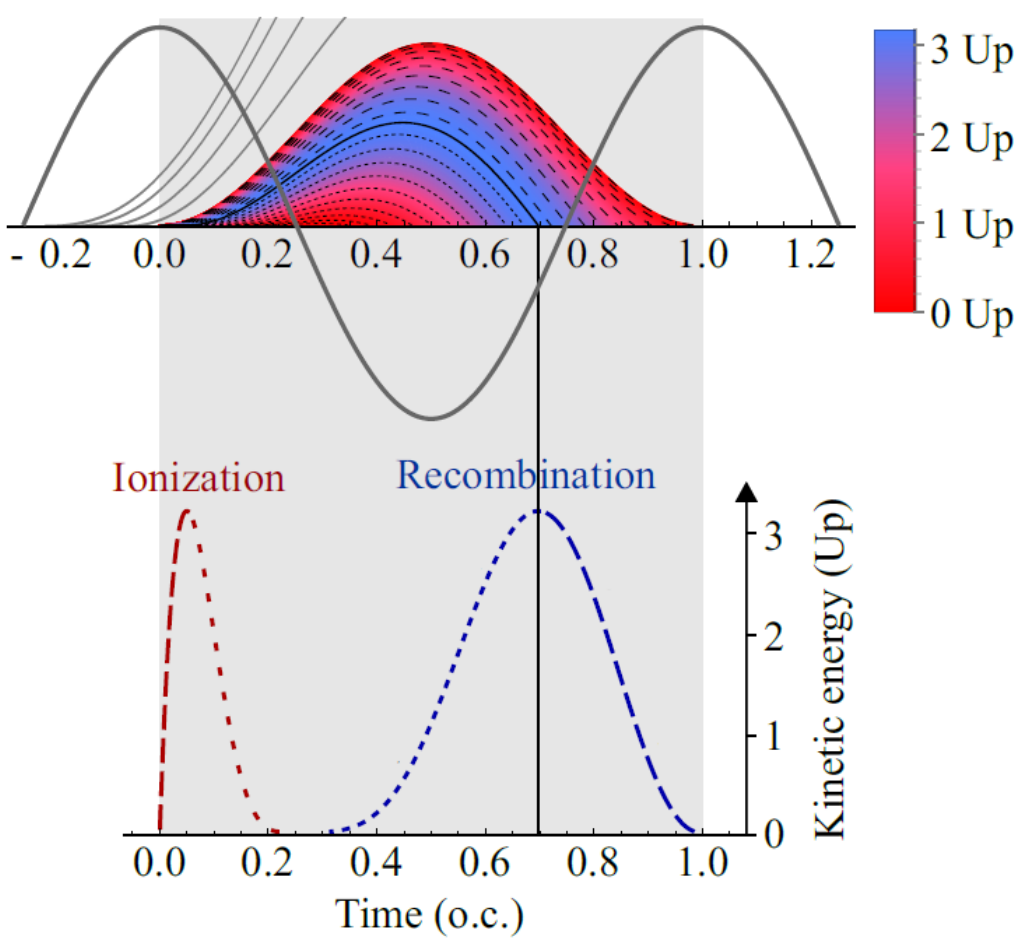

Figure 2.8.: Top: Classical trajectories of the electrons upon ionization at different times $t_{i}$ in comparison to the optical cycle of the laser pulse (solid grey line). The excess kinetic energy upon recombination with the parent ion is colorcoded in units of the ponderomotive potential $U_{p}$. Bottom: The time of ionization (red) and recombination (blue) and their kinetic energies showing the long- (long-dashed) and short-trajectories (shortdashed). Adjusted from [150].

with contributions from the dispersion of the neutral gas $\Delta k_{n}$ and the generated plasma $\Delta k_{p}$, the phase-mismatch due to the generation geometry $\Delta k_{g}$, and the phase-mismatch due to the single-atom dipole phase $\Delta k_{q}$, i.e., the phase the electron picks up while travelling in the continuum. A more detailed description of the different contributions can be found, e.g., in [139, 151-154]. Moreover, the reabsorption of the generated EUV/XUV light in the medium has to be considered [151].

\subsection{Static properites of graphene}

Graphene is the main topic in chapters 4 and 5 , where it was used to benchmark the newly built trMM setup and to study the role of band-crossing Auger recombination in it's dynamics after optical excitation. The static and dynamical properties of graphene will be briefly discussed here, with an emphasis on the dynamical properties, i.e., the optical excitation and subsequent relaxation of the electronic system. 


\subsubsection{Introduction}

Graphene is an allotrope of carbon, where the atoms form a two dimensional hexagonal lattice (see fig. 2.9), which has first been isolated (2004) and subsequently studied by the group of A. Geim. and K. Novoselov [155]. Both of them were awarded with the nobel prize in physics (2010) for their groundbreaking experiments. Graphene shows a manifold of interesting properties, e.g., the extraordinary mechanical strength [156, 157], the ultrahigh carrier mobility [158, 159] and the huge thermal conductivity [160, 161], which among others have sparked immense research and commercial interest.

\subsubsection{Structure}

The hexagonal lattice structure of graphene is described by a two-atom basis (A and $\mathrm{B}$ in figure 2.9 with lattice unit vectors $\mathbf{a}_{\mathbf{1}}=\frac{a}{2}(3, \sqrt{3})$ and $\mathbf{a}_{\mathbf{2}}=\frac{a}{2}(3,-\sqrt{3})$, where $a$ describes the carbon-carbon distance of $a \approx 1.42 \AA$ [162 164]. Each carbon atom is $s p^{2}$ hybridized, resulting in the trigonal planar structure of $\sigma$-bonds, which are responsible for the extraordinary mechanical strength [156]. These states are fully occupied and form a deep valence band. The residual electrons in the $p_{z}$ orbitals compose the delocalised $\pi$ - and $\pi^{*}$-bands, which are responsible for most of the striking electronic properties of graphene [165].
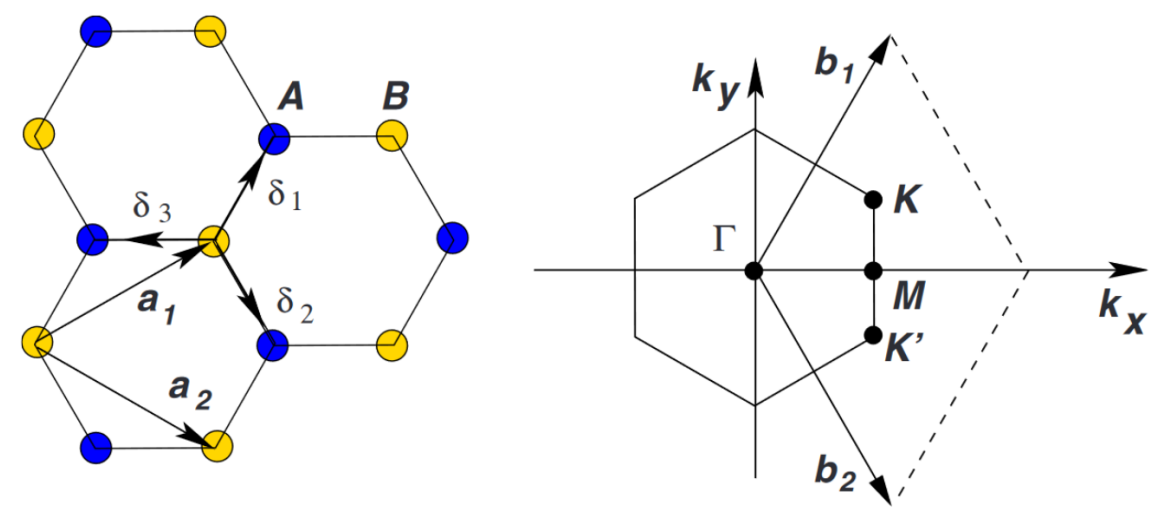

Figure 2.9.: Left: Real space structure of graphene, showing the two triangular sublattices A \& B (colored in blue and yellow) with the lattice unit vectors $a_{1}, a_{2}$. The vectors $\delta_{1}, \delta_{2}, \delta_{3}$ point towards the nearest-neighbours. Right: Brillouin zone of graphene with reciprocal lattice vectors $b_{1}, b_{2}$. Reprinted figure with permission from A. H. Castro Neto, et al., The electronic properties of graphene, Rev. Mod. Phys., 81:109-162, 2009, DOI:https://doi.org/10.1103/RevModPhys.81.109 [162]. (02021 by the American Physical Society. 


\subsubsection{Tight-binding description}

The tight-binding description for graphene, first done by P.R. Wallace [166] (1947), is quite successful in explaining the bandstructure and many of it's associated interesting properties. Since it will be the foundation for upcoming sections it will be briefly discussed here. The tight-binding Hamiltonian for graphene in secondquantization reads [162]:

$$
H=-t \sum_{\langle i, j\rangle, \sigma}\left(a_{\sigma, i}^{\dagger} b_{\sigma, j}+H . c .\right)-t^{\prime} \sum_{\langle\langle i, j\rangle\rangle, \sigma}\left(a_{\sigma, i}^{\dagger} a_{\sigma, j}+b_{\sigma, i}^{\dagger} b_{\sigma, j}+H . c .\right) \text {, }
$$

considering hopping between nearest and next-nearest neighbours with their respective energies $t$ and $t^{\prime}$. The operator $a_{\sigma, i}^{\dagger}\left(b_{\sigma, i}\right)$ creates (annihilates) an electron with spin $\sigma=(\uparrow, \downarrow)$ on site $R_{i}$ within sublattice A (B). The solutions to the single-particle Schrödinger equation have the form:

$$
E_{ \pm}\left(k_{x}, k_{y}\right)= \pm t \sqrt{3+f\left(k_{x}, k_{y}\right)}-t^{\prime} f\left(k_{x}, k_{y}\right)
$$

with $f\left(k_{x}, k_{y}\right)$ :

$$
f\left(k_{x}, k_{y}\right)=2 \cos \left(\sqrt{3} k_{y} a\right)+4 \cos \left(\frac{\sqrt{3}}{2} k_{y} a\right) \cos \left(\frac{3}{2} k_{x} a\right) .
$$

The eigenenergies $E_{ \pm}$as a function of the momenta $k_{x}, k_{y}$ describe the bandstructure, where - $(+)$ applies to the lower (upper) $\pi\left(\pi^{*}\right)$-band. The dispersion relation is shown in figure 2.10 for $t=2.7 \mathrm{eV}$ and $t^{\prime}=0.2 t$. The hopping energies can be inferred from first-principles calculations [167-169] or by comparision to experiments [170, 171]. The touching points between the $\pi$ - and $\pi^{*}$-band are located at $\mathrm{K}$ and K' points (see figure 2.9) and usually referred to as Dirac points due to their linear band disperion in their vicinity (see section 2.4.4).

\subsubsection{The 2D Dirac Hamiltonian of graphene}

All electronic excitations at low energies happen close the Dirac points in graphene. It is therefore sufficient to describe the bandstructure close to the $\mathrm{K}$ and $\mathrm{K}^{\prime}$ points in most of the cases. We start with the beforehand derived bandstructure of graphene in the tight-binding model. Expanding equation 2.27 around the $\mathrm{K}$ point for small momenta $\mathbf{q}$ (i.e., $\mathbf{k}=\mathbf{K}+\mathbf{q}$ ) up to second order in $\frac{\mathbf{q}}{\mathbf{K}}$ reads [162]:

$$
E_{ \pm}=3 t^{\prime} \pm \nu_{F}|\mathbf{q}|-\left(\frac{9 t^{\prime} a^{2}}{4} \pm \frac{3 t a^{2}}{8} \sin \left(3 \theta_{q}\right)\right)|\mathbf{q}|^{2}
$$

with the Fermi velocity $\nu_{F}=\frac{3 t a}{2} \approx 1 \cdot 10^{6} \frac{\mathrm{m}}{\mathrm{s}}$ and the angle $\theta_{q}=\arctan \left(\frac{q_{x}}{q_{y}}\right)$. The Fermi velocity is in first order independent of energy and momentum, which is a direct consequence of the linear dispersion relation. Moreover, a threefold symmetry 


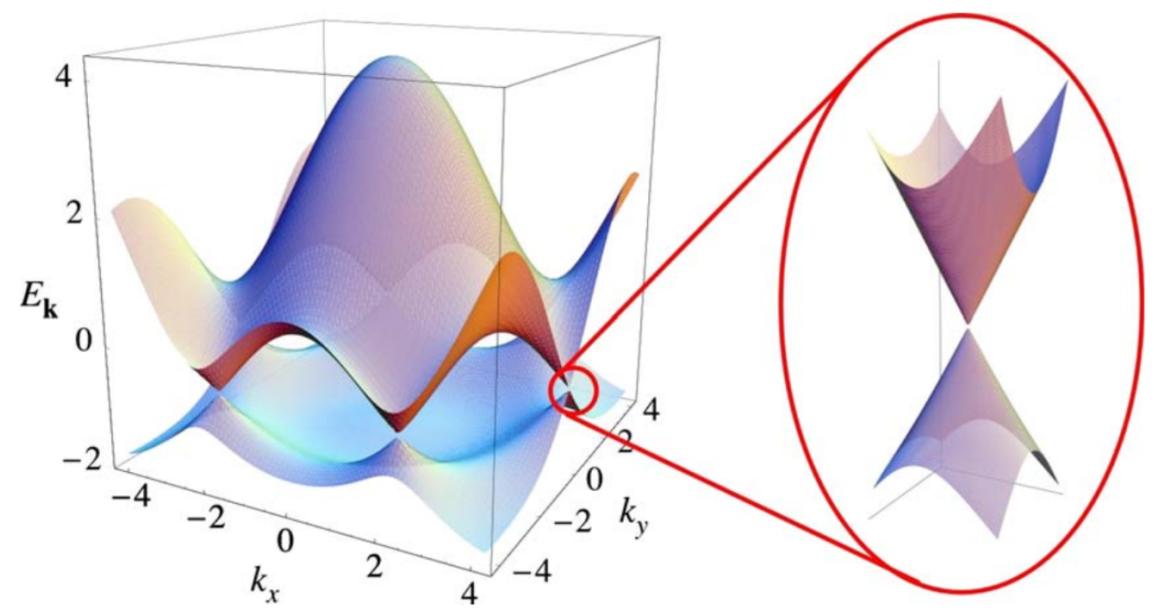

Figure 2.10.: Bandstructure $E\left(k=\left(k_{x}, k_{y}\right)\right)$ for graphene, which follows from the tight-binding Hamiltonian in equation 2.26. The hopping energies are chosen to $t=2.7 \mathrm{eV}$ and $t^{\prime}=-0.2 t$. The energy axis is in units of $t$. A close up near one of the Dirac points is shown, emphasizing the linear dispersion relation in it's vicinity. Reprinted figure with permission from A. H. Castro Neto, et al., The electronic properties of graphene, Rev. Mod. Phys., 81:109-162, 2009, DOI:https://doi.org/10.1103/RevModPhys.81.109 [162]. (O2021 by the American Physical Society.

$\left(\sin \left(3 \theta_{\mathbf{q}}\right)\right)$ in momentum space is obvious, which results in the so-called trigonal warping. This is shown in figure 2.11 .

Similarly, the Hamiltonian around the K-point can be expressed for $t^{\prime}=0$ as (for the derivation see, e.g., [162, 173]):

$$
H=\hbar \nu_{F} \mathbf{q} \cdot \sigma,
$$

with the Pauli matrices $\sigma=\left(\sigma_{x}, \sigma_{y}\right)$ resulting in the famous 2D Dirac equation in the vicinity of the $\mathrm{K}$ point:

$$
\hbar \nu_{F} \mathbf{q} \cdot \sigma \phi(\mathbf{q})=E \phi(\mathbf{q})
$$

with the two solutions:

$$
\phi_{\lambda= \pm 1}(\mathbf{q})=\frac{1}{\sqrt{2}}\left(\begin{array}{c}
1 \\
\pm e^{i \theta_{\mathbf{q}}}
\end{array}\right)
$$

for the corresponding eigenenergies $E=\lambda \hbar \nu_{F}= \pm \hbar \nu_{F}$ for the upper- and lower half of the Dirac cone. These eigenstates are also termed spinors (or spinor wave function) due to their similarity with spinor wavefunctions in Quantum electrodynamics (QED) [162, 174]. The derivation for the solutions and eigenenergies around the K' points follows the same routine and is therefore omitted here. 


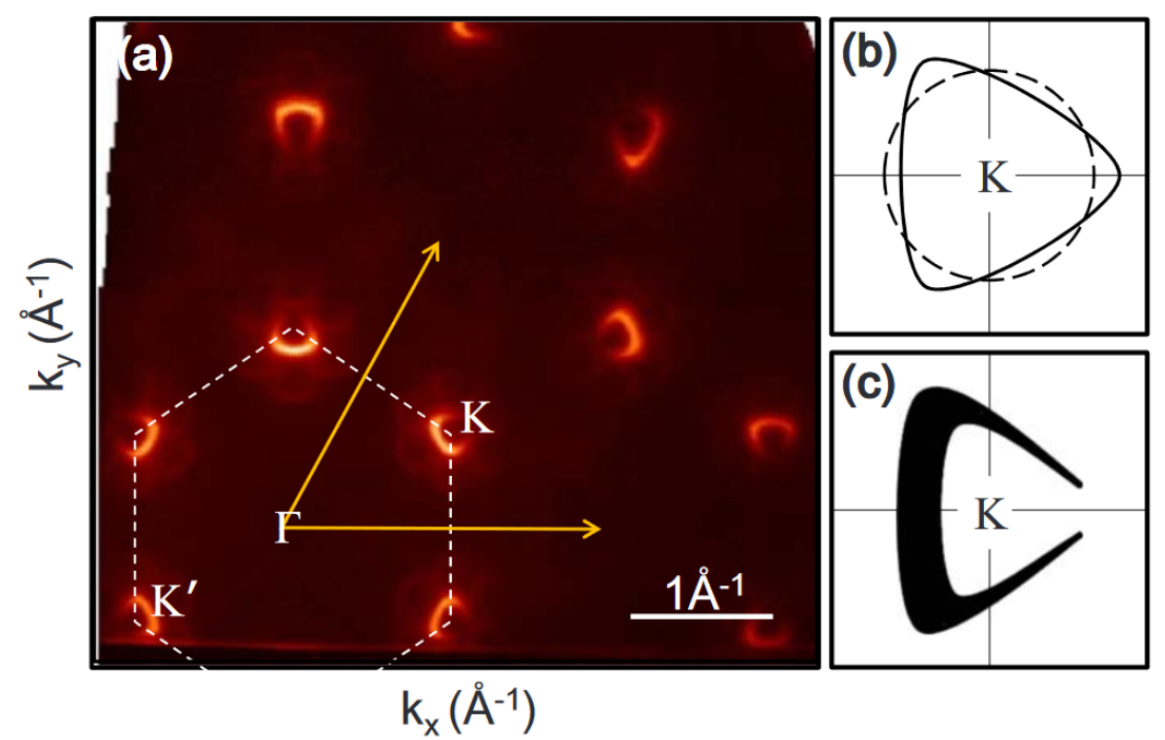

Figure 2.11.: (a) Fermi-surface map of graphene grown on SiC. The dotted line represents the boundary of the Brillouin zone. The arrows depict the reciprocal lattice vectors. (b) Fermi surface near the K point. The dashed line represents an ideal Dirac band, whereas the solid line shows the correction due to warping. (c) A schematic of the ARPES intensity, which is endcoded in the thickness of the line. The different intensity along the Dirac cone can be described with the dark corridor (see section 2.4.5). Reprinted figure with permission from C. Kim, et al., Imaging the electron density in solids by using multi-brillouin-zone angle resolved photoelectron spectroscopy, Phys. Rev. B, 82:235105, Dec 2010, DOI:https://doi.org/10.1103/PhysRevB.82.235105 [172]. (02021 by the American Physical Society.

\section{Pseudospin in graphene}

The eigenstates in graphene can be characterized according to their helicity, which is defined as [162]:

$$
\hat{h}=\frac{1}{2} \sigma \cdot \frac{\mathbf{p}}{|\mathbf{p}|},
$$

whereby the solutions in equation 2.32 are also eigenstates of $\hat{h}$ [162]:

$$
\hat{h} \phi_{\lambda}(\mathbf{q})=\frac{\lambda}{2} \phi_{\lambda}(\mathbf{q})
$$

which makes the helicity a good quantum number (within the 2D Dirac Hamiltonian). The pseudospin $\left(\frac{\lambda}{2}\right)$ thereby points either in $(\lambda=+1)$ or against $(\lambda=-1)$ the direction of momentum $\mathbf{q}$ (Note, that $\mathbf{q}$ is defined with respect to the $\mathrm{K}$ point and not the $\Gamma$ point). The concept of the pseudospin can be best understood within 
a Bloch sphere (see figure 2.12] [175, 178]. The pseudospin thereby points in the direction $(\sin (\phi) \cos (\theta)),(\sin (\phi) \sin (\theta)), \cos (\phi)$ which results in:

$$
|\Psi\rangle=\left(\begin{array}{c}
\cos (\phi / 2) \\
e^{i \theta} \sin (\phi / 2)
\end{array}\right)=\cos (\phi / 2) \Psi_{\mathrm{A}}(\mathbf{q})+e^{i \theta} \sin (\phi / 2) \Psi_{\mathrm{B}}(\mathbf{q})
$$

Here the state $|\Psi\rangle$ has been written out in the form of the two Bloch waves on the sublattices A and B (see, e.g., [162, 175-177] for the construction of the Bloch waves). A pseudospin pointing up (down) corresponds to a Bloch state having only sublattice $\Psi_{\mathrm{A}}\left(\Psi_{\mathrm{B}}\right)$ character, whereas the in-plane angle $\theta$ corresponds to the relative phase between the two Bloch waves on the sublattices A and B [175, 178]. Within the description of graphene by the 2D Dirac hamiltonian (eq. 2.30 the pseudospin only points in the equatorial plane (rotates only along $\theta$ ), i.e., both Bloch waves (on sublattices A and B) have always the same weight and only the respective phase changes. The pseudospin can aid in the description of several effects, i.e., the photoexcitation within the 2D Dirac Hamiltonian can be described as pseudospin-flip excitation [179] (see section 2.5.1) and Coloumb scattering can be seen as predominately pseudospin conserved (due to the coherence factor, see sec. 2.5.2.
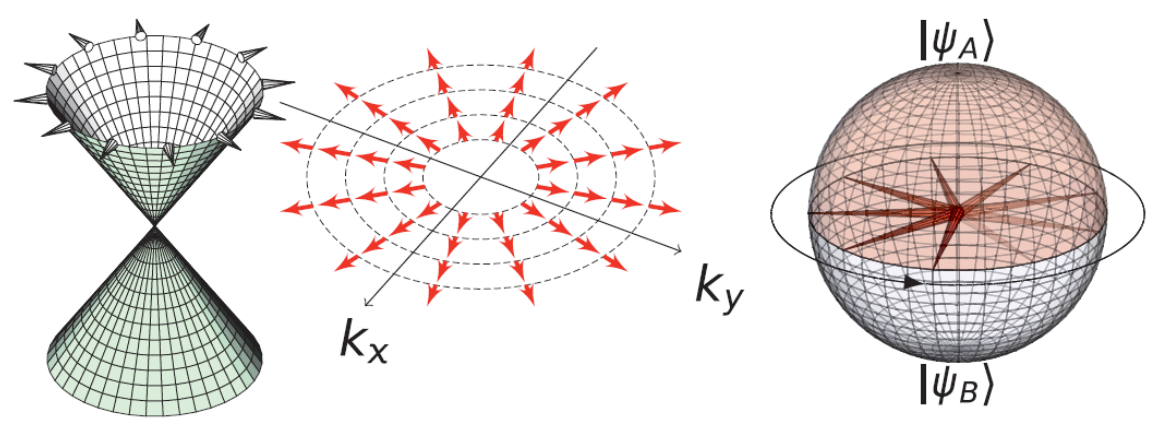

Figure 2.12.: Left: Bandstructure of graphene at the K-point with it's pseudospin orientation (depicted by the arrows). Middle: The pseudospin orientation is only dependent on the relative momentum and not the energy (sketched by the dashed circles). Right: The pseudospin representation on the Bloch sphere. Within the 2D Dirac Hamiltonian the pseudospin only rotates in the equatorial plane. This figure is taken from [178]. 


\subsubsection{Dark corridor in graphene}

The dark corridor in graphene describes the photoemission matrix element effect, which turns part of the Dirac cone invisible in photoemission (corresponding $\left|M_{f i, \lambda}\right|^{2} \approx$ 0 , see figure 2.11 and depends on the used photon energy and polarization of the probe beam [175, 180 183]. It can be understood within the three-step model with a final Bloch state expressed as [175, 181]:

$$
\left|\phi_{f}^{\text {Bloch }}\right\rangle=\frac{1}{\sqrt{2}}\left(\begin{array}{l}
1 \\
1
\end{array}\right)
$$

The photocurrent $I$ can then be expressed as:

$$
\begin{aligned}
I\left(E, \mathbf{q}_{\mathbf{f}}\right) \propto & \sum_{\lambda}\left|\left\langle\phi_{f}^{\text {Bloch }}\left(\mathbf{q}_{\mathbf{f}}\right)\left|H_{\text {int }}\right| \phi_{\lambda}\left(\mathbf{q}_{\mathbf{i}}\right)\right\rangle\right|^{2} \sum_{s}\left|\left\langle\Phi_{f, s}^{N-1} \mid \Phi_{i, \lambda}^{N-1}\right\rangle\right|^{2} \\
& \underbrace{\delta\left(E_{f}^{N}-E_{i}^{N}-\hbar \omega\right)}_{\text {energy conservation }} \underbrace{\delta\left(E-E_{f}^{N}+\phi\right)}_{\text {detection }},
\end{aligned}
$$

with the interaction Hamiltonian described by [184]:

$$
H_{\text {int }}=\frac{e v_{F}}{c}\left(\sigma_{x} A_{x}+\sigma_{y} A_{y}\right) .
$$

Seperating the spectral function into parts of the $\pi$ and $\pi^{*}$ band (corresponding to $\lambda= \pm 1$ ) the photocurrent is written as:

$$
\begin{aligned}
I\left(E, \mathbf{q}_{\mathbf{f}}\right) \propto & \sum_{\lambda} \underbrace{\left\langle\left.\left\langle\phi_{f}^{\text {Bloch }}\left(\mathbf{q}_{\mathbf{f}}\right)\left|H_{\text {int }}\right| \phi_{\lambda}\left(\mathbf{q}_{\mathbf{i}}\right)\right\rangle\right|^{2}\right.}_{\left|M_{f i, \lambda}\right|^{2}} A\left(\epsilon_{i}, \mathbf{q}_{\mathbf{i}}, \lambda\right) f\left(\epsilon_{i}\right) \\
& \underbrace{\delta\left(E_{f}^{N}-E_{i}^{N}-\hbar \omega\right)}_{\text {energy conservation }} \underbrace{\delta\left(E-E_{f}^{N}+\phi\right)}_{\text {detection }} .
\end{aligned}
$$

The matrix element $\left|M_{f i, \lambda}\right|^{2}$ results in:

$$
\begin{aligned}
\left|M_{f i, \lambda}\right|^{2} & =\left\langle\phi_{f}^{\text {Bloch }}\left(\mathbf{q}_{\mathbf{f}}\right)\left|H_{i n t}\right| \phi_{\lambda}\left(\mathbf{q}_{\mathbf{i}}\right)\right\rangle\left\langle\phi_{\lambda}\left(\mathbf{q}_{\mathbf{i}}\right)\left|H_{i n t}\right| \phi_{f}^{\text {Bloch }}\left(\mathbf{q}_{\mathbf{f}}\right)\right\rangle \\
\left|M_{f i, \lambda}\right|^{2} & =\frac{e^{2} v_{f}^{2} \mathbf{A}^{2}}{c^{2}} \frac{1}{4}\left(\begin{array}{ll}
e^{i \theta_{\mathbf{A}}} & e^{-i \theta_{\mathbf{A}}}
\end{array}\right)\left(\begin{array}{cc}
1 & \lambda e^{-i \theta_{\mathbf{q}}} \\
\lambda e^{i \theta_{\mathbf{q}}} & 1
\end{array}\right)\left(\begin{array}{c}
e^{-i \theta_{\mathbf{A}}} \\
e^{i \theta_{\mathbf{A}}}
\end{array}\right) \\
\left|M_{f i, \lambda}\right|^{2} & =\frac{e^{2} v_{f}^{2} \mathbf{A}^{2}}{c^{2}} \frac{1}{2}\left(1+\lambda \cos \left(\theta_{\mathbf{q}}-2 \theta_{\mathbf{A}}\right)\right) .
\end{aligned}
$$

The approximation of a final Bloch state seems to be justified for the used photon energy of $26.5 \mathrm{eV}$ in this thesis. Only for higher photon energies $\hbar \omega>40 \mathrm{eV}$ deviations were observed, as has been nicely shown by Gierz et al. [180], where they were able to illuminate the dark corridor for $\hbar \omega=52 \mathrm{eV}$ (s-pol.). This behavior was understood by taking into account time-reversed spin-polarized low-energy electron 
diffraction (SPLEED) states for the final state in photoemission.

\subsection{Dynamical properties of graphene}

Whenever an electronic system is brought out-of-equilibrium by, e.g., an optical excitation, the overall dynamics are governend by the different interactions present. These many-body interactions play also an important role in the following dynamics of graphene. From previous experimental- as well as theoretical studies a description of the hot charge carrier dynamics in graphene has evolved (see figure 2.13). At first, the optical excitation in graphene creates an asymmetric distribution of electrons and holes in the Dirac cone, which is described by an angle dependent transition probability [179, 184-191]. After that a hot Fermi-Dirac distribution is established within the electron $\left(\mathrm{e}^{-}\right)$and the hole $\left(\mathrm{h}^{+}\right)$system on several tens of femtoseconds by Coulomb scattering, however with momentum-dependent temperatures [4, 36, 179, 192-200. Subsequently, the isotropisation, thermalization in-between the $\mathrm{e}^{-}$- and $\mathrm{h}^{+}$-system, and cooling of the electronic system is dominated by scattering with optical phonons on a timescale of $\sim 200$ fs [185, 189, 201 206]. Finally, cooling via acoustic phonons/supercollisions brings the system back to equilibrium on ps timescales [201, 205, 207 214]. Obviously, the overall dynamics and timescales depend on several conditions (doping, screening, pump fluence, ...) and a clear separation between all the temporally overlapping processes is not always possible.

In the following part the different steps describing the hot charge carrier dynamics in graphene will be discussed in more detail.

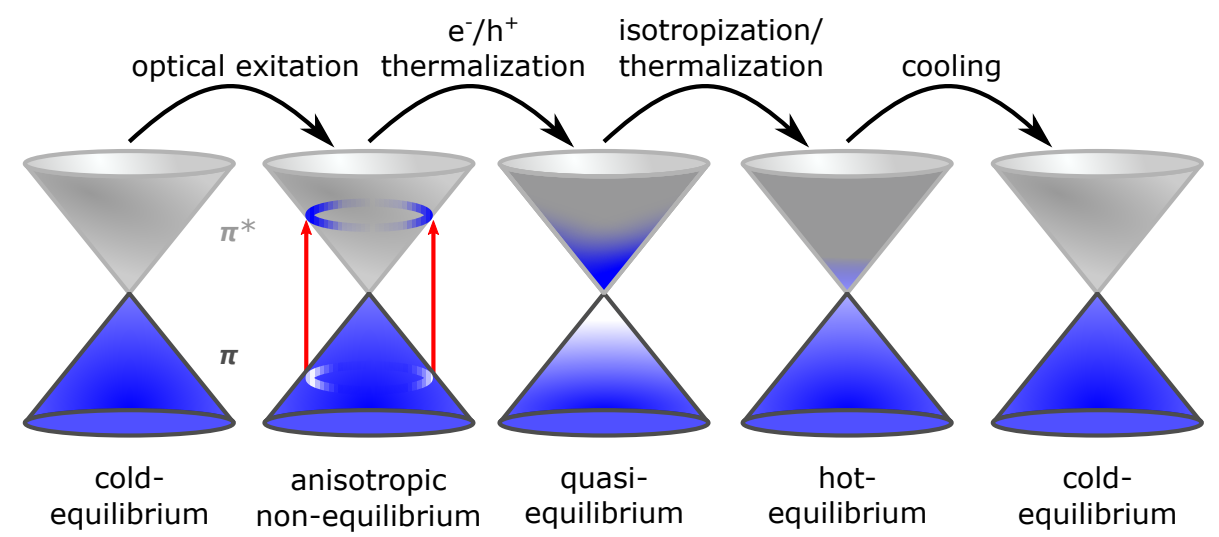

Figure 2.13.: Dynamics in graphene after an optical excitation. The excitation by light leads to an anisotropic carrier distribution which thermalizes on several tens of fs towards a quasi-equilibrium with still a momentum dependent temperature. Further isotropization and thermalization leads to a homogeneous hot-equilibrium (i.e., described by a single FermiDirac distribution), which cools back towards the cold-equilibrium on ps timescales. After [175]. 


\subsubsection{Optical excitation}

The anisotropic distribution after an optical excitation has been predicted for graphite [186] and subsequently for graphene [184, 185] and was experimentally observed by several groups [179, 187 191, 215]. It can be easily understood by taking into account the two-dimensional Dirac Hamiltonian and Fermi's golden rule. The transition rate $\omega_{f i}$ from the initial state to the final state then reads:

$$
\omega_{f i}=\frac{2 \pi}{\hbar}\left|\left\langle\phi_{f}\left(\mathbf{q}_{f}\right)\left|H_{i n t}\right| \phi_{i}\left(\mathbf{q}_{i}\right)\right\rangle\right|^{2} \delta\left(E_{f}-E_{i}-\hbar \omega\right),
$$

with $\phi_{f}, \phi_{i}$ being the final- and initial state wavefunction and $H_{\text {int }}$ the interaction Hamiltonian, while $\delta\left(E_{f}-E_{i}-\hbar \omega\right)$ ensures energy conservation. The momentum transfer from the photon can be neglected since $\mathbf{k}_{\text {photon }} \ll \mathbf{q}_{i}, \mathbf{q}_{f}$, resulting in a vertical transition: $\mathbf{q}_{i}=\mathbf{q}_{f}=\mathbf{q}$. The interaction Hamiltonian has already been introduced in sec. 2.4.5. The transition rate $\omega_{f i}$ is then given by (using the solutions from eq. 2.32):

$$
w_{f i}=\frac{2 \pi}{\hbar}\left|\frac{e v_{F}}{c}\left(-i A_{x} \sin \left(\theta_{\mathbf{q}}\right)+i A_{y} \cos \left(\theta_{\mathbf{q}}\right)\right)\right|^{2} .
$$

Using polar coordinates for the vector potential, i.e., $A_{x}=A \cos \left(\theta_{\mathbf{A}}\right)$ and $A_{y}=$ $A \sin \left(\theta_{\mathbf{A}}\right)$, the transition rate becomes:

$$
w_{f i}=\frac{2 \pi}{\hbar} \frac{e^{2} v_{F}^{2} \mathbf{A}^{2}}{c^{2}} \sin ^{2}\left(\theta_{\mathbf{A}}-\theta_{\mathbf{q}}\right) .
$$

From here the anisotropic excitation can directly be seen. It is maximal in the direction perpendicular to the vector potential (or electric field), whereas there is no excitation in the direction parallel to it as shown in figure 2.14 [175, 188, 216]. Depending on the scattering dynamics the optical excitation can saturate due to an increased state blocking (Pauli blocking) which becomes increasingly important for fluences in the $\mathrm{mJ} / \mathrm{cm}^{2}$-range [216].
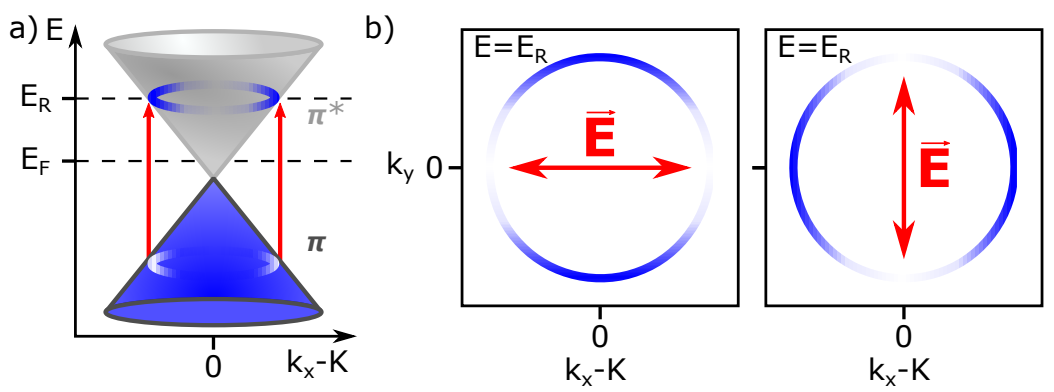

Figure 2.14.: a) The optical excitation (red arrows) leads to anisotropic distribution of carriers (shaded blue) where the resonance condition is fulfilled ( $E=$ $\left.E_{R}\right)$. b) Optical interband transition rate (shaded blue) for different directions of the electric field vector (red arrow). After [175]. 


\subsubsection{Electron-electron scattering}

The Coulomb mediated scattering processes dominate the dynamics in graphene for the first $0-50$ fs and their interplay determine effects like, i.e., the carrier multiplication [4, 192 194] and the population inversion [36, 194, 195], both of which have sparked immense research interest due to the possible application in optoelectronics [217]. While in general the Coulomb interaction is long range it is subject to screening by mobile charge carriers in solid state physics, leading to an effective short range interaction. In graphene, however, screening can be substantially reduced by it's dimensionality and the vanishing density of states near the Dirac point [218, 219].

In this section, the main aspects of Coulomb mediated scattering mechanisms in graphene will be discussed briefly within the linear dispersing bands (see, e.g., [220222 for a discussion about additional effects, like the trigonal warping or band renormalization on the scattering). In figure 2.15 the different two body Coulomb mediated scattering mechanisms for an n-doped graphene sample are sketched. The scattering events can occur in the same band as intraband scattering or between different bands as interband scattering, whereby the Auger-like processes increase (Impact excitation, short: IE) or decrease (Auger Recombination, short: AR) the number of carriers in the conduction band.

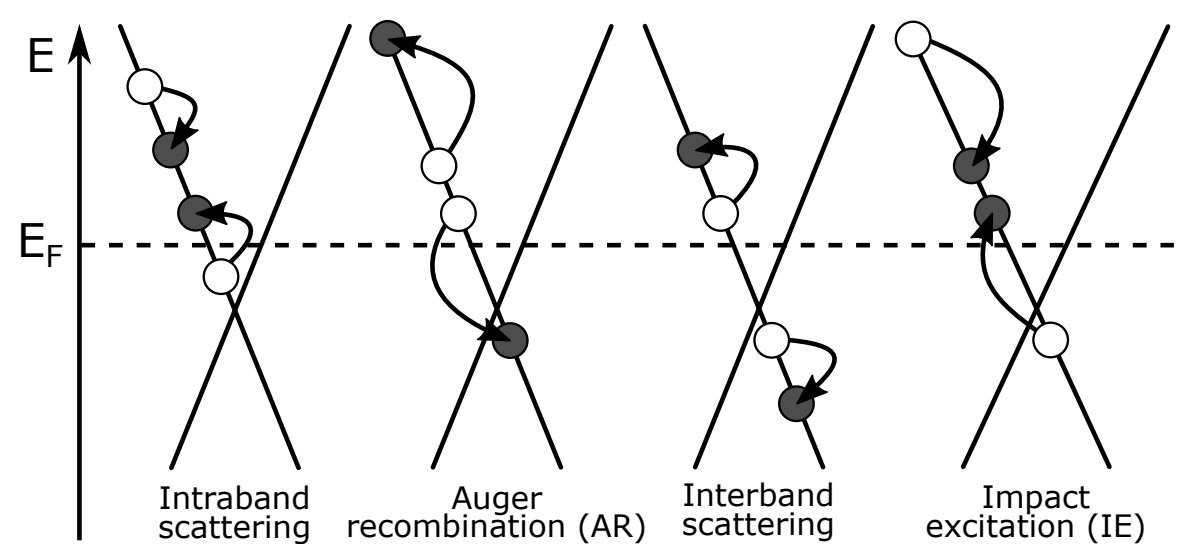

Figure 2.15.: Sketch of two body Coulomb-mediated scattering events in photoexcited n-doped graphene. The Fermi edge is denoted by the dotted line and the arrows mark the transition from the initial state to the final state in the scattering process. The scattering events can occur in the same band as intraband scattering and between bands as interband scattering. The Auger-like processes increase (Impact excitation; IE) or decrease (Auger recombination; AR) the number of carriers in the conduction band.

The transition rate per unit time for an electron with momentum $\mathbf{k}_{\mathbf{1}}$ scattering 
to a momentum $\mathbf{k}_{\mathbf{1}}^{\prime}$ can be expressed by Fermi's golden rule as [223]:

$$
\begin{aligned}
w_{\mathbf{k}_{\mathbf{1}}^{\prime}, \mathbf{k}_{\mathbf{1}}}= & \frac{2 \pi}{\hbar} \sum_{\mathbf{q}, \mathbf{k}_{\mathbf{2}}, \mathbf{k}_{\mathbf{2}}^{\prime}} f_{\mathbf{k}_{\mathbf{2}}}\left(1-f_{\mathbf{k}_{\mathbf{2}}^{\prime}}\right) F_{\mathbf{k}_{\mathbf{2}}, \mathbf{k}_{\mathbf{2}}^{\prime}}\left|\tilde{V}_{\mathbf{q}}\right| \\
& \underbrace{\delta\left(\mathbf{k}_{\mathbf{1}}+\mathbf{k}_{\mathbf{1}}^{\prime}+\mathbf{q}\right) \delta\left(\mathbf{k}_{\mathbf{2}}^{\prime}-\mathbf{k}_{\mathbf{2}}-\mathbf{q}\right)}_{\text {momentum conservation }} \underbrace{\delta\left(\epsilon_{1}+\epsilon_{2}-\epsilon_{1}^{\prime}-\epsilon_{2}^{\prime}\right)}_{\text {energy conservation }},
\end{aligned}
$$

with the momentum transfer $\mathbf{q}$, the occupation function $f_{\mathbf{k}_{\mathbf{2}}}$, the coherence factor $F_{\mathbf{k}_{\mathbf{2}}, \mathbf{k}_{\mathbf{2}}^{\prime}}$ (sometimes also referred to as chirality- or Dirac factor) and the screened Coulomb potential $\tilde{V}_{\mathbf{q}}$ given as [185, 223, 224]:

$$
\tilde{V}_{\mathbf{q}}=\frac{V_{\mathbf{q}}}{\tilde{\epsilon}(\mathbf{q})} ; \quad V_{\mathbf{q}}=\frac{2 \pi e^{2}}{|\mathbf{q}| \epsilon_{e f f}}
$$

Whereby, $\epsilon_{\text {eff }}$ describes the effective static dielectric constant (due to the substrate environment) and $\tilde{\epsilon}(\mathbf{q})$ describes the corrections due to screening, which can be included statically [185, 225] or dynamically [223, 226 228].

The coherence factor $F_{\mathbf{k}_{2}, \mathbf{k}_{\mathbf{2}}^{\prime}}$ arises due to projection of initial onto final spinor states and results in [207, 221, 229]:

$$
F_{\mathbf{k}_{\mathbf{2}}, \mathbf{k}_{\mathbf{2}}^{\prime}}=\frac{1}{2}\left(1+\lambda_{\mathbf{k}_{\mathbf{2}}} \lambda_{\mathbf{k}_{\mathbf{2}}^{\prime}} \cos \left(\theta_{\mathbf{k}_{\mathbf{2}}}-\theta_{\mathbf{k}_{\mathbf{2}}^{\prime}}\right)\right)
$$

with $\lambda=1(-1)$ for the upper (lower) half of the Dirac cone, as derived in section 2.4.4. From this point of view already some major statements can be made about Coulomb scattering in graphene:

i The dielectric environment rescales the coulomb interaction and therefore directly influences the observed dynamics in graphene [230].

ii The intervalley ( $\mathrm{K}$ to $\mathrm{K}^{\prime}$ ) scattering is suppressed with increasing momentum transfer $\mathbf{q}$ and is therefore neglected in the following discussion [185, 231, 232].

iii The electron-electron dynamics show a strong fluency [189, 193, 216, 233, 234], pump photon energy [189, 233], and doping dependence [235], which can be understood due to the difference in available phase space (due to the occupation functions $f_{\mathbf{k}}$ ) for the respective scattering pathways. In general with increasing fluency faster dynamics can be expected [189, 193, 216, 233, 234], whereby even Pauli blocking [195, 197] can occur and influence the dynamics.

\section{Intraband scattering}

Intraband scattering is a process where $\mathbf{k}_{\mathbf{1}}, \mathbf{k}_{\mathbf{1}}^{\prime}, \mathbf{k}_{\mathbf{2}}, \mathbf{k}_{\mathbf{2}}^{\prime}$ are within the same band $\left(\lambda_{\mathbf{k}_{\mathbf{1}}}=\lambda_{\mathbf{k}_{\mathbf{1}}^{\prime}}=\lambda_{\mathbf{k}_{\mathbf{2}}}=\lambda_{\mathbf{k}_{\mathbf{2}}^{\prime}}\right)$. From the coherence factor we can see that:

$$
F_{\mathbf{k}_{\mathbf{2}}, \mathbf{k}_{\mathbf{2}}^{\prime}}=\frac{1}{2}\left(1+\cos \left(\theta_{\mathbf{k}_{\mathbf{2}}}-\theta_{\mathbf{k}_{\mathbf{2}}^{\prime}}\right)\right)
$$


thus scattering is pronounced in the collinear direction $\left(\theta_{\mathbf{k}_{\mathbf{2}}}=\theta_{\mathbf{k}_{\mathbf{2}}^{\prime}}\right)$, whereas backscattering is forbidden $\left(\cos \left(\Delta \theta_{\mathbf{k}_{\mathbf{2}}, \mathbf{k}_{\mathbf{2}}^{\prime}}=\pi\right)=-1\right)$ [185, 189, 204].

\section{Interband scattering}

The interband scattering describes a scattering event between the two bands, where the number of carriers in each band stays the same, in contrast to Auger scattering. Also here, the coherence factor leads to predominately collinear scattering, take for example $\mathbf{k}_{\mathbf{1}}, \mathbf{k}_{\mathbf{2}}^{\prime}$ in the $\pi$-band $\left(\lambda_{\mathbf{k}_{\mathbf{1}}}=\lambda_{\mathbf{k}_{\mathbf{2}}^{\prime}}=1\right)$ and $\mathbf{k}_{\mathbf{1}}^{\prime}, \mathbf{k}_{\mathbf{2}}$ in the $\pi^{*}$-band $\left(\lambda_{\mathbf{k}_{\mathbf{1}}}=\right.$ $\left.\lambda_{\mathbf{k}_{\mathbf{2}}^{\prime}}=-1\right)$. The coherence factor then reads:

$$
F_{\mathbf{k}_{\mathbf{2}}, \mathbf{k}_{\mathbf{2}}^{\prime}}=\frac{1}{2}\left(1-\cos \left(\theta_{\mathbf{k}_{\mathbf{2}}}-\theta_{\mathbf{k}_{\mathbf{2}}^{\prime}}\right)\right)
$$

which results in $\cos \left(\Delta \theta_{\mathbf{k}_{2}, \mathbf{k}_{\mathbf{2}}^{\prime}}=\pi\right)=-1$ for a maximal transition rate. Note that now a scattering process is described, where the Dirac point was crossed, resulting in a collinear scattering with $\Delta \theta_{\mathbf{k}_{\mathbf{2}}, \mathbf{k}_{\mathbf{2}}^{\prime}}=\pi$ across the two bands.

\section{Auger processes}

The Auger processes impact excitation (IE) and Auger recombination (AR) are the only two-body coulomb mediated scattering processes that change the number of carriers in between the two bands [232]. Let us assume an impact excitation (IE) process with $\mathbf{k}_{\mathbf{1}}, \mathbf{k}_{\mathbf{1}}^{\prime}, \mathbf{k}_{\mathbf{2}}^{\prime}$ in the $\pi^{*}$-band and $\mathbf{k}_{\mathbf{2}}$ in the $\pi$-band. From energy- and momentum conservation within the linear dispersing bands we get:

$$
\begin{aligned}
\hbar v_{F}\left|\mathbf{k}_{\mathbf{1}}\right|-\hbar v_{F}\left|\mathbf{k}_{\mathbf{2}}\right| & =\hbar v_{F}\left|\mathbf{k}_{\mathbf{1}}^{\prime}\right|+\hbar v_{F}\left|\mathbf{k}_{\mathbf{2}}^{\prime}\right| \\
\mathbf{k}_{\mathbf{1}}+\mathbf{k}_{\mathbf{2}} & =\mathbf{k}_{\mathbf{1}}^{\prime}+\mathbf{k}_{\mathbf{2}}^{\prime},
\end{aligned}
$$

which can only be fulfilled at the same time if $\mathbf{k}_{\mathbf{1}}, \mathbf{k}_{\mathbf{1}}^{\prime}, \mathbf{k}_{\mathbf{2}}, \mathbf{k}_{\mathbf{2}}^{\prime}$ lie on the same line [196, 229]. Ergo, Auger processes within the linear dispersing bands are strictly collinear. IE and AR affect the energetic spectrum of the carriers differently, which will be used in chapter 5 to distinguish the different processes. This is shown in figure 2.16, where the carrier spectrum is plotted for three different scattering pathways (AR, IE and intraband scattering). Intraband scattering leads to a symmetric smearing of the initially excited carrier distribution, whereas IE (AR) leads to a shift to lower (higher) energies.

The interplay between Auger recombination and impact excitation influences the carrier multiplication on short time scales (0 - $50 \mathrm{fs})$ and is obviously doping and fluency dependent, whereby in general n-doping and higher fluencies favour Auger recombination [235, 236]. A first direct evidence of impact excitation by tr-ARPES has been found by Gierz et al. in p-doped graphene [200]. For Auger recombination the first direct evidence is still missing, which was the motivation for the study conducted in chapter 5 . 

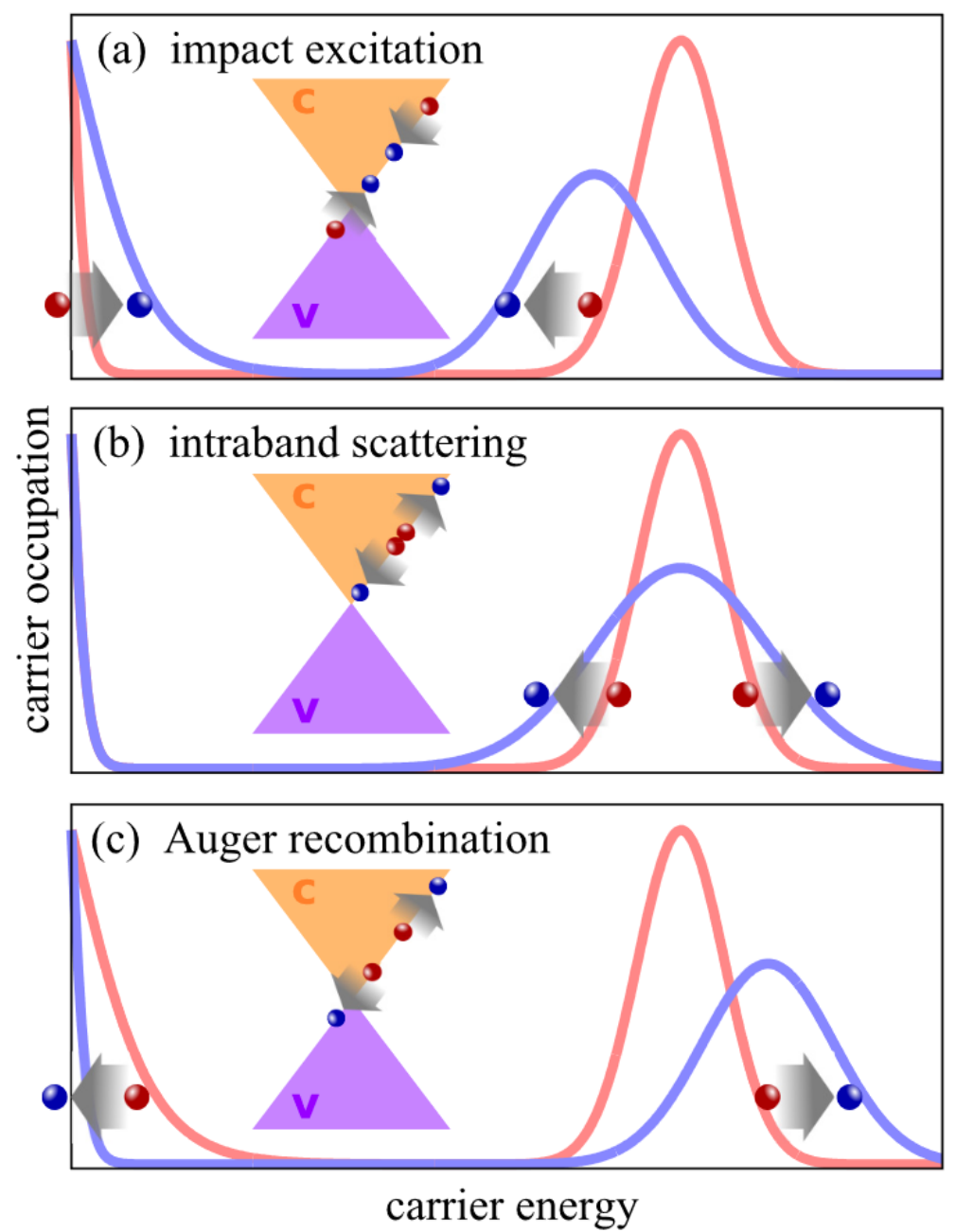

Figure 2.16.: Two-body Coulomb mediated scattering processes and their influence on the carrier spectrum. (a) Impact excitation (IE) accumulates carriers at lower energies and redshifts the optically excited occupation, (b) intraband scattering leads to a spectral broadening around the excitation energy, and (c) Auger recombination (AR) leads to a blueshift of the excited carriers. Reprinted figure with permission from E. Malic, et al., Experimentally accessible signatures of auger scattering in graphene, Phys. Rev. B, 94(23):235430, Dec 2016, DOI:https://doi.org/10.1103/PhysRevB.94.235430 [236]. (O2021 by the American Physical Society.

\subsubsection{Electron-phonon scattering \& supercollisions}

The scattering with optical phonons influences the ultrafast (50 - $200 \mathrm{fs}$ ) thermalization and isotropization of the electron and hole distributions, as well as the overall cooling of the electronic system on timescales of $0.5-2$ ps [185, 189, 201-206], 
whereby mostly the twofold-degenerat ${ }^{5} E_{2 g}$ mode with 48 Thz $(\approx 200 \mathrm{meV})$ at the $\Gamma$-point and the $A_{1}^{\prime}$ mode at the K-point with $39 \mathrm{THz}(\approx 160 \mathrm{meV})$ participate (see figure 2.17] [201, 208, 237, 238]. This strong coupling can lead to a steplike relaxation of the carrier distribution (in steps of $\hbar \omega$ ) [185], which has already been seen in graphite by trARPES as so-called phonon-induced replica (PIR) [239].

The overall cooling slows down when the carrier and optical phonon temperatures become nearly identical (also termed hot optical phonon bottleneck) and further decelerates when the carriers do not have enough energy to emit an optical phonon (optical phonon energy bottleneck) [201, 205, 207 210]. Subsequently, the system cools down via the anharmonic decay of optical phonons into acoustic ones [201, 211], direct emission of acoustic phonons (constrained by the small Fermi surface and momentum conservation) [207, 208, 212] and disorder-assisted electron-phonon scattering (also termed supercollisions) [207, 213, 214]. The cooling of graphene and graphite and the role of the different relaxation pathways has been studied by many groups from theoretical and experimental side and is still an ongoing research area.

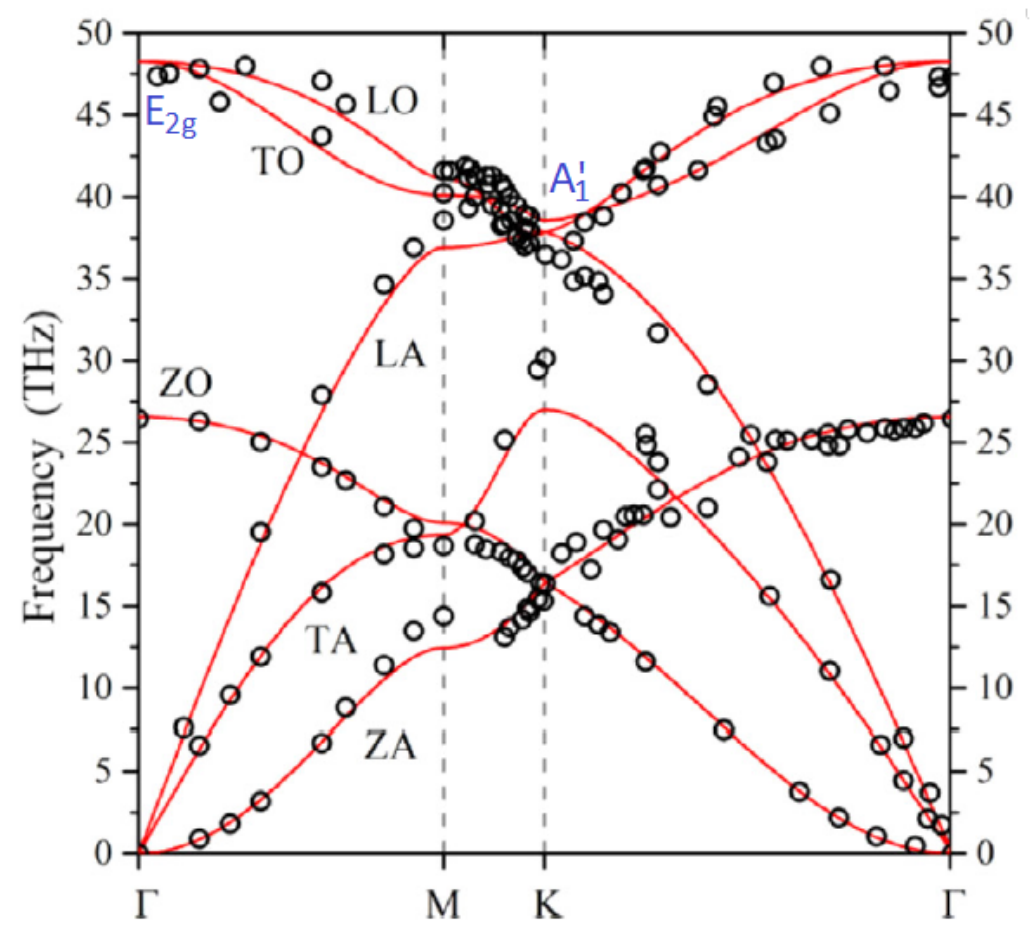

Figure 2.17.: Calculated phonon dispersion curve for Graphene (red lines) and experimental data (open circles, from [237, 240]). Mostly the $E_{2 g}$ and the $A_{1}^{\prime}$ mode participate in the relaxation of the electronic system. Adapted from [241].

5 The $E_{2 g}$ mode splits into the in-plane optical mode (TO) and longitudinal optical mode (LO). 


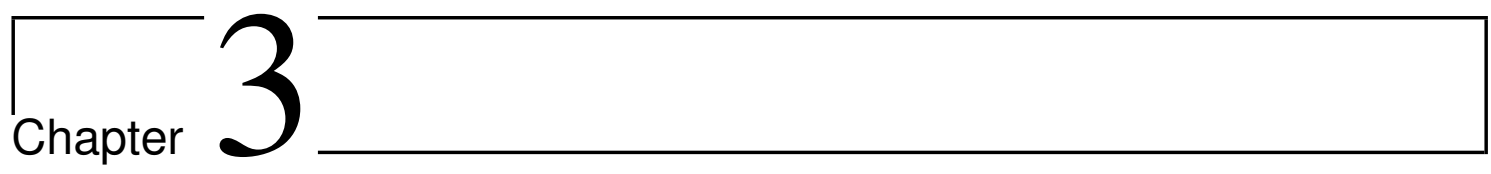

\section{Experimental setup}

The main topic of this thesis was the successful build-up of the $1 \mathrm{MHz}$ time-resolved momentum microscopy (trMM) setup, which will be explained in the subsequent sections. Apart from benchmarking of the system (chapter 4) first results on scattering dynamics in graphene (chapter 5), light-dressed bandstructures (chapter 6) and orbital imaging [77] could be gained. Parts in this chapter are reprinted from the publication: M.Keunecke, et al.:"Time-Resolved Momentum Microscopy with a $1 \mathrm{MHz}$ High-Harmonic Extreme Ultraviolet Beamline", Review of Scientific Instruments, 91(6):063905, 2020 [78] with the permission of AIP Publishing. The author of this thesis contributed as follows: The author had a significant contribution in planning and building up the trMM setup (except for the high-harmonic generation, which was planned and build up by Christina Möller, Marie Gutberlet and Amelie Schulte). He performed the corresponding measurements, data analysis, and interpretation mainly together with David Schmitt, and took part in writing of the manuscript.

\subsection{The femtosecond $1 \mathrm{MHz}$ EUV beamline}

We start our discussion with the light source, which has to fulfill several key requirements with respect to the photon energy, intensity, repetition rate, pulse width and spectral bandwidth. The photon energy should be at least in the EUV regime in order to access the full Brillouin zone of the electronic band structure (see equation 2.13). For a maximal detection rate of a time-of-flight detector, like it was used throughout this thesis, with minimal distortions due to space-charge effects, a $\mathrm{MHz}$ repetition rate light source with a moderate number of photons per pulse is desirable. Also, time-resolution and spectral bandwidth, which are connected via the time-bandwidth product, need to be adapted to the envisaged experiments. The light source should also provide synchronized pump pulses with tunable wavelength, pulse duration and intensity. In this section, such a beamline based on a table-top, high repetition-rate fiber amplifier laser is described. A schematic layout of the 
system is shown in figure 3.1 .

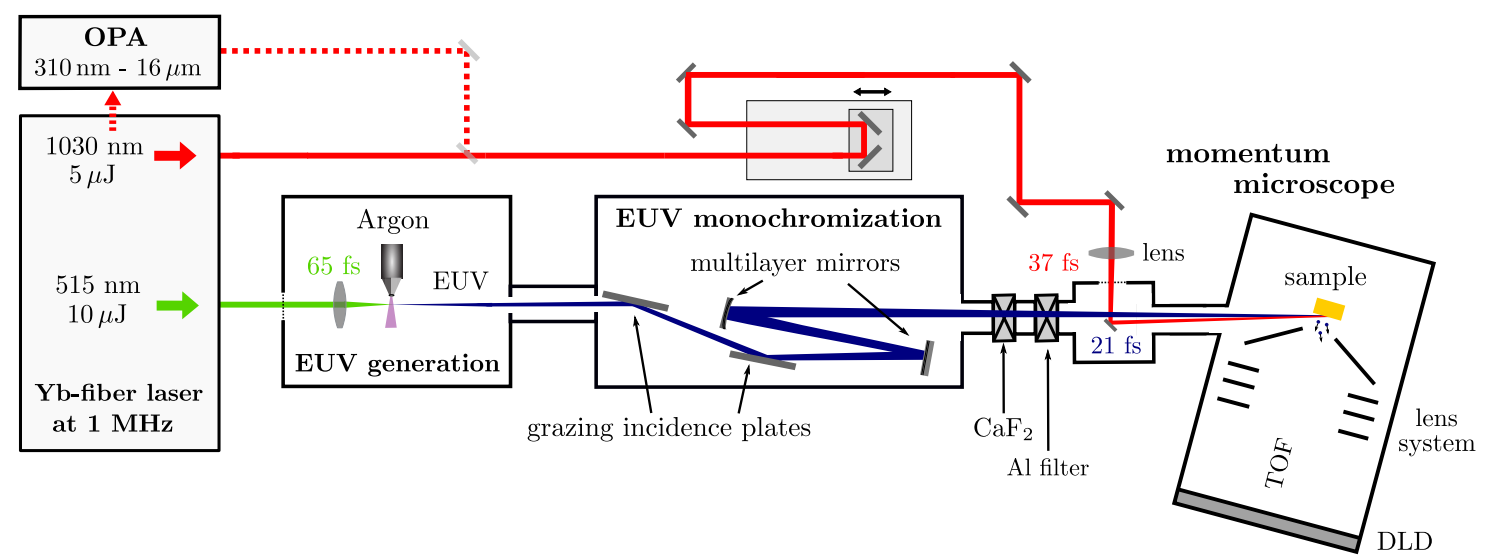

Figure 3.1.: Schematic layout of the experimental setup consisting of the $1 \mathrm{MHz}$ EUV beamline, the pump line and the momentum microscope. A detailed description is given in the respective sections. Taken from [78].

\subsubsection{High repetition rate fiber amplifier system}

Throughout this thesis an ytterbium (Yb)-doped fiber amplifier (Active Fiber Systems) which delivers $100 \mathrm{\mu J}$ pulses at a repetition rate of $1 \mathrm{MHz}$ centered around $1030 \mathrm{~nm}$ with a pulse length of approximately 250-300 fs (Gaussian) was used. These pulses are spectrally broadened through self-phase modulation in a $1 \mathrm{~m}$ hollow-core fiber (HCF) filled with 12 bar krypton gas and compressed with a pair of chirped mirrors resulting in a pulse duration of $\sim 35-40$ fs (Gaussian, central pulse) at $50 \mu \mathrm{J}$ pulse energy [242, 243]. An exemplary spectrum and an intensity autocorrelation (AC) measurement of the compressed pulses can be found in figure 3.2. Note that the autocorrelation measurement cannot be well described by a Gaussian pulse shape due to an additional post-pulse which is delivered by the fiber amplifier. Therefore, an additional frequency-resolved optical gating (FROG) measurement has been done to further characterize the post-pulse, which is presented in the subsequent section. A more detailed description of the fiber amplifier system can be found in, e.g., [244, 245]. Overall, the fiber amplifier system was thereby one of the key developments necessary for the trMM setup, due to the possibility of generating high harmonics with $\mathrm{MHz}$ repetition rate and thus preventing a strong pump and space charge induced secondary background and reducing the necessary integration times compared to kHz (e.g., Ti:sapphire lasers) systems. 
(a)

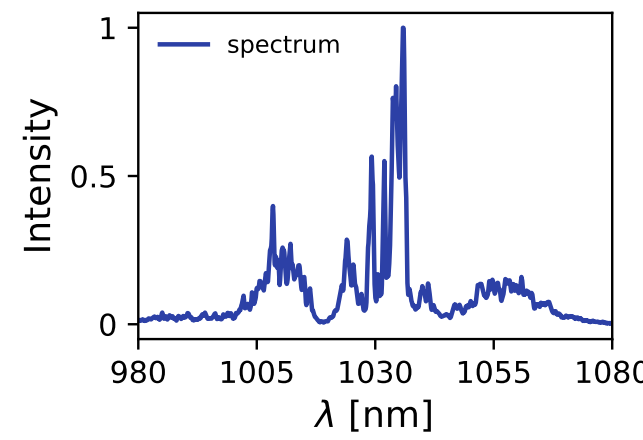

(b)

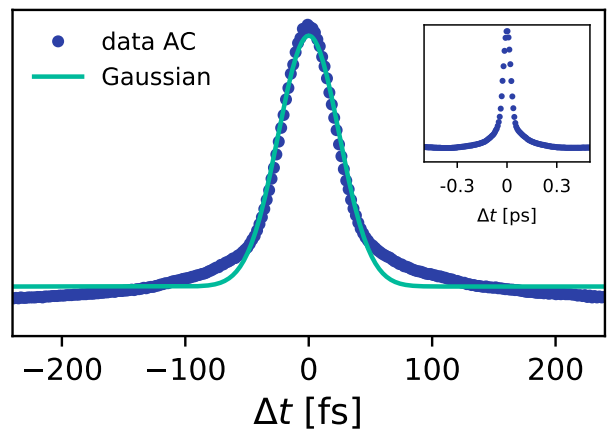

Figure 3.2.: (a) Measured spectrum after broadening in the HCF through self-phase modulation. (b) Autocorrelation measurement of the compressed pulses (blue dots) and a Gaussian fit (light blue) which does not well describe the autocorrelation, indicating a non-Gaussian pulse shape.

\subsubsection{Pulse reconstruction from frequency-resolved optical gating (FROG)}

A frequency resolved-optical gating (FROG) [246, 247] measurement has been performed to characterize the post-pulse in the fundamental of the laser amplifier system and is subsequently used in section 4.4 and figure 4.5 to determine the timeresolution of the experiment, as well as to estimate the pulse duration of the EUV probe. Briefly, in a FROG measurement the pulse is split into two beam paths where one of them is delayed in time by a delay stage. Both beams are then focussed onto a nonlinear-crystal and the resulting spectrum of the nonlinear signal is measured as a function of the time-delay $\Delta t$ between the two beams. Additional information about FROG can be found in, e.g., 246. In our case, second-harmonic-generation (SHG) FROG has been used, i.e., the second harmonic $(515 \mathrm{~nm})$ of our fundamental (1030 $\mathrm{mn}$ ) was generated, but also other configurations are possible (see, e.g., [246]). The resulting FROG traces are shown in figure 3.3 (a), together with the reconstructed pump pulse shape (b), which confirms an additional pulse around 60-70 fs. The FWHM of the central pulse measures $\approx 37 \mathrm{fs}$, assuming a Gaussian pulse shape. The post-pulse structure has been confirmed by several measurements/reconstructions. For fitting the time-dependence of the sideband generation in figure 4.5 the reconstructed pulse shape with the lowest FROG error of $G=0,0075$ was used (shown in figure 3.3 (b)). G measures the root mean square (RMS) difference of the measured and reconstructed trace [246], a value of $\mathrm{G}<1 \%$ can be regarded as a reasonable reconstruction [246, 248]. Note, however, that from the FROG measurement alone one cannot distinguish between a post- or a pre-pulse. If one takes into account the measured sideband yield (sec. 4.4) the situation becomes clear and the post-pulse can be confirmed. 
(a)

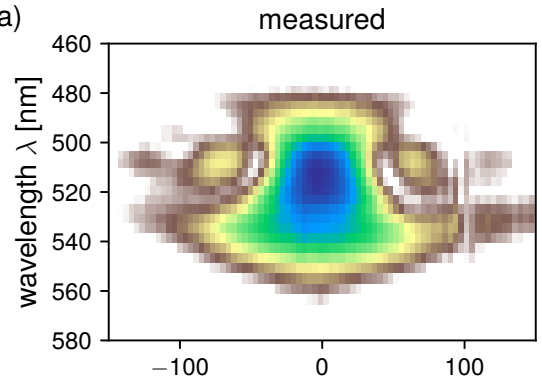

(b)

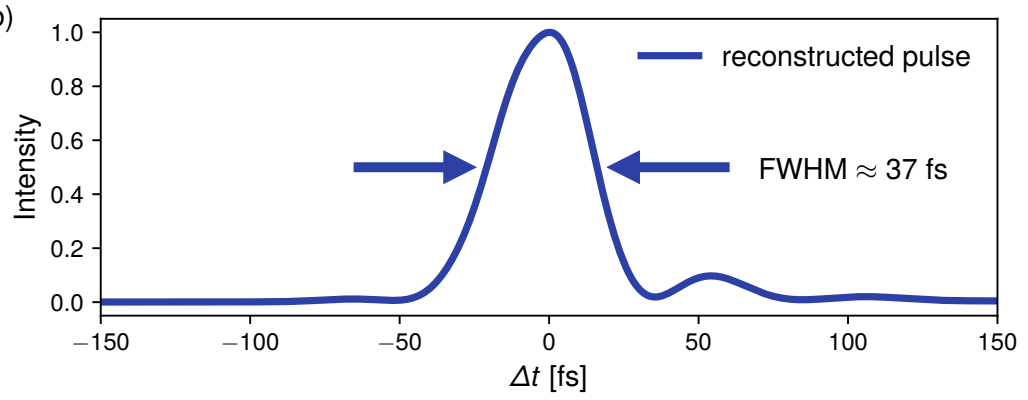

Figure 3.3.: (a) Measured and reconstructed FROG trace. From the measured FROG trace an additional pulse around 60-70 fs can be seen. (b) The reconstructed pulse shape from the FROG measurement showing an additional pulse at around 60-70 fs (FROG error of $G=0,0075$ ).

\subsubsection{High harmonic generation and EUV monochromatization}

The high harmonic generation (HHG) and EUV monochromatization scheme has been built up by Christina Möller, Marie Gutberlet [153] and Amelie Schulte [245]. It was utilized in this thesis for a time-resolved optical pump and EUV probe momentum microscopy experiment. A very detailed characterisation of the HHG source can be found in [153].

\section{The high harmonic generation setup}

For the HHG setup the compressed pulses of the fiber amplifier are used, and frequency-doubled in a beta barium borate crystal $(\beta$-BBO) to $515 \mathrm{~nm}(10 \mu \mathrm{J}$ pulse energy) before being focused in an argon gas jet to generate high harmonics [44]. Considering the high repetition rate of $1 \mathrm{MHz}$ and the resulting low pulse energies, high harmonics are generated in the tight-focusing regime with a $75 \mathrm{~mm}$ focal length lens [33, 249]. The resulting focal spot measures $12 \mu \mathrm{m}$ full-width at half maximum. The pulse duration is measured by intensity autocorrelation to be 65 fs (Gaussian), mainly due to dispersion in the thin lens and the entrance window (see section A.1 in the appendix for a typical spectrum and an intensity autocorrelation measurement). This corresponds to a peak intensity of $5 \cdot 10^{13} \mathrm{~W} \mathrm{~cm}^{-2}$ in the focus. The nozzle 
for the gas jet is mounted on a high precision 3-axes position system to optimize the gas jet position relative to the laser focus. Efficient HHG has been observed at an argon backing pressure of 2 bar for a gas nozzle diameter of $100 \mu \mathrm{m}$, which was used throughout this thesis (for the characterisation of different nozzle diameters see [153]). The gas nozzle is placed $150 \mu \mathrm{m}$ behind the laser focus where a dominant contribution from the short HHG trajectory is expected [33]. The acceptance angle of the system corresponds to a full-angle divergence of $20 \mathrm{mrad}$ whereas the EUV divergence is slightly higher. In order to limit reabsorption of the generated EUV light, the system is pumped with turbomolecular pumps backed by a multi-stage root pump. The resulting pressure with argon gas load ( 2 bar backing pressure) in the EUV generation and monochromatization chambers, which are separated by a differential pumping stage, is $2 \cdot 10^{-3} \mathrm{mbar}$ and $5 \cdot 10^{-5} \mathrm{mbar}$, respectively.

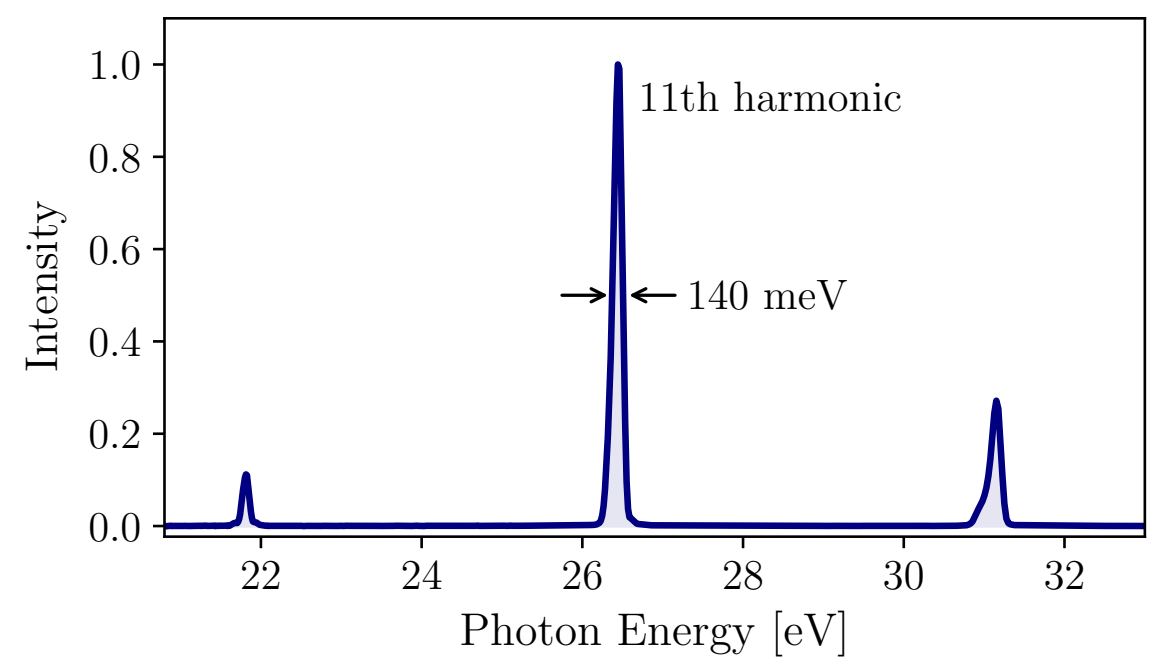

Figure 3.4.: Normalized high harmonic spectrum generated in an argon gas jet with a $10 \mu \mathrm{J}, 515 \mathrm{~nm}$ driver operating at $1 \mathrm{MHz}$. The $11^{\text {th }}$ harmonic at $26.5 \mathrm{eV}$ with a bandwidth of $140 \mathrm{meV}$ is used for photoemission spectroscopy in the momentum microscope. Taken from [78].

The HHG spectrum shown in Fig. 3.4 is measured by placing a gold mirror into the beam path just before the multilayer mirrors. The home-built spectrometer [153] consists of a toroidal mirror, a 500 lines/mm grating mounted in the off-axis geometry [250] and an EUV CCD camera. Harmonics are generated between 20 and $32 \mathrm{eV}$, separated by $4.8 \mathrm{eV}$ - twice the photon energy of the HHG driver beam. The $11^{\text {th }}$ harmonic at $26.5 \mathrm{eV}$ has a bandwidth of $\approx 140 \mathrm{meV}$ for the above specified parameters of the HHG pump pulses, which corresponds to a relative bandwidth of $\Delta E / E=5.3 \cdot 10^{-3}$. Correcting for the CCD efficiency, spectrometer and filter transmission, an average generated power of $11.5 \mu \mathrm{W}$ was estimated for the $11^{\text {th }}$ harmonic. This corresponds to $2.7 \cdot 10^{12}$ generated photons per second and a conversion efficiency of $1.5 \cdot 10^{-6}$. 
In the everyday measurement routine the phase-matching conditions were adjusted briefly to optimize the photoelectron yield in the momentum microscope and no spectrum of the used harmonics was measured due to time-constraints. This procedure has the drawback of a possible fluctuating bandwidth, which might deteriorate the energy- and momentum resolution of the momentum microscope (MM).

\section{The EUV monochromatization}

The EUV light is separated from the HHG driver by a pair of so-called grazing incidence plates (GIP) [251, which consist of a fused-silica substrate with an antireflection coating for $515 \mathrm{~nm}$ and a top-layer of $\mathrm{SiO}_{2}$. This allows for high reflection of the high harmonics ( $50 \%$ for $26.5 \mathrm{eV}$ ) at a grazing incidence angle of $10^{\circ}$ with simultaneous high transmission for the HHG driver. In order to avoid overlapping photoemission spectra excited by neighboring high harmonics, the selection of one single harmonic is necessary. This is achieved using a double mirror monochromator with two multilayer mirrors (optiX fab $\mathrm{GmbH}$ ), which reflect the $11^{\text {th }}$ harmonic around $46.6 \mathrm{~nm}$ in a $5 \mathrm{~nm}$ bandwidth. This configuration preserves the pulse duration, in contrast to single-grating monochromatization schemes which suffer from spatial chirp. The monochromator has a total transmission of $9 \%$ at $5^{\circ}$ angle of incidence on the mirrors. An extinction ratio of $\sim 1: 470$ with respect to the $13^{\text {th }}$ harmonic has been measured.

One has to note that the reflectance and thereby also the monochromatization properties of these mirrors are altered by carbon contamination, which is a wellknown problem for EUV and X-ray beamlines [252, 253]. Regular ozone cleaning with the help of an UV lamp avoids the reduction of the EUV flux [254]. The last multilayer mirror has a radius of curvature of $1200 \mathrm{~mm}$ and is positioned such that the EUV beam is focused onto the sample in the momentum microscope. A $200 \mathrm{~nm}$ thick, free-standing aluminum foil ${ }^{1}$ is used to block residual light from the fundamental beam and has a measured transmission of $12 \%$ for the $11^{\text {th }}$ harmonic, limited by thin oxidation layers on the aluminum surface. The aluminum foil is mounted in a vacuum valve, thereby separating the ultrahigh, $<5 \cdot 10^{-10}$ mbar vacuum in the momentum microscope from the high vacuum in the preceding chambers. In total, it is expected that $0.3 \%$ of the generated $26.5 \mathrm{eV}$ photons reach the sample in the momentum microscope resulting in $8.5 \cdot 10^{3}$ photons per pulse at the sample. One has to note that other schemes of the monochromatization, e.g., with metal filters only [44], can be considerably more efficient. However, the generated flux with this beamline is more than sufficient to photoemit $>1$ electron/pulse, which corresponds to the limit of the detection rate of the time-of-flight-based momentum microscope.

\footnotetext{
${ }^{1}$ For the measurements in chapter 6 a $100 \mathrm{~nm}$ thick aluminium foil has been used. This results in a higher throughput of EUV flux.
} 


\subsubsection{A versatile pump beamline}

A versatile pump beamline with tunable wavelength, pulse duration, polarization, and intensity enables pump-probe photoelectron spectroscopy on a wide range of sample systems, addressing, for example, resonant transitions in many different materials. The pump pulses are derived from the fiber laser system using a 90:10 beam splitter, positioned after the nonlinear pulse compression. The $90 \%$ output is used for HHG as described above in section 3.1.3. The residual 10\% is guided around the EUV generation and monochromatization chambers onto a delay stage to control the relative pump-probe timing. An attenuator and a $\lambda / 2$ waveplate in the pump beamline allow for manipulation of the polarization and beam intensity. Different pump wavelengths can be achieved through various nonlinear frequency conversion stages that can be inserted in the beamline, providing intense, femtosecond pump pulses at the fundamental wavelength $(1030 \mathrm{~nm})$ and its higher harmonics $(515 \mathrm{~nm}$, $343 \mathrm{~nm}, 258 \mathrm{~nm}$ ). Furthermore, a part of the uncompressed, $300 \mathrm{fs}$ laser pulses can be split off to pump an optical parametric amplifier (OPA, Orpheus-F/HP from Light Conversion). This allows to excite at wavelengths ranging from $310 \mathrm{~nm}$ up to $16 \mu \mathrm{m}$. One has to note that in the current setup, for efficient usage of the OPA, the fiber laser has to be operated at $500 \mathrm{kHz}$. After the aluminium filter, the pump beam is coupled into the MM by a mirror positioned such that the angle of the pump beam with respect to the EUV beamline is less than $1^{\circ}$ (Fig. 3.1). This prevents loss of temporal resolution due to non-collinear pump and probe beams, and ensures optimal time-resolution limited only by the individual pulse durations.

\subsection{The time-of-flight momentum microscope}

The outstanding potential of the time-of-flight momentum microscope lies in the simultaneous detection of three-dimensional data sets exceeding the first Brillouin zone and containing energy- and in-plane-momentum-resolved information on the detected photoelectrons [52, 61, 82, 85, 255, 256]. In this section, the general working principle of the time-of-flight momentum microscope will be explained (see figure 3.5). We start with a discussion of the objective lens system and the first real-space and k-space image. Subsequently the transfer lens system and the time-of-flight measurement scheme together with the delay line detector are clarified.

\subsubsection{The objective lens system}

The objective lens (see fig. 3.6 consists of the sample itself (cathode), with distance $d_{A} \approx 4 \mathrm{~mm}$ to the extractor (anode), and focussing electrodes. A high voltage between the extractor and the sample of up to $U_{A}=29 \mathrm{kV}$ is applied to facilitate the collection of photoelectrons with emission angles of up to $\pm 90^{\circ}$ for photoelectron kinetic energies up to $70 \mathrm{eV}$. Electrons that are photoemitted from the sample with a certain kinetic energy $E_{k i n}$ and momentum parallel to the surface $k_{\|}$enter the 


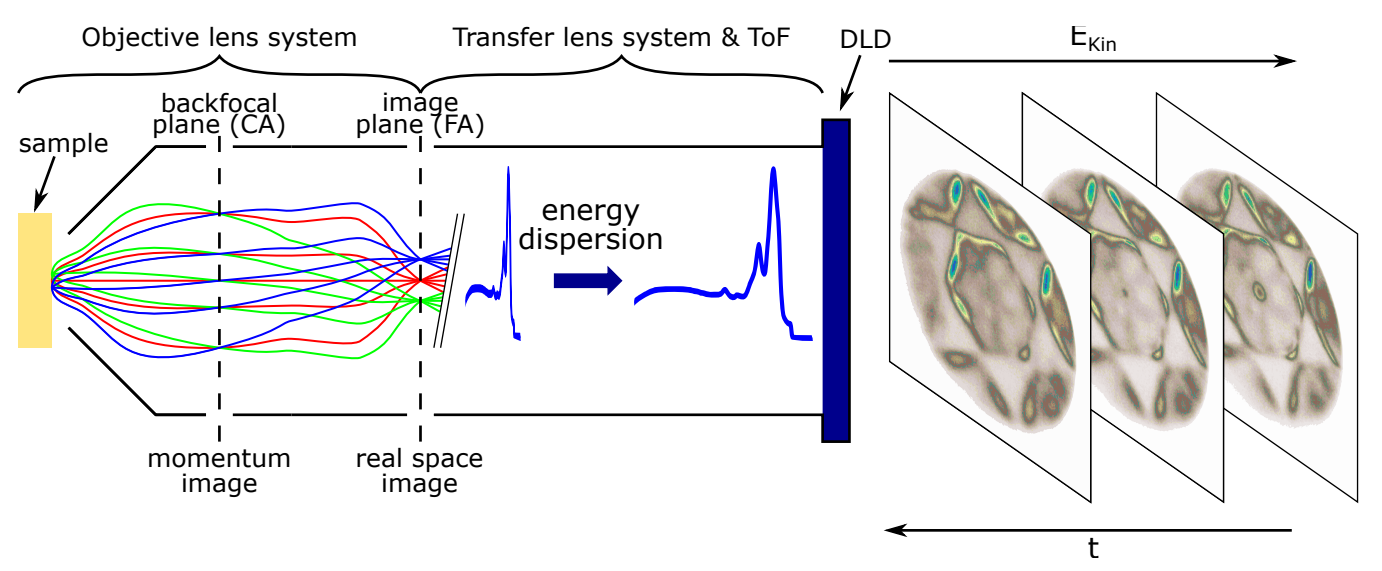

Figure 3.5.: Schematic depiction of the momentum microscope. Electron trajectories (green,red,blue) are sketched for the objective lens system which origin from different positions on the sample (After [51]). The objective lens system collects the photoemitted electrons by a high voltage between extractor (anode) and sample (cathode) $(\mathrm{kV} / \mathrm{mm})$ and forms the first momentum- and real space image in the backfocal- and image plane respectively. The transfer lens system maps and magnifies either the momentum- or real space image through the drift tube ( $\mathrm{ToF}$ ) onto a position- and time-sensitive delay-line-detector (DLD). The field-free drift section thereby ensures a temporal spread of the electrons (corresponding to a dispersion in energy), which can be resolved by the DLD. The lateral and temporal position of the photoelectrons is recorded in a 3D histogram $(\mathrm{x}, \mathrm{y}, \mathrm{t})$.

objective lens under an angle $\alpha^{\prime}$ [51, 257]:

$$
\sin \left(\alpha^{\prime}\right)=\frac{k_{\|}}{\beta \sqrt{e U_{A}+E_{k i n}}}
$$

with $\beta=\sqrt{2 m_{e} / \hbar^{2}}$ under the condition of a homogeneous electric field distribution between sample and extractor. The photoemitted electrons are only accelerated along the optical axis, conserving the parallel momentum $k_{\|}$in the process [31, 51, 257]. The first momentum image is formed in the backfocal plane of the objective lens, where different contrast apertures (CA) can be inserted into the beam path. These selections can be used to strongly enhance the contrast in the subsequent real space image, by, e.g., selecting a specific momentum feature. The lateral distance from the optical axis in the momentum image corresponds to a linear $k_{\|}$-scale independent on the kinetic energy of the electrons [31, 51, 61]. This can be understood by a short shift to classical optics. Considering a light beam which enters a lens with focal length $f$ under an angle $\alpha^{\prime}$, it is focussed in the momentum plane in a distance $d=f \tan \left(\alpha^{\prime}\right)$ from the optical axis [51]. For small angles 


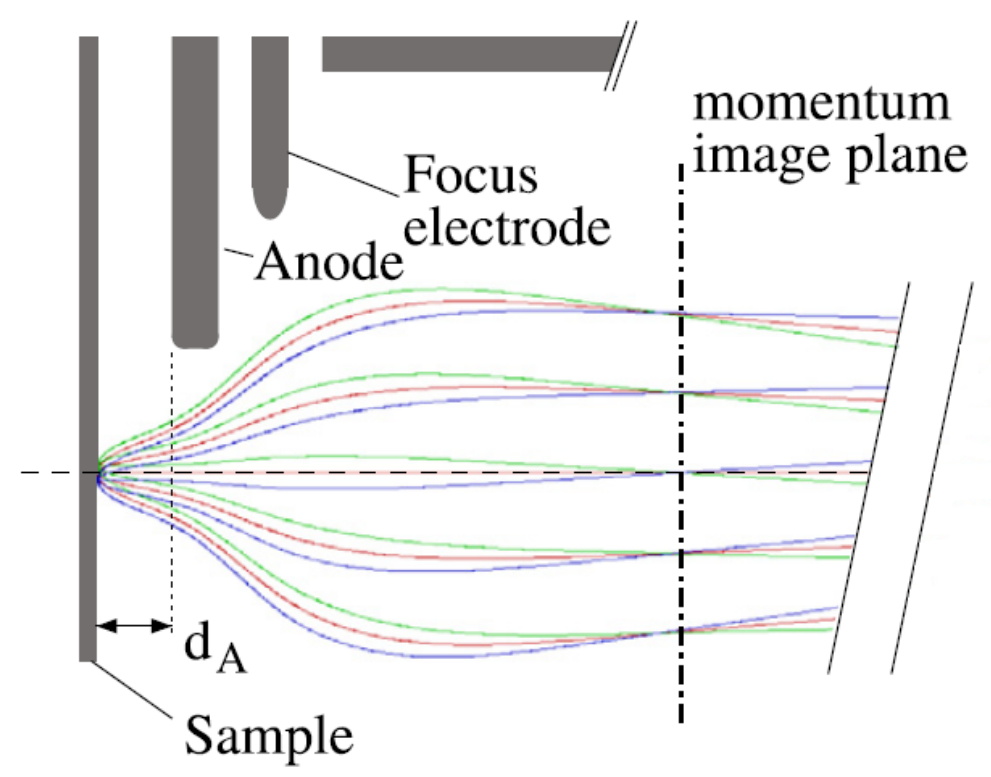

Figure 3.6.: Schematic depiction of the objective lens together with electron trajectories, which origin from different positions on the sample, adjusted from [51].

$\tan \left(\alpha^{\prime}\right) \approx \sin \left(\alpha^{\prime}\right)$ and therefore we can rewrite the distance $d$ using eq. 3.1 to:

$$
d=f \frac{k_{\|}}{\beta \sqrt{e U_{A}+E_{k i n}}} .
$$

In the approximation $e U_{A} \gg E_{k i n}$, i.e., a much high extractor voltage compared to the kinetic energy of the photoemitted electrons, the linear achromatic $k_{\|}$-scale becomes obvious [31, 51, 257]. Details on the momentum calibration, i.e., the transformation from the lateral position on the detector to momentum coordinates, can be found in section 3.3 .3 .

The subsequent real space image is formed in the image plane, where different field apertures (FA) can be inserted into the electron beam, selecting certain regions of interest in the real-space image of the sample. This allows for the easy investigation of inhomogenous sample systems (see $\mu$-APRES in chapter 7), as well as, for an easy spatial and temporal alignment of the pump-probe setup (see sec. 4.4). Furthermore, octupole stigmators are situated in the image- and backfocal plane to realign the electron beam centric on the optical axis and correct for astigmatism. Electron optical simulation identified the objective lens to be the main source of image aberration ${ }^{2}$ in the momentum microscope which can be improved using high extractor voltages [51]. Moreover, since the sample is part of the objective lens, it exemplifies how critical the alignment of the sample towards the optical axis of the extractor is. Therefore, the sample is mounted onto a motorized hexapod, which

2 Additional information about lens errors can be found in, e.g., [67, 258 261]. 
can be moved in all three directions $(\mathrm{x}, \mathrm{y}, \mathrm{z})$ and slightly tilted $\left(<5^{\circ}\right)$ with respect to the electrostatic lens system of the microscope. Additionally, a continuous-flow cryostat is integrated to facilitate cooling of the sample to below $20 \mathrm{~K}$.

\subsubsection{Transfer lens system and time-of-flight measurement}

Subsequently, a compound lens system decelerates the electron beam towards the drift voltage $U_{T o F}$, used in the time-of-flight tube, and maps either the momentumor the real space image through the drift tube onto a position- and time-sensitive delay-line-detector (DLD4040 R2.55, Surface Concept) with adjustable magnification. Additionally, three sets of coils (two at the end of the compound lens system and one before the ToF-tube), can be used to translate the electron beam and center it onto the detector.

The kinetic energy of the photoemitted electrons is deduced from a time-of-flight measurement, which is started by a transistor-transistor logic (TTL) signal from the fiber laser system, synchronized to it's repetition rate. Whenever an electron is detected by the delay-line detector it's lateral position of incidence, as well as, the time information in comparison to the starting signal are stored in a 3D $(\mathrm{x}, \mathrm{y}, \mathrm{t})$ histogram [262]. The overall travel time of the photoemitted electrons is thereby predominately determined by the used drift voltage $U_{T o F}$. From simulations, performed by Dr. Martin Ellguth (Surface Concept), the energy resolution of the time-of-flight measurement can be estimated. In this simulation only the drift tube was considered and a time resolution of the delay-line-detector of 200 ps was assumed. The resulting energy resolution $\Delta E$ as a function of the used drift voltage $U_{T o F}$ is shown in figure 3.7 (b). Obviously, this time-of-flight measurement scheme necessitates a pulsed light source, synchronized with the momentum microscope. In addition to the energy resolution, the chromatic aberration induced by the electrostatic lenses has to be considered too, which is defined for an electrostatic lens as [259 261]:

$$
\delta_{c}=C_{c} \frac{\Delta E_{k i n}}{E_{k i n}} \alpha^{\prime},
$$

with the chromatic aberration coefficient, the energetic spread $\Delta E_{k i n}$ of the electrons centred around $E_{k i n}$ and $\alpha^{\prime}$ the entrance angle of the electrons into the electrostatic lens. From here it is obvious, that the chromatic aberration will rise and the depth of focus (i.e., the sharp region in energy) will be lower for a high energetic spread $\Delta E_{k i n}$ at low kinetic energies $E_{k i n}$, as induced by a small ToF voltage. This effect can be partially corrected by restricting $\alpha^{\prime}$ using smaller apertures [85], which have to still fit to the used pump and probe beam sizes. In general, a good trade off between the used apertures, lens settings, ToF voltage and pump and probe spot sizes has to be made and customized to envisaged experiment. 


\subsubsection{Delay-line-detector}

The delay-line detector (DLD4040 R2.55, Surface Concept) used in this experiment has a typical time resolution $<180 \mathrm{ps}$, a dead time on the order of $6-20 \mathrm{~ns}$, and can resolve up to $1402 \times 1402$ spatial pixels. The general working principle of the delay-line-detectors is shown in fig. 3.7 (a). At first, the incoming photoelectrons traverse a chevron stack (see, e.g., [263] for additional information), consisting out of two multichannel plates, to amplify the signal. Subsequently, the lateral, and temporal positions of the electron cloud are reconstructed from the travel times $\left(\Delta t_{x}, \Delta t_{y}\right)$ of the induced electrical signal in the different meandering delaylines $(\mathrm{x}, \mathrm{y})$ and recorded in a 3D histogram $(\mathrm{x}, \mathrm{y}, \mathrm{t})$ [262]. Thus, by limiting the detection to one photoelectron per excitation light pulse, for each individual detected photoelectron full $\left(E, k_{x}, k_{y}\right)$-information can be gathered after calibration. Moreover, the single counting detection scheme allows to additionally record any pulse-to-pulse variations for each individual measured photoelectron, like, e.g., a timing jitter which can be used in the post-processing of the data. However, one has to note that in contrast to measurements at free-electron lasers (FELs) [82], such major post-processing is not necessary in our case due to the stability of the light source. 

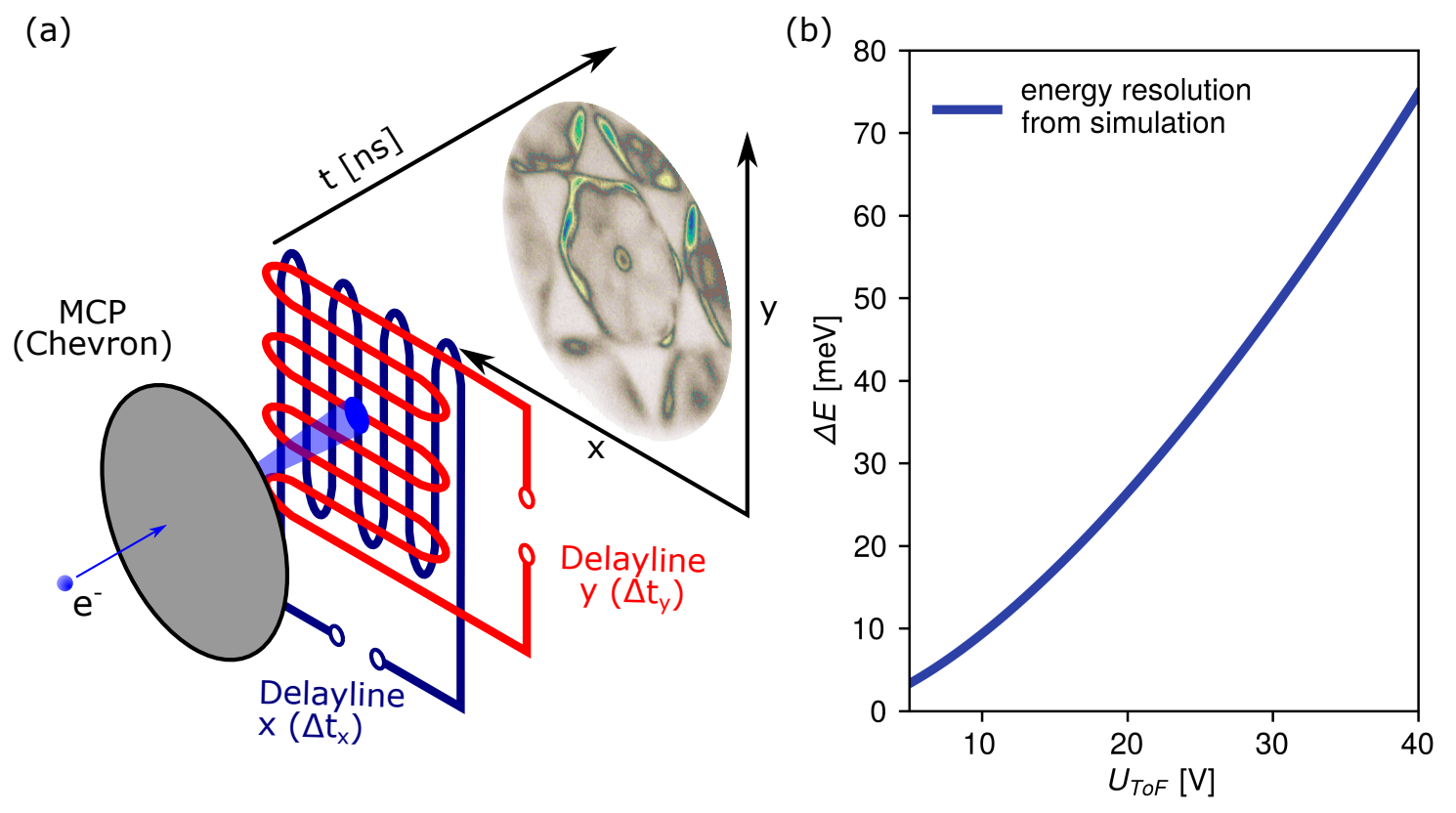

Figure 3.7.: (a) Schematic depiction of the delay-line detector (DLD). The incoming photoelectrons are multiplied in a chevron stack to amplify their signal. Subsequently the electron cloud induces an electrical pulse in both meandering delaylines $(\mathrm{x}, \mathrm{y})$, which travels to both ends of each of them. The run-time difference corresponds to the lateral position $(\mathrm{x}, \mathrm{y})$ of impact. Additionally the time-stamp t referenced to an external clock (in our case the TTL-trigger of the laser amplifier system) is recorded. After [264]. (b) Energy resolution of the momentum microscope extracted from simulations by Dr. Martin Ellguth (Surface Concept). In this simulation only the drift tube was considered and a time resolution of the delay-line-detector of 200 ps was assumed. 


\subsubsection{Analysis- and preparation chamber}

The surface sensitivity of photoelectron spectroscopy, especially in the EUV range (see fig. 2.3), necessitates an UHV chamber where samples can be prepared an checked for their surface quality. Therefore separate analysis and preparation chambers are connected to the momentum microscope which are equipped with a wide range of surface science tools for specimen preparation and characterization, including sputtering and annealing, evaporators, and low energy electron diffraction (LEED). The preparation chamber is also equipped with a load lock for fast sample exchange. Moreover, a sample storage, where multiple samples can be safely stored in UHV, has been added for a fast sample exchange. Additional information on the analysis- and preparation chamber can be found in [244, 265].

\subsection{Data post-processing}

The 3D detection scheme of the momentum microscope allows an unprecedented information depth but also poses the challenge to store and examine the data adequately, which can be seen from a simple example. Consider the size of a single data set within a time resolved measurement. One timestep $(\Delta t)$ corresponds roughly to:

$$
\underbrace{1402}_{\mathrm{x}} \cdot \underbrace{1402}_{\mathrm{y}} \cdot \underbrace{500}_{\mathrm{t}} \approx 1 \cdot 10^{9} \mathrm{px} \cdot 32 \mathrm{bit} \approx 4 \mathrm{~GB} .
$$

Therefore, a full time-resolved measurement run (e.g., 40 time steps) can easily take up 160 GB of space or more. New tools and software had to be realized for a fast and efficient data analysis and post-processing without missing on information. This was mainly achieved within the master thesis of Hendrik Nolte [266], who developed the analysis program MommyViz. In this section the data structure and some of the main steps in the data analysis are briefly introduced. For additional information about the program MommyViz see [266]. Furthermore, recent articles focussed on multidimensional photoemission spectroscopy and the subsequent data processing, which can give further insight into the topic [60, 267- 269$]$.

\subsubsection{Data structure}

The read-out of the time- and position sensitive delay-line detector (i.e., the photoelectron counts $\mathrm{I}(\mathrm{x}, \mathrm{y}, \mathrm{t}))$ are stored for each measurement in the .tif format consisting of the lateral position $(\mathrm{x}, \mathrm{y})$ and temporal position $(\mathrm{t})$ of the detected photoelectron in discretized steps (pixels). The .tif format thereby allows for a more efficient data handling (i.e., smaller data size) compared to the single event counting, which is a reasonable procedure in our case since no strong pulse-to-pulse variations are observed (see section 3.2.3). Additional metadata like laser power, delay stage position, microscope lens voltages, and driving light polarization are stored separately for each measurement run (see section A.2 in the appendix for a full overview). The 
conversion to physical quantities $\left(E, k_{x}, k_{y}\right)$ is presented in the subsequent sections.

\subsubsection{Energy calibration}

The energy calibration is inferred from the time-of-flight information of the detected photoelectrons. In principle the functional relationship between time-of-flight $\mathrm{t}$ and energy $E$ can found by, e.g., finite element simulations [67]. Here, a Taylor approximation scheme is used to find the transform between time-of-flight $t$ and energy $E$. Therefore, the energetic spectrum of the photoelectrons is shifted by known amounts in energy via an applied sample voltage $U_{\text {Sample }}$ and the time-of-flight distribution of the photoelectrons is recorded. The conversion from time-of-flight t to energy $E$ can then be expressed as (up to 3rd order):

$$
E(\mathrm{t})=E_{0}+a_{0} \cdot \mathrm{t}+\left(a_{1} \cdot \mathrm{t}\right)^{2}+\left(a_{2} \cdot \mathrm{t}\right)^{3}+U_{\text {Sample }}
$$

The coefficients $a_{0}, a_{1}, a_{2}$ are found by matching characteristic features in the photoelectron spectrum and the coefficient $E_{0}$ references the overall energy axis to, e.g., the Fermi energy $E_{F}$, which can be estimated from a Fermi-Dirac-distribution fit to the data. This procedure is shown in figure 3.8, exemplary for one $\mathrm{Au}(111)$ measurement (see chapter 6). Measurements with different sample voltages were performed $\left(U_{\text {Sample }}=17 \mathrm{~V}, 18 \mathrm{~V}, 19 \mathrm{~V}, 20 \mathrm{~V}, 21 \mathrm{~V}\right)$ with an integration time of $300 \mathrm{~s}$ each. Since this conversion is nonlinear in nature it results in non-linear pixel sizes in energy, i.e., the beforehand equally spaced time-of-flight axis is transformed to an unequally spaced energy axis, which has to be considered in the subsequent analysis.

\begin{tabular}{|c|c|}
\hline$a_{0}$ & $-0.0901 \mathrm{eV}$ \\
\hline$a_{1}$ & $0.0092 \mathrm{eV}^{1 / 2}$ \\
\hline$a_{2}$ & $-0.003 \mathrm{eV}^{1 / 3}$ \\
\hline $\mathrm{E}_{0}$ & $-9.24 \mathrm{eV}$ \\
\hline
\end{tabular}

Table 3.1.: Table showing the parameters $\left(a_{0}, a_{1}, a_{2}, \mathrm{E}_{0}\right)$ used for the energy calibration. The conversion from time-of-flight t to kinetic energies $E$ using these parameters can be seen in figure 3.8 . 

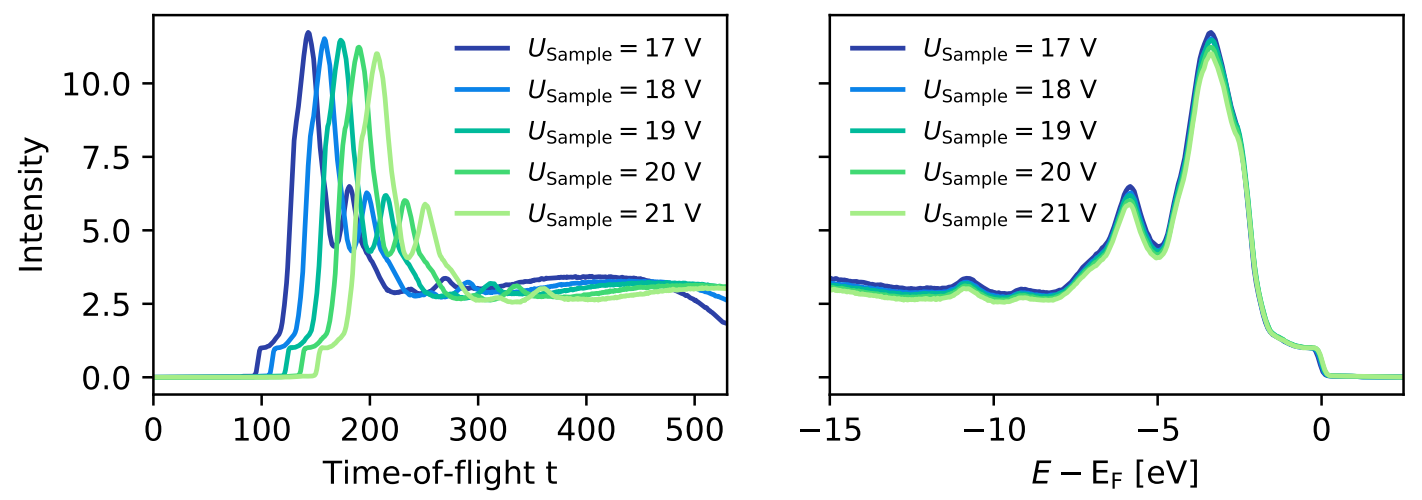

Figure 3.8.: (Left) Raw spectra integrated over the full $\mathrm{x}, \mathrm{y}$ range for the different sample voltages. Each temporal pixel corresponds to a time window of 55 ps. (Right) Spectra for the different sample voltages after performing the conversion to kinetic energies using the parameters in table 3.1. All spectra lie reasonably well on top of each other, showing that a good transformation has been found. Note that small intensity differences between the different measurements can be observed due to fluctuations in the HHG yield.

\subsubsection{Momentum calibration}

The conversion from the lateral position $(\mathrm{x}, \mathrm{y})$ on the delay-line detector to momentum coordinates $k_{\|}$is usually performed by utilizing the known size and symmetry of the surface Brillouin zone (SBZ) of the material under investigation. In this procedure the SBZ (dashed line in figure 3.9) is adjusted to the measured photoelectron distribution at a constant energy (momentum map) and known distances of high symmetry directions are compared with the measured ones (in px). This is shown in figure 3.9. Since this functional relationship is, without lens errors, achromatic (see section 3.2.1), it has to be done only once per measurement. If the SBZ is unknown, the photoemission horizon can be used to calibrate the momentum axis instead [244], whereby in a comparison between different calibration techniques for $\mathrm{Au}(111)$ the surface Brillouin zone method resulted in the smallest error compared to literature [244] and is therefore commonly used.

Here, the $\overline{\Gamma M}$ direction with a distance from the $\bar{\Gamma}$ point of $\overline{\Gamma M}=\sqrt{\frac{32}{3}} \frac{\pi}{2 a}$ is used to determine the conversion factor $\kappa$ from px to $\AA^{-1}$, using the lattice constant of $\mathrm{Au}$ $a_{\mathrm{Au}}=4.065 \AA[270$. The distance $\overline{\Gamma \mathrm{M}}$ measured $=87 \mathrm{px}$ in the momentum map (figure 3.9), so that the conversion factor can be calculated to $\kappa=0.015 \AA^{-1} / \mathrm{px}$. 


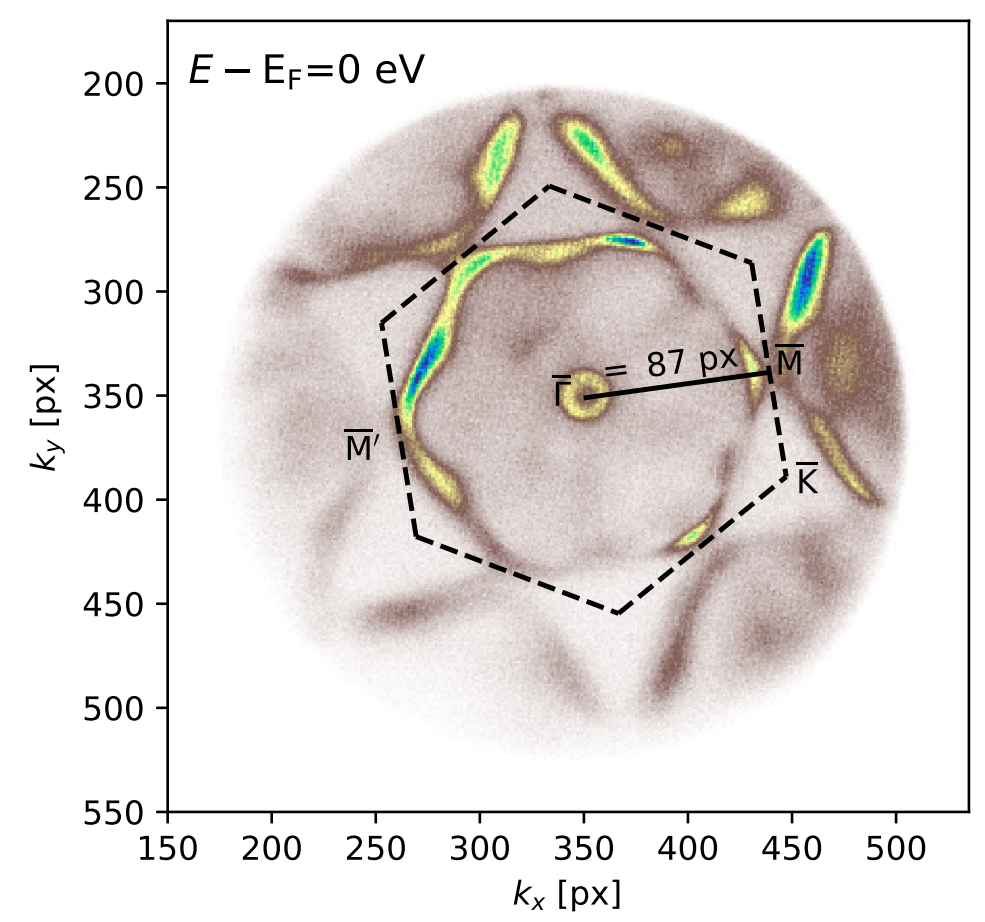

Figure 3.9.: $\left(k_{x}, k_{y}\right)$-resolved momentum map extracted from the three-dimensional momentum microscopy data on $\mathrm{Au}(111)$ (see chapter 6). The highsymmetry points $\bar{\Gamma}, \overline{\mathrm{K}}, \overline{\mathrm{M}}$ and the surface Brillouin zone (dashed) are marked. The conversion factor $\kappa$ from px to $\AA^{-1}$ can be determined from the distance $\overline{\Gamma M}=87 \mathrm{px}$.

\subsubsection{Temporal calibration}

The temporal calibration, i.e., obtaining the time-delay $\Delta t$ which corresponds to the perfect pump-probe overlap, can be found by utilizing an effect which follows directly the excitation by the driving light pulse, like, e.g., the sideband generation which is used throughout this thesis. Within temporal overlap between pump and probe, replica features of the photoemission spectrum separated by the pump photon energy are generated by the laser-assisted photoelectric effect (LAPE) and/or FloquetBloch physics (see section 2.2). The intensity of the first order sideband thereby corresponds to the cross-correlation between the pump- and probe pulses [94, 95] independent of their physical origin and can be used to estimate the overall time resolution [32, 35, 46, 131] and the individual pulse lengths (see sec. 4.4), as well as the general time-delay, which corresponds to the perfect overlap ( $\Delta t=0 \mathrm{fs}$ ). This is shown in figure 3.10 for the $\mathrm{Au}(111)$ measurement (chapter 6). In the end, after all calibrations have been performed, the individual 3D data sets for each time step are combined to a $4 \mathrm{D}$ data set $\left(E, k_{x}, k_{y}, \Delta t\right)$ representing the time-dependent evolution of the energy- and in-plane-momentum-resolved photoelectron distribution. 


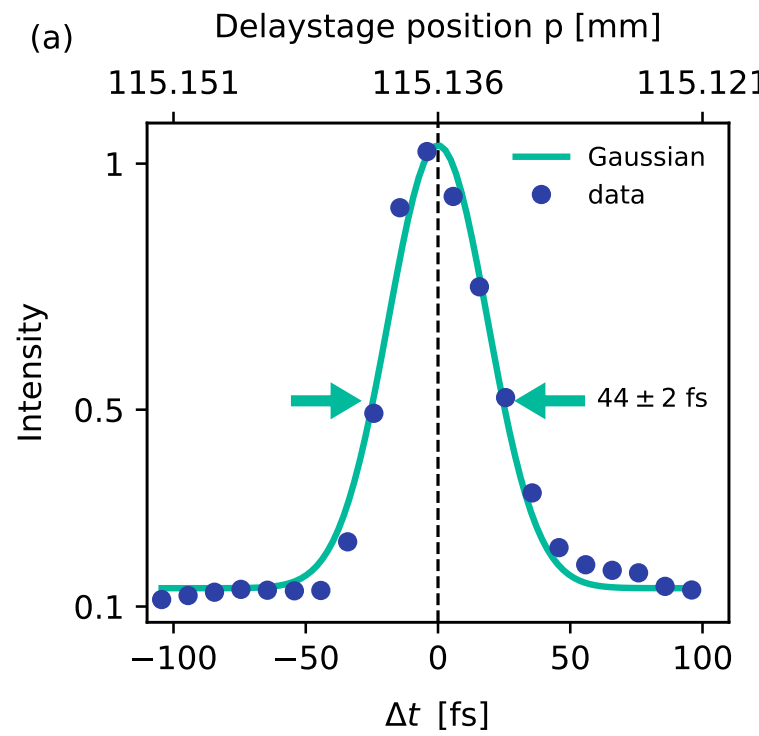

(b)

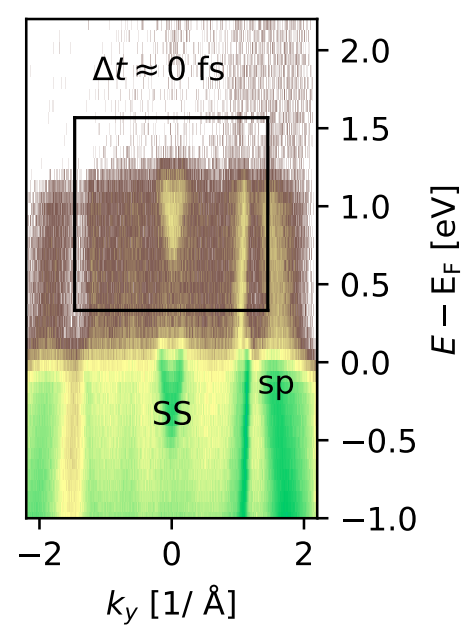

Figure 3.10.: Schematic depiction of temporal calibration of the 4D data stacks, exemplary for the $\mathrm{Au}(111)$ measurement (chapter 6). (a) The timedependent intensity from the generated sideband (marked black region in (b)) is plotted vs. the delaystage position $\mathrm{p}$ (top) and the corresponding time delay $\Delta t$ (bottom) after calibration. The calibration is performed by fitting a Gaussian to the data, whereby it's maximum is considered to be the perfect pump/probe overlap ( $\Delta t=0 \mathrm{fs}$ ). The time resolution is usually inferred from the FWHM of the Gaussian to be in this case $\approx 44 \mathrm{fs}$. A more detailed analysis, deconvoluting the cross-correlation with a measured pump pulse shape can be found in section 4.4 for a graphene measurement. (b) The $\left(E, k_{y}\right)$ resolved ARPES spectrum is retrieved by slicing the experimentally obtained three-dimensional data set in $k_{y}$ direction and integrating over a $0.13 \AA^{-1}$ large region in $k_{x}$ direction. Characteristic features like the surface state (SS) and the sp-band transition in the region below the Fermi energy $\mathrm{E}_{\mathrm{F}}$ are marked in the spectrum. A clear replica structure (sideband) is visible above the Fermi edge. Additionally a shift-correction has been performed on this data set (see sec. D.1.3). 


\subsubsection{Artefact correction}

Artefacts in the obtained 4D data sets can result from an imperfect alignment of the momentum microscope, electric or magnetic stray fields, spatial drifts (hexapod or pump/probe beams), fluctuations of the laser amplifier or the HHG process, space charge and many more. Therefore, it is important to carry out a correction scheme adapted to each individual measurement to reduce the experimental imperfections without introducing additional errors by the post-processing. Examples on the artefact correction can be found in the respective sections for the individual measurements (see section D.1.3 for a momentum shift correction, D.3.1 for the background correction of the $13^{\text {th }}$ harmonic and section B.3.2 for the symmetrization of the momentum maps). Overall, only the normalization of the time-resolved data to fluctuations in the HHG yield has been performed for all measurements and is presented here. The normalization is done by estimating the change in the measured photoemission intensity due to the fluctuating HHG, from the one-photon photoemission below the Fermi edge $E_{\mathrm{F}}$ at an normalization energy $E_{\text {norm }}$. Thereby the normalized intensity $I_{\text {norm }}\left(k_{x}, k_{y}, E, \Delta t\right)$ is calculated by:

$$
I_{\text {norm }}\left(k_{x}, k_{y}, E, \Delta t\right)=\frac{I\left(k_{x}, k_{y}, E, \Delta t\right)}{\sum_{k_{x^{\prime}}=k_{x_{\text {min }}^{\prime}}}^{k_{x_{\text {max }}^{\prime}}}\left(\sum_{k_{y^{\prime}}=k_{y_{\text {min }}^{\prime}}}^{k_{y_{\text {max }}^{\prime}}} I\left(k_{x^{\prime}}, k_{y^{\prime}}, E=E_{\mathrm{norm}}, \Delta t\right)\right)},
$$

with the raw measured intensity $I\left(k_{x}, k_{y}, E, \Delta t\right)$. This normalization scheme assumes that no other dynamics are present at the normalization energy $E_{\text {norm }}$, which has to be chosen very carefully. The effect of the normalization is exemplified in figure 3.11 for a graphene (for details on the measurement see chapter 5). An overview over different correction routines can be found in the literature, e.g., in [67, 267, 269, 271]. 

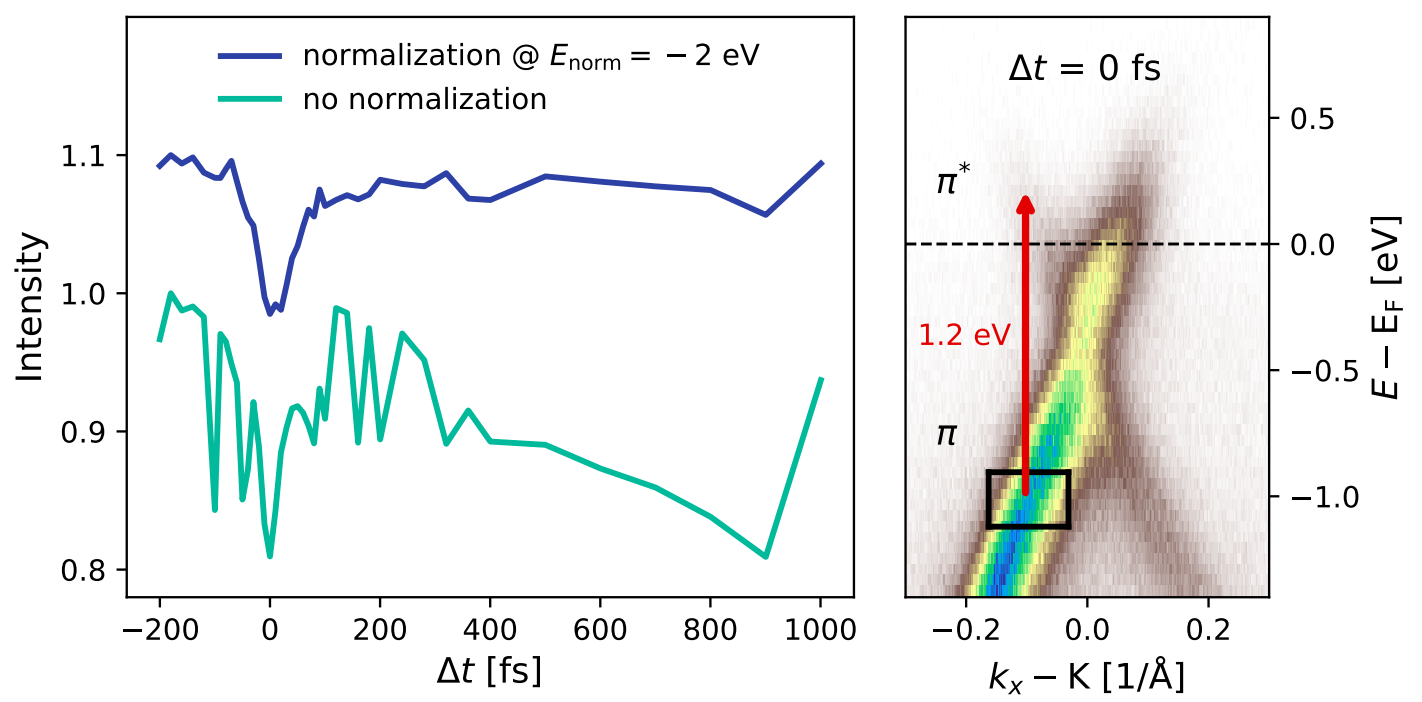

Figure 3.11.: Influence of normalization on the time-dependent dynamics. (a) The time-dependent intensity from the marked region in (b) with and without normalization is shown. For clarity the curves are shifted along the $y$-axis and their maxima are normalized to 1 . Without the normalization to fluctuations in the HHG, the hole dynamics are barely visible (green curve). After normalization the clear time-dependent behavior can be observed (blue curve). (b) The $\left(E, k_{x}\right)$-resolved spectra from graphene (for details on the measurement see chapter 5) are retrieved by slicing the experimentally obtained three-dimensional data set in $k_{x}$ direction and integrating over a $0.08 \AA^{-1}$ large area in $k_{y}$ direction. The region marked by the black rectangle is chosen such that it directly maps the holes which are created by the resonant transition from the lower $(\pi)$ band to the upper $\left(\pi^{*}\right)$ band induced by the $1.2 \mathrm{eV}$ pump pulse (the transition is marked by the red arrow). 


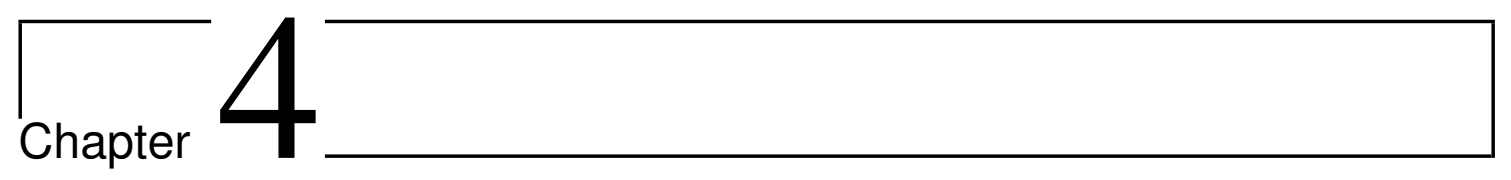

\section{Benchmarking the time-resolved momentum microscopy setup}

The aim of this chapter is to benchmark the newly built trMM setup and discuss it's advantages and disadvantages. Parts in this chapter are reprinted from the publication: M.Keunecke, et al.:"Time-Resolved Momentum Microscopy with a 1 $\mathrm{MHz}$ High-Harmonic Extreme Ultraviolet Beamline", Review of Scientific Instruments, 91(6):063905, 2020 [78] with the permission of AIP Publishing. The author of this thesis contributed as follows: The author had a significant contribution in planning and building up the trMM setup (except for the high-harmonic generation, which was planned and build up by Christina Möller, Marie Gutberlet and Amelie Schulte). He performed the corresponding measurements, data analysis, and interpretation mainly together with David Schmitt, and took part in writing of the manuscript.

\subsection{Introduction}

In this chapter the capabilities of the novel trMM setup, i.e., the combination of the table-top HHG beamline and the flexible pump beamline together with the momentum microscope, are demonstrated. We start the discussion with a characterisation of the real-space resolution, obtained from photoemission electron microcopy (PEEM) on a chessy test specimen. Subsequently the capabilities of the setup for static and time-resolved momentum microscopy are shown based on exemplary pump-probe measurements on monolayer graphene. This chapters end with a short summary and a discussion on the limitations of the trMM setup.

The performed calibrations for all the data shown in this chapter can be found in the appendix (sections B.3 \& B.4. 


\subsection{Real-space resolution}

The real-space resolution of the trMM setup has been determined with the help of a chessy test specimen, which consists of a regular pattern of Au-squares on top of a Si substrate (with it's native surface oxidation layer $\mathrm{SiO}_{2}$ ) in form of a four-fold chequerboard (see figure 4.1). Such a sample is commonly used, e.g., in scanning electron microscopes (SEM) to calibrate the magnification and check for image distortions. The chequerboard pattern has been imaged using a mercury-vapor lamp

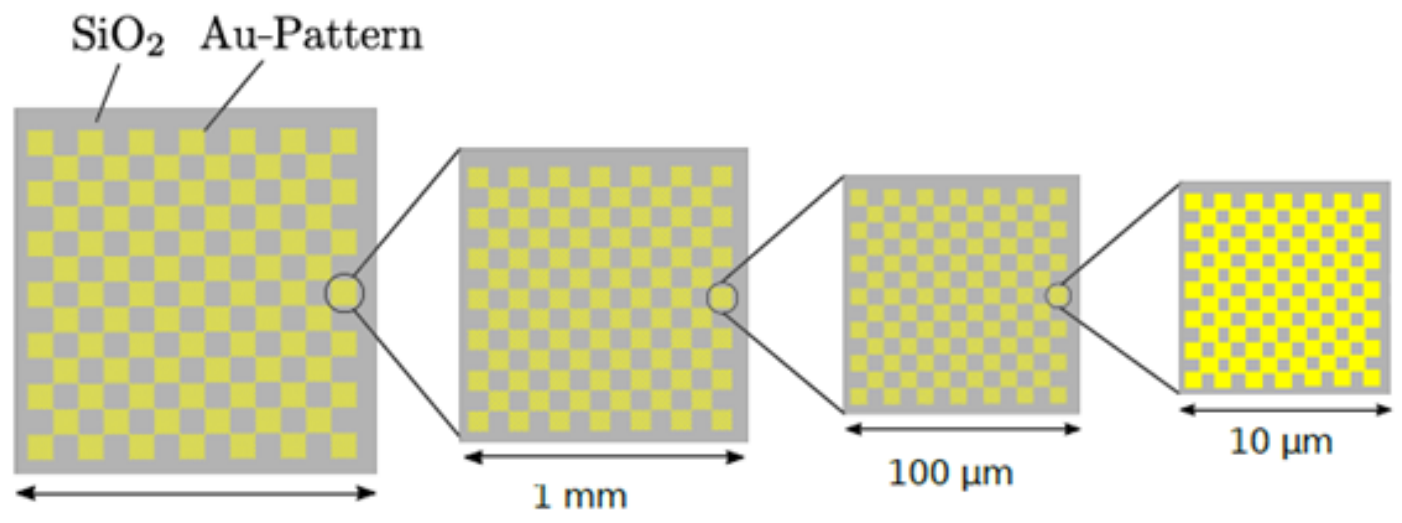

$10 \mathrm{~mm}$

Figure 4.1.: Schematic depiction of the chessy test specimen. It consists of Ausquares in form of a four-fold chequerboard from $10 \mathrm{~mm}$ in size down to $10 \mu \mathrm{m}$ on top of a Si substrate (with it's native surface oxidation layer $\mathrm{SiO}_{2}$ ). The image has been taken from [244].

at an extractor voltage of $20 \mathrm{kV}$ facilitating a high real-space magnification. The resulting image is shown in figure 4.2 (a), it displays the smallest chequerboard pattern with a field of view $(\mathrm{FoV})$ of approximately $10 \mu \mathrm{m}$. The size of the chequerboard pattern has been used to calibrate the data. A selected line profile across the edge of one Au square is shown in figure 4.2 (b). From the line profile one can estimate the real-space resolution by performing an error function fit to the data [54]:

$$
I(x)=\frac{I_{1}}{2} \frac{\operatorname{erf}\left(x-x^{*}\right)}{\sqrt{2} \sigma}+I_{0},
$$

where $I(x)$ is the measured position sensitive intensity, $I_{1}, I_{0}$ account for the intensity at the bottom and top level, $x^{*}$ is the position of the edge and $\sigma$ describes the spatial resolution. From the fit one estimates $\sigma=33 \pm 7 \mathrm{~nm}$, i.e., the FWHM spatial resolution of the momentum microscopy setup is at least $78 \pm 17 \mathrm{~nm}(\mathrm{FWHM}=2 \sqrt{2 \ln 2} \sigma)$. One has to note that this number is an upper limit for the resolution, since it assumes a perfect edge of the Au square, which is obviously not true. Moreover, no chromatic aberration of the electrons has been taken into account, which results 
from the spectral width of the mercury-vapor lamp. In figure 4.2 (c) the line profile on top of one $\mathrm{Au}$ square is plotted. Here one can notice substantial intensity oscillations which result from the architecture of the delay line detector (DLD) (see section 3.2.3). This feature can be corrected by applying a notch filter (filtering the spatial frequencies of the image distortion) or a flat-field image [244, 266].
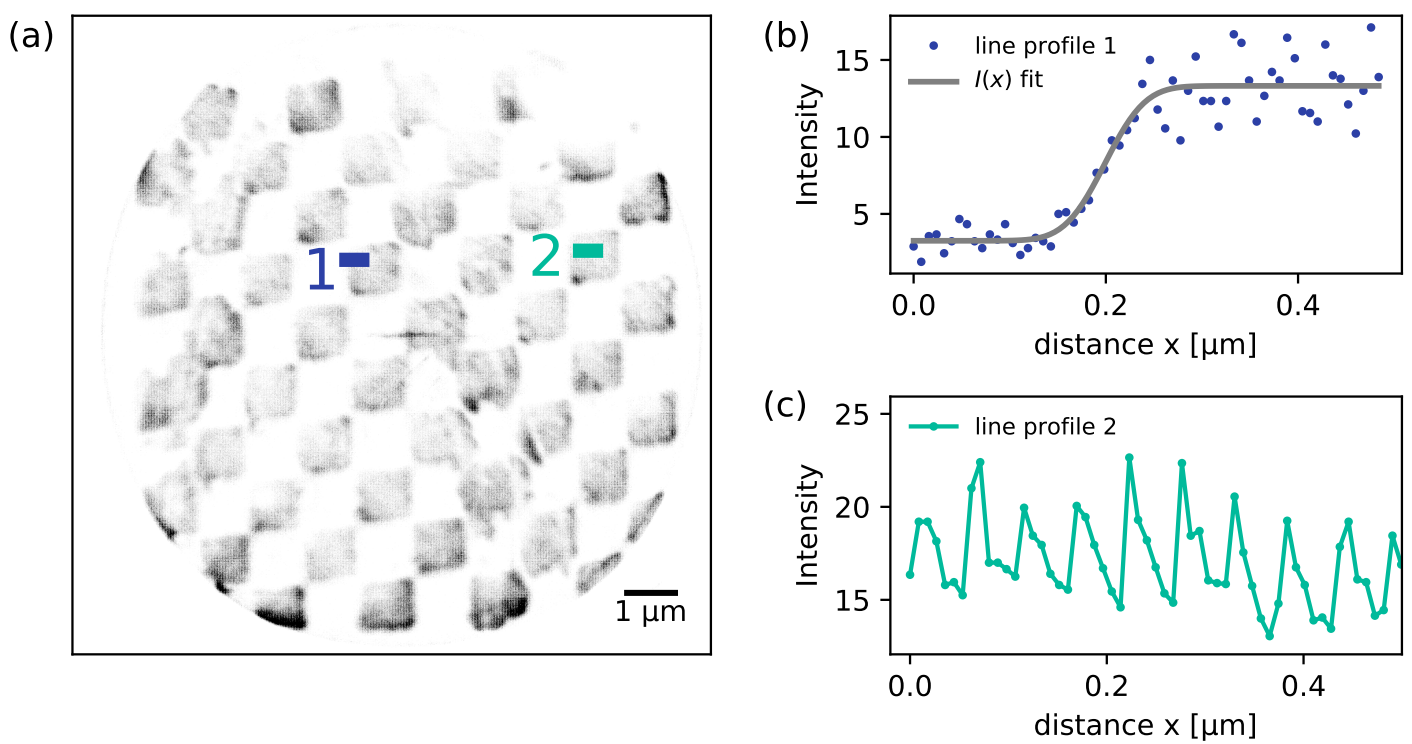

Figure 4.2.: (a) Real space image of the chessy test specimen with a FoV of around $10 \mu \mathrm{m}$. The dark squares correspond to the Au squares with a high intensity. The known size of the pattern has been used to calibrate the image dimensions. Line profiles 1 and 2 from the raw data were taken and are shown in (b) and (c) respectively, their line width corresponds to the integration area used. (b) Data from the line profile 1 (blue dots) and $I(x)$ fit to the data (grey line). From the fit we can estimate a real space resolution of $78 \pm 17 \mathrm{~nm}$. (c) In line profile 2 (lightblue) strong oscillations of the intensity are resolved which origin from the architecture of the DLD (see section 3.2.3). 


\subsection{Static momentum microscopy}

The EUV beamline combined with the momentum microscope is a versatile tool for mapping the equilibrium band structure of solid state materials as it enables the simultaneous measurement of $\left(E, k_{x}, k_{y}\right)$-resolved photoelectron distributions (see section 3.2). Very high energy- and momentum-resolution better than $<15 \mathrm{meV}$ and $<0.01 \AA^{-1}$ have already been achieved using a hemisphere-based momentum microscope in combination with a helium-discharge light source [51] (note that the same electrostatic lens system is used in our setup in combination with a time-offlight detector). In this work, the momentum and energy resolution is limited by the bandwidth of the photoemission light source, the precise settings of the electrostatic optics, and the used drift energy. An estimate of the energy-resolution of the setup in operation is presented at the end of this section.

Exemplary measurements on an n-doped graphene monolayer on a $4 \mathrm{H}-\mathrm{SiC}(0001)$ substrate $(10 \mathrm{~mm} \times 5 \mathrm{~mm})$ were performed, which was prepared by the polymerassisted sublimation growth technique [272, 273] and cleaned by subsequent heating cycles up to $600 \mathrm{~K}$ in ultra-high vacuum. Fig. 4.3 shows band mapping data from photoemission with $26.5 \mathrm{eV}$ photons to illustrate the overall performance of the setup. Satisfying signal quality within the full $\left(E, k_{x}, k_{y}\right)$-stack is achieved in an integration time of $15 \mathrm{~min}$ when the experiment is carried out at $1 \mathrm{MHz}$ repetition rate in p-polarized excitation geometry. The bottom-left plot shows a $\left(k_{x}, k_{y}\right)$ resolved energy slice taken at $E-E_{F}=2.1 \mathrm{eV}$; the first Brillouin zone of graphene can be identified by the observation of six Dirac cones at the $K$ and $K$ ' points, for which the so-called dark corridor is clearly resolved [180, 274].

Interestingly, the observed opening of the Dirac cones is not explained by the simple photoemission matrix element (Compare section 2.4.5), which points towards a final state effect similar to [180] or other possibilities. So far this topic is not yet finalized and will not further be discussed in this thesis.

From the $\left(E, k_{x}, k_{y}\right)$-resolved data stacks, arbitrary cuts in the $\left(E, k_{x}\right)$ - and $\left(E, k_{y}\right)$-directions can be extracted; Fig. 4.3 (b,c) shows such cuts along the K$\mathrm{K}^{\prime}$ and K'-K' directions. The data in Fig. 4.3 has been corrected for distortions arising from the electrostatic lens system. This is achieved using a set of stretching and shearing matrices which optimize the known hexagonal symmetry of the Dirac points [267] (see section B.3.2 in the appendix). The plane of incidence of the ppolarized EUV beam is aligned along the horizontal axis of the microscope, but $10^{\circ}$ off with respect to the $\Gamma$-K direction of the graphene sample; this twist between detector-coordinates and $\Gamma-\mathrm{K}$ is post-corrected in the data shown in Fig. 4.3. The distortion-corrected band structure agrees well with the band structure predicted by a tight-binding calculation [162]. The presented analysis highlights that the momentum microscope in operation with the table-top pulsed EUV beamline is well suited for band mapping.

For studies that concentrate on smaller in-plane momentum regions, counting statistics can be significantly improved by changing the electrostatic lens system such that only the desired region of interest is mapped onto the detector. This 
b)

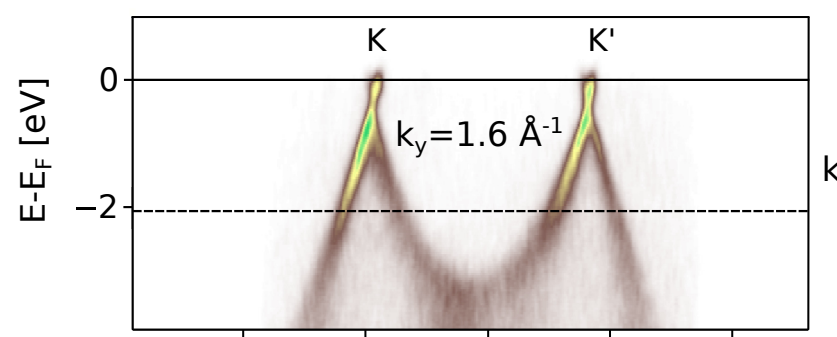

a)

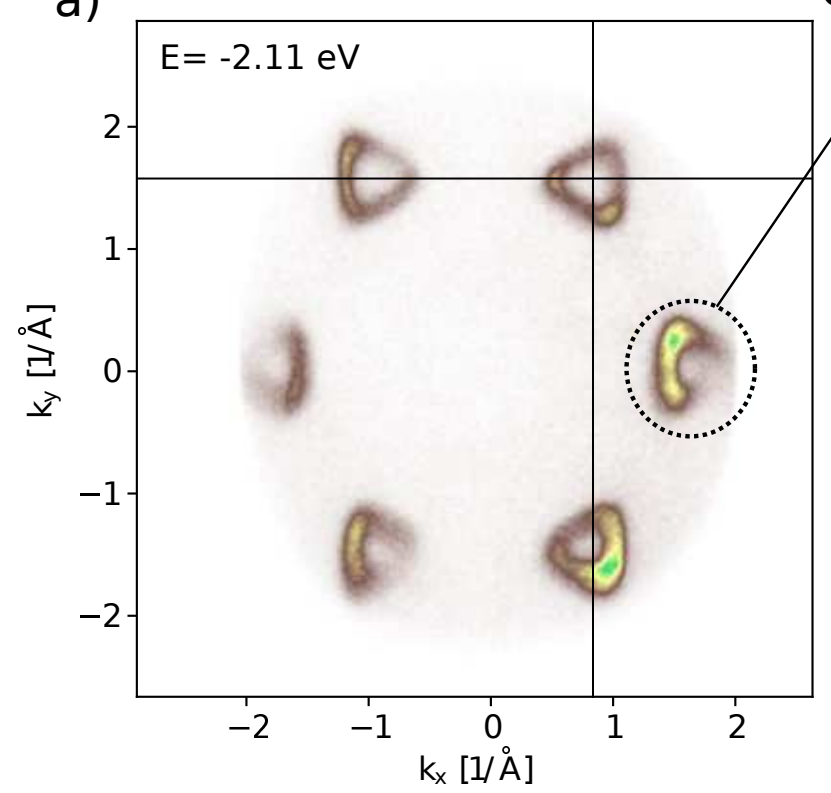

d)

c)
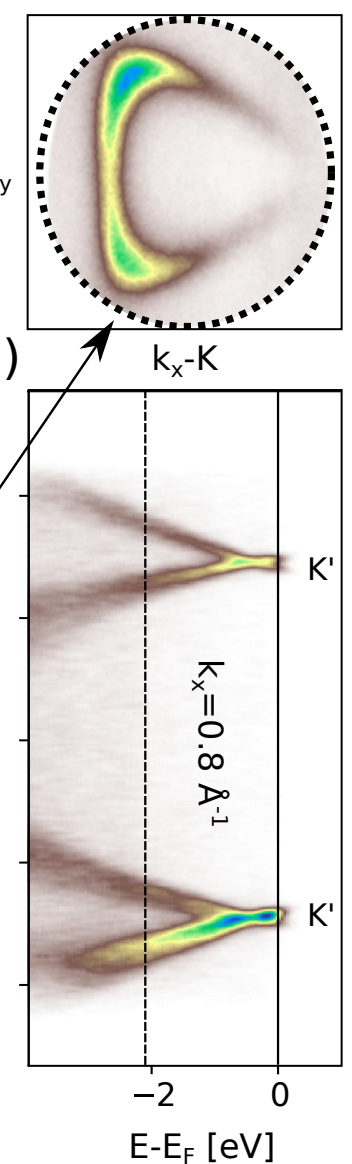

Figure 4.3.: Static photoemission spectroscopy at room temperature with $26.5 \mathrm{eV}$ photons showing the band structure of $n$-doped graphene. (a) Photoelectron momentum distribution at $E-E_{F}=2.1 \mathrm{eV}$. (b,c) Selected cuts through the $3 \mathrm{D}$ data in $\left(E, k_{x}\right)$ and $\left(E, k_{y}\right)$ direction. (d) Measurement with higher magnification settings and focus on a single Dirac cone, showing the dark corridor to the right. Taken from [78].

allows for an increase of EUV flux while staying below the detection limit of the delayline detector, effectively measuring more photoelectrons in a reduced region of interest. Fig. 4.3 (d) shows exemplary data for such a scenario, where the right-most $\mathrm{K}$ point is mapped in $\mathrm{a} \sim 1 \times 1 \AA^{-2}$ window.

From the measurements, the energy resolution of the setup is estimated by fitting photoelectron spectra with a Fermi-Dirac distribution convolved with a Gaussian function (see section B.4.4 in the appendix). In the daily measurement routine, a FWHM of $200 \pm 30 \mathrm{meV}$ is extracted for the Gaussian broadening. This broadening is composed of the linewidth of the EUV light (fig. 3.4) as well as the energy resolution of the MM, which is mainly determined by the used drift energy in the ToF (fig. 3.7 . 


\subsection{Time-resolved momentum microscopy}

The experiment is designed for the study of ultrafast non-equilibrium dynamics in the whole Brillouin zone. The performance of this combined setup is demonstrated using graphene as a model system. A brief description of the alignment of the optical-pump-EUV probe experiment is provided, and the temporal resolution of the setup is characterized.

Initial alignment of the system is performed in a microscope setting where the real-space image of the sample is projected onto the detection plane. In such an experiment, the effective beam diameters of the $1030 \mathrm{~nm}$ pump and the EUV probe pulses are determined to be approximately $100 \mu \mathrm{m} \times 230 \mu \mathrm{m}$ and $600 \mu \mathrm{m} \times 900 \mu \mathrm{m}$, respectively (see sec. B.1 in the appendix). The elliptical shapes arise due to oblique angle-of-incidence of the beams combined with astigmatism in the EUV beamline. The real-space mapping then allows the convenient optimization of the spatial overlap of the pump and EUV beams. Within the microscope, furthermore, a field aperture can be placed into the real-space image to isolate a region where spatial overlap of the pump and probe beams is guaranteed. Temporal overlap of the infrared pump and EUV probe beams can be optimized in a two-step process: First, while scanning the delay between the pump and the HHG driver beam, the multi-photon photoemission yield is monitored and optimized for maximum count rate (see sec. B.2 in the appendix). Second, temporal overlap between the pump and the EUV light is verified by optimizing the photoelectron count rate in the unoccupied states above $\mathrm{E}_{\mathrm{F}}$.

In the following, exemplary femtosecond trMM data obtained on graphene is presented. The hot charge-carrier dynamics of graphene, as well as of its bulk analogue graphite, have been studied in detail using time- and angle-resolved photoelectron spectroscopy (trARPES) [36, 179, 197, 198, 275, 278]. Typically, these experiments have been performed with hemispherical electron analyzers mapping energy- and $k_{x^{-}}$or $k_{y^{-}}$-resolved photoelectron distributions onto two-dimensional imaging detectors. Subsequent scanning of the samples azimuth angle provides access to $\left(k_{x}\right.$, $k_{y}$ )-resolved photoemission data (e.g., Ref. [179]). 
(a)

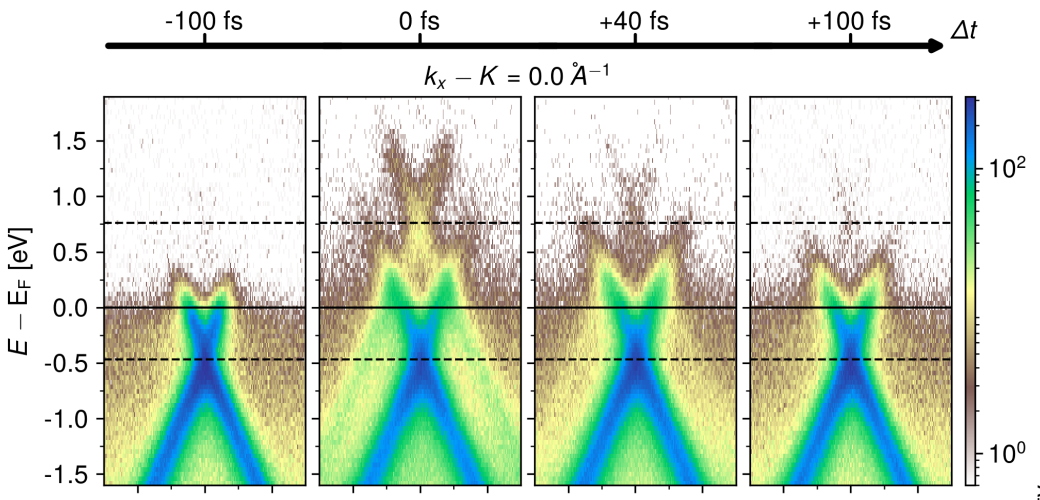

(b)
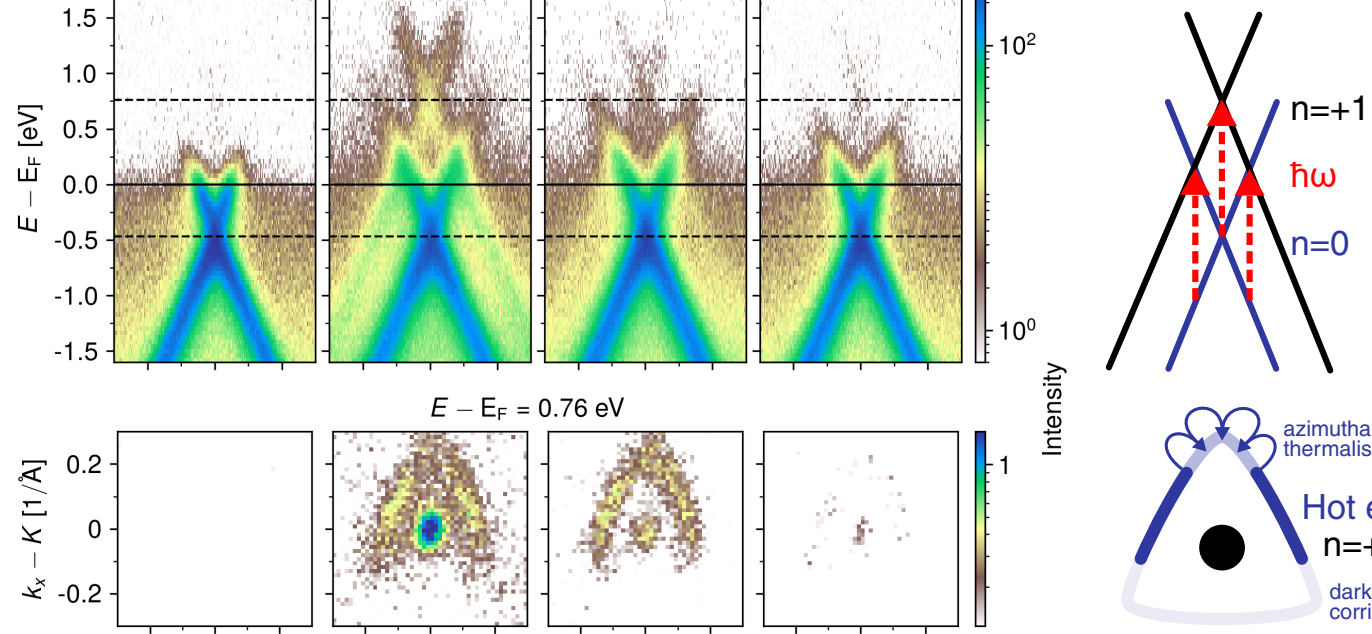

(c)
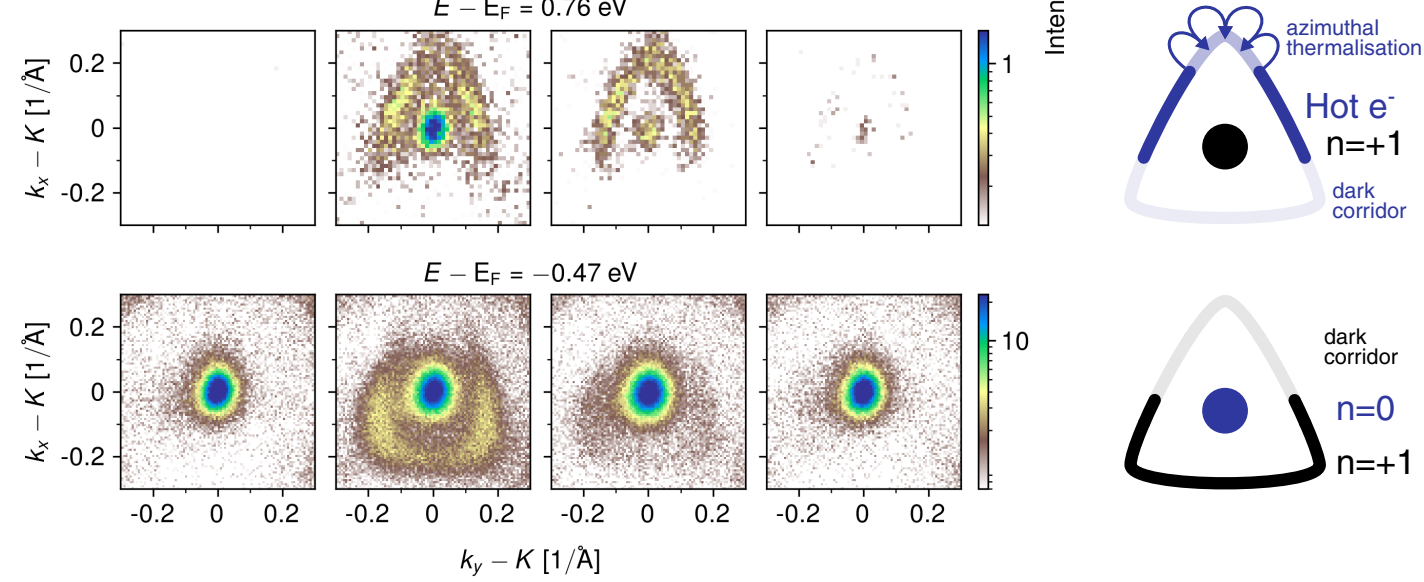

Figure 4.4.: Time-resolved momentum microscopy of graphene. (a) The ARPES spectra are obtained by slicing the $3 \mathrm{D}$ data cube at the $\mathrm{K}$ point in $k_{y}$ direction and integrating over $0.03 \AA^{-1}$ along $k_{x}$. The two dotted lines indicate the position of the momentum cuts shown in (b) and (c) respectively. At temporal overlap ( $\Delta t=0 \mathrm{fs}$ ) a clear signature of the sideband is seen, shifted in energy by the pump photon energy of 1.2 eV. Also, the anisotropic distribution of excited charge carriers within the Dirac cone [179, 180] and its relaxation dynamics is clearly visible. Taken from [78].

The trMM experiment provides simultaneous access to the non-equilibrium dynamics occurring in the energy and in-plane momentum dispersive $\pi$ - and $\pi^{*}$-bands of graphene. It has been performed in the same in-plane momentum $\left(k_{x}, k_{y}\right)$ area as in static spectroscopy (Fig. 4.3), as illustrated in Fig. 4.4. Fig. 4.4 shows selected delay-dependent $\left(E, k_{y}\right)$ - and $\left(k_{x}, k_{y}\right)$-cuts through the measured data. In these experiments, graphene was excited with the compressed output of the fiber laser $\left(1.2 \mathrm{eV}, 6.5 \mathrm{~mJ} / \mathrm{cm}^{2}\right.$, p-polarized), and probed by a time-delayed EUV light pulse (26.5 eV, p-polarized). Two dynamical processes can be identified in the data: i) Resonant excitation from the occupied into the unoccupied part of the Dirac cone leads to a non-equilibrium distribution of hot electrons, as indicated in the excitation diagram in Fig. 4.4. The thermalization of the hot charge carriers can be followed in 
real-time. ii) In temporal overlap of the pump and probe pulses, sidebands spaced by one pump photon energy from the main photoemission spectral feature are resolved. In the following, a brief description of both processes is presented, focussing on the capabilities of the trMM experiment and pointing towards related references that treat the observed phenomena.

As the ultrafast thermalization of hot charge carriers in the Dirac cone of graphene has been studied in detail by several groups [36, 179, 197, 198, 215, 275, 278, no quantitative analysis of the delay-dependent dynamics of the data is presented at this point (see chapter 5). However, a qualitative descriptions of the multi-dimensional MM data for selected time delays will be given to indicate the experimental capabilities offered by the setup (Fig. 4.4): In temporal overlap, photoexcitation with $1.2 \mathrm{eV}$ pump pulses creates an asymmetric distribution of electrons and holes in the Dirac cone, as defined by the pseudospin of the Dirac electrons with respect to the polarization of the impinging pump light [179, 215]; the time-dependent thermalization of these quasiparticles and their underlying scattering processes can be followed in real-time with high energy and momentum resolution. For excitation with p-polarized light, enhanced population of the initially unoccupied Dirac bands is localized around $k_{x}-\mathrm{K} \approx 0 \AA^{-1}$ (Fig. 4.4 (b), $\Delta t=0$ fs). With increasing $\Delta t$, azimuthal thermalization is observed ( $\Delta t=40 \mathrm{fs})$, leading to a more homogeneous charge carrier distribution that decays by electron-phonon scattering [36].

Replicated photoemission spectral features separated by the pump photon energy from the main photoemission line are of particular interest in the framework of light-induced band structure engineering, also known as Floquet engineering; the equilibrium band structure of solid state materials might be interfered by the time-periodic driving field, potentially manipulating band curvatures and even band topology [279]. However, the separation of Floquet-Bloch bands from laser assisted photoemission (LAPE) [95] is not trivial, as has been shown in trARPES data obtained on topological insulators at the $\bar{\Gamma}$-point [92, 93]. Here, these sidebands at large in-plane momenta can be resolved at the edges of the Brillouin zone with good $\left(E, k_{x}, k_{y}\right)$-resolution (Fig. 4.4). Such data facilitates the separation of Floquet and LAPE contributions to these sidebands by following the routes discussed in Refs. [92, 93] (see chapter 6).

At this point, it has to be noted that the generation of EUV light with the second harmonic [44] of the amplifier system is advantageous for trMM. Although the neighbouring $9^{\text {th }}$ and $13^{\text {th }}$ harmonics are filtered in the EUV beamline by the multilayer mirrors, residual intensity does lead to photoelectrons that are detected in our experiment. This results in replicas of the main photoemission spectral features at higher energies, spaced by $2 \times$ the driving laser frequency (not shown). For the current setup, $0.25 \%$ of the total photoemission signal is due to the $13^{\text {th }}$ harmonic. The advantage of a higher HHG driving frequency, here the second harmonic, is therefore twofold: it reduces the bandwidth requirement for the multilayer mirrors and it guarantees a maximal energetic separation of the photoemission spectral features, facilitating background free band mapping [44]. Such considerations are especially important when studying sideband formation, whose comparably weak 
photoemission signal might be overlapped with signal from a neighboring harmonic.

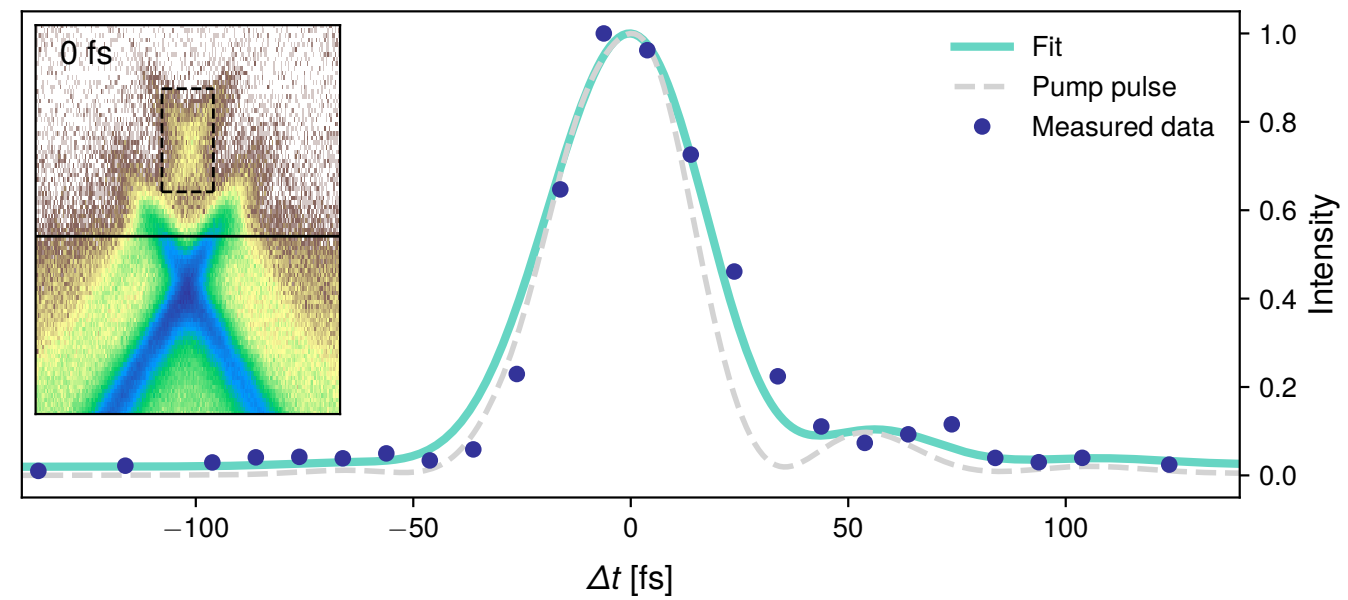

Figure 4.5.: Time-dependence of the sideband generation (blue dots), along with the independently measured pump pulse (dashed grey line) and a fit to the data (green line), in which a Gaussian probe pulse is convolved with the pump pulse. This yields a probe pulse duration of $21 \pm 5$ fs. The inset shows the measured ARPES spectrum at $\Delta t=0 \mathrm{fs}$. The box indicates the area over which the sideband signal is integrated. Note that an additional measurement has been carried out here, where the pump pulse duration has been determined and optimized using FROG (see sec. 3.1.2). Taken from [78].

Finally, one can make use of the sideband generation to characterize the timeresolution of the trMM experiment. The intensity of the sidebands, independent of their physical origin, follows the intensity of the pump laser pulse (compare section 2.2. . Therefore, one can measure the cross-correlation between the IR pump and the EUV probe pulse by integrating the photoemission signal from the sideband as shown in Fig. 4.5. The asymmetry in the time-trace arises from weak post-pulses in the pump pulse, which has been confirmed using a frequency-resolved optical gating (FROG) measurement [246, 247]. The convolution of a Gaussian probe pulse and the measured pump pulse is fitted to the measured sideband intensity. From this a full-width at half maximum of $21 \pm 5$ fs for the EUV probe is extracted.

\subsection{Summary}

In summary, a novel setup combining the capabilities of a momentum microscope with a table-top HHG, and a versatile pump beamline, all operated at $1 \mathrm{MHz}$ repetition rate has been presented. Their capabilities have been demonstrated for real space imaging (PEEM), static band mapping, as well as for femtosecond timeresolved momentum microscopy with access to dynamics in the full Brillouin zone of a material. 
The trMM setup follows up on preceding experiments using trARPES, where typically the photoelectron energy and in-plane momentum in one direction is measured. Momentum microscopes combined with time-of-flight analyzers thus offer new perspectives, because of their simultaneous measurement of the photoelectron energy and complete in-plane momentum. However, due to the novel photoelectron detection scheme, new challenges arise as well. In the following the limitations of the setup will be discussed.

\subsubsection{Limitations of the trMM setup}

Limitations of the momentum microscopy have been reviewed by multiple groups [51, 52, 61, 67, 82, 85, 280,283. Generally, the count rate and the signal-to-noise ratio of the momentum microscope are limited by the photoelectron detection scheme. Within the dead time of the delay line detector (DLD), it is not possible to detect more than one photoelectron, generally limiting the count rate to one photoelectron per laser pulse. It has been shown that segmented detectors composed of multiple DLDs can be used to increase the count rate [82. Alternatively, it is beneficial to increase the repetition rate of the excitation light source to improve the count rate. However, it is necessary to also consider aging of the multichannel plates (MCP), which becomes increasingly relevant above $10^{6}$ counts per second for the used MCP in the DLD. The typical lifetime of a state-of-the-art MCP is $>5000 \mathrm{~h}$ at $10^{6} \mathrm{cps}$ equally distributed. In terms of integral load on the MCP a doubling of the repetition rate, while maintaining the one electron per pulse regime, will thus lead to a halved lifetime. Additionally, pump-induced secondaries at small momenta can lead to an inhomogeneous degradation of the MCP, which might shorten the lifetime further. Therefore, running the experiment at $1 \mathrm{MHz}$ repetition rate with slightly less than 1 photoemitted electron/pulse is a good trade off between maximum electron count rate and detector lifetime. Also, for instrument settings where all photoemitted electrons are detected, single-electron counting on the DLD naturally avoids spacecharge effects, a common cause of signal distortion in photoemission spectroscopy 6467. 67 .

The momentum microscope is designed such that each photoelectron leaving the surface region enters the electrostatic lens system. While this is desirable in order to measure the full width of the photoemission horizon, it as well intrinsically limits the signal-to-noise ratio if only a narrow $\left(E, k_{x}, k_{y}\right)$-region is of interest in the experiment; the maximum accessible $10^{6} \mathrm{cps}$ are distributed over a larger energy and in-plane momentum region. The effective useful count rate can be improved by aligning the microscope such that only the desired momentum region is projected onto the MCP, as done for the measurement of the K-point of graphene in Fig. 4.3 (b). Complementary, the application of an energy barrier in the electrostatic lens system can block photoelectrons with low kinetic energies before they enter the time-of-flight detector [282]. In these scenarios, however, space-charge effects may arise due to the increased photoelectron density at the sample and in the electrostatic lens system up to the filtering mechanism. 
Signal distortions due to space-charge effects become even more serious when the pump pulses lead to significant photoemission. This is most-relevant with infrared to visible frequencies at higher pump fluences, where only a few photons are necessary to overcome the work function and lead to additional photoemitted electrons. Photoelectrons thus generated via the process of multi-photon photoemission [95, 284, being possibly plasmonically enhanced [285, 286], contribute to the overall count rate, limiting the effectively useful count rate in the region of interest. Furthermore, electrons emitted via secondary scattering processes or by field emission due to the large extractor voltage can give strong background signals. Those photoelectrons are typically localized at small kinetic energies and in-plane momenta and might be suppressed by placing apertures or high-pass filters, as discussed above.

Similarly, it is possible to select a different high harmonic to optimize the useful count rate. The momentum microscope collects all photoelectrons within the photoemission horizon, as defined by Eq. 2.13. Thus, by reducing the EUV energy, the counts are distributed over a smaller energy-momentum volume, facilitating an increased signal-to-noise ratio for the desired region of interest. For example, when considering an imaginary sample with a $\left(E, k_{x}, k_{y}\right)$-independent flat density of states, non-dispersive bands in $k_{\perp}$-direction, and a $\hbar \omega$-independent dipole matrix element, photoexcitation with the $9^{\text {th }}$ harmonic compared to the $11^{\text {th }}$ harmonic would distribute the count rate over a $43 \%$ smaller overall $\left(E, k_{x}, k_{y}\right)$-volume due to a smaller photoemission horizon. Note of course, that still the EUV energy has to be chosen sufficiently high to reach the in-plane momentum of the spectroscopic feature.

In pump-probe spectroscopy, it is commonly assumed that the probed system returns to the ground state well before the next pair of pump and probe pulses arrive to the sample. With increasing repetition rate of the experiment, it becomes more important to validate that assumption. In the experiments on graphene, the charge-carrier dynamics is reversible when driven at $1 \mathrm{MHz}$. However, a significant heating of the sample can be observed due to the average incident power of the pump pulses used for the excitation of the sample. This is detected using a temperature sensor which is incorporated in the copper heatsink on which the sample is mounted, meaning that the measured $60^{\circ} \mathrm{C}$ increase is most likely an underestimate of the real temperature at the active surface area for our settings. Depending on the sample under investigation, it might therefore be necessary to reduce the pump-induced heating via a reduction of the experiment's repetition rate at the cost of increased measurement time. 


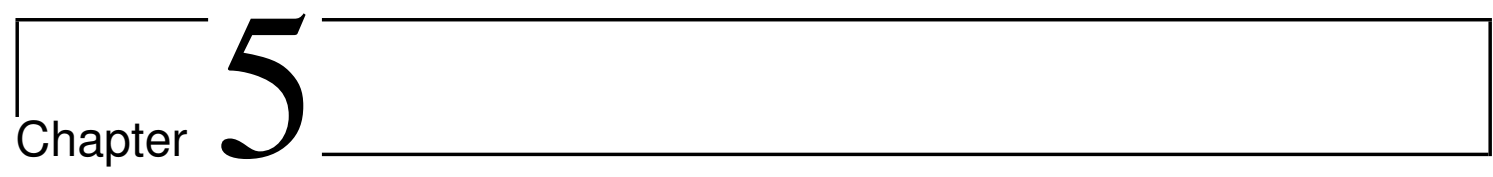

\section{Ultrafast Auger recombination in graphene}

This chapter deals with the ultrafast Auger recombination process in graphene which has so far, to the best of my knowledge, not been experimentally observed and quantized. Thereby, the new trMM setup offers simultaneous access to all relevant energies, momenta and timescales to observe and analyse the Coulomb mediated scattering processes. Significant parts in this chapter are replicated from the preprint "Direct Access to Auger recombination in Graphene" [80], whereby the author of this thesis contributed as follows: The author performed the corresponding measurements and data analysis, mainly together with David Schmitt, and took part in the interpretation of the data and in writing of the manuscript.

\subsection{Introduction}

Non-equilibrium light-matter interaction in graphene has been studied as a prototype system for fundamental energy dissipation channels of non-thermal and hot charge carriers in two-dimensional systems [36, 179, 184, 185, 189, 194, 196, 198, 200202, 205, 206, 213, 223, 224, 229, 235, 236, 239, 277, 278. In order to access these out-of-equilibrium properties on the femtosecond timescale, the optically-excited non-thermal charge carrier distribution is commonly probed using ultrafast optical [185, 189, 196, 201, 202, 205, 213, 215] and photoemission [36, 179, 197, 198, 200, 239, 277, 278, spectroscopy. Due to the linear-dispersive bands with a vanishing density of states at the Dirac point combined with the weak screening of the Coulomb interaction in the two-dimensional material sheet, many-particle electronelectron interactions are especially strong (compare section 2.5.2). As a result, the excited charge carriers thermalize to a hot Fermi-Dirac distribution on the $\approx 50 \mathrm{fs}$ timescale [196, 197, 278]. Concurrently, scattering with optical phonons leads to an azimuthal thermalization and further cooling of the Fermi-Dirac distribution on a timescale of 200 femtoseconds to a few picoseconds. Of particular interest in these carrier thermalization and cooling dynamics in graphene are impact excitation (IE) and Auger recombination (AR) band-crossing processes, whose interplay determine 
effects like carrier multiplication and population inversion. Both of them highlight the outstanding potential of graphene for optoelectronic devices [217, 287]. However, while IE has been clearly identified experimentally [200, 233], a direct experimental verification and analysis of $\mathrm{AR}$ processes remains elusive.

In this chapter, direct experimental evidence for Auger recombination (AR) on timescales as short as $10 \mathrm{fs}$ is provided, which induces an energy- and momentumdependent population far above the excitation energy. Moreover, depending on the efficiency of AR vs. other scattering processes, distinct temporal shifts are found of the transient energy- and momentum-dependent carrier populations in the conduction $\left(\pi^{*}\right)$ band. In addition, these findings are fully supported by model calculations of two-body Coulomb scattering processes in graphene performed by Marius Weber and Hans Christian Schneider (University of Kaiserslautern).

For this study an n-doped graphene sample has been selected to maximize the impact of Auger recombination on the observed scattering dynamics in the case of strong optical excitation (compare section 2.5.2 and [194, 235, 236]). Congruously, the Dirac cone is located $400 \mathrm{meV}$ below the Fermi-level. Therefore, the excitation with $1.2 \mathrm{eV}$ photons results in a non-equilibrium electron distribution with its peak located at $0.2 \mathrm{eV}$ above the Fermi-level due to resonant optical excitation $(\hat{=} 600$ meV above the Dirac point). During and subsequent to this excitation with a 37 fs laser pulse, multiple carrier-carrier scattering processes evolve and redistribute the population in energy and momentum.

Figure 5.1 illustrates the optical excitation (black arrows) and concurrent twobody Coulomb scattering processes. Here, it is important for the following discussion to distinguish in (a) band-crossing Auger recombination (AR) and multiple and inter- and intraband scattering in (b). Both scattering channels are able to generate population at energies higher than the initial optical excitation, which is the experimental signature we are going to follow, and are therefore relevant for the following discussion.

For the illustrated Auger recombination process (Fig. 5.1(a)), an electron in the conduction band $\left(\pi^{*}\right)$ recombines with a hole in the valence band $(\pi)$, and another electron in the conduction band $\left(\pi^{*}\right)$ gains the excess energy. Typical energies that can be reached in such a band-crossing Auger recombination for the scattering partner electron are $\sim 1.2 \mathrm{eV}$ above the Fermi level, if this electron originated from energies around the Fermi-level [see red arrows in Fig. 5.1(a)]. Thus, if AR is indeed of importance for the non-equilibrium dynamics in graphene [194, 235, 236], such high-energy electrons are expected to be a detectable signature of AR in this timeresolved momentum microscopy experiment. Moreover, the temporal evolution of such a high-energy electron population will give quantitative information on the the Auger recombination scattering rate itself. 


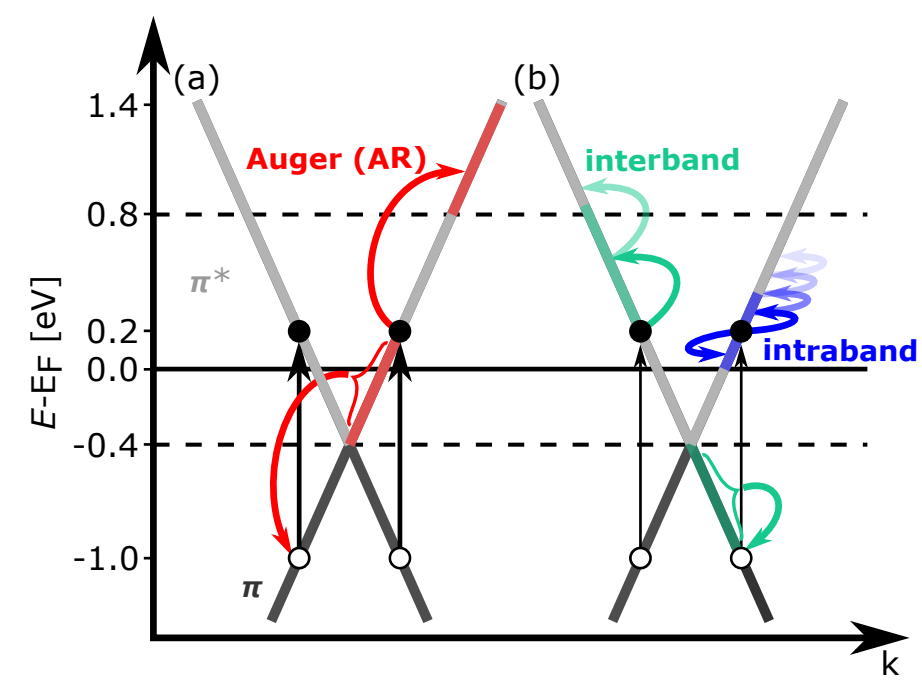

Figure 5.1.: Possible two-body e-e scattering events of non-thermal charge carriers in n-doped graphene that are able to create population at energies higher than reached by the excitation itself. Excitation with $1.2 \mathrm{eV}$ pump photons (black arrows) resonantly populates states around $E-\mathrm{E}_{\mathrm{F}}=0.2 \mathrm{eV}$ (black dots). Two processes are distinguished: (a) Band-crossing scattering processes via Auger recombination. Here, one electron bridges the valence and the conduction band and the other involved electron gains the energy of the recombination process and can reach energies up to about $1.2 \mathrm{eV}$ above the Fermi level. (b) Multiple inter- and intraband scattering processes (green and blue arrows, respectively), which can occupy higher energies in the conduction via cascaded scattering processes. Taken from [80].

However, the identification of high-energy electrons above the excitation energy might not be sufficient proof for the presence of Auger recombination processes. Upon a strong optical non-equilibrium excitation, as in this experiment, the electronic system will will instantly start to thermalize back to a Fermi-Dirac distribution, which involves multiple scattering processes, of which cascaded intra- and interband scattering processes [green and blue arrows in Fig. 5.1 (b)] are also able to generate population at higher energies in the conduction band $\left(\pi^{*}\right)$. The most important aspect is now to realize that the build-up of high-energy electron populations by multiple cascaded scattering processes versus direct band-crossing Auger recombination exhibits distinct temporal structures. In the former case, build-up of population at higher energies requires more and more individual intra- and interband scattering processes: the higher the energy, the longer it takes to create population. In contrast, for $\mathrm{AR}$, energies up to about $1.2 \mathrm{eV}$ can be reached within a single Auger scattering event, and potentially at much earlier times than expected when cascaded multiple intra- and interband scattering events are necessary to reach these energies. In total, while all these processes are possible, the available phase 
space for them critically depends on the evolution of the Dirac carrier dynamics and leads to distinct temporal, energetic and momentum signatures in the non-thermal carrier distribution.

\subsection{Measurement details}

With the goal to study electron dynamics above the excitation energy on a sub50 -fs timescale (i.e., within the timescale of the excitation pulse itself), the novel trMM setup is used to gain access to all relevant parallel momenta and kinetic energies to track the time evolution of the photoexcited charge carriers in n-doped graphene. The setup was discussed beforehand in detail in chapter 3 and 4 . In this experiment, the $11^{\text {th }}$ harmonic, corresponding to $26.5 \mathrm{eV}$, is used to probe the photoexcited carrier distribution (p-pol.). Pump pulses with $\mathrm{p}$ - and s-polarization $(1.2 \mathrm{eV}, \sim 37 \mathrm{fs})$ were used with $6.5 \mathrm{~mJ} / \mathrm{cm}^{2}$ and $17.3 \mathrm{~mJ} / \mathrm{cm}^{2}$ respectively, to excite the dynamics. Additionally, a fluence dependent measurement (p-pol., 1.5 -3.5 $\mathrm{mJ} / \mathrm{cm}^{2}$ ) has been carried out (see sec. 5.4 .2 in the appendix). The measurements were performed with an extractor voltage of $12 \mathrm{kV}$, whereby only one $\mathrm{K}$ point, located approximately in the plane of incidence of the impinging light, is imaged onto the detector facilitating an increased signal/noise ratio. A drift voltage of $40 \mathrm{~V}$ results in an overall energy resolution of $\approx 220 \mathrm{meV}$ (see figure B.7). The n-doped graphene sample on $4 \mathrm{H}-\mathrm{SiC}(0001)$ was grown by the polymer-assisted sublimation growth technique [272, 273] and cleaned by subsequent heating cycles up to $600 \mathrm{~K}$ prior to measurement. All measurements have been performed at room temperature.

\subsection{Results}

\subsection{1. p-pol. pump}

An exemplary time-resolved measurement is shown in Fig. 5.2 (a), which illustrates an $\left(E, k_{x}, k_{y}\right)$-resolved data set that is collected at $\Delta t=+10$ fs after the maximum intensity of the pump pulse (p-polarized, $1.2 \mathrm{eV}, 6.5 \mathrm{~mJ} / \mathrm{cm}^{2}, \sim 37 \mathrm{fs}$ ) is reached. Clearly visible is the linear-dispersive band structure of the Dirac cone (black dashed lines as a guide to the eye) below the Fermi-level, and also (at lower intensity) above the Fermi-level due to excitation with the $1.2 \mathrm{eV}$ pump pulse [compare also with the $\mathrm{E}(\mathrm{k})$-map in Fig. 5.3 (a)]. Note that in photoemission, some parts of the Dirac cone are not visible due to the so-called "dark corridor of graphene" (marked with "DC", check section 2.4.5 and [180, 182]). In addition, it is known and also seen in our experiment, that an excitation with $1.2 \mathrm{eV}$ laser pulses is anisotropic (indicated with black arrows for better visibility), which can be explained by a polarization-dependent matrix element for the optical excitation (compare sec. 2.5.1 and [179, 184, 204, 215]). All replica features marked with "SB" for sideband are induced by the pump pulse, as discussed beforehand in chapter 4 . These features are used here to directly determine the pump-probe overlap (i.e., $\Delta t=0 \mathrm{fs}$ ) and do 
not contribute significantly to the observed dynamics (see discussion in sec. 5.4.1).

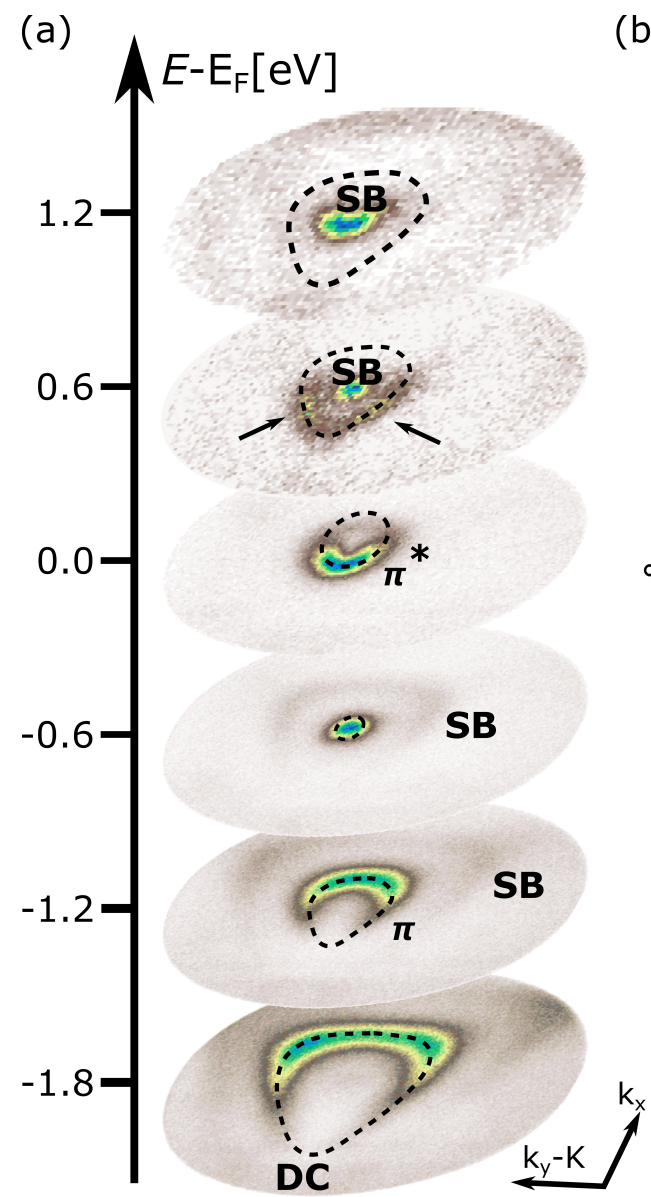

(b)
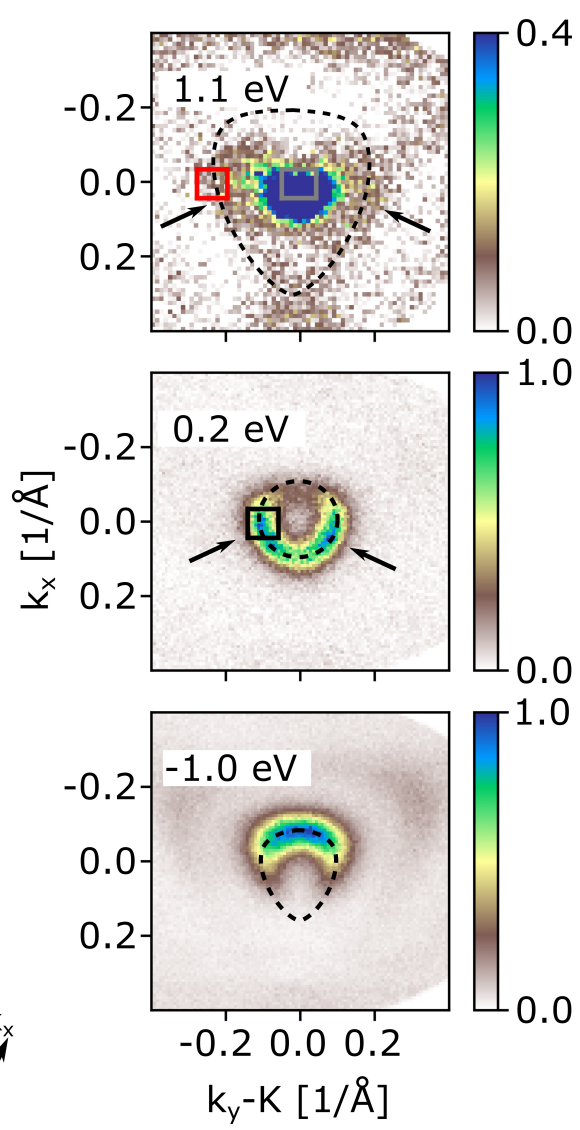

Figure 5.2.: Time-of-flight momentum microscopy data obtained on the Dirac cone of n-doped graphene when excited with p-polarized IR pump pulses and probed with EUV light $(\Delta t=10 \mathrm{fs})$. (a) 3D illustration of the collected dataset, showing the linear-dispersive valence $(\pi)$ and conduction $\left(\pi^{*}\right)$ bands (marked by dashed lines), the dark corridor (DC), the anisotropic optical excitation (black arrows), and the photon-dressed sideband (SB). The shape of the Dirac cone is indicated by a dotted line. (b) Selected $\left(k_{x}, k_{y}\right)$-momentum maps at $E-\mathrm{E}_{\mathrm{F}}=-1.0 \mathrm{eV}$, $0.2 \mathrm{eV}$, and $1.1 \mathrm{eV}$ that show cutouts from the multidimensional data set in (a). The momentum-anisotropic charge carrier distribution is conserved up to highest resolved energies $E-\mathrm{E}_{\mathrm{F}}=1.1 \mathrm{eV}$ (black arrows), implicating collinear e-e scattering events to reach these high energies. Taken from [80].

Next, the highest density of charge carriers is found in the conduction band above $\mathrm{E}_{\mathrm{F}}$ at $E-\mathrm{E}_{\mathrm{F}}=0.2 \mathrm{eV}$, as expected, because of resonant excitation by $1.2 \mathrm{eV}$ light pulses [Fig. 5.2 (b), middle panel]; as a result, the carrier distribution strongly 
deviates from a hot Fermi-Dirac distribution, both in its energy and momentum distribution. Most interestingly for our analysis is, however, that population in the conduction band is also observed for energies far above the excitation energy, i.e., up to $E-\mathrm{E}_{\mathrm{F}} \approx 1.1 \mathrm{eV}$ [Fig. 5.2 (b), top panel]. Even more, these high-energy charge carriers are also distributed anisotropically in momentum space (indicated again by black arrows). From this observation, one can already draw several conclusions:

i) As these high-energy charge carriers cannot be directly generated by the optical excitation (from the fluence dependence in the appendix sec. 5.4.2 any nonlinear contribution can be excluded), it follows that very efficient scattering events within the timescale of the pump and probe pulses must be responsible for the observation.

ii) The replication of the anisotropic distribution in momentum space is a clear signature that collinear scattering processes must dominate the generation of these high-energy electrons (black arrows).

iii) From primarily collinear scattering, as well as, the temporal and energetic region a considerable contribution of electron-phonon scattering is ruled out.

As outlined above, the sub-50-fs temporal structure of these high-energy populations is now analysed in detail (Fig. 5.3), which should be distinct for AR vs. interand intraband scattering. The analysis is performed on a momentum slice for which $k_{x}=0$, where the conduction band is directly populated in $\pm k_{y}$-direction by the optical excitation [compare black arrows in Fig. 5.2. In Fig. 5.3 (a), the respective $\left(E, k_{y}\right)$-cut is shown and the respective regions-of-interest (ROIs) are indicated with colored boxes that are further evaluated in Fig. 5.3 (b). At this point, the p-polarized pump light is used that generates photon-dressed sidebands (SB) and thus provides a direct calibration of the time axis (compare sec. 4): An analysis of the SB intensity yields a direct cross-correlation of the pump and probe pulses, implicating that maximum SB intensity is reached when they are in temporal overlap [i.e., $\Delta t=0$ fs, grey trace in Fig. 5.3 (b)]. Note that the sideband structure and the main Dirac cone cross in the energy- and momentum-region where the resonant optical excitation occurs [black box in Fig. 5.3 (a)]. However, as detailed in sec. 5.4.1, a significant contribution of this sideband yield to the measured dynamics can be ruled out.

The occupation of the conduction band as a function of $\Delta t$ is evaluated separately for each ROI indicated in Fig. 5.3 (a). Interestingly, already for $E-\mathrm{E}_{\mathrm{F}}=0.2 \mathrm{eV}$ (black ROI), where the optical excitation proceeds resonantly, maximum signal is not observed for $\Delta t=0 \mathrm{fs}$, but occurs delayed at $\Delta t_{\max } \approx 8 \pm 1 \mathrm{fs}$. This delayed response of the photoelectron yield can be attributed to finite lifetimes [288], mainly determined by e-e scattering processes which redistribute the carrier density already during the pump pulse excitation, which is in agreement with earlier reports [236]. When evaluating these time traces for increasing energies above the resonant excitation peak, the maximum population is reached at even larger $\Delta t_{\max }$ (blueish 
traces). At first glance, this is counter-intuitive in terms of excited state lifetimes, considering the relaxation of carriers towards the Fermi level, e.g,. Refs. [289, 290]. However, this behaviour must indeed be expected for the generation of this population via cascaded intra- and interband scattering: because of energy conservation and the phase space that is available for inter- and intraband scattering events, more and more scattering events are necessary to reach higher and higher energies. Consequently, $\Delta t_{\max }$ must steadily increase towards higher energies.

In contrast, however, at the highest energies where population is still detected, $\Delta t_{\max }$ decreases again (green and red trace). Therefore, these charge carriers cannot have been excited to these energies predominately via multiple scattering events. Instead, they mostly have been excited to these energies in a single scattering event, which, because of energy conservation, strongly points towards AR. The time to reach maximum population for the highest measured energy can be used for a quantitative estimation of the average Auger recombination time, where at the highest evaluated energy $\left(E_{\max }-\mathrm{E}_{\mathrm{F}}=1.1 \mathrm{eV}\right)$ a time of $\Delta t_{\max }=10 \pm 1 \mathrm{fs}$ is evaluated. This value serves as an upper limit, because the observed maximum might additionally be delayed due to finite lifetimes at the respective electron energies. Consequently, the AR time is extremely fast with $\tau_{\mathrm{AR}}<10 \pm 1 \mathrm{fs}$ as inferred from the analysis. In Ref. [200], it was found that the maximum carrier multiplication due to IE was reached within 26 fs of the optical excitation. This requires the average scattering time for IE to be well below this value, placing it also on the 10th of fs timescale. Although a direct comparison with Ref. [200] is hindered by the many parameters which influence Auger scattering times (e.g., phase space, Pauli blocking, ...), a sub-10-fs AR time seems reasonable. 
(a) $\Delta t=0 \mathrm{fs}, k_{x}=0 \AA^{-1}$

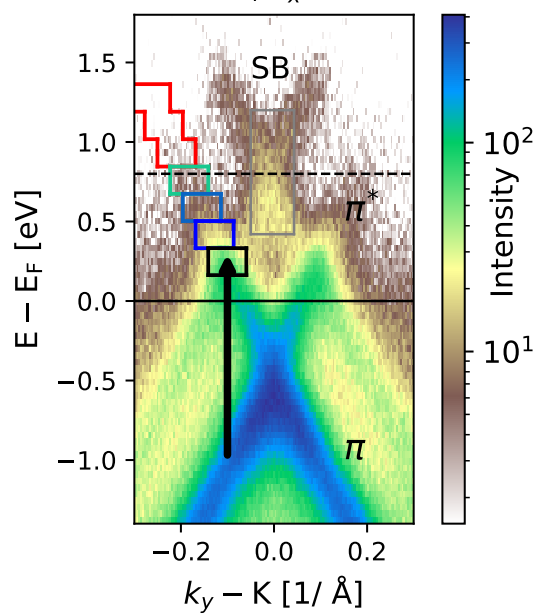

(c)

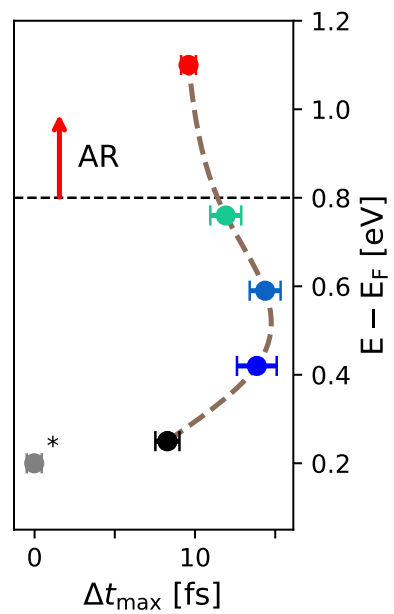

(b)

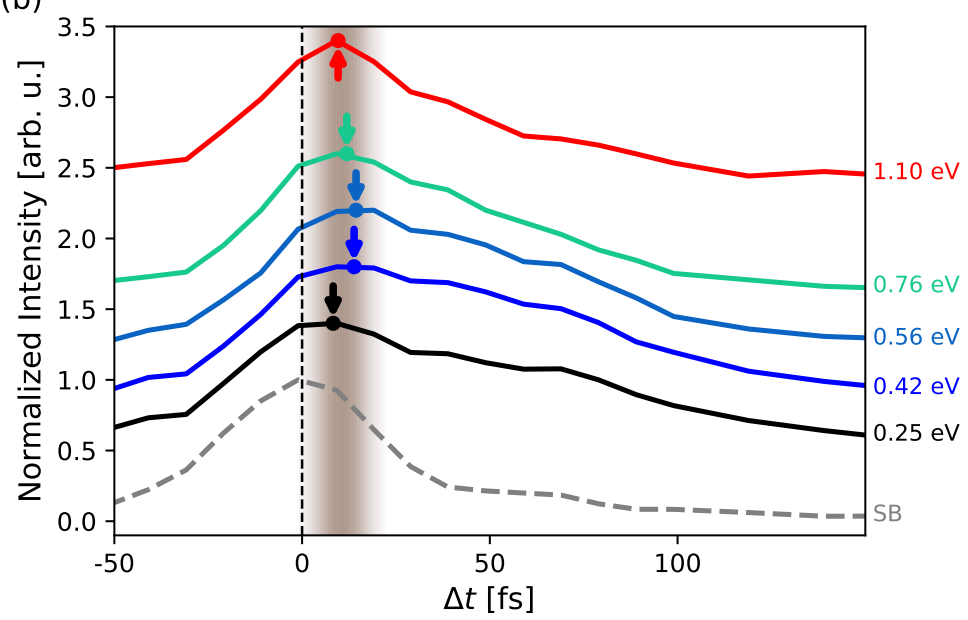

Figure 5.3.: Temporal structure of high-energy populations in the conduction band of graphene. (a) $\left(E, k_{y}\right)$-cut through the multidimensional data for $k_{x}=0$. The ROIs evaluated in (b) and (c) are indicated by colored boxes. The dotted line at $E-\mathrm{E}_{\mathrm{F}}=0.8 \mathrm{eV}$ indicates the threshold energy that can be reached with a single inter- or intraband scattering event. Higher energies can only be reached by a single AR or multiple inter- and intraband events. (b) The photoemission yield in the ROIs as a function of pump-probe delay. The time of maximum charge carrier occupation $\left(\Delta t_{\max }\right)$, obtained by Gaussian fitting of the time traces in the respective ROI between $-20 \mathrm{fs}$ and $+30 \mathrm{fs}$, is indicated by colored arrows (see section C.2). (c) Energy-dependence of $\Delta t_{\max }$; error bars correspond to the $1 \sigma$ standard deviation of the fit. For increasing $E-E_{\mathrm{F}}$, $\Delta t_{\max }$ first increases, saturates, and finally decreases again for energies where AR is expected to become the dominant scattering event. The brown dashed line is a guide to the eye through the data. From [80]. 


\subsection{2. s-pol. pump}

A similar picture as above can be gained for the data obtained with the s-polarized $\left(1.2 \mathrm{eV}, 17.3 \mathrm{~mJ} / \mathrm{cm}^{2}\right)$ pump light as shown in figure 5.4 . The data is evaluated as done in section 5.3.1 for the p-polarized case (the maxima determination is discussed in section C.2. . Consistently, a distinct shift of $\Delta t_{\max }=9 \pm 1$ for the high energetic carriers $(1.1 \mathrm{eV})$ is observed, which is interpreted as a direct evidence for AR. The overall shift of $t_{\max }$ shows qualitatively and quantitatively a similar energy dependence (within error bars). Note, that now in (a) a cut along the $k_{x}$ direction is shown, to follow again the direct excitation by the pump beam (which is rotated by $90^{\circ}$ from p- to s-polarization) and additionally only one arm of the Dirac cone is nicely visible along this direction in photoemission due to the dark corridor (see section 2.4.5.

The overall levelling in the data (i.e., the flat plateau around $\Delta t=0 \mathrm{fs}$ ) can be attributed to increased Pauli-blocking (state-blocking) due to the higher fluence in this measurement $\left(17.3 \mathrm{~mJ} / \mathrm{cm}^{2} \gg 6.5 \mathrm{~mJ} / \mathrm{cm}^{2}\right)$, which fits also to the theoretical expectations (compare section 2.5.1). This hampers slightly the determination of the maximum population leading to higher errors in this measurement (see section C.2 in the appendix). 


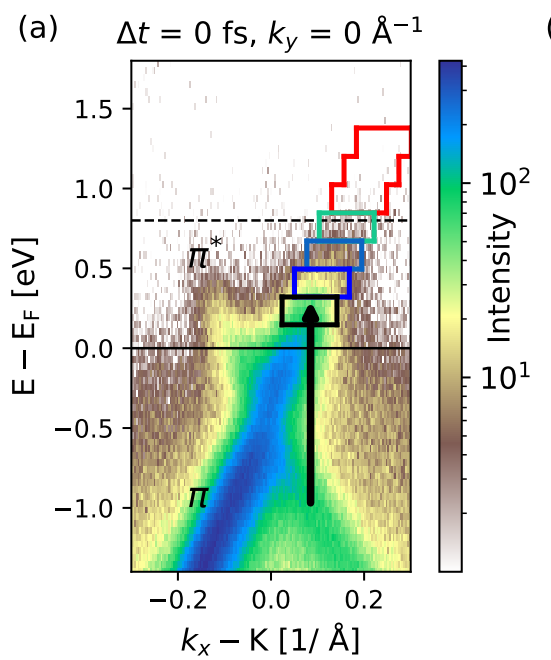

(c)

(b)
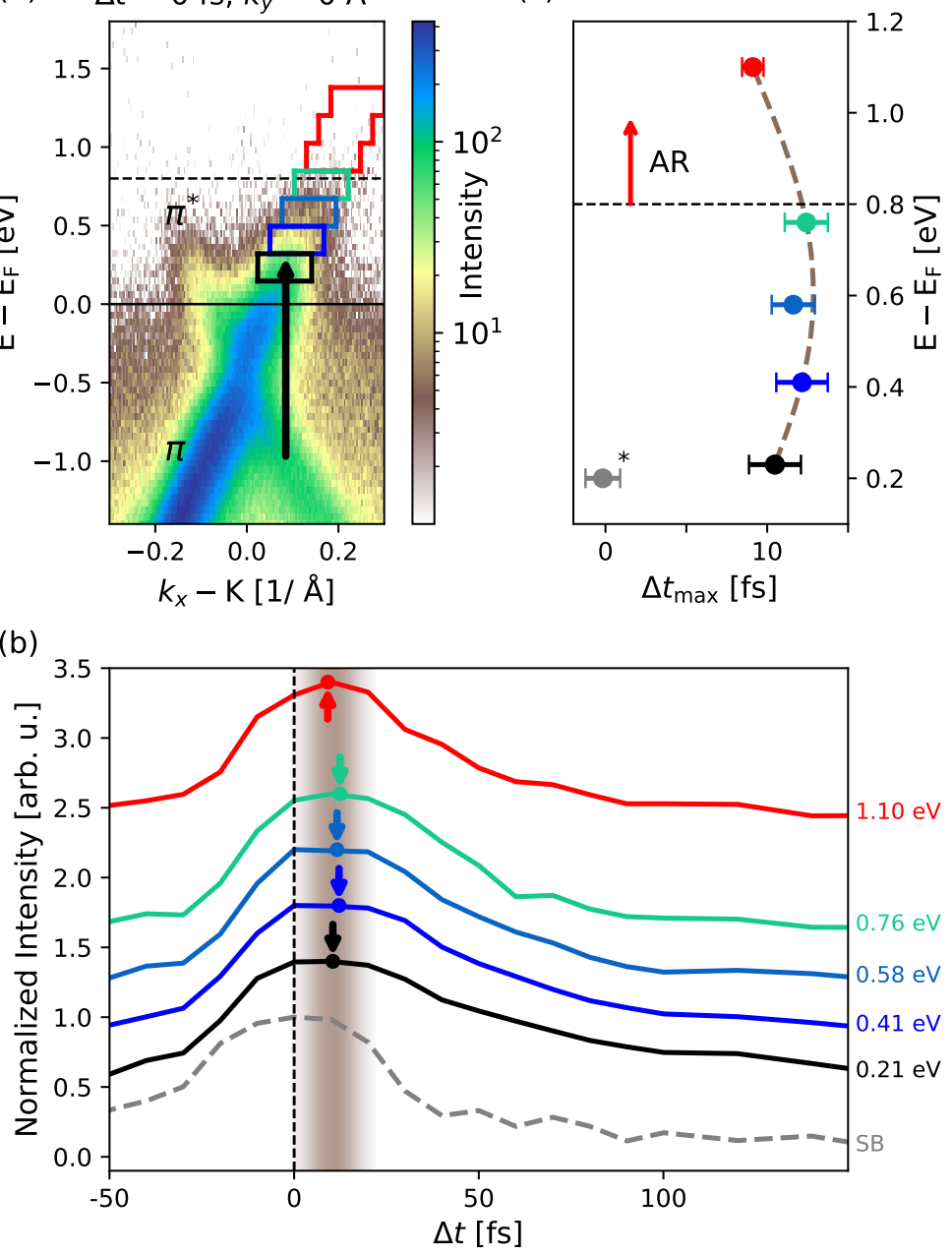

Figure 5.4.: Identification of AR in the ultrafast, energy- and momentum-resolved, redistribution of non-thermal charge carriers in the conduction band of graphenet for s-polarized pump light. (a) $\left(E, k_{x}\right)$-cut at $k_{y}=0$. The regions of interest evaluated in (b) and (c) are indicated by colored boxes. The dotted line at $E-\mathrm{E}_{\mathrm{F}}=0.8 \mathrm{eV}$ indicates the threshold energy that can be reached with a single inter- or intraband scattering event. (b) The photoemission yield in the ROIs is evaluated as a function of pump-probe delay. The time of maximum charge carrier occupation $\left(\Delta t_{\max }\right)$ in the respective ROI is indicated with a colored arrow. Note that the SB labelled time trace is obtained from a weak sideband signal. (c) Energy-dependence of $\Delta t_{\max }$ obtained by Gaussian fitting of the time traces between -20 fs and $+30 \mathrm{fs}$; error bars correspond to the $1 \sigma$ standard deviation of the fit. The sideband trace (grey) is used for the calibration of the time-axis (note that its plotting on the $E-\mathrm{E}_{\mathrm{F}}$-axis is not physical and therefore marked with a $*$ ). For increasing $E-\mathrm{E}_{\mathrm{F}}$, $\Delta t_{\max }$ first increases, saturates, and finally decreases again. The brown dashed line is a guide to the eye through the data. Taken from [80]. 


\subsubsection{Model calculations}

Simulations of two-body Coulomb scattering processes in graphene were carried out by Marius Weber and Hans-Christian Schneider (see appendix C.4 for details). Results of these simulations are shown in Fig. 5.5. To keep the simulation and comparison simple, the dynamics in the simulation were initiated ( $\Delta t=0 \mathrm{fs}$ ) by anisotropically redistributing the charge carriers between the conduction and the valence band. The resulting imbalance of electrons and holes at $E-E_{F}=0.2 \mathrm{eV}$ and $-1.0 \mathrm{eV}$ corresponds to the experimental excitation, as shown in the inset of Fig. 5.5 The main part of Fig. 5.5 shows transient electron populations for $E-E_{F}>0.8 \mathrm{eV}$ calculated for two cases: (i) including all Coulomb-mediated e-e scattering pathways, and (ii) for an artificial case neglecting all scattering events in which one carrier crosses bands. Thus in scenario (ii), AR processes are excluded in the simulation. The simulation shows that the total carrier density at $E-E_{F}>0.8 \mathrm{eV}$, i.e., at energies beyond those reached by the pump pulse rises after the excitation to a value that is only a small fraction of the total carrier density in both cases. A subsequent decrease of the density in this energy range is not observed because electron-phonon interactions are not included that would lead to such a "cooling" behavior. However, the direct comparison clearly shows that AR processes play a large enough role at high energies such that its signatures can be picked up by the experiment. While the timescales are slightly longer than those in the experiment due to the choice of the screening parameter, it can be concluded from the calculations that it is the large influence of AR processes on the high-energy dynamics that makes them detectable, even though the total carrier density at high-energies is extremely small.

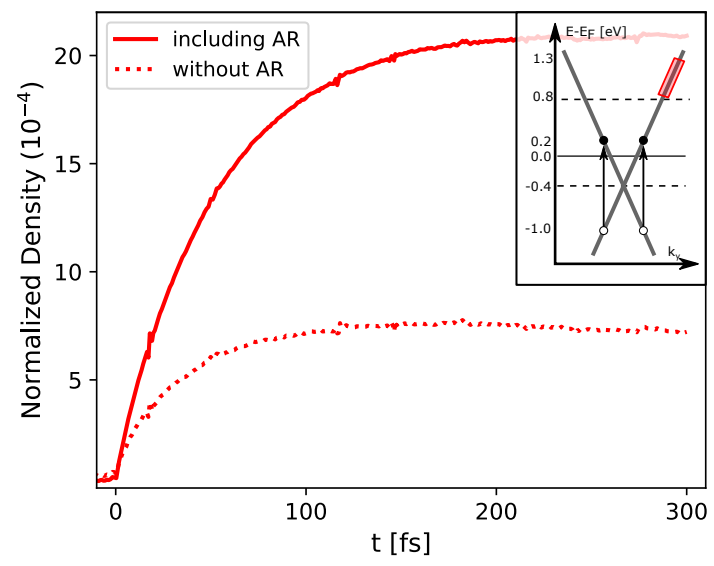

Figure 5.5.: Computed time-dependent carrier densities integrated over the energy range $E-E_{F}>0.8 \mathrm{eV}$ corresponding to the experimentally accessible population of high-energy electrons. The density is normalized to the total density of electrons in the conduction band. The red solid line is computed including all scattering channels and the dashed line excluding Auger processes. Taken from [80]. 


\subsection{Discussion}

\subsubsection{Excluding the influence of sideband intensities on the carrier scattering analysis (p-polarized pump light)}

From Fig. 5.3 (a), one can see that the sideband trace crosses the main Dirac cone where optical excitation proceeds resonantly (black ROI). The temporal overlap between pump and probe ( $\Delta t=0 \mathrm{fs})$ is calibrated in the energy- and momentumregion where the sideband does not overlap with the main Dirac cone (grey ROI). In Fig. 5.3 (b,c), the grey ROI and the black ROI have distinctly different $\Delta t_{\max }$. Such a scenario is not be expected if significant contributions of the black ROI would stem from sideband yield; instead, the dynamics measured in the black ROI are dominantly determined by the charge carrier dynamics in the conduction band. If still considering minor contributions of the sideband to the black ROI, $\Delta t_{\max }=$ $8 \pm 1$ fs has to be treated as the lower limit.

Moreover in the s-pol. measurement only a vanishing sideband intensity is detected. This can be understood, due to probing of the Dirac cone approximately located in the optical plane, and thus for s-polarized pumping, no contributions from the laser-assisted photoelectric effect (LAPE) are expected (compare sections 2.2 and 6. The fact that the s-polarized data and analysis in Fig. 5.4 shows the a similar energy-dependent delay further supports that the intensity of the sideband in the p-polarized case does not contribute significantly to the analysis procedure.

\subsubsection{Excluding higher-order nonlinear contributions}

Fluence dependent measurements have been performed with the p-polarized pump beam to rule out strong nonlinear contributions for the electrons at higher energies $(\geq 0.8 \mathrm{eV})$, i.e., a two photon absorption or higher order sideband generation. Here, the observed sideband is used as an additional check, since it is expected to scale linearly with pump fluence (compare sec. 2.2). In figure 5.6 (a) the momentum map centred at $0.8 \mathrm{eV}$ (obtained by integrating from $0.7 \mathrm{eV}-0.9 \mathrm{eV}$ ) for the measurement with a fluence of $\sim 3.5 \mathrm{~mJ} / \mathrm{cm}^{2}$ is shown together with the corresponding integration areas for the carriers in the $\pi^{*}$ band (blue) where a resonant two photon absorption can expected. Note, that the integration area has been enlarged compared to figures 5.3 and 5.4 to account for a reduced signal/noise ratio, especially at lower fluencies. In (b) the fluence dependence of the sideband generation $(0.8 \mathrm{eV}-1.4 \mathrm{eV})$, the carriers at high energies in the $\pi^{*}$ band (due to AR, $0.8 \mathrm{eV}-1.4 \mathrm{eV}$ ) and the carriers at the energy for the resonant two photon absorption $(0.7 \mathrm{eV}-0.9 \mathrm{eV})$ in temporal overlap $(\Delta t=0 \mathrm{fs})$ are shown in a log-log plot. The corresponding intensities have been corrected for background counts by selecting a similar ROI with the same area at the same energy in the background. The intensities in all three areas follow approximately a linear fluence dependence ruling out strong non-linear contributions by high-order processes. As expected, the sideband intensity scales with a slope of $1.0 \pm 0.1$, confirming the linear dependence and verifying the background correction. 
(a)

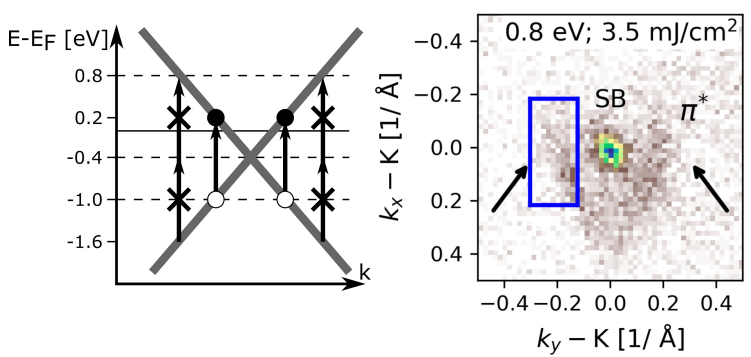

(c)

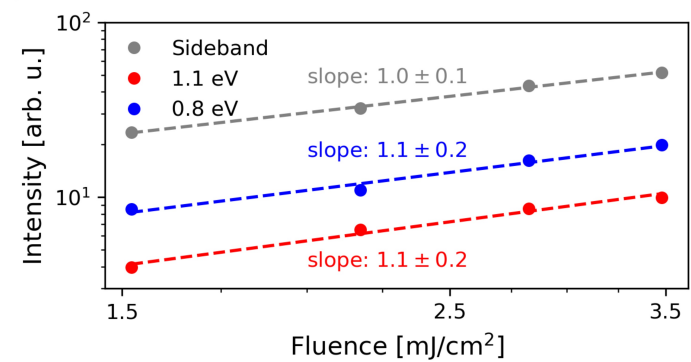

Figure 5.6.: Fluence dependence of the charge carrier occupation in the conduction band obtained with p-polarized pump light. (a) Excitation diagram for possible two-photon processes (crossed arrows) that would terminate at $E-\mathrm{E}_{\mathrm{F}}=0.8 \mathrm{eV}$. (b) Momentum map centred at $E-\mathrm{E}_{\mathrm{F}}=0.8 \mathrm{eV}(0.7$ $\mathrm{eV}-0.9 \mathrm{eV}$ ) showing the anisotropic population of the conduction band at $\Delta t=0 \mathrm{fs}$, as well as the contribution of the sideband (SB) centered at the $K$-point. The colored box indicates the regions of interest for the two-photon resonance. (c) Fluence dependence of the photoemission yield in the sideband (black, $0.8 \mathrm{eV}-1.4 \mathrm{eV}$ ), the two-photon resonance (blue, $0.7 \mathrm{eV}-0.9 \mathrm{eV})$, and in the area where $\mathrm{AR}$ was identified $(0.8 \mathrm{eV}$ - $1.4 \mathrm{eV})$. Taken from [80].

\subsection{Concluding remarks}

In conclusion, to the best of my knowledge, the first direct observation and analysis of Auger recombination in n-doped graphene has been shown by trMM on an ultrafast $10 \mathrm{fs}$ timescale by analyzing the energy and momentum distribution of excited charge carriers. Thereby the strong optical excitation of resulted in a macroscopic number of highly excited charge carriers at energies higher than reached by the optical excitation itself. Depending on the dominant scattering processes, a distinct temporal structure on a sub-50-fs timescale has been identified and can be used in the future for further studies on primary thermalization events in graphene (see section 7).

The estimated timescale of $\mathrm{AR}$ in this work seems to be reasonable compared to the IE process observed by Gierz et al. [200] which evolves on a similar timescale even though the different experimental parameters (doping, fluence, ...) make a quantitative comparison difficult.

Contributions from any higher order non-linear effect (2-photon absorption or higher-order sideband generation) can be safely ruled out to the observed dynmics, due to the distinct time-delays and from the fluence dependent measurement, as discussed beforehand (section 5.4.2). Moreover, the post-pulse (see section 4), which is also present in this measurement does not influence the scattering dynamics in 
the first 10th of femtoseconds, but definitely changes the non equilibrium response of the system at longer timescales, as further elucidated in the appendix (sec. C.3). For this reason, no further analysis of the long-term dynamics (i.e., the cooling of the hot Fermi-Dirac distribution) is presented within this thesis. 
$\sqrt{c \text { coued }} 6$

\section{Electromagnetic dressing of the electron energy spectrum of $\mathrm{Au}(111)$ at high momenta}

The aim of this chapter is to study the electromagnetic dressing, i.e., the sideband generation, in the full photoemission horizon of a $\mathrm{Au}(111)$ crystal to determine the contribution of Floquet-Bloch and LAPE (laser-assisted-photoelectric effect) physics to the overall sideband yield. The content of this chapter reproduces significant parts of the publication "Electromagnetic dressing of the electron energy spectrum of $\mathrm{Au}(111)$ at high momenta" [79] and includes additional evaluation and discussion. Thereby, the author contributed as follows: The author took part in planning the overall experiment and worked together with Alexander Osterkorn, Tridev A. Mishra, Salvatore R. Manmana and Stefan Kehrein for the moddeling of the data. The measurements and data analysis were performed mainly by the author together with David Schmitt. The author took part in the interpretation of the data and in writing of the manuscript.

\subsection{Introduction}

On-demand engineering of the quantum-mechanical properties of condensed matter has great potential for technological innovation. Possible control tactics, to steer a quantum material in a desired state of mater, are therefore highly soughtafter [291, 292].

One pathway is the so called "Floquet engineering", manipulating the quantum system with a periodic driving [88, 89] by, e.g., an oscillating electric field, which can be delivered using the femtosecond light pulses of modern laser-amplifier systems. By changing the electric field strength $E$, the frequency $\omega$, the pulse duration $\tau$, or the polarization of the light field, novel phases of matter can be created, which was already realized for selected examples [92, 293, 294]. 
The external light-field induces an additional time-periodicity onto the system, which results in Floquet states [90, 91], the temporal analogue to the well known Bloch states in solid state physics [295]. The eigenstates of the equilibrium system are then said to be dressed and the full light-matter-coupled system can be described by Floquet-Bloch states (compare section 2.2).

Time- and angle-resolved photoelectron spectroscopy (trARPES) is largely considered as the method of choice for the unambiguous identification of such light-dressed eigenstates [92, 175, 293].

In a trARPES experiment, an intense, long-wavelength, pump pulse spanning multiple optical cycles is used to dress the equilibrium band structure, while a second, delayed, ultraviolet (UV) or extreme ultraviolet (EUV) pulse is used to probe the induced dynamics. For a clear identification of Floquet-Bloch bands, the laserassisted photoelectric effect (LAPE) has to be considered additionally [92, 93, 294. Both processes, i.e., Floquet-Bloch bands as well as LAPE, are evident in trARPES experiments as so-called sidebands, replica features of the main photoemission lines separated by the pump laser photon energy (see figure 6.1).

However, while Floquet-Bloch bands represent a coherent modification of the initial state band structure, LAPE is a final state effect, in which the photoemitted electron interacts with the electric field of the pump pulse [94, 95, 127]. Even though LAPE has no direct effect on the material's properties it was successfully utilized to study femto- to attosecond-dynamics [69, 132 137]. Recently, the first observation of LAPE from liquid surfaces has been made [138.

A seminal work on the topological insulator $\mathrm{Bi}_{2} \mathrm{Se}_{3}$ showed that Floquet-Bloch and LAPE states can occur simultaneously, with potential scattering amplitude between both (see figure 6.1] [92, 93]. These studies focused on electronic bands located at the surface Brillouin center ( $\Gamma$-point, $k_{x, y} \approx 0 \AA^{-1}$ ), where electrons are photoemitted nearly perpendicular to the surface. This was used as an approximation in the subsequent modelling. However, in order to probe, for example, the dynamical creation of a Floquet topological insulator on graphene [125], electronic bands at the edge of the Brillouin zone have to be accessed. Using EUV light, such photoelectrons would leave the surface region under a large photoemission angle and the approximation of perpendicular emission from the surface does not hold any more.

In this chapter, the generation of sidebands at high in-plane momenta is benchmarked on the exemplary metallic surface $\mathrm{Au}(111)$. By employing a momentum microscope that maps the full momentum of all photoelectrons leaving the surface [61, 78], the formation of sidebands extending over the $1^{\text {st }}$ Brillouin zone (BZ) is investigated. From theory, based on work by Madsen et al. and others [93, 95, 129, 130], we expect a strong azimuthal modulation of the sideband intensity determined by the angle-of-incidence and polarization of the pump pulse. However, such a signature is absent in our collected photoemission data. The presented analysis shows that on metal surfaces, the sideband formation is largely determined by LAPE and further that not the impinging electric field strength of the driving light field builds up the sideband intensity, but the macroscopic screening response 


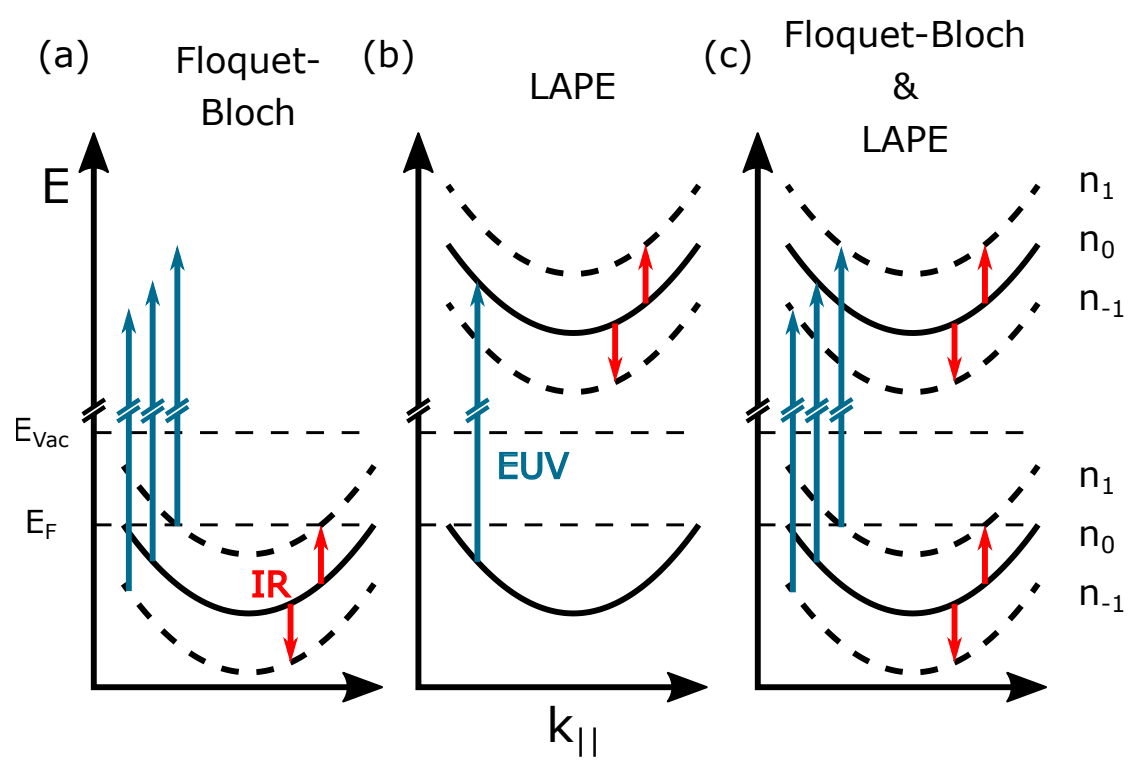

Figure 6.1.: Schematics for the electromagnetic dressing with IR light of (a) Bloch bands, yielding Floquet-Bloch bands, and (b) quasi-free electrons, leading to LAPE. In both scenarios, sidebands $\left(n_{ \pm 1}\right.$, dashed line) of the main photoemission spectral feature $\left(n_{0}\right.$, solid line) are observed in the photoemission experiment. (c) Both processes terminate at the same final state energy, requiring the consideration of scattering amplitude between both processes. Taken from [79].

of the studied material defines the electric field strength available for dressing the electromagnetic energy spectrum, which can be crucially different for Floquet and LAPE that occur within and in front of the crystal, respectively.

\subsection{Theoretical description}

The theoretical modelling for the dressed photoelectron spectrum of $\mathrm{Au}(111)$ is presented in this section, where an inital state dressing (Floquet), a final state dressing (LAPE) and scattering between both is considered (compare sec. 2.2). This work was performed in close cooperation with A. Osterkorn, T. A. Mishra, S. R. Manmana and S. Kehrein.

The theoretical description of the sideband generation follows precedent work by Madsen et al. and others [93, 95, 129, 130]. The starting point is a linear polarized driving laser field described by:

$$
\mathbf{E}=\left(\begin{array}{c}
E_{x y} \sin \left(\theta_{E}\right) \\
E_{x y} \cos \left(\theta_{E}\right) \\
E_{z}
\end{array}\right)=\left(\begin{array}{c}
E_{0} \cos \left(68^{\circ}\right) \cos (\phi) \\
E_{0} \sin (\phi) \\
E_{0} \sin \left(68^{\circ}\right) \cos (\phi)
\end{array}\right)
$$


with $\theta_{E}=\arctan \left(\frac{E_{x}}{E_{y}}\right)$, the in-plane angle of the electric field (compare figure 6.3 and $\phi$ the polarization angle $\left(\phi=0^{\circ}\left(90^{\circ}\right)\right.$ corresponds to a $\mathrm{p}(\mathrm{s})$-polarzied pump beam). The later description can be seen as the transition to the laboratory frame with an incidence angle of $68^{\circ}$ as it is used in section 6.4 .

\subsubsection{Model for the final state}

In the framework of the laser-assisted-photoelectric effect the dressing is considered in the final state in photoemission. In general, photoemission final states are free electron states in the vacuum that become distorted at the surface and are matched to high-lying Bloch waves giving rise to so-called time-inverted LEED states [296]. For metals a common simple surface model is a step potential model, the so-called "jellium" model [130]. Here, the final states are modelled as plane waves parallel to the surface and exponentially damped waves normal to the surface. The dressing by the laser field, however, can be treated in an approximate way by using the phase of free electron states dressed by a driving laser field [130, 297], which are known as Volkov waves [128, 129]. Also plain Volkov waves have proven to be useful as final states for analytical photoemission calculations [93]. Hence, the model for the final state is

$$
\phi_{V}(\mathbf{r}, t)=\frac{\mathrm{e}^{i \mathbf{k}_{x y} \cdot \mathbf{r}_{x y}}}{2 \pi} \phi_{k_{z}}(z) \mathrm{e}^{-\frac{i}{\hbar}\left(\hbar \omega_{f}+U\right) t} \sum_{n=-\infty}^{\infty} \mathrm{e}^{-i n \omega_{I R} t} J_{n}\left(\alpha, \frac{U}{2 \hbar \omega_{I R}}\right),
$$

where $\phi_{k_{z}}(z)$ is the surface-normal part of the wave function, $\hbar \omega_{f}(\mathbf{k})$ is the eigenenergy of the state. $U_{P}=\frac{e^{2} E_{0}^{2}}{4 m_{e} \omega_{I R}^{2}}$ is the ponderomotive potential and $J_{n}$ are generalized Bessel functions. The LAPE parameter $\alpha$ is described by:

$$
\alpha=\frac{e}{m_{e} \omega_{I R}^{2}} \mathbf{E} \cdot \mathbf{k}
$$

where $\mathbf{k}$ is the momentum in the final state.

\subsubsection{Model for the initial state}

The initial state in photoemission is generically a complicated Bloch wave. However, in the case of the sp band transition in Au the in-plane dispersion is well approximated as parabolic $E_{\mathrm{SP}}\left(\mathbf{k}_{\mathrm{xy}}\right)=-E_{0}^{\mathrm{SP}}+\frac{\hbar^{2}}{2 m_{e}} \mathbf{k}_{\mathrm{xy}}^{2}$ when measured with respect to $E_{\mathrm{Fermi}}$. This is in the spirit of the free electron approximation for noble metals [295], which is a common simple but often quite predictive approximation for many quantities. Note that $\mathbf{k}_{x y}$ of the inital and final states coincide due to conservation of in-plane momentum in the photoemission process.

A realistic modelling of the perpendicular momentum dispersion is more complicated. Estimating the initial state $k_{z}^{\text {in }}$ from the photoemission energies reveals that for a probing photon energy of $26.5 \mathrm{eV}, k_{z}^{\text {in }}$ is typically close to the bulk $\Gamma$ point where the dispersion is flatter than at high momenta, i.e., close to the $\mathrm{L}$ point 
(see band structure calculation and photon energy dependent photoemission data in Ref. [298] and [299]). For flat dispersions, a strong suppression of the sideband generation is expected since the dressing field couples to the momentum-dependent part of the dispersion. This is consistent with the picture of a parabolic dispersion with high effective mass in that direction.

Furthermore, the initial state dressing is subject to screening, which is further outlined in section 6.5.1. Therefore, the dressing is modelled as due to an averaged damped electric field $\mathbf{E}^{\text {in }}$ because of screening inside the metal as a first approximation. Hence, a wave function similar to Eq. (6.2) is used but with a Floquet parameter:

$$
\beta=\frac{e}{m_{e} \omega_{I R}^{2}} \mathbf{E}_{x y}^{\text {in }} \cdot \mathbf{k}_{x y}+\beta_{z}\left(E_{z}^{\mathrm{IR}, \text { in }}, k_{z}^{\mathrm{in}}\right)
$$

instead of the LAPE parameter $\alpha$.

The occupied part of the Shockley surface state (SS) has a two-dimensional parabolic dispersion with an effective mass $m_{\mathrm{SS}}^{*} \approx 0.26 m_{e}[300]: E_{\mathrm{SS}}\left(\mathbf{k}_{x y}\right)=-E_{0}^{\mathrm{SS}}+$ $\frac{\hbar^{2}}{2 m_{\mathrm{SS}}^{*}} \mathbf{k}_{x y}{ }^{2}$. At the same level of modelling as above one can hence use a Floquet parameter similar to (6.4) but with $m_{e} \rightarrow m_{\mathrm{SS}}^{*}$ and possibly a different average electric field.

\subsubsection{Photoemission amplitude}

The transition in photoemission from the initial state to the final state is calculated within first order time-dependent perturbation theory (Born approximation [93, 129]) employing a scattering matrix description. The transition amplitude from an initial state $\phi_{i}$ to a final state $\phi_{f}$ reads:

$$
\left(S^{B}-1\right)_{f i}=-\frac{i}{\hbar} \int_{-\infty}^{\infty} d t\left\langle\phi_{f}\left|\mathbf{A}_{\mathrm{EUV}} \cdot \hat{p}\right| \phi_{i}\right\rangle
$$

with the vector potential of the EUV probe $\mathbf{A}_{\mathrm{EUV}}=\frac{\mathbf{A}_{\mathbf{0}}}{2} \exp \left(-i \omega_{\mathrm{EUV}} t\right)$, where dipole and the rotating wave approximation were used. In this experiment, a driving laser fluence of $F=5 \mathrm{~mJ} / \mathrm{cm}^{2}$ is used with a pulse duration of $\tau=37 \mathrm{fs}$, resulting in an incident electric field amplitude of $E_{0}=\sqrt{\frac{2}{c \epsilon_{0}} \frac{F}{\tau}} \approx 1 \cdot 10^{9} \mathrm{~V} / \mathrm{m}$. The ponderomotive potential $U_{P}=\frac{e^{2} E_{0}^{2}}{4 m_{e} w_{I R}^{2}}$ is then of the order of $10 \mathrm{meV}$ and the term $\frac{U_{P}}{2 \hbar \omega_{I R}} \approx 0$ and can therefore be safely neglected. The generalized Bessel function reduces to the ordinary Bessel function of the first kind. 
Plugging the model for the sp wave functions into (6.5) yields

$$
\begin{aligned}
\left(S^{\mathrm{B}}-1\right)_{f i} & =-\frac{i}{\hbar} M_{f i} \sum_{m n} \int_{-\infty}^{\infty} \mathrm{d} t \mathrm{e}^{i\left(\omega_{f}(\mathbf{k})-\omega_{i}\left(\mathbf{k}^{\prime}\right)-(m-n) \omega_{\mathrm{IR}}-\omega_{\mathrm{EUV}}\right) t} J_{m}(\beta) J_{n}(\alpha) \\
& =-\frac{2 \pi i}{\hbar} M_{f i} \sum_{m n} \delta\left(\omega_{f}(\mathbf{k})-\omega_{i}\left(\mathbf{k}^{\prime}\right)-(m-n) \omega_{\mathrm{IR}}-\omega_{\mathrm{EUV}}\right) J_{m}(\beta) J_{n}(\alpha) \\
& =-\frac{2 \pi i}{\hbar} M_{f i} \sum_{m n} \delta\left(\omega_{f}(\mathbf{k})-\omega_{i}\left(\mathbf{k}^{\prime}\right)-m \omega_{\mathrm{IR}}-\omega_{\mathrm{EUV}}\right) J_{n+m}(\beta) J_{n}(\alpha)
\end{aligned}
$$

where

$$
M_{f i}=\left\langle\phi_{f}(\mathbf{r})\left|\frac{\mathbf{A}_{0}^{\mathrm{EUV}}}{2} \cdot \mathbf{p}\right| \phi_{i}(\mathbf{r})\right\rangle
$$

is the photoemission matrix element generated by the spatial parts of the wave function [130]. Within this chapter only the relative $k$-space structure of the sidebands is studied and therefore the role of the matrix element is neglected in the following. Using a Bessel function identity allows to simplify the expression for the sideband amplitudes:

$$
a_{m}:=\sum_{n} J_{n+m}(\beta) J_{n}(\alpha)=J_{m}(\beta-\alpha)
$$

The Dirac- $\delta$ in $(6.6)$ describes energy conservation during photoemission and restricts the final momentum $\mathbf{k}$ in addition to the momentum conservation parallel to the surface. The photoemission intensity of the $m$-th order sideband is:

$$
I_{m} \sim\left|a_{m}\right|^{2}=J_{m}(\beta-\alpha)^{2}=J_{m}\left(\beta_{x y}-\alpha_{x y}+\beta_{z}-\alpha_{z}\right)^{2} .
$$

Note that the Bessel function obeys: $J_{-m}(\beta-\alpha)=(-1)^{m} J_{m}(\beta-\alpha)$ for integer $m$ so that the intensity of the sidebands $I_{m}=I_{-m}$. The Bessel function $J_{1}$ can be approximated for small parameters $|\beta-\alpha| \ll \sqrt{2}$ resulting in a sideband amplitude $(m=1)$ :

$$
\left|a_{1}\right|^{2} \sim \frac{1}{4}(\beta-\alpha)^{2} .
$$

This is justified in our case with an incident electric field $E_{0}=1 \cdot 10^{9} \mathrm{~V} / \mathrm{m}$ yielding a maximum LAPE parameter of $\alpha_{\max }=1.28$ and only small corrections due to $\beta$ because of the efficient screening (compare section 6.5.1), i.e., $\beta \approx 0$. Generically, since $\beta_{x y}-\alpha_{x y}=\frac{e}{m_{e} \omega_{I R}^{2}}\left(\mathbf{E}_{x y}^{\text {in }}-\mathbf{E}_{x y}\right) \cdot \mathbf{k}_{x y}$ the sideband generation for the sp band states due to the in-plane electric fields will be suppressed if the electric field in the metal is only weakly screened. 


\subsubsection{Consequences of strong screening}

Since a strong damping of the electric fields is anticipated, which is further underpinned by a Fresnel equation estimation (see section 6.5), the special case $\beta_{x y}, \beta_{z} \rightarrow 0$ of pure LAPE is considered, leading to a sideband amplitude:

$$
\left|a_{m}\right|^{2} \sim J_{m}(\alpha)^{2}
$$

In previous works [92, 93] the in-plane component of the electric field was typically neglected if all electrons are photoemitted nearly perpendicular to the surface $\left(k_{z} \gg k_{x}, k_{y}\right)$. Here this approximation is a priori not valid, because electrons are photoemitted under large photoemission angles and thus high in-plane momenta. If the in-plane components of the electric field are taken into account, the result yields a dependence on the azimuthal angle:

$$
\left|a_{1}\right|^{2} \sim J_{m}\left(\frac{e}{m_{e} \omega_{I R}^{2}}\left(E_{x y} k_{x y} \cos \left(\theta_{k}-\theta_{E}\right)+E_{z} k_{z}\right)\right)^{2},
$$

with $\theta_{k}=\arctan \frac{k_{x}}{k_{y}}$ (see figure 6.3. The same approximate form of the Bessel function can be used as above such that the sideband amplitude for the first sideband $(m=1)$ is given by $J_{1}(\alpha)^{2} \approx \frac{\alpha^{2}}{4}$ :

$$
\left|a_{1}\right|^{2} \sim \frac{1}{4}\left(\frac{e}{m_{e} \omega_{I R}^{2}}\left(E_{x y} k_{x y} \cos \left(\theta_{k}-\theta_{E}\right)+E_{z} k_{z}\right)\right)^{2}
$$

Here, one can notice that the sideband amplitude and hence also the sideband intensity $(m=1)$ scales with $\frac{1}{\omega_{I R}^{4}}$. In general, the in-plane electric field components are conserved across the metal-vacuum interface. In the case of screening, one therefore has to reconsider equation 6.13 with $E_{x y}=0$ leading to:

$$
\left|a_{1}\right|^{2} \sim \frac{1}{4}\left(\frac{e}{m_{e} \omega_{I R}^{2}} E_{z} k_{z}\right)^{2} .
$$

In conclusion, the sideband amplitude of the first sideband $\left|a_{1}\right|^{2}$ has been derived with and without screening, i.e., $E_{x y}=0$ and $E_{x y} \neq 0$ (see figure 6.2). As one will later see in the results section 6 (6.4) the experimental data can be explained by an efficient screening of the in-plane electric field component $E_{x y} \approx 0$. A detailed discussion of the screening picture can be found at the end of this chapter in section 6.5 . 


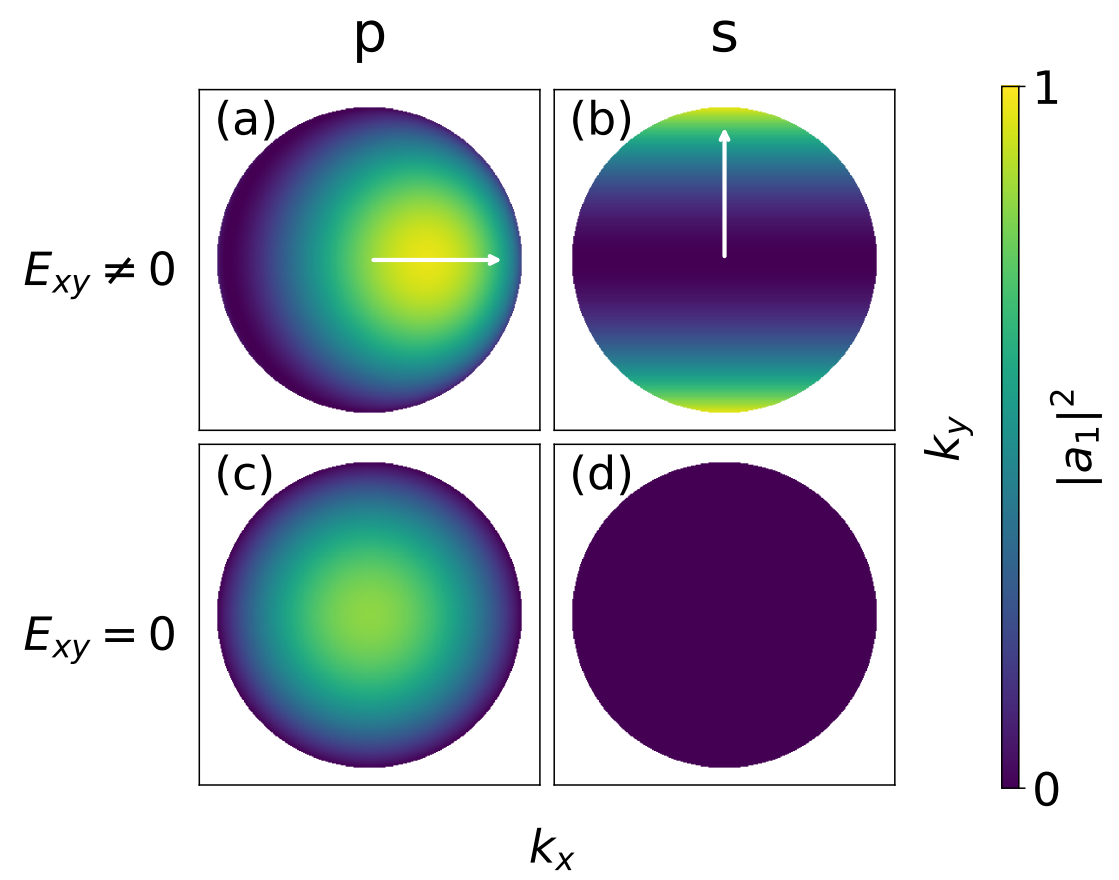

Figure 6.2.: (a,b) Calculated in-plane momentum distributions of the LAPE sideband amplitude $\left|a_{1}\right|^{2}$ after Eq. 6.12 for p- and s-polarized light impinging along $k_{x}$ in an oblique angle of incidence; the white arrow indicates the direction of the in-plane electric field component. Sideband yield can be expected for LAPE in both polarizations. (c,d) If the in-plane electric field components are screened $\left(E_{x y}=0\right)$, no sidebands are expected for s-polarized light. In p-polarized driving, $\left|a_{1}\right|^{2}$ is symmetric around the $k_{x y}=0$. Note that all plots are visualized on the same color scale, in the full accessible photoemission horizon. The calculations were performed for an incidence angle of $68^{\circ}$. Taken from [79].

\subsection{Measurement details}

The experimental setup is sketched in Fig. 6.3. A detailed description of the system was already given in chapter 4. Here, the fundamental at $1.2 \mathrm{eV}$ with a pulse duration of $\approx 37 \mathrm{fs}$ and a fluence $F$ of $5 \mathrm{~mJ} / \mathrm{cm}^{2}$ is utilized to generate the sidebands. The $11^{\text {th }}$ harmonic at $26.5 \mathrm{eV}$ with a pulse duration of $21 \mathrm{fs}$ is used to probe the light-driven bandstructure of the $\mathrm{Au}(111)$ surface. The polarization of the probe beam was fixed at p-polarization.

The measurements were performed with an extractor voltage of $12 \mathrm{kV}$ facilitating access to the full $1^{\text {st }}$ Brillouin zone of $\mathrm{Au}(111)$ while minimizing the field-emission. A drift voltage of $40 \mathrm{~V}$ results in an overall energy resolution of $\approx 200 \mathrm{meV}$ (see figure D.3). The $\mathrm{Au}(111)$ crystal was cleaned by subsequent heating and Ar-sputtering cycles up to $650 \mathrm{~K}$ prior to the measurement. All measurements have been performed at room temperature. 


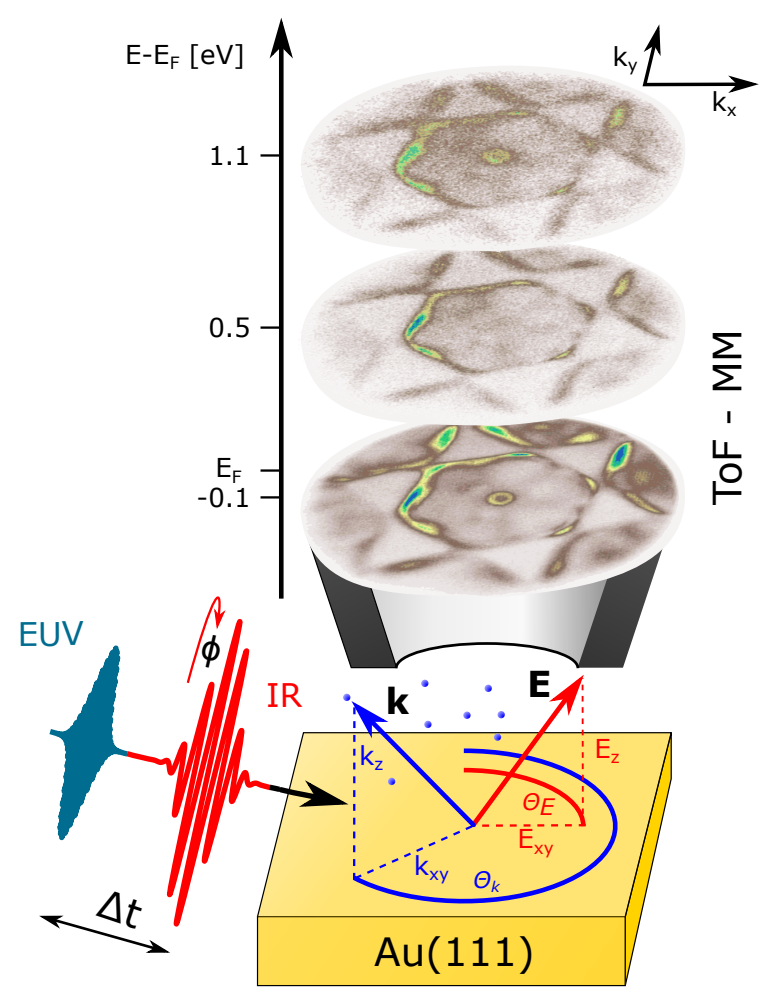

Figure 6.3.: Simplified sketch of the time-resolved momentum microscopy setup. A pump-probe approach is used to follow the the bandstructure dynamics of the $\mathrm{Au}(111)$ surface. The pump $(1.2 \mathrm{eV})$ and probe $(26.5 \mathrm{eV})$ beams have an incidence angle of $\approx 68^{\circ}$. The time-of-flight momentum microscope detects all photoemitted electrons up to photoemission angles of $\pm 90^{\circ}$ and allows to measure the whole $3 \mathrm{D}$ information $\left(k_{x}, k_{y}, E_{\text {Kin }}\right)$ within a single measurement. The angles $\theta_{E}=\arctan \left(\frac{E_{x}}{E_{y}}\right)$ and $\theta_{k}=\arctan \left(\frac{k_{x}}{k_{y}}\right)$ measure the in-plane angle of the electric field and the photoelectron momentum respectively. Taken from [79].

First, a time-resolved measurement from $\Delta t=-100$ fs to $\Delta t=+100$ fs was performed to estimate the perfect pump/probe overlap with a nearly p-polarized pump beam ${ }^{1}$ (shown in the appendix figure D.2). Subsequently, the delay was fixed at $\Delta t=0 \mathrm{fs}$ and the polarization of the pump beam was rotated in $10^{\circ}$ steps and the driven ARPES spectra were recorded. The integration time for each polarization step was $2 \mathrm{~h}$. Details on the raw-data post processing, e.g., the correction for lense errors and the energy calibration can be found in the appendix $\mathrm{D}$.

\footnotetext{
1 The polarization of the pump beam was not perfectly adjusted at the start of the experiment $\left(\phi \approx 10^{\circ}\right.$; compare $\left.6.4(\mathrm{~b})\right)$. In the following we refer to it as p-polarized pump.
} 


\subsection{Results}

Selected momentum maps of the three-dimensional momentum microscopy data sets are shown in figure 6.4. The main spectral features are the parabolic Shockley surface state (SS) at the $\bar{\Gamma}$ point with a binding energy of $390 \mathrm{meV}$ and the dispersing sp-bands at the edge of the first surface Brillouin zone. At higher in-plane momentum $\left(k_{x}, k_{y}\right)$ the sp-band transition in the second surface Brillouin zone is visible (fig. 6.4 (a) top row). In p-polarized driving, replica sideband structures of the SS

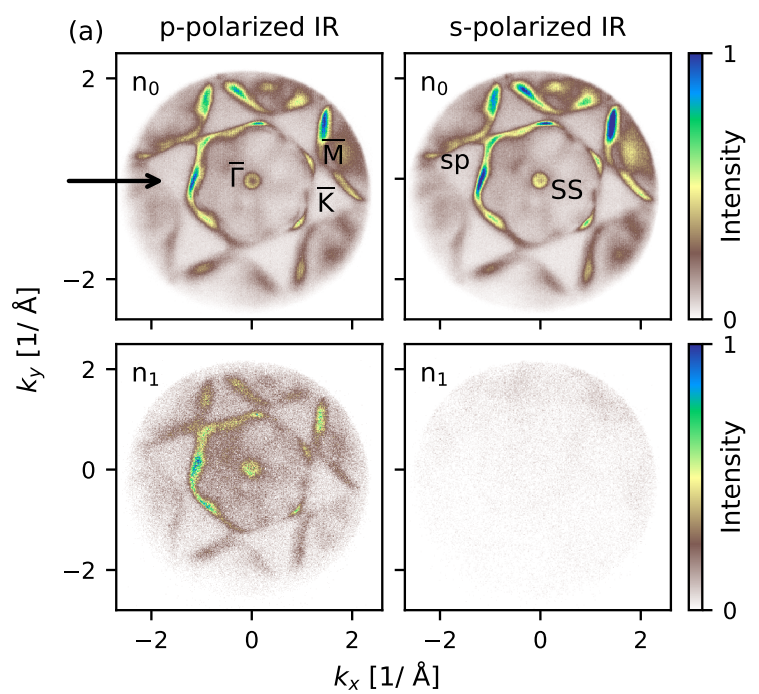

(b)

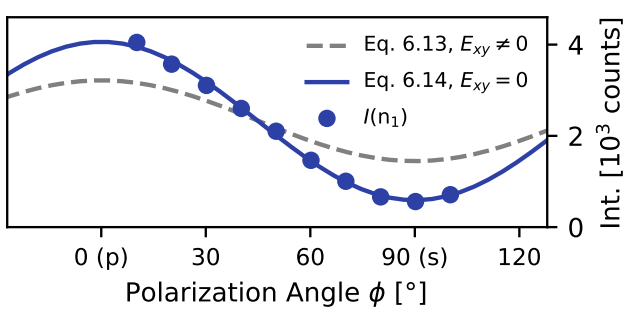

(c)

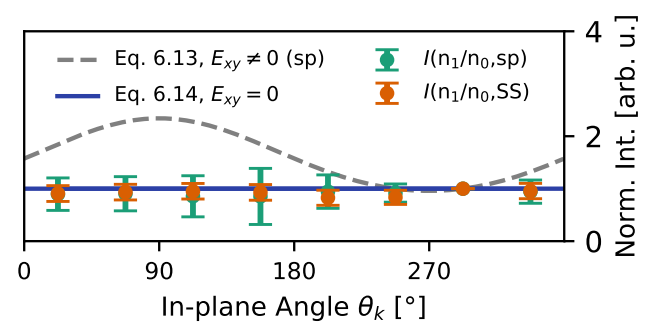

Figure 6.4.: (a) $\left(k_{x}, k_{y}\right)$-resolved momentum maps extracted from the threedimensional momentum microscopy data obtained with p-polarized EUV light and p- (left column) and s- (right column) polarized IR driving light in temporal overlap. The top row shows momentum maps taken close to the the Fermi-level that we label as the zero-photon-order sideband $n_{0}$. The high-symmetry points $\bar{\Gamma}, \overline{\mathrm{K}}, \overline{\mathrm{M}}$, and the Shockley surface band (SS) as well as the sp-band tranitions (sp) are indicated. The black arrow represents the direction of light incidence. The bottom row shows the first order sideband intensity $\left(n_{1}\right)$ around $E-E_{F}=+1.1 \mathrm{eV}$ above the Fermi-level for driving with p- (left) and s-polarized (right) IR light. (b) Systematic evaluation of the momentum integrated intensity of the first-order sideband $n_{1}$ when rotating from p- to s-polarized light. The data is well approximated with Eq. 6.14, implicating that the inplane electric field components are screened $\left(E_{x y}=0\right)$. (c) Azimuthal dependence of the sideband intensity of the SS band and the sp-band transition (details on the data handling are provided in the appendix D). The data can be fitted with Eq. 6.14, indicating that the in-plane component of the electric field is efficiently screened. Taken from [79].

band and the sp-band transition $1.2 \mathrm{eV}$ above the original structures are observed 
6.4 (a), bottom left). In the following, the sideband intensities are further evaluated throughout the full measured 3D data set.

First, the polarization angle $\phi$ of the driving laser field has been systematically varied from p- to s-polarization, keeping all other parameters fixed. The in-plane momentum-integrated photoemission yield of the first order sideband, $\mathrm{I}_{1}\left(k_{x y}, \theta_{k}, E-\right.$ $\left.E_{F} \approx+1.1 \mathrm{eV}\right)$, is shown in Fig. 6.4 (b). The intensity of the IR driving-induced sideband features drops systematically when the out-of-plane field component is reduced by rotating to overall s-polarization. The associated momentum maps for p- and s-polarized driving light are shown in Fig. 6.4 (a), bottom left and right. Strikingly, no distinct photoemission spectral features of the sidebands are resolved for s-polarized driving light within the noise level (which is slightly increased due to photoemission with residual light of a neighboring harmonic with $\hbar \omega=31.4 \mathrm{eV}$, see chapter 4 and in the appendix D). Based on the calculations shown in Fig. 6.2. this observation is unexpected at first: For $k_{x y}>2 \AA^{-1}$ and $\Theta_{k}=0^{\circ}$ and $180^{\circ}$, i.e., close to the edge of the photoemission horizon and perpendicular to the plane of incidence of the driving light, sideband intensitites should be resolved.

This observation can be understood, however, when taking screening of the IR electromagnetic field in front of the high-electron density $\mathrm{Au}(111)$ crystal into account. At the metallic surface, in-plane electric field components with driving frequencies below the plasmon frequency are reflected with near unity; the local in-plane electric field strength within the crystal and in front of the surface is close to zero as evidenced further by the Fresnel equations estimation (see section 6.5). Thus, at the metallic surface, Eq. 6.14 is valid (compare figure 6.2 bottom row). In agreement with experiment, no sideband amplitude is present in s-polarized driving. Following this reasoning, the polarization dependent photoemission yield in Fig. 6.4 (b) is fitted with Eq. 6.14, which nicely describes the experimental results (blue fit). In contrast, if the in-plane field components are included, i.e., use Eq. 6.13, the data is not described to a satisfactory level (grey dashed line).

Having identified the absence of sidebands caused by in-plane field components of the driving laser, it is insightful to study the p-polarized driving light that contains both in-plane and out-of-plane field components. Since the surface normal component of the electric field $\left(E_{z}\right)$ is not effectively screened in front of the surface, LAPE sidebands must be expected. Indeed, they are clearly observed (Fig. 6.4 (a), bottom left), and now analyzed in our experimental data to verify either the asymmetric or symmetric intensity fingerprint as shown in Fig. 6.2 (a) or 6.2 (c). Therefore, in Fig. 6.4 (c) the measured relative sideband intensity $I\left(n_{1}\right) / I\left(n_{0}\right)$ of the SS band and the sp transition is plotted as a function of azimuthal angle $\Theta_{k}$. For both cases, $I\left(n_{1}\right) / I\left(n_{0}\right)$ is not modulated with $\Theta_{k}$ (for analysis details see appendix section $\mathrm{D}$ ). For the SS band located at the $\bar{\Gamma}$-point, this is expected as $k_{x y} \approx 0.15 \AA^{-1}$ and thus negligible small; Eq. 6.13 and Eq. 6.14 would yield similar sideband intensities even with contributions from $E_{x y} \neq 0$. However, the sp band transition is probed at $k_{x y} \approx 1 \AA^{-1}$, but is still not modulated with $\theta_{k}$. Thus, as well under p-polarized driving, we can identify the screening of the in-plane electric field components as they do not contribute to the electromagnetic dressing of the energy spectrum of 
the metallic surface at high momenta.

As an additional test of the screening model, one can check for the $k_{z}$ dependence that is expected from equation 6.14. A similar analysis is performed as done in Fig. 4 (c) and the momentum maps are divided into areas 1 to 4 as shown in Fig. 6.5 (note that here the full $\theta_{k}$-range is integrated). As discussed in the appendix in section $\mathrm{D}$, the contribution of the $13^{\text {th }}$ harmonic is corrected in the integration process; region 1, that contains photoemission from the surface band is used as a reference. From the value of the in-plane momentum $k_{x y}$ centered in the different areas 1 to 4 , the $k_{z}$ momentum is calculated in the free electron picture to

$$
k_{z}=\sqrt{\frac{2 m E_{k i n}}{\hbar^{2}}-\left(k_{x y}^{2}\right)},
$$

and the normalized intensity is plotted as a function of $k_{z}$ momentum (see Fig. 6.5). As expected from Eq. 6.14 the intensity can be described by a $k_{z}^{2}$ dependence, which is in accordance to the screening picture.

Further insight can be gained from pictorial comparison of the momentum distribu-

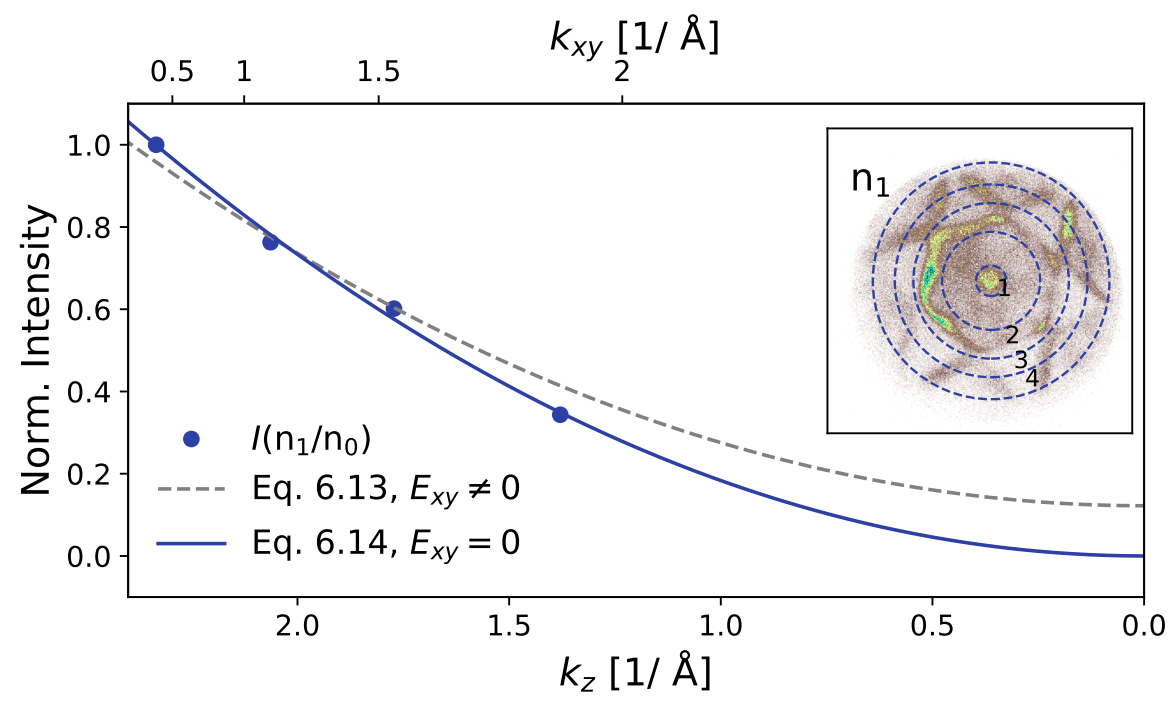

Figure 6.5.: $k_{z}$-dependence of the sideband intensity of the SS band, the sp transition, and the outer part of the second surface Brillouin zone in ppolarized driving, as indicated by the areas 1 to 4 in the inset. When considering screening of the in-plane electric field components, the intensity follows a $k_{z}^{2}$ behavior as expected from Eq. 6.14 (blue line). In contrast, Eq. 6.13 (without screening) does not describe the data satisfactorily (grey dashed line). Taken from [79].

tion in the sideband. The expected momentum maps of the sideband are generated by taking the zero-order sideband $\mathrm{n}_{0}$ in the undriven case as a reference and subsequently the sidebands $\mathrm{n}_{1}$ are calculated with (equation 6.14. $E_{x y}=0$ ) and without 
screening (equation 6.13, $E_{x y} \neq 0$ ) of the in-plane electric field (see Fig. 6.6). A comparison between the calculated momentum maps and the measured momentum map of the sideband $n_{1}$ agrees well for the screened case, whereas one can notice substantial differences between the unscreened calculated sideband and the measured one (as highlighted by the black arrows).

In this section two major conclusions have been identified:

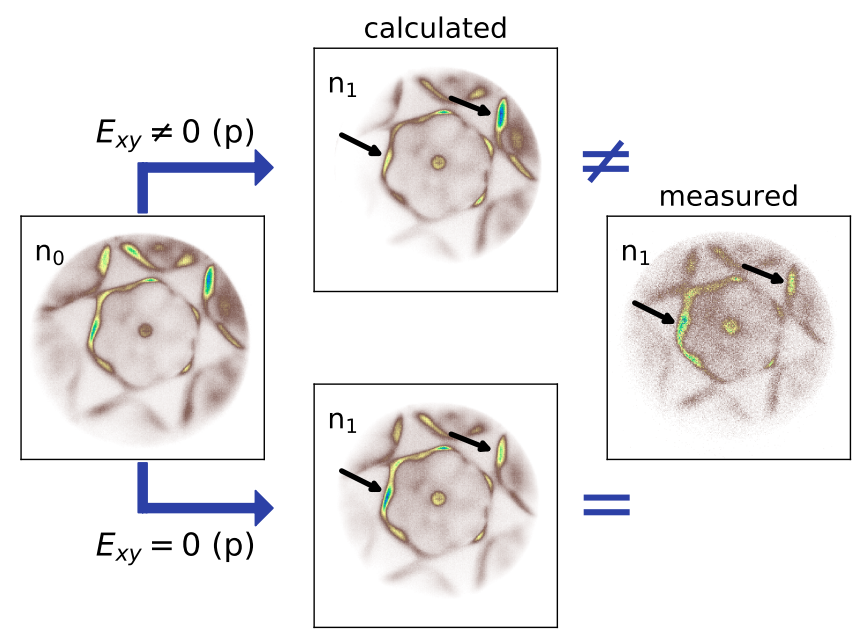

Figure 6.6.: Pictorial illustration of the sideband momentum distribution with $\left(E_{x y}=0\right)$ and without $\left(E_{x y} \neq 0\right)$ screening. From left to right, the zero-order sideband in s-polarized driving, the calculated first order sidebands with (bottom) and without (top) screening, and the measured first-order sideband in p-polarized driving are shown. The black arrows mark characteristic points of the momentum distributions.

(a) Driving with s-polarized light should create LAPE sidebands with increasing intensity towards the photoemission horizon [see Fig. 6.2 (b)].

(b) If the studied material system is highly reflective for the applied driving frequency, like the $\mathrm{Au}(111)$ crystal for IR light, the in-plane electric field components are effectively screened in the bulk and in front of the surface; only surface normal field components can lead to the formation of sidebands.

The determination of the local electric fields in the Frensel picture (i.e., the screened electric fields) and possible contributions from Floquet-Bloch states will be elucidated in the following section 6.5 with emphasis on the role of screening in the framework of Floquet engineering. 


\subsection{Discussion}

\subsubsection{Calculation of the screened electric fields using Fresnel equations}

In a simple model an abrupt metal-vacuum interface is used that is described by Frensel equations with the dieletric function for $\mathrm{Au}$ 301 $\left(\epsilon_{1}=-44.252, \epsilon_{2}=\right.$ 2.0375). The orientation of the axis are defined so that the plane of incidence is the xz-plane and the surface of the metal is the xy plane. The electric fields along $\mathrm{x}-$ and y-direction, corresponding to an p- and s- polarized incident electric field, can then be written to [302, 303]:

$$
\begin{aligned}
& \frac{E_{x}^{2}}{E_{0}^{2}}=\frac{4 \cos \left(\theta_{d}\right)^{2} S^{1 / 2} \epsilon_{d}}{\left(\epsilon_{1}^{2}+\epsilon_{2}^{2}\right) \cos \left(\theta_{d}\right)^{2}}, \\
& +S^{1 / 2} \epsilon_{d} \sqrt{2} \cos \left(\theta_{d}\right) u \epsilon_{d}^{1 / 2}\left(S^{1 / 2}+\epsilon_{d} \sin \left(\theta_{d}\right)^{2}\right) \\
& \frac{E_{y}^{2}}{E_{0}^{2}}=\frac{4 \cos \left(\theta_{d}\right)^{2} \epsilon_{d}}{\left.\epsilon_{d} \cos \left(\theta_{d}\right)^{2}+S^{1 / 2}+\sqrt{2} \cos \left(\theta_{d}\right) u \epsilon_{d}^{1 / 2}\right)},
\end{aligned}
$$

with the incident electric field amplitude $E_{0}$, the angle of incidence $\theta_{d}$, the vacuum dielectric constant $\epsilon_{d}$ and

$$
\begin{gathered}
S=\left(\epsilon_{1}-\epsilon_{d} \sin \left(\theta_{d}\right)^{2}+\epsilon_{2}^{2}\right), \\
u=\left(S^{1 / 2}+\left(\epsilon_{1}-\epsilon_{d} \sin \left(\theta_{d}\right)\right)^{1 / 2} .\right.
\end{gathered}
$$

The electric field in z-direction can be calculated below the metal-vacuum interface $E_{z_{-}}$or above $E_{z_{+}}$:

$$
\begin{gathered}
\frac{E_{z_{+}}^{2}}{E_{0}^{2}}=\frac{4 \sin \left(\theta_{d}\right)^{2} \cos \left(\theta_{d}\right)^{2}\left(\epsilon_{1}^{2}+\epsilon_{2}^{2}\right)}{\left(\begin{array}{c}
\left.\epsilon_{1}^{2}+\epsilon_{2}^{2}\right) \cos \left(\theta_{d}\right)^{2}+S^{1 / 2} \epsilon_{d} \\
+\sqrt{2} \cos \left(\theta_{d}\right) u \epsilon_{d}^{1 / 2}\left(S^{1 / 2}+\epsilon_{d} \sin \left(\theta_{d}\right)^{2}\right)
\end{array}\right.}, \\
\frac{E_{z_{-}}^{2}}{E_{0}^{2}}=\frac{4 \sin \left(\theta_{d}\right)^{2} \cos \left(\theta_{d}\right)^{2}}{\left(\begin{array}{c}
\left.\epsilon_{1}^{2}+\epsilon_{2}^{2}\right) \cos \left(\theta_{d}\right)^{2}+S^{1 / 2} \epsilon_{d} \\
+\sqrt{2} \cos \left(\theta_{d}\right) u \epsilon_{d}^{1 / 2}\left(S^{1 / 2}+\epsilon_{d} \sin \left(\theta_{d}\right)^{2}\right)
\end{array}\right.} .
\end{gathered}
$$

Since the sideband amplitude scales with $E^{2}$ it is insightful to compare the squared electric fields below the surface $\left(E_{z_{-}}^{2}\right)$ and in-plane $\left(E_{x}^{2}, E_{y}^{2}\right)$ to it's value above the surface $\left(E_{z_{+}}^{2}\right)$. From these equations above one can determine that the squared electric field drops to $\approx 0.05 \%$ inside of the metal $E_{z_{-}}^{2}$ in comparison to it's value above the interface $E_{z_{+}}^{2}$ using an angle of incidence of $68^{\circ}$, i.e., the electric field inside the metal is nearly perfectly screened as expected. For the in-plane component in the p-polarized case one can derive that $E_{x}^{2} / E_{z_{+}}^{2} \approx 1.74 \%$, so that also here the outof plane component clearly dominates. In the s-polarized case, the whole electric 
field lies in-plane but is still effectively screened in the Fresnel picture, so that $E_{y}^{2} / E_{0}^{2} \approx 1.35 \%$. Here, it is compared to the incident electric field $E_{0}$ since no outof-plane component exists $\left(E_{z_{+}}=E_{z_{-}}=0\right)$. In total, the electric field and therefore also the dressing is dominated by the electric field above the interface $\left(E_{z_{+}}\right)$.

\subsubsection{Estimation of the relative contribution of LAPE and Floquet-Bloch states to the sideband yield}

The open question is whether Floquet-Bloch contributions can be identified to the measured sideband yield in the two-color photoemission data obtained on $\mathrm{Au}(111)$. Therefore, it is insightful to look at the calculated $\beta$ (compare eq. 6.4) and thus the expected momentum fingerprint of the Floquet sideband amplitude. In the theoretical description, the form of $\beta$ has been approximated, which depends on the initial state momentum dispersion, for the two-dimensional, parabolic surface band (the SS band) and a bulk band transition with a more complex dispersion relation (the sp band transition). Therefore, in the case of perfectly parabolic bands, interference between Floquet-Bloch and LAPE bands can induce complete destructive interference, i.e., no sidebands would be observable in photoemission.

However, in the case of $\mathrm{Au}(111)$, the Floquet contribution is in any case negligible, and the measured photoemission yield of the sidebands in Fig. 6.4 (a) is caused mainly by LAPE electrons, as can be understood by considering screening of the driving electromagnetic field at the metallic surface. First, when considering the inplane field components, like discussed above, no Floquet-Bloch sideband amplitude can be expected as $E_{x y} \approx 0$ due to screening. Importantly, this statement is independent of the explicit form of the $\beta$ parameter, and thus true for all initial states, independent on their momentum dispersion. Second, for electromagnetic dressing with the out-of-plane field component $\left(E_{z}\right)$, the situation is slightly more complex. Both, in front of the surface and in the bulk material, the electric field can couple to the $k_{z}$ component of the initial (Floquet) and the final state (LAPE); the relative strength of the Floquet contribution will then depend on the explicit dispersion of the initial state. Here, the contribution of the initial state dispersion is neglected and only the relative strength of $E_{z}$ is estimated as follows: LAPE is considered to occur close to the crystal, where the surface can act as a sink for momentum conservation in the light-dressing process (i.e., $\left.E_{z_{+}}\right)$. In contrast, Floquet-Bloch bands would be created within the bulk material; the electric field has to penetrate into the crystal $\left(E_{z_{-}}\right)$. In such a simple approach, considering an abrupt metal-vacuum interface that could be described via Fresnel equations, the surface normal field component discontinuously drops at the surface barrier. From the estimation above (section 6.5.1), one already knows that the dressing is dictated by the electric field above the interface $\left(E_{z_{+}}\right)$, which clearly illustrates that the measured sideband yield is dominated by LAPE physics. In addition, it exemplifies how critical the screening capabilities of the material have to be considered, if photoemission band mapping is the method of choice for the investigation and identification of light-engineered 
electronic band structures. Even though, a light-induced coherent manipulation of the electronic band structure from a metal surface is possible and also has recently been observed using interferometric photoemission techniques [304].

\subsubsection{Screening beyond the Fresnel picture}

One has to note that the Fresnel equations might not properly describe the electric fields here even though they are widely used in ARPES experiments [305 307] to describe the electric field interacting with a system. It was pointed out early by Feibelman [308 310] that this treatment of the electric field close to the surface of a metal is insufficient and experimental deviations were observed [311]. Even today, it is still questionable how accurate Fresnel equations describe the electric field close to a metal surface. In a recent RABBIT experiment on $\mathrm{Cu}(111)$ [312], it was concluded that the macroscopic Fresnel equations can still properly describe the experiment on atomic length- and attosecond timescale, whereas a different result was obtained in an attosecond streaking experiment [136], where good agreement with theory was found employing an exponentially damped electric field at the surface (arguing that the Fresnel equations cannot be applied).

Considering in detail the streaking experiment by Neppl et al. one can note that also there the electric field vanishes quickly inside of the metallic surface, this is shown in figure 6.7. They studied the dynamics of photoelectrons emitted from a W(110) crystal with varying Mg-overlayers on top using 450 as XUV pulses (118 $\mathrm{eV}$ ) and a NIR driver (500 nm $-1000 \mathrm{~nm},<5 \mathrm{fs})$. The spatial variance of the electric field (red) predicted by time-dependent density-functional theory (TDDFT) is still reasonably close to step-function behaviour as it is expected for the Fresnel equations. This decay of the electric field needs to be connected to the probed region, which is related to the mean free path of the emitted electrons (the universal curve; see figure 2.3 . In our case this would results in $\lambda(\approx 20 \mathrm{eV}) \approx 10 \AA$, thus the Fresnel equations seem to be still a sufficient approximation ${ }^{2}$. A better understanding of the electric field close to the surface can be gained by, e.g., time-dependent density functional theory like in [136, 313].

Another way to estimate the driving electric field has been shown in [314], where it was directly reconstructed from the streaking of the photoelectrons. This experimental approach can not be easily transferred to our setun $\mathrm{b}^{3}$ but is still an interesting experiment to conduct.

\footnotetext{
${ }^{2}$ In the work by Neppl et al. the kinetic energy of the photoelectrons, in the region of interest, ranged from $\approx 70-110 \mathrm{eV}$ close to the minimum of the mean-free path. Therefore, they likely needed to consider the spatial variance of the electric field. ${ }^{3}$ In this work they streaked photoelectrons using a THz electric field on ps timescales and probed the system with $100 \mathrm{fs}$ UV pulses. In our case that would translate to an attosecond streaking experiment.
} 


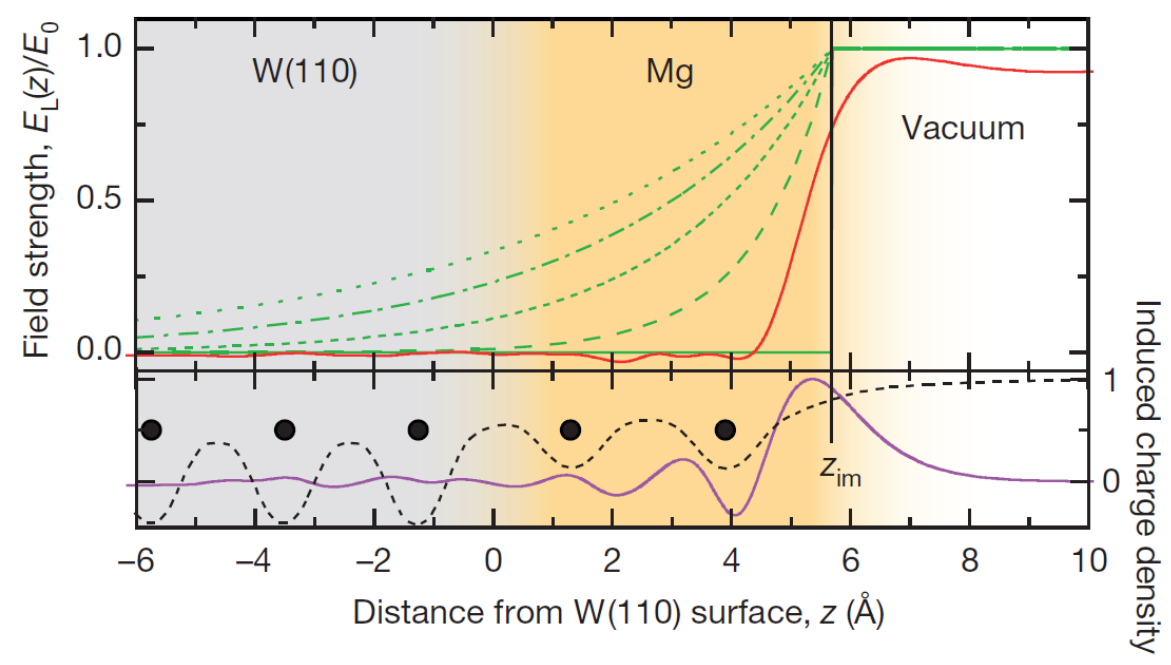

Figure 6.7.: The upper panel illustrates the different screening scenarios for the outof-plane component $E_{L}(z, t)$ of the electric field, considered for the example of $2 \mathrm{Mg}$ adlayers (orange shading) on W(110) (grey shading). The green lines denote different exponential screening lengths $(0 \AA, 1.3 \AA$, $2.6 \AA, 3.9 \AA, 5.2 \AA)$. The red line is the spatial variation of $E_{L}(z, t)$ at the interface predicted by TDDFT. The lower panel is a snapshot of the NIR-induced charge density at the metal-vacuum interface at the maximum of the laser pulse derived by TDDFT (magenta line). The position of the dynamic image plane $z_{i m}$ is indicated as a vertical blacksolid line. The lattice potential (averaged parallel to the crystal surface) employed in the DFT calculations is shown as a dotted black line. The positions of the $\mathrm{Mg}$ and $\mathrm{W}$ atoms at the interface along the surface normal are indicated as black dots. Reprinted by permission from Springer Nature Customer Service Centre GmbH: Springer Nature, Nature, Direct observation of electron propagation and dielectric screening on the atomic length scale by S. Neppl et al. (O)(2015).

\subsubsection{Concluding remarks}

In conclusion, a systematic evaluation of the electromagnetic dressing of the electron energy spectrum at high in-plane momenta, i.e., within the full measured photoemission horizon has been presented. In contrast to photoemission experiments focusing on features close to the $\bar{\Gamma}$-point $\left(k_{x y} \approx 0 \AA^{-1}\right)$ [92, 94, 95], for $k_{x y}$ near the photoemission horizon, the in-plane electric field components $E_{x y}$ can, in principle, induce light-dressing of free electron states, i.e., LAPE. However, the analysis shows that not the external electric field strength defines the dressing response, but that the local electric field strength at the crystal has to be considered. Thus, depending on the frequency dependent dielectric tensor, sideband yield can be largely suppressed (and potentially enhanced) in the two-color photoemission experiment. Our analysis further shows that the distinct separation of Floquet-Bloch and LAPE con- 
tributions in a two-color photoemission experiment is challenging. From modelling of the expected momentum fingerprints for bands with specific initial state momentum dispersions, such as done for two-dimensional linear bands in Ref. [92, 93], and carried out for parabolic bands here, further insight can be gained. Especially for the case of 3D dispersive bulk bands, further theoretical work is needed. Additionally, a more detailed theoretical study including the spatial dependence of the electric fields in comparision to the probability distributions of the involved initial states might be necessary to gain a better understanding of the role of screening. Beyond the macroscopic material properties that define the local electric field strength that can potentially build up Floquet-Bloch (and LAPE) sidebands, further theory efforts suggest that also the time scale of decoherence of the optical excitation [315 317], and the pulse duration of the driving field in relation to the optical cycle duration [318, 319] can hinder the creation of light-engineered band structures, even so sufficient electric field strength is available for efficient dressing. Based on the results gained here and those predictions, several implications follow for the on-demand creation and detection of light-engineered band structures. One has to consider the macroscopic material properties, and choose driving conditions that guarantee a minimum phase space into which energy can dissipate. 


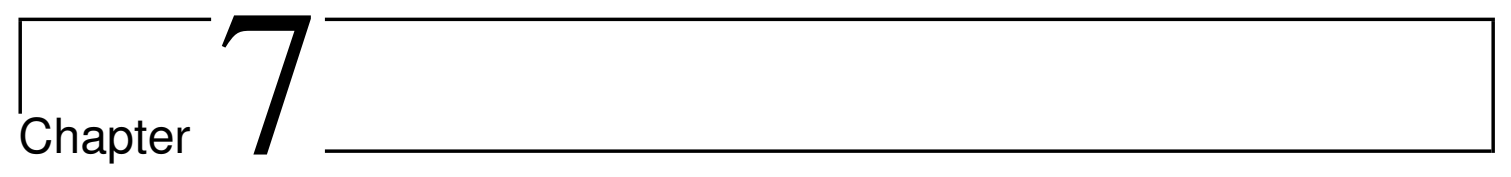

\section{Concluding remarks}

The summary and outlook given here may partially overlap with the corresponding parts in the relevant publications for this thesis, i.e., [78 80].

\subsection{Summary}

In this thesis a new generation of a time- and angle-resolved photoemission experiment has been built-up from scratch and benchmarked [78]. Additionally, first key experiments have been conducted to study the role of Auger recombination in graphene [80], and the generation of light-dressed electron energy spectra on a $\mathrm{Au}(111)$ crystal [79].

The trMM setup consists of a momentum microscope together with a lab-based high harmonic generation setup and a synchronized variable pump beam line all operating at the exceptional repetition rate of $1 \mathrm{MHz}$. It's outstanding potential lies in the acquisition of $4 \mathrm{D}$ data sets, containing the time-dependent evolution of the energy- and in-plane-momentum-resolved photoelectron distributions $\left(\mathrm{I}\left(E, k_{x}, k_{y}, \Delta t\right)\right)$ within the whole surface Brillouin zone. First, a general classification of the tr-momentum microscopy setup, built in this thesis, is performed within the current state-of-the-art of time- and angle-resolved photoemission experiments.

The first time-resolved momentum microscopy experiments have been demonstrated recently employing FEL radiation [82, 83] and visible light (400 nm) within a 2PPE experiment [84], and within this thesis (chapters 4 .5 and 60) and other works [81, 85-87]) using a lab-based high harmonic generation (HHG) source, thereby defining the current state-of-the-art in time-resolved momentum microscopy worldwide. Such setups offer new perspectives and advantages, but, due their distinct detection scheme new challenges and limitations arise as well, as discussed in section 4.5.1. The large amount of data, together with additional signal distortions from different sources, necessitates new ways of data storage, handling and correction schemes as discussed in [60, 85, 267, 269] and section 3.3. Both hemispherical detectors and momentum microscopes have their distinct dis-/advantages and, de- 
pending on the envisaged experiment, one of the systems is more advantageous to use, which is nicely described in [85].

In chapter 5 the ultrafast scattering dynamics in graphene have been evaluated and the process of Auger recombination could be discerned from other scattering events by employing the new momentum microscopy setup. This is an important step in designing and controlling charge carrier multiplication in graphene and possible applications thereof [4, 185, 192, 196, 320]. Here, multiple charge carriers are generated by impact excitation after an absorption of a single photon. The driving process, i.e., impact excitation, has been studied recently by trARPES on ultrafast timescales [200], whereas the counteracting process, i.e., Auger recombination, has been elusive so far. This experimental gap in the non-thermal evolution of the charge carrier dynamics in graphene has been tackled within this thesis.

The engineering by light, i.e., employing so-called "Floquet Engineering" to tailor the properties of a solid [88, 89], has been studied in chapter 6. Thereby, the application of a periodic electric field, delivered from, e.g., an ultrashort laser pulse, is used to manipulate the material's properties. By changing the electric field strength $E$, the frequency $\omega$, the pulse duration $\tau$, or the polarization of the light field, novel phases of matter can be created and the subsequent changes in the bandstructure are mapped by ARPES in a time-resolved experiment [92, 175, 293]. Here, one has to discern the dressing of the free-electron final states in photoemission (LAPE $=$ laser-assisted photoelectric effect) and the direct dressing of the bandstructure of the material (Floquet-Bloch states) [92, 93, 294]. The momentum microscopy setup has been used to measure the time-dependent dressing of the photoelectron spectrum from a $\mathrm{Au}(111)$ crystal in the whole surface Brillouin zone (SBZ) to distinguish LAPE from the generation of Floquet-Bloch states. Thereby, the screening of the electric field at the vacuum metal interface has been identified to hinder the build-up of Floquet-Bloch bands. This result, even though slightly disappointing, is still an important step towards "Floquet engineering" and can in combination with other works explain that so far light-dressed bandstructures have only been realized for selected examples [92, 293, 294, even so the first experimental confirmation has been performed in 2013. Thereby, further theoretical works suggest that the pulse duration in relation to the optical cycle duration [318, 319] and the decoherence of the excitation [315 317] can hinder the creation of Floquet-Bloch bands. 


\subsection{Outlook}

In the end, I would like to point out possibilities offered by the momentum microscopy setup, which have so far not (or only partially) been realized. Additionally a short scientific outlook for the projects in chapter 5 and 6 is given separately.

Obviously, the overall experimental setup is still relatively young and a lot of improvements can still be done. Concerning stability, it is most promising to further stabilize the laser-amplifier and the high harmonic generation scheme, which will reduce the need of post-processing of the data. In terms of energy and temporal resolution a variable driver for the HHG process is envisaged to tailor the probe pulses to the experimental needs (i.e., time resolution vs. energetic width). Furthermore, one can make use of the Fano resonance in Argon to reduce the energetic width of the EUV pulses and increase their yield if needed [321].

The momentum microscope offers a real-space mode identical to a photoemission electron microscope (PEEM), it is therefore possible to place apertures in the realspace image and thus select regions of interest down to a few $\mu \mathrm{m}$ in size. This enables the measurement of photoelectron spectroscopy data from microscopic regions on the surface of the sample [81, 322] and is commonly termed $\mu$-ARPES. Such experiments are useful for samples that are only available in microscopic sizes, such as stacked (twisted) transition metal dichalcogenides or graphene [323, 324]. First results in this direction were gained recently, where a monolayer graphene flake in the size of a few tens of $\mu \mathrm{m}$ (fabricated by the Weitz group [324, 325]), has been measured successfully employing $\mu$-ARPES with our setup.

Conversely, it is also possible to place apertures in the Fourier-plane in order to record real-space microscopy images at specific photoelectron momenta. This brings dark-field imaging capabilities to photoelectron microscopy, which can help, e.g., to clearly distinguish different regions for an inhomogeneous sample system. Additionally, the real-space imaging combined with the ToF allows energy-filtered detection of real-space images. In a pump-probe approach, such experiments might be used to observe charge transfer processes across interfaces in real-time [326] and they are able to study the creation, propagation and interference of surface plasmon polaritions and thereof resulting vortex structures [327 329].

In general, moving to longer wavelength with the driver allows to generate high harmonics with energies capable to access deeper lying core levels (compare sec. 2.3) which can further increase the information depth and can be utilized in photoelectron diffraction measurement, thereby giving access to the surface/adsorbate structures and relaxations [283, 330 333].

The creation of circular high-harmonics which was realized recently [334 336] and could offer x-ray magnetic circular dichroism (XMCD) contrast to the momentum microscope setup and subsequently allows to study magnetic domain structures, domain wall motion and skyrmions on surfaces in a time-resolved manner [337-341]. In similar fashion the x-ray magnetic linear dichroism [342] can be utilized, e.g., to study magnetocrystalline anisotropy and the switching behavior of antiferromagnetic domains [343-346]. 
Furthermore, the combination of the trMM setup with an imaging spin-filter allows for efficient and simultaneous detection of multi-dimensional spin-resolved data sets [31, 62, 280], which, e.g., will allow for spin-resolved orbital imaging. In an optical-pump - EUV-probe excitation scheme [347349], the simultaneous access to ultrafast energy-momentum-spin-resolved charge carrier dynamics is within reach.

Two-photon photoemission experiments (2PPE), as performed in [84], can also be easily transferred to our setup to study the unoccupied states above the Fermi edge. In fact, first static two-photon photoemission measurements, employing the optical parametric amplifier have already been realized together with Andi Li from the group of H. Petek (University of Pittsburgh). Here, the two-photon photemission spectra from a $\operatorname{Ag}(110)$ crystal were detected in the whole accessible $\left(E, k_{x}, k_{y}\right)$ region in a wavelength dependent manner. The analysis of the data is still ongoing and is not further presented in this thesis.

The information depth from $2 \mathrm{PPE}$ can further be increased if the experiment is performed interferometrically [304, 350, 351]. This is especially helpful in the context of light-dressed bandstructures, where the dressing dynamics might be overshadowed by the time-integrated spectra, and thus can become distinctive by probing the direct coherent response of the system [304]. An interferometric 2PPE setup will also be integrated into the lab soon.

The trMM setup proved to be beneficial for projects like orbital tomography and orbital imaging, where the photoelectron distribution $I\left(E, k_{x}, k_{y}\right)$ from an ordered absorbed molecular layer on a surface is mapped out to gain further information on molecular geometries, chemical bonds and energetic ordering of molecular states [352 355]. From the photoelectron distribution, under suitable conditions, the molecular orbitals in real space can be reconstructed with the help of phase-retrieval algorithms. The simultaneous access to $I\left(E, k_{x}, k_{y}\right)$ within the whole photoemission horizon, as offered by the trMM setup, is thereby a significant improvement compared to other works where $2 \mathrm{D}$ detectors were used, which require an additional scanning of either the momentum or energy direction.

In a first experiment, orbital tomography and orbital imaging was performed on an absorbed PTCDA monolayer in a brick wall ordering on $\mathrm{Ag}(110)$ (see figure 7.1) [77], which brings time-resolved orbital imaging in reach. Recently, time-resolved orbital tomography has been shown employing a momentum microscopy setup [87]. For details on our work the interested reader is refereed to the published manuscript [77]. 
a)

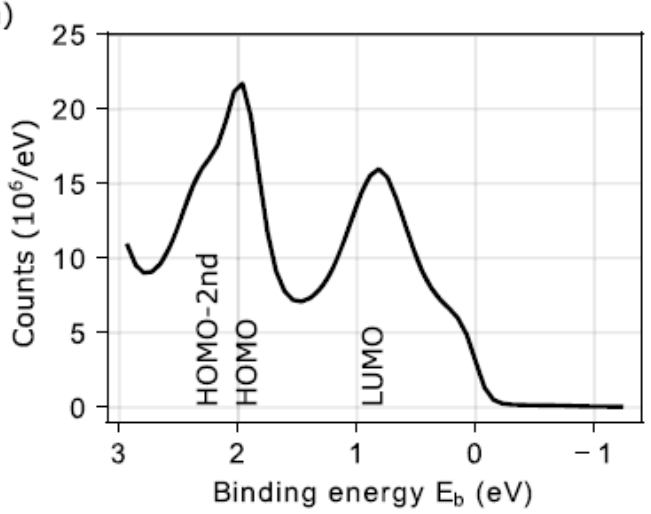

f)

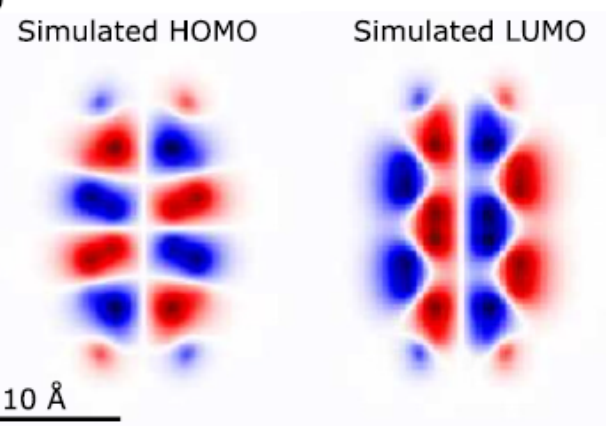

Simulated HOMO

$10 \AA$

b) $\quad A \cdot k$

c)

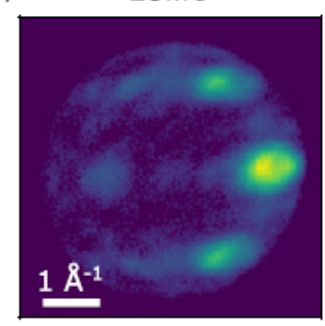

$k_{\mathrm{x}}$ d)

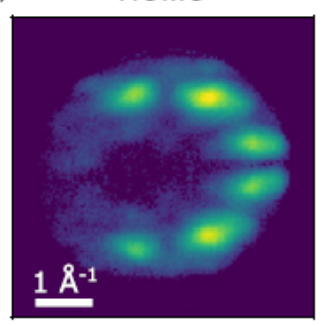

$\mathrm{k}_{\mathrm{x}}$

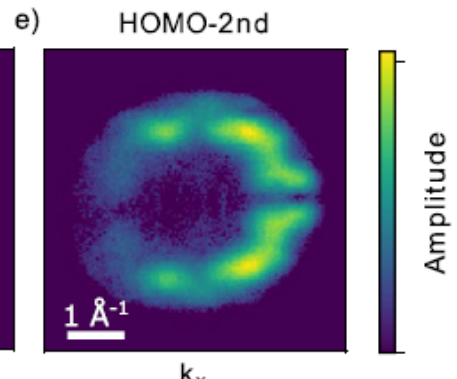

$\mathrm{k}_{\mathrm{x}}$

Figure 7.1.: Overview of the photoemission momentum microscopy measurement of PTCDA/Ag(110). a): Angle-integrated view of the photoelectron spectrum obtained after $2 \mathrm{~h}$ of measurement. b): Typical photoemission transfer function (matrix element) $|\mathrm{A} \cdot \mathrm{k}|$ for our system. c), d), e): Photoelectron momentum maps (by amplitude) of the occupied LUMO of the first monolayer, HOMO of the first monolayer and HOMO of a partial second monolayer respectively. These momentum maps were retrieved by fitting a Gaussian with fixed position and width to the photoemission spectrum measured at individual pixels. f): Simulated HOMO and LUMO orbital of PTCDA in free space as calculated using the ORCA quantumchemistry package [356]. Taken from [77]. 


\subsubsection{Ultrafast Auger recombination in graphene}

Concerning the results gained in chapter 5 , due to the in-depth access to the energyand momentum-resolved scattering dynamics, it will be highly interesting to systematically vary, for example, the doping level, fluence, excitation energy or the dielectric screening within the experiment in order to control and quantify the relative contribution of AR, IE or other scattering processes to the ultrafast charge carrier dynamics. In addition, beyond such static approaches to tailor the scattering events, dynamical control seems desirable: The pump-probe photoemission experiment has the potential to first tailor material properties, for example, via manipulation of the Coulomb interaction due to strong photodoping [36, 202, via transiently changing the electron-phonon coupling [357, or via time-periodic dressing of the electronic band structure via Floquet engineering [89], and second to probe their influence onto the scattering dynamics.

\subsubsection{Electromagnetic dressing of the electron energy spectrum of $\mathrm{Au}(\mathbf{1 1 1})$ at high momenta}

Realizing Floquet engineering seems to be a challenging task, apart from the screening behaviour which defines the available local electric field to dress the material's properties, the decoherence time of the exciation has to be considered as well [315317]. Moreover, the transient changes have to be experimentally accessible, which for trARPES is further constrained by the pump-, probe- and optical-cycle-durations to fulfil the Floquet limit (time periodicity) and thereof resulting energy resolution [318, 319]. All these restrictions point towards a smart material choice with relatively long decoherence times and small screening. Here the material class of the transition metal dichalcogenides (TMDCs) seems to be useful [358], where first observations in $\mathrm{WSe}_{2}$ point towards the successful generation of Floquet-Bloch states [175, 294]. This can be seen as a good starting point to study the beforehand discussed dependencies for Floquet engineering, which together with the analysis presented in chapter 6 allows to clearly discern Floquet-Bloch from LAPE states.

Additionally, the screening behaviour itself is still not resolved in a satisfactory manner as pointed out in section 6.5.3. Here, additional experimental and theoretical efforts are needed to clarify the local electric fields available for the dressing of different states. Especially surface or image potential states, localized at/above the surface, might show a different response due to their different spatial extend in comparison to bulk states.

Moving towards attosecond timescales would allow to further characterize the subcycle micromotions [120, 175, 359, 360], i.e., the build-up of Floquet-Bloch-states, which will be useful to gain additional insight in the beforehand discussed constraints that hinder the creation of stationary Floquet-Bloch states. 


\section{Bibliography}

[1] William Shockley and Hans J. Queisser. Detailed balance limit of efficiency of p-n junction solar cells. Journal of Applied Physics, 32(3):510-519, 1961.

[2] Cory A. Nelson, Nicholas R. Monahan, and X.-Y. Zhu. Exceeding the shockley-queisser limit in solar energy conversion. Energy \& Environmental Science, 6(12):3508-3519, 2013.

[3] Wenhao Zheng, Mischa Bonn, and Hai I. Wang. Photoconductivity multiplication in semiconducting few-layer $\mathrm{MoTe}_{2}$. Nano letters, 20(8):5807-5813, 2020 .

[4] T. Plotzing, T. Winzer, E. Malic, D. Neumaier, A. Knorr, and H. Kurz. Experimental verification of carrier multiplication in graphene. Nano letters, 14(9):5371-5375, 2014.

[5] S. Mathias, S. Eich, J. Urbancic, S. Michael, A. V. Carr, S. Emmerich, A. Stange, T. Popmintchev, T. Rohwer, M. Wiesenmayer, A. Ruffing, S. Jakobs, S. Hellmann, P. Matyba, C. Chen, L. Kipp, M. Bauer, H. C. Kapteyn, H. C. Schneider, K. Rossnagel, M. M. Murnane, and M. Aeschlimann. Self-amplified photo-induced gap quenching in a correlated electron material. Nature Communications, 7(1):12902, 2016.

[6] Nathaniel J. L. K. Davis, Marcus L. Böhm, Maxim Tabachnyk, Florencia Wisnivesky-Rocca-Rivarola, Tom C. Jellicoe, Caterina Ducati, Bruno Ehrler, and Neil C. Greenham. Multiple-exciton generation in lead selenide nanorod solar cells with external quantum efficiencies exceeding $120 \%$. Nature communications, 6(1):1-7, 2015.

[7] Matthew C. Beard, Joseph M. Luther, Octavi E. Semonin, and Arthur J. Nozik. Third generation photovoltaics based on multiple exciton generation in quantum confined semiconductors. Accounts of chemical research, 46(6):12521260, 2013.

[8] Justin B. Sambur, Thomas Novet, and B. A. Parkinson. Multiple exciton collection in a sensitized photovoltaic system. Science, 330(6000):63-66, 2010. 
[9] Markus Einzinger, Tony Wu, Julia F Kompalla, Hannah L Smith, Collin F Perkinson, Lea Nienhaus, Sarah Wieghold, Daniel N Congreve, Antoine Kahn, Moungi G Bawendi, et al. Sensitization of silicon by singlet exciton fission in tetracene. Nature, 571(7763):90-94, 2019.

[10] Bruno Ehrler, Brian J. Walker, Marcus L. Böhm, Mark W. B. Wilson, Yana Vaynzof, Richard H. Friend, and Neil C. Greenham. In situ measurement of exciton energy in hybrid singlet-fission solar cells. Nature communications, $3(1): 1-6,2012$.

[11] Luis M. Pazos-Outón, Ju Min Lee, Moritz H. Futscher, Anton Kirch, Maxim Tabachnyk, Richard H. Friend, and Bruno Ehrler. A silicon-singlet fission tandem solar cell exceeding $100 \%$ external quantum efficiency with high spectral stability. ACS energy letters, 2(2):476-480, 2017.

[12] Jonathan E. Spanier, Vladimir M. Fridkin, Andrew M. Rappe, Andrew R. Akbashev, Alessia Polemi, Yubo Qi, Zongquan Gu, Steve M. Young, Christopher J. Hawley, Dominic Imbrenda, et al. Power conversion efficiency exceeding the shockley-queisser limit in a ferroelectric insulator. Nature Photonics, 10(9):611-616, 2016.

[13] Dirk Raiser, Stephanie Mildner, Benedikt Ifland, Mohsen Sotoudeh, Peter Blöchl, Simone Techert, and Christian Jooss. Evolution of hot polaron states with a nanosecond lifetime in a manganite perovskite. Advanced energy materials, 7(12):1602174, 2017.

[14] Mingjie Li, Jianhui Fu, Qiang Xu, and Tze Chien Sum. Slow hot-carrier cooling in halide perovskites: Prospects for hot-carrier solar cells. Advanced Materials, 31(47):1802486, 2019.

[15] Zhi Guo, Yan Wan, Mengjin Yang, Jordan Snaider, Kai Zhu, and Libai Huang. Long-range hot-carrier transport in hybrid perovskites visualized by ultrafast microscopy. Science, 356(6333):59-62, 2017.

[16] H. Petek and S. Ogawa. Femtosecond time-resolved two-photon photoemission studies of electron dynamics in metals. Progress in surface science, 56(4):239310, 1997.

[17] Koichi Momma and Fujio Izumi. VESTA3 for three-dimensional visualization of crystal, volumetric and morphology data. Journal of Applied Crystallography, 44(6):1272-1276, Dec 2011.

[18] J. A. Kash, J. C. Tsang, and J. M. Hvam. Subpicosecond time-resolved raman spectroscopy of lo phonons in gaas. Physical review letters, 54(19):2151, 1985.

[19] Masayuki Yoshizawa and Makoto Kurosawa. Femtosecond time-resolved raman spectroscopy using stimulated raman scattering. Physical Review A, 61(1):013808, 1999. 
[20] Keith Moffat. Time-resolved crystallography. Acta Crystallographica Section A: Foundations of Crystallography, 54(6):833-841, 1998.

[21] Christian Rischel, Antoine Rousse, Ingo Uschmann, Pierre-Antoine Albouy, Jean-Paul Geindre, Patrick Audebert, Jean-Claude Gauthier, Eckhart Fröster, Jean-Louis Martin, and Andre Antonetti. Femtosecond time-resolved x-ray diffraction from laser-heated organic films. Nature, 390(6659):490-492, 1997.

[22] Ilya Razdolski, Alexandr Alekhin, U. Martens, D. Bürstel, D. Diesing, M. Münzenberg, Uwe Bovensiepen, and Alexey Melnikov. Analysis of the time-resolved magneto-optical kerr effect for ultrafast magnetization dynamics in ferromagnetic thin films. Journal of Physics: Condensed Matter, 29(17):174002, 2017.

[23] E. Beaurepaire, J.-C. Merle, A. Daunois, and J.-Y. Bigot. Ultrafast spin dynamics in ferromagnetic nickel. Physical review letters, 76(22):4250, 1996.

[24] Romain Geneaux, Hugo J. B. Marroux, Alexander Guggenmos, Daniel M. Neumark, and Stephen R. Leone. Transient absorption spectroscopy using high harmonic generation: a review of ultrafast x-ray dynamics in molecules and solids. Philosophical Transactions of the Royal Society A, 377(2145):20170463, 2019 .

[25] Rudi Berera, Rienk van Grondelle, and John T. M. Kennis. Ultrafast transient absorption spectroscopy: principles and application to photosynthetic systems. Photosynthesis research, 101(2-3):105-118, 2009.

[26] S. Vogelgesang, G. Storeck, J. G. Horstmann, T. Diekmann, M. Sivis, S. Schramm, K. Rossnagel, S. Schäfer, and C. Ropers. Phase ordering of charge density waves traced by ultrafast low-energy electron diffraction. $\mathrm{Na}$ ture Physics, 14(2):184-190, 2018.

[27] Max Gulde, Simon Schweda, Gero Storeck, Manisankar Maiti, Hak Ki Yu, Alec M Wodtke, Sascha Schäfer, and Claus Ropers. Ultrafast low-energy electron diffraction in transmission resolves polymer/graphene superstructure dynamics. Science, 345(6193):200-204, 2014.

[28] Armin Feist, Katharina E. Echternkamp, Jakob Schauss, Sergey V. Yalunin, Sascha Schäfer, and Claus Ropers. Quantum coherent optical phase modulation in an ultrafast transmission electron microscope. Nature, 521(7551):200 $203,2015$.

[29] Katharina E. Priebe, Christopher Rathje, Sergey V. Yalunin, Thorsten Hohage, Armin Feist, Sascha Schäfer, and Claus Ropers. Attosecond electron pulse trains and quantum state reconstruction in ultrafast transmission electron microscopy. Nature Photonics, 11(12):793-797, 2017. 
[30] Stefan Hüfner. Photolectron Spectroscopy - Principles and Applications. Springer, Berlin, 3rd edition, 2003.

[31] Shigemasa Suga and Christian Tusche. Photoelectron spectroscopy in a wide $\mathrm{h} \nu$ region from $6 \mathrm{ev}$ to $8 \mathrm{kev}$ with full momentum and spin resolution. Journal of Electron Spectroscopy and Related Phenomena, 200:119 - 142, 2015. Special Anniversary Issue: Volume 200.

[32] M. Puppin, Y. Deng, C. W. Nicholson, J. Feldl, N. B. M. Schröter, H. Vita, P. S. Kirchmann, C. Monney, L. Rettig, M. Wolf, and R. Ernstorfer. Time- and angle-resolved photoemission spectroscopy of solids in the extreme ultraviolet at $500 \mathrm{kHz}$ repetition rate. Review of Scientific Instruments, 90(2):023104, February 2019.

[33] C. M. Heyl, J. Güdde, A. L'Huillier, and U. Höfer. High-order harmonic generation with $\mu \mathrm{j}$ laser pulses at high repetition rates. Journal of Physics B: Atomic, Molecular and Optical Physics, 45(7):074020, 2012.

[34] Robert Wallauer, Johannes Reimann, Nico Armbrust, Jens Güdde, and Ulrich Höfer. Intervalley scattering in $\mathrm{MoS}_{2}$ imaged by two-photon photoemission with a high-harmonic probe. Applied Physics Letters, 109(16):162102, 2016.

[35] Steffen Eich, A. Stange, A. V. Carr, J. Urbancic, T. Popmintchev, M. Wiesenmayer, Klaus Jansen, A. Ruffing, S. Jakobs, T. Rohwer, et al. Time-and angleresolved photoemission spectroscopy with optimized high-harmonic pulses using frequency-doubled ti: Sapphire lasers. Journal of Electron Spectroscopy and Related Phenomena, 195:231-236, 2014.

[36] I. Gierz, J. C. Petersen, M. Mitrano, C. Cacho, I. C. E. Turcu, E. Springate, A. Stohr, A. Kohler, U. Starke, and A. Cavalleri. Snapshots of non-equilibrium dirac carrier distributions in graphene. Nature Materials, 12(12):1119-1124, 2013.

[37] Fang Liu, Mark E. Ziffer, Kameron R. Hansen, Jue Wang, and Xiaoyang Zhu. Direct determination of band-gap renormalization in the photoexcited monolayer $\operatorname{mos}_{2}$. Physical Review Letters, 122(24):246803, 2019.

[38] Susumu Yamamoto and Iwao Matsuda. Time-resolved photoelectron spectroscopies using synchrotron radiation: past, present, and future. Journal of the Physical Society of Japan, 82(2):021003, 2013.

[39] S. Hellmann, C. Sohrt, M. Beye, T. Rohwer, F. Sorgenfrei, M. MarczynskiBühlow, M. Kalläne, H. Redlin, Franz Hennies, M. Bauer, et al. Timeresolved x-ray photoelectron spectroscopy at flash. New Journal of Physics, 14(1):013062, 2012. 
[40] Sven Aeschlimann, Antonio Rossi, Mariana Chávez-Cervantes, Razvan Krause, Benito Arnoldi, Benjamin Stadtmüller, Martin Aeschlimann, Stiven Forti, Filippo Fabbri, Camilla Coletti, et al. Direct evidence for efficient ultrafast charge separation in epitaxial $\mathrm{WS}_{2}$ /graphene heterostructures. Science Advances, 6(20):eaay0761, 2020.

[41] J. C. Petersen, S. Kaiser, N. Dean, A. Simoncig, H. Y. Liu, A. L. Cavalieri, C. Cacho, I. C. E. Turcu, E. Springate, F. Frassetto, L. Poletto, S. S. Dhesi, H. Berger, and A. Cavalleri. Clocking the Melting Transition of Charge and Lattice Order in $1 T-\mathrm{TaS}_{2}$ with Ultrafast Extreme-Ultraviolet Angle-Resolved Photoemission Spectroscopy. Physical Review Letters, 107(17):177402, 2011.

[42] G. Rohde, A. Hendel, A. Stange, K. Hanff, L.-P. Oloff, L. X. Yang, K. Rossnagel, and M. Bauer. Time-resolved arpes with sub-15 fs temporal and near fourier-limited spectral resolution. Review of Scientific Instruments, 87(10):103102, 2016.

[43] Jan Heye Buss, He Wang, Yiming Xu, Julian Maklar, Frederic Joucken, Lingkun Zeng, Sebastian Stoll, Chris Jozwiak, John Pepper, Yi-De Chuang, et al. A setup for extreme-ultraviolet ultrafast angle-resolved photoelectron spectroscopy at 50-khz repetition rate. Review of Scientific Instruments, 90(2):023105, 2019.

[44] S. Eich, A. Stange, A.V. Carr, J. Urbancic, T. Popmintchev, M. Wiesenmayer, K. Jansen, A. Ruffing, S. Jakobs, T. Rohwer, S. Hellmann, C. Chen, P. Matyba, L. Kipp, K. Rossnagel, M. Bauer, M.M. Murnane, H.C. Kapteyn, S. Mathias, and M. Aeschlimann. Time- and angle-resolved photoemission spectroscopy with optimized high-harmonic pulses using frequency-doubled ti:sapphire lasers. Journal of Electron Spectroscopy and Related Phenomena, 195:231 - 236, 2014.

[45] S. Mathias, L. Miaja-Avila, M. M. Murnane, H. Kapteyn, M. Aeschlimann, and M. Bauer. Angle-resolved photoemission spectroscopy with a femtosecond high harmonic light source using a two-dimensional imaging electron analyzer. Review of Scientific Instruments, 78(8):083105, 2007.

[46] Edbert J. Sie, Timm Rohwer, Changmin Lee, and Nuh Gedik. Time-resolved xuv arpes with tunable 24-33 ev laser pulses at 30 mev resolution. Nature communications, 10(1):1-11, 2019.

[47] Patrick S. Kirchmann, Laurenz Rettig, Dhananjay Nandi, Uwe Lipowski, Martin Wolf, and Uwe Bovensiepen. A time-of-flight spectrometer for angleresolved detection of low energy electrons in two dimensions. Applied Physics A, 91(2):211-217, 2008.

[48] Ruslan Ovsyannikov, P. Karlsson, M. Lundqvist, C. Lupulescu, W. Eberhardt, Alexander Föhlisch, Svante Svensson, and Nils Mårtensson. Principles and 
operation of a new type of electron spectrometer-artof. Journal of Electron Spectroscopy and Related Phenomena, 191:92-103, 2013.

[49] Michael Huth, Cheng-Tien Chiang, Andreas Trützschler, Frank O. Schumann, Jürgen Kirschner, and Wolf Widdra. Electron pair emission detected by time-of-flight spectrometers: Recent progress. Applied Physics Letters, 104(6):061602, 2014.

[50] Cheng-Tien Chiang, Andreas Trützschler, Michael Huth, Robin Kamrla, Frank O. Schumann, and Wolf Widdra. Laser-based double photoemission spectroscopy at surfaces. Progress in Surface Science, page 100572, 2020.

[51] Christian Tusche, Alexander Krasyuk, and Jürgen Kirschner. Spin resolved bandstructure imaging with a high resolution momentum microscope. Ultramicroscopy, 159:520-529, 2015.

[52] B. Krömker, M. Escher, D. Funnemann, D. Hartung, H. Engelhard, and J. Kirschner. Development of a momentum microscope for time resolved band structure imaging. Review of Scientific Instruments, 79(5):053702, 2008.

[53] Fumihiko Matsui, Seiji Makita, Hiroyuki Matsuda, Takayuki Yano, Eiken Nakamura, Kiyohisa Tanaka, Shigemasa Suga, and Kera Satoshi. Photoelectron momentum microscope at BL6U of UVSOR - III synchrotron. Japanese Journal of Applied Physics, 2020.

[54] M. Patt, C. Wiemann, N. Weber, M. Escher, A. Gloskovskii, W. Drube, M. Merkel, and C. M. Schneider. Bulk sensitive hard x-ray photoemission electron microscopy. Review of scientific instruments, 85(11):113704, 2014.

[55] Christian Tusche, Martin Ellguth, Vitaliy Feyer, Alexander Krasyuk, Carsten Wiemann, Jürgen Henk, Claus M. Schneider, and Jürgen Kirschner. Nonlocal electron correlations in an itinerant ferromagnet. Nature communications, $9(1): 1-8,2018$.

[56] Aimo Winkelmann, Martin Ellguth, Christian Tusche, A. Akin Ünal, Jürgen Henk, and Jürgen Kirschner. Momentum-resolved photoelectron interference in crystal surface barrier scattering. Physical Review B, 86(8):085427, 2012.

[57] Aimo Winkelmann, Christian Tusche, A. Akin Ünal, Martin Ellguth, Jürgen Henk, and Jürgen Kirschner. Analysis of the electronic structure of copper via two-dimensional photoelectron momentum distribution patterns. New Journal of Physics, 14(4):043009, 2012.

[58] Christian Tusche, Ying-Jiun Chen, Claus M. Schneider, and Jürgen Kirschner. Imaging properties of hemispherical electrostatic energy analyzers for high resolution momentum microscopy. Ultramicroscopy, 206:112815, 2019. 
[59] S. Beaulieu, J. Schusser, S. Dong, M. Schüler, T. Pincelli, M. Dendzik, J. Maklar, A. Neef, H. Ebert, K. Hricovini, M. Wolf, J. Braun, L. Rettig, J. Minár, and R. Ernstorfer. Revealing hidden orbital pseudospin texture with timereversal dichroism in photoelectron angular distributions. Phys. Rev. Lett., 125:216404, Nov 2020.

[60] Rui Patrick Xian, Vincent Stimper, Marios Zacharias, Shuo Dong, Maciej Dendzik, Samuel Beaulieu, Bernhard Schölkopf, Martin Wolf, Laurenz Rettig, Christian Carbogno, et al. A machine learning route between band mapping and band structure. arXiv preprint arXiv:2005.10210, 2020.

[61] K. Medjanik, O. Fedchenko, S. Chernov, D. Kutnyakhov, M. Ellguth, A. Oelsner, B. Schönhense, T. R. F. Peixoto, P. Lutz, C.-H. Min, F. Reinert, S. Däster, Y. Acremann, J. Viefhaus, W. Wurth, H. J. Elmers, and G. Schönhense. Direct 3D mapping of the Fermi surface and Fermi velocity. Nature Materials, 16(6):615-621, June 2017.

[62] G. Schönhense, K. Medjanik, S. Chernov, D. Kutnyakhov, O. Fedchenko, M. Ellguth, D. Vasilyev, A. Zaporozhchenko-Zymaková, D. Panzer, A. Oelsner, et al. Spin-filtered time-of-flight k-space microscopy of ir-towards the "complete" photoemission experiment. Ultramicroscopy, 183:19-29, 2017.

[63] G. Schönhense, S. Babenkov, D. Vasilyev, H.-J. Elmers, and K. Medjanik. Single-hemisphere photoelectron momentum microscope with time-of-flight recording. Review of Scientific Instruments, 91(12):123110, 2020.

[64] S. Passlack, S. Mathias, O. Andreyev, D. Mittnacht, M. Aeschlimann, and M. Bauer. Space charge effects in photoemission with a low repetition, high intensity femtosecond laser source. Journal of Applied Physics, 100(2):024912, July 2006.

[65] S. Hellmann, K. Rossnagel, M. Marczynski-Bühlow, and L. Kipp. Vacuum space-charge effects in solid-state photoemission. Phys. Rev. B, 79:035402, Jan 2009.

[66] L.-P. Oloff, K. Hanff, A. Stange, G. Rohde, F. Diekmann, M. Bauer, and K. Rossnagel. Pump laser-induced space-charge effects in hhg-driven timeand angle-resolved photoelectron spectroscopy. Journal of Applied Physics, 119(22):225106, 2016.

[67] B. Schönhense, K. Medjanik, O. Fedchenko, S. Chernov, M. Ellguth, D. Vasilyev, A. Oelsner, J. Viefhaus, D. Kutnyakhov, W. Wurth, H. J. Elmers, and G. Schönhense. Multidimensional photoemission spectroscopy - the spacecharge limit. New Journal of Physics, 20(3):033004, mar 2018. 
[68] Zenghu Chang, Andy Rundquist, Haiwen Wang, Margaret M. Murnane, and Henry C. Kapteyn. Generation of Coherent Soft X Rays at $2.7 \mathrm{~nm}$ Using High Harmonics. Physical Review Letters, 79(16):2967-2970, 1997.

[69] M. Hentschel, R. Kienberger, Ch. Spielmann, G. A. Reider, N. Milosevic, T. Brabec, P. Corkum, U. Heinzmann, M. Drescher, and F. Krausz. Attosecond metrology. Nature, 414(6863):509-513, 2001.

[70] Ch. Spielmann, N. H. Burnett, S. Sartania, R. Koppitsch, M. Schnörer, C. Kan, M. Lenzner, P. Wobrauschek, and F. Krausz. Generation of Coherent X-rays in the Water Window Using 5-Femtosecond Laser Pulses. Science, 278(5338):661-664, 1997.

[71] Ferenc Krausz and Misha Ivanov. Attosecond physics. Rev. Mod. Phys., 81(1):163, February 2009.

[72] Tenio Popmintchev, Ming-Chang Chen, Dimitar Popmintchev, Paul Arpin, Susannah Brown, Skirmantas Alisauskas, Giedrius Andriukaitis, Tadas Balciunas, Oliver D. Mücke, Audrius Pugzlys, Andrius Baltuska, Bonggu Shim, Samuel E. Schrauth, Alexander Gaeta, Carlos Hernandez-Garcia, Luis Plaja, Andreas Becker, Agnieszka Jaron-Becker, Margaret M. Murnane, and Henry C. Kapteyn. Bright coherent ultrahigh harmonics in the keV x-ray regime from mid-infrared femtosecond lasers. Science (New York, N.Y.), 336(6086):1287-91, 2012.

[73] M. Harmand, R. Coffee, M. R. Bionta, M. Chollet, D. French, D. Zhu, D. M. Fritz, H. T. Lemke, N. Medvedev, B. Ziaja, S. Toleikis, and M. Cammarata. Achieving few-femtosecond time-sorting at hard X-ray free-electron lasers. $\mathrm{Na}$ ture Photonics, 7(3):215-218, March 2013.

[74] Joseph Duris, Siqi Li, Taran Driver, Elio G. Champenois, James P. MacArthur, Alberto A. Lutman, Zhen Zhang, Philipp Rosenberger, Jeff W. Aldrich, Ryan Coffee, Giacomo Coslovich, Franz-Josef Decker, James M. Glownia, Gregor Hartmann, Wolfram Helml, Andrei Kamalov, Jonas Knurr, Jacek Krzywinski, Ming-Fu Lin, Jon P. Marangos, Megan Nantel, Adi Natan, Jordan T. O’Neal, Niranjan Shivaram, Peter Walter, Anna Li Wang, James J. Welch, Thomas J. A. Wolf, Joseph Z. Xu, Matthias F. Kling, Philip H. Bucksbaum, Alexander Zholents, Zhirong Huang, James P. Cryan, and Agostino Marinelli. Tunable isolated attosecond $\mathrm{X}$-ray pulses with gigawatt peak power from a free-electron laser. Nature Photonics, 14(1):30-36, January 2020.

[75] Steffen Hädrich, Arno Klenke, Jan Rothhardt, Manuel Krebs, Armin Hoffmann, Oleg Pronin, Vladimir Pervak, Jens Limpert, and Andreas Tünnermann. High photon flux table-top coherent extreme-ultraviolet source. Nature Photonics, 8(10):779-783, 2014. 
[76] Steffen Hädrich, Jan Rothhardt, Manuel Krebs, Stefan Demmler, Arno Klenke, Andreas Tünnermann, and Jens Limpert. Single-pass high harmonic generation at high repetition rate and photon flux. Journal of Physics B: Atomic, Molecular and Optical Physics, 49(17):172002, 2016.

[77] G. S. Matthijs Jansen, Marius Keunecke, Marten Düvel, Christina Möller, David Schmitt, Wiebke Bennecke, Jasmin Kappert, Daniel Steil, D. Russell Luke, Sabine Steil, and Stefan Mathias. Efficient orbital imaging based on ultrafast momentum microscopy and sparsity-driven phase retrieval. New Journal of Physics, 2020.

[78] Marius Keunecke, Christina Möller, David Schmitt, Hendrik Nolte, G. S. Matthijs Jansen, Marcel Reutzel, Marie Gutberlet, Gyula Halasi, Daniel Steil, Sabine Steil, and Stefan Mathias. Time-resolved momentum microscopy with a $1 \mathrm{mhz}$ high-harmonic extreme ultraviolet beamline. Review of Scientific Instruments, 91(6):063905, 2020.

[79] Marius Keunecke, Marcel Reutzel, David Schmitt, Alexander Osterkorn, Tridev A. Mishra, Christina Möller, Wiebke Bennecke, G. S. Matthijs Jansen, Daniel Steil, Salvatore R. Manmana, Sabine Steil, Stefan Kehrein, and Stefan Mathias. Electromagnetic dressing of the electron energy spectrum of au(111) at high momenta. Phys. Rev. B, 102:161403, Oct 2020.

[80] Marius Keunecke, David Schmitt, Marcel Reutzel, Marius Weber, Christina Möller, G. S. Matthijs Jansen, Tridev A. Mishra, Alexander Osterkorn, Wiebke Bennecke, Klaus Pierz, Hans Werner Schumacher, Davood Momeni Pakdehi, Daniel Steil, Salvatore R. Manmana, Sabine Steil, Stefan Kehrein, Hans Christian Schneider, and Stefan Mathias. Direct access to auger recombination in graphene. arXiv preprint arXiv:2012.01256, 2020.

[81] Julien Madéo, Michael K. L. Man, Chakradhar Sahoo, Marshall Campbell, Vivek Pareek, E. Laine Wong, Abdullah Al-Mahboob, Nicholas S. Chan, Arka Karmakar, Bala Murali Krishna Mariserla, Xiaoqin Li, Tony F. Heinz, Ting Cao, and Keshav M. Dani. Directly visualizing the momentum-forbidden dark excitons and their dynamics in atomically thin semiconductors. Science, 370(6521):1199-1204, 2020.

[82] D. Kutnyakhov, R. P. Xian, M. Dendzik, M. Heber, F. Pressacco, S. Y. Agustsson, L. Wenthaus, H. Meyer, S. Gieschen, G. Mercurio, A. Benz, K. Bühlman, S. Däster, R. Gort, D. Curcio, K. Volckaert, M. Bianchi, Ch. Sanders, J. A. Miwa, S. Ulstrup, A. Oelsner, C. Tusche, Y.-J. Chen, D. Vasilyev, K. Medjanik, G. Brenner, S. Dziarzhytski, H. Redlin, B. Manschwetus, S. Dong, J. Hauer, L. Rettig, F. Diekmann, K. Rossnagel, J. Demsar, H.-J. Elmers, Ph. Hofmann, R. Ernstorfer, G. Schönhense, Y. Acremann, and W. Wurth. 
Time- and momentum-resolved photoemission studies using time-of-flight momentum microscopy at a free-electron laser. Review of Scientific Instruments, 91(1):013109, January 2020.

[83] Maciej Dendzik, R. Patrick Xian, Enrico Perfetto, Davide Sangalli, Dmytro Kutnyakhov, Shuo Dong, Samuel Beaulieu, Tommaso Pincelli, Federico Pressacco, Davide Curcio, Steinn Ymir Agustsson, Michael Heber, Jasper Hauer, Wilfried Wurth, Günter Brenner, Yves Acremann, Philip Hofmann, Martin Wolf, Andrea Marini, Gianluca Stefanucci, Laurenz Rettig, and Ralph Ernstorfer. Observation of an excitonic mott transition through ultrafast corecum-conduction photoemission spectroscopy. Phys. Rev. Lett., 125:096401, Aug 2020.

[84] Florian Haag, Tobias Eul, Philip Thielen, Norman Haag, Benjamin Stadtmüller, and Martin Aeschlimann. Time-resolved two-photon momentum microscopy - a new approach to study hot carrier lifetimes in momentum space. Review of Scientific Instruments, 90(10):103104, 2019.

[85] J. Maklar, S. Dong, S. Beaulieu, T. Pincelli, M. Dendzik, Y. W. Windsor, R. P. Xian, M. Wolf, R. Ernstorfer, and L. Rettig. A quantitative comparison of time-of-flight momentum microscopes and hemispherical analyzers for time- and angle-resolved photoemission spectroscopy experiments. Review of Scientific Instruments, 91(12):123112, 2020.

[86] Samuel Beaulieu, Shuo Dong, Nicolas Tancogne-Dejean, Maciej Dendzik, Tommaso Pincelli, Julian Maklar, R. Patrick Xian, Michael A. Sentef, Martin Wolf, Angel Rubio, Laurenz Rettig, and Ralph Ernstorfer. Ultrafast light-induced lifshitz transition. arXiv preprint arXiv:2003.04059, 2020.

[87] Robert Wallauer, Miriam Raths, Klaus Stallberg, Lasse Münster, Dominik Brandstetter, Xiaosheng Yang, Jens Güdde, Peter Puschnig, Serguei Soubatch, Christian Kumpf, Francois C. Bocquet, Frank Stefan Tautz, and Ulrich Höfer. Tracing orbital images on ultrafast time scales. arXiv preprint arXiv:2010.02599, 2020.

[88] Takashi Oka and Sota Kitamura. Floquet engineering of quantum materials. Annual Review of Condensed Matter Physics, 10:387-408, 2019.

[89] Mark S. Rudner and Netanel H. Lindner. Band structure engineering and non-equilibrium dynamics in floquet topological insulators. Nature Reviews Physics, 2(5):229-244, 2020.

[90] Jon H. Shirley. Interaction of a quantum system with a strong oscillating field. $\mathrm{PhD}$ thesis, California Institute of Technology, 1963.

[91] Jon H. Shirley. Solution of the schrödinger equation with a hamiltonian periodic in time. Phys. Rev., 138:B979-B987, May 1965. 
[92] Fahad Mahmood, Ching-Kit Chan, Zhanybek Alpichshev, Dillon Gardner, Young Lee, Patrick A. Lee, and Nuh Gedik. Selective scattering between Floquet-Bloch and Volkov states in a topological insulator. Nat Phys, 12(4):306-310, 2016.

[93] Sang Tae Park. Interference in Floquet-Volkov transitions. Physical Review A, 90(1):013420, 2014.

[94] Luis Miaja-Avila, C. Lei, M. Aeschlimann, J. L. Gland, M. M. Murnane, H. C. Kapteyn, and G. Saathoff. Laser-assisted photoelectric effect from surfaces. Physical review letters, 97(11):113604, 2006.

[95] G. Saathoff, L. Miaja-Avila, M. Aeschlimann, M. M. Murnane, and H. C. Kapteyn. Laser-assisted photoemission from surfaces. Physical Review A, 77(2):022903, 2008.

[96] A. Einstein. Über einen die erzeugung und verwandlung des lichtes betreffenden heuristischen gesichtspunkt. Annalen der Physik, 322(6):132-148, 1905 .

[97] H. Hertz. Über einen einfluss des ultravioletten lichtes auf die electrische entladung. Annalen der Physik, 267(8):983-1000, 1887.

[98] R. Yen, J. M. Liu, N. Bloembergen, T. K. Yee, J. G. Fujimoto, and M. M. Salour. Picosecond laser interaction with metallic zirconium. Applied Physics Letters, 40(2):185-187, 1982.

[99] R. T. Williams, T. R. Royt, J. C. Rife, J. P. Long, and M. N. Kabler. Picosecond time-resolved photoelectron spectroscopy of ZnTe. Journal of Vacuum Science and Technology, 21(2):509-513, 1982.

[100] R. Haight and J. A. Silberman. Surface intervalley scattering on GaAs(110): Direct observation with picosecond laser photoemission. Phys. Rev. Lett., 62:815-818, Feb 1989.

[101] R. Haight, J. Bokor, J. Stark, R. H. Storz, R. R. Freeman, and P. H. Bucksbaum. Picosecond time-resolved photoemission study of the $\operatorname{InP}(110)$ surface. Phys. Rev. Lett., 54:1302-1305, Mar 1985.

[102] G. D. Mahan. Excitons in metals: Infinite hole mass. Phys. Rev., 163:612-617, Nov 1967.

[103] G. D. Mahan. Theory of photoemission in simple metals. Phys. Rev. B, 2:4334-4350, Dec 1970.

[104] Peter J. Feibelman and D. E. Eastman. Photoemission spectroscopycorrespondence between quantum theory and experimental phenomenology. Phys. Rev. B, 10:4932-4947, Dec 1974. 
[105] J. B. Pendry. Theory of photoemission. Surface science, 57(2):679-705, 1976.

[106] J. F. L. Hopkinson, J. B. Pendry, and D. J. Titterington. Calculation of photoemission spectra for surfaces of solids. Computer Physics Communications, 19(1):69-92, 1980.

[107] M. A. Hoyland and R. G. Jordan. An experimental and theoretical study of the photoemission from Ni (110). Journal of Physics: Condensed Matter, 3(10):1337, 1991.

[108] T. Miller, W. E. McMahon, and T.-C. Chiang. Interference between bulk and surface photoemission transitions in Ag (111). Physical review letters, 77(6):1167, 1996.

[109] Lars Hedin, John Michiels, and John Inglesfield. Transition from the adiabatic to the sudden limit in core-electron photoemission. Phys. Rev. B, 58:1556515582, Dec 1998.

[110] F. Boschini, D. Bugini, M. Zonno, M. Michiardi, R. P. Day, E. Razzoli, B. Zwartsenberg, M. Schneider, E. H. da Silva Neto, S. Dal Conte, et al. Role of matrix elements in the time-resolved photoemission signal. New Journal of Physics, 22(2):023031, 2020.

[111] Carl Neil Berglund and William Edward Spicer. Photoemission studies of copper and silver: theory. Physical Review, 136(4A):A1030, 1964.

[112] E. Wigner and J. Bardeen. Theory of the work functions of monovalent metals. Phys. Rev., 48:84-87, Jul 1935.

[113] John Bardeen. Theory of the work function. II. the surface double layer. Phys. Rev., 49:653-663, May 1936.

[114] Janusz Chrzanowski and Marcin Matuszak. The double dipole layer and work function of metals. Applied Surface Science, 527:146767, 2020.

[115] N. D. Lang and W Kohn. Theory of metal surfaces: work function. Physical Review B, 3(4):1215, 1971.

[116] E. O. Kane. Implications of crystal momentum conservation in photoelectric emission for band-structure measurements. Physical Review Letters, 12(4):97, 1964 .

[117] Eberhard Dietz and D. E. Eastman. Symmetry method for the absolute determination of energy-band dispersions e $(\mathrm{k})$ using angle-resolved photoelectron spectroscopy. Physical Review Letters, 41(24):1674, 1978.

[118] N. E. Christensen. Angle resolved photoemission: Appearance angles. Solid State Communications, 38(4):309-312, 1981. 
[119] R. Courths. Absolute determination of electronic band structure of copper by angle-resolved photoemission. Solid State Communications, 40(5):529-533, 1981.

[120] André Eckardt and Egidijus Anisimovas. High-frequency approximation for periodically driven quantum systems from a floquet-space perspective. New journal of physics, 17(9):093039, 2015.

[121] Hideo Sambe. Steady states and quasienergies of a quantum-mechanical system in an oscillating field. Phys. Rev. A, 7:2203-2213, Jun 1973.

[122] Umberto De Giovannini and Hannes Hübener. Floquet analysis of excitations in materials. Journal of Physics: Materials, 3(1):012001, 2019.

[123] Aaron Farrell, A. Arsenault, and T. Pereg-Barnea. Dirac cones, floquet side bands, and theory of time-resolved angle-resolved photoemission. Physical Review B, 94(15):155304, 2016.

[124] Marin Bukov, Luca D'Alessio, and Anatoli Polkovnikov. Universal highfrequency behavior of periodically driven systems: from dynamical stabilization to floquet engineering. Advances in Physics, 64(2):139-226, 2015.

[125] M. A. Sentef, M. Claassen, A. F. Kemper, B. Moritz, T. Oka, J. K. Freericks, and T. P. Devereaux. Theory of floquet band formation and local pseudospin textures in pump-probe photoemission of graphene. Nature communications, $6(1): 1-8,2015$.

[126] Felix Bloch. Über die quantenmechanik der elektronen in kristallgittern. Zeitschrift für physik, 52(7-8):555-600, 1929.

[127] L. Miaja-Avila, J. Yin, S. Backus, Guido Saathoff, M. Aeschlimann, M. M. Murnane, and H. C. Kapteyn. Ultrafast studies of electronic processes at surfaces using the laser-assisted photoelectric effect with long-wavelength dressing light. Physical Review A, 79(3):030901, 2009.

[128] D. M. Wolkow. Über eine klasse von lösungen der diracschen gleichung. Zeitschrift für Physik, 94(3-4):250-260, 1935.

[129] Lars Bojer Madsen. Strong-field approximation in laser-assisted dynamics. American journal of physics, 73(1):57-62, 2005.

[130] Jan Conrad Baggesen and Lars Bojer Madsen. Theory for time-resolved measurements of laser-induced electron emission from metal surfaces. Physical Review A, 78(3):032903, 2008.

[131] Changmin Lee, Timm Rohwer, Edbert J. Sie, Alfred Zong, Edoardo Baldini, Joshua Straquadine, Philip Walmsley, Dillon Gardner, Young S. Lee, 
Ian R. Fisher, et al. High resolution time-and angle-resolved photoemission spectroscopy with 11 ev laser pulses. Review of Scientific Instruments, 91(4):043102, 2020.

[132] Markus Drescher, Michael Hentschel, R Kienberger, Matthias Uiberacker, Vladislav Yakovlev, Armin Scrinzi, Th. Westerwalbesloh, U. Kleineberg, Ulrich Heinzmann, and Ferenc Krausz. Time-resolved atomic inner-shell spectroscopy. Nature, 419(6909):803-807, 2002.

[133] P. M. Paul, E. S. Toma, P. Breger, G. Mullot, F. Augé, Ph. Balcou, H. G. Muller, and P. Agostini. Observation of a train of attosecond pulses from high harmonic generation. Science, 292(5522):1689-1692, 2001.

[134] Andrius Baltuška, Th. Udem, M. Uiberacker, M. Hentschel, Eleftherios Goulielmakis, Ch. Gohle, Ronald Holzwarth, V. S. Yakovlev, A. Scrinzi, Th. W. Hänsch, et al. Attosecond control of electronic processes by intense light fields. Nature, 421(6923):611-615, 2003.

[135] L. Miaja-Avila, Guido Saathoff, S. Mathias, J. Yin, M. Bauer, M. Aeschlimann, M. M. Murnane, H. C. Kapteyn, et al. Direct measurement of core-level relaxation dynamics on a surface-adsorbate system. Physical Review Letters, 101(4):046101, 2008.

[136] Stefan Neppl, Ralph Ernstorfer, Adrian L. Cavalieri, C. Lemell, G. Wachter, Elisabeth Magerl, E. M. Bothschafter, Michael Jobst, Michael Hofstetter, Ulf Kleineberg, et al. Direct observation of electron propagation and dielectric screening on the atomic length scale. Nature, 517(7534):342-346, 2015.

[137] Zhensheng Tao, Cong Chen, Tibor Szilvási, Mark Keller, Manos Mavrikakis, Henry Kapteyn, and Margaret Murnane. Direct time-domain observation of attosecond final-state lifetimes in photoemission from solids. Science, 353(6294):62-67, 2016.

[138] C. A. Arrell, J. Ojeda, L. Mewes, J. Grilj, F. Frassetto, L. Poletto, F. Van Mourik, and M. Chergui. Laser-assisted photoelectric effect from liquids. Physical review letters, 117(14):143001, 2016.

[139] Carsten Winterfeldt, Christian Spielmann, and Gustav Gerber. Colloquium: Optimal control of high-harmonic generation. Rev. Mod. Phys., 80:117-140, Jan 2008.

[140] Tenio Popmintchev, Ming-Chang Chen, Paul Arpin, Margaret M. Murnane, and Henry C. Kapteyn. The attosecond nonlinear optics of bright coherent x-ray generation. Nature Photonics, 4(12):822-832, 2010. 
[141] A. McPherson, G. Gibson, H. Jara, U. Johann, T. S. Luk, I. A. McIntyre, K. Boyer, and C. K. Rhodes. Studies of multiphoton production of vacuumultraviolet radiation in the rare gases. J. Opt. Soc. Am. B, 4(4):595-601, Apr 1987.

[142] M. Ferray, Anne L'Huillier, X. F. Li, L. A. Lompre, G. Mainfray, and C. Manus. Multiple-harmonic conversion of $1064 \mathrm{~nm}$ radiation in rare gases. Journal of Physics B: Atomic, Molecular and Optical Physics, 21(3):L31, 1988.

[143] P. B. Corkum. Plasma perspective on strong field multiphoton ionization. Phys. Rev. Lett., 71:1994-1997, Sep 1993.

[144] K. J. Schafer K. C. Kulander and J. L. Krause. Super-Intense Laser-Atom Physics. Springer US, 1993.

[145] Maciej Lewenstein, Ph. Balcou, M. Yu. Ivanov, Anne L'huillier, and Paul B. Corkum. Theory of high-harmonic generation by low-frequency laser fields. Physical Review A, 49(3):2117, 1994.

[146] L. V. Keldysh et al. Ionization in the field of a strong electromagnetic wave. Sov. Phys. JETP, 20(5):1307-1314, 1965.

[147] Vasileios-Marios Gkortsas, Siddharth Bhardwaj, Chien-Jen Lai, Kyung-Han Hong, Edilson L. Falcão-Filho, and Franz X. Kärtner. Interplay of mulitphoton and tunneling ionization in short-wavelength-driven high-order harmonic generation. Physical Review A, 84(1):013427, 2011.

[148] Chien-Jen Lai, Giovanni Cirmi, Kyung-Han Hong, Jeffrey Moses, ShuWei Huang, Eduardo Granados, Phillip Keathley, Siddharth Bhardwaj, and Franz X. Kärtner. Wavelength scaling of high harmonic generation close to the multiphoton ionization regime. Physical Review Letters, 111(7):073901, 2013 .

[149] H. R. Reiss. Unsuitability of the keldysh parameter for laser fields. Physical Review A, 82(2):023418, 2010.

[150] E. Balogh. Macroscopic study and control of high-order harmonic and attosecond pulse generation in noble gases. PhD thesis, University of Szeged, 2014 .

[151] Tenio Popmintchev. Tunable ultrafast coherent light in the soft and hard $X$ ray regions of the spectrum: Phase matching of extreme high-order harmonic generation. PhD thesis, University of Colorado, 2009.

[152] Christoph Heyl. Scaling and gating attosecond pulse generation. PhD thesis, Lund University, 2014. 
[153] Marie Gutberlet. High-harmonic generation in the tight focusing regime. Master's thesis, Georg-August-Universität Göttingen, 2020.

[154] Germaine Arend. Femtosecond ultraviolet light sources for time-resolved photoelectron spectroscopy. Master's thesis, Georg-August-Universität Göttingen, 2020 .

[155] K. S. Novoselov, A. K. Geim, S. V. Morozov, D. Jiang, Y. Zhang, S. V. Dubonos, I. V. Grigorieva, and A. A. Firsov. Electric field effect in atomically thin carbon films. Science, 306(5696):666-669, 2004.

[156] Dimitrios G. Papageorgiou, Ian A. Kinloch, and Robert J. Young. Mechanical properties of graphene and graphene-based nanocomposites. Progress in Materials Science, 90:75 - 127, 2017.

[157] Changgu Lee, Xiaoding Wei, Jeffrey W. Kysar, and James Hone. Measurement of the elastic properties and intrinsic strength of monolayer graphene. Science, 321(5887):385-388, 2008.

[158] K.I. Bolotin, K.J. Sikes, Z. Jiang, M. Klima, G. Fudenberg, J. Hone, P. Kim, and H.L. Stormer. Ultrahigh electron mobility in suspended graphene. Solid State Communications, 146(9):351 - 355, 2008.

[159] Alexander S. Mayorov, Roman V. Gorbachev, Sergey V. Morozov, Liam Britnell, Rashid Jalil, Leonid A. Ponomarenko, Peter Blake, Kostya S. Novoselov, Kenji Watanabe, Takashi Taniguchi, and A. K. Geim. Micrometer-scale ballistic transport in encapsulated graphene at room temperature. Nano Letters, 11(6):2396-2399, 2011. PMID: 21574627.

[160] Alexander A. Balandin, Suchismita Ghosh, Wenzhong Bao, Irene Calizo, Desalegne Teweldebrhan, Feng Miao, and Chun Ning Lau. Superior thermal conductivity of single-layer graphene. Nano Letters, 8(3):902-907, 2008. PMID: 18284217.

[161] Alexander A Balandin. Thermal properties of graphene and nanostructured carbon materials. Nature materials, 10(8):569-581, 2011.

[162] A. H. Castro Neto, F. Guinea, N. M. R. Peres, K. S. Novoselov, and A. K. Geim. The electronic properties of graphene. Rev. Mod. Phys., 81:109-162, Jan 2009.

[163] T. Schumann, M. Dubslaff, M. H. Oliveira, M. Hanke, J. M. J. Lopes, and H. Riechert. Effect of buffer layer coupling on the lattice parameter of epitaxial graphene on sic(0001). Phys. Rev. B, 90:041403, Jul 2014.

[164] Monica Pozzo, Dario Alfè, Paolo Lacovig, Philip Hofmann, Silvano Lizzit, and Alessandro Baraldi. Thermal expansion of supported and freestanding 
graphene: Lattice constant versus interatomic distance. Phys. Rev. Lett., 106:135501, Mar 2011.

[165] Daniel R. Cooper, Benjamin D'Anjou, Nageswara Ghattamaneni, Benjamin Harack, Michael Hilke, Alexandre Horth, Norberto Majlis, Mathieu Massicotte, Leron Vandsburger, Eric Whiteway, et al. Experimental review of graphene. ISRN Condensed Matter Physics, 2012, 2012.

[166] P. R. Wallace. The band theory of graphite. Phys. Rev., 71:622-634, May 1947.

[167] Rupali Kundu. Tight-binding parameters for graphene. Modern Physics Letters B, 25(03):163-173, 2011.

[168] Jeil Jung and Allan H. MacDonald. Tight-binding model for graphene $\pi$-bands from maximally localized wannier functions. Phys. Rev. B, 87:195450, May 2013.

[169] Stephanie Reich, Janina Maultzsch, Christian Thomsen, and Pablo Ordejon. Tight-binding description of graphene. Physical Review B, 66(3):035412, 2002.

[170] R. S. Deacon, K.-C. Chuang, R. J. Nicholas, K. S. Novoselov, and A. K. Geim. Cyclotron resonance study of the electron and hole velocity in graphene monolayers. Phys. Rev. B, 76:081406, Aug 2007.

[171] Kostya S. Novoselov, S. V. Geim, Andre K .and Morozov, Da Jiang, Michail I. Katsnelson, I. V. Grigorieva, S. V. Dubonos, and A. A. Firsov. Twodimensional gas of massless dirac fermions in graphene. nature, 438(7065):197200, 2005 .

[172] W. S. Jung, C. S. Leem, Chul Kim, S. R. Park, S. Y. Park, B. J. Kim, E. Rotenberg, and C. Kim. Imaging the electron density in solids by using multi-brillouin-zone angle resolved photoelectron spectroscopy. Phys. Rev. B, 82:235105, Dec 2010.

[173] D. P. DiVincenzo and E. J. Mele. Self-consistent effective-mass theory for intralayer screening in graphite intercalation compounds. Physical Review B, 29(4):1685, 1984.

[174] M. I. Katsnelson, K. S. Novoselov, and A. K. Geim. Chiral tunnelling and the klein paradox in graphene. Nature physics, 2(9):620-625, 2006.

[175] S. Aeschlimann. Ultrafast Quasiparticle Dynamics in Graphene and 2D Heterostructures. Thesis, Universität Hamburg, 2018.

[176] Gordon W. Semenoff. Condensed-matter simulation of a three-dimensional anomaly. Physical Review Letters, 53(26):2449, 1984. 
[177] Cristina Bena and Gilles Montambaux. Remarks on the tight-binding model of graphene. New Journal of Physics, 11(9):095003, 2009.

[178] Anastasia Varlet, Ming-Hao Liu, Dominik Bischoff, Pauline Simonet, Takashi Taniguchi, Kenji Watanabe, Klaus Richter, Thomas Ihn, and Klaus Ensslin. Band gap and broken chirality in single-layer and bilayer graphene. physica status solidi (RRL)-Rapid Research Letters, 10(1):46-57, 2016.

[179] S. Aeschlimann, R. Krause, M. Chávez-Cervantes, H. Bromberger, R. Jago, E. Malić, A. Al-Temimy, C. Coletti, A. Cavalleri, and I. Gierz. Ultrafast momentum imaging of pseudospin-flip excitations in graphene. Phys. Rev. B, 96:020301, Jul 2017.

[180] Isabella Gierz, Jürgen Henk, Hartmut Höchst, Christian R. Ast, and Klaus Kern. Illuminating the dark corridor in graphene: Polarization dependence of angle-resolved photoemission spectroscopy on graphene. Physical Review B, 83(12):121408, 2011.

[181] Choongyu Hwang, Cheol-Hwan Park, David A. Siegel, Alexei V. Fedorov, Steven G. Louie, and Alessandra Lanzara. Direct measurement of quantum phases in graphene via photoemission spectroscopy. Phys. Rev. B, 84:125422, Sep 2011.

[182] Eric L. Shirley, L. J. Terminello, A. Santoni, and F. J. Himpsel. Brillouinzone-selection effects in graphite photoelectron angular distributions. Phys. Rev. B, 51:13614-13622, May 1995.

[183] Pourya Ayria, Ahmad R. T. Nugraha, Eddwi H. Hasdeo, Thomas R. Czank, Shin-ichiro Tanaka, and Riichiro Saito. Photon energy dependence of angle-resolved photoemission spectroscopy in graphene. Physical Review B, 92(19):195148, 2015.

[184] M. Trushin and J. Schliemann. Anisotropic photoconductivity in graphene. EPL (Europhysics Letters), 96(3):37006, oct 2011.

[185] Ermin Malic, Torben Winzer, Evgeny Bobkin, and Andreas Knorr. Microscopic theory of absorption and ultrafast many-particle kinetics in graphene. Phys. Rev. B, 84:205406, Nov 2011.

[186] A. Grüneis, R. Saito, Ge. G. Samsonidze, T. Kimura, M. A. Pimenta, A. Jorio, A. G. Souza Filho, G. Dresselhaus, and M. S. Dresselhaus. Inhomogeneous optical absorption around the k point in graphite and carbon nanotubes. Phys. Rev. B, 67:165402, Apr 2003.

[187] Xiao-Qing Yan, Jun Yao, Zhi-Bo Liu, Xin Zhao, Xu-Dong Chen, Chengmin Gao, Wei Xin, Yongsheng Chen, and Jian-Guo Tian. Evolution of anisotropicto-isotropic photoexcited carrier distribution in graphene. Phys. Rev. B, 90:134308, Oct 2014 . 
[188] M. Trushin, A. Grupp, G. Soavi, A. Budweg, D. De Fazio, U. Sassi, A. Lombardo, A. C. Ferrari, W. Belzig, A. Leitenstorfer, and D. Brida. Ultrafast pseudospin dynamics in graphene. Phys. Rev. B, 92:165429, Oct 2015.

[189] J. C. König-Otto, M. Mittendorff, T. Winzer, F. Kadi, E. Malic, A. Knorr, C. Berger, W. A. de Heer, A. Pashkin, H. Schneider, M. Helm, and S. Winnerl. Slow noncollinear coulomb scattering in the vicinity of the dirac point in graphene. Phys. Rev. Lett., 117:087401, Aug 2016.

[190] Minjung Kim, Ho Ang Yoon, Seungwoo Woo, Duhee Yoon, Sang Wook Lee, and Hyeonsik Cheong. Polarization dependence of photocurrent in a metalgraphene-metal device. Applied Physics Letters, 101(7):073103, 2012.

[191] Martin Mittendorff, Torben Winzer, Ermin Malic, Andreas Knorr, Claire Berger, Walter A. de Heer, Harald Schneider, Manfred Helm, and Stephan Winnerl. Anisotropy of excitation and relaxation of photogenerated charge carriers in graphene. Nano Letters, 14(3):1504-1507, 2014. PMID: 24559191.

[192] Ermin Malic, Torben Winzer, Florian Wendler, and Andreas Knorr. Review on carrier multiplication in graphene. physica status solidi (b), 253(12):23032310, 2016.

[193] Torben Winzer, Andreas Knorr, and Ermin Malic. Carrier multiplication in graphene. Nano letters, 10(12):4839-4843, 2010.

[194] Ermin Malic, Torben Winzer, Florian Wendler, Samuel Brem, Roland Jago, Andreas Knorr, Martin Mittendorff, Jacob C. König-Otto, Tobias Plötzing, Daniel Neumaier, et al. Carrier dynamics in graphene: Ultrafast many-particle phenomena. Annalen der Physik, 529(11):1700038, 2017.

[195] Isabella Gierz, Matteo Mitrano, Jesse C. Petersen, Cephise Cacho, I. C. Edmond Turcu, Emma Springate, Alexander Stöhr, Axel Köhler, Ulrich Starke, and Andrea Cavalleri. Population inversion in monolayer and bilayer graphene. Journal of Physics: Condensed Matter, 27(16):164204, 2015.

[196] Daniele Brida, Andrea Tomadin, Cristian Manzoni, Yong Jin Kim, Antonio Lombardo, Silvia Milana, Rahul Raveendran Nair, Kostya S. Novoselov, Andrea C. Ferrari, Giulio Cerullo, et al. Ultrafast collinear scattering and carrier multiplication in graphene. Nature communications, 4(1):1-9, 2013.

[197] Jens Christian Johannsen, Sören Ulstrup, Alberto Crepaldi, Federico Cilento, Michele Zacchigna, Jill A. Miwa, Cephise Cacho, Richard T. Chapman, Emma Springate, Felix Fromm, Christian Raidel, Thomas Seyller, Phil D. C. King, Fulvio Parmigiani, Marco Grioni, and Philip Hofmann. Tunable carrier multiplication and cooling in graphene. Nano letters, 15(1):326-31, 2014. 
[198] J. C. Johannsen, S. Ulstrup, F. Cilento, A. Crepaldi, M. Zacchigna, C. Cacho, I. C. E. Turcu, E. Springate, F. Fromm, C. Raidel, T. Seyller, F. Parmigiani, M. Grioni, and P. Hofmann. Direct view of hot carrier dynamics in graphene. Phys. Rev. Lett., 111(2):027403, 2013.

[199] Søren Ulstrup, Jens Christian Johannsen, Alberto Crepaldi, Federico Cilento, Michele Zacchigna, Cephise Cacho, Richard T. Chapman, Emma Springate, Felix Fromm, Christian Raidel, et al. Ultrafast electron dynamics in epitaxial graphene investigated with time-and angle-resolved photoemission spectroscopy. Journal of Physics: Condensed Matter, 27(16):164206, 2015.

[200] Isabella Gierz, Francesca Calegari, Sven Aeschlimann, M. Chávez Cervantes, C. Cacho, R. T. Chapman, E. Springate, S. Link, U. Starke, C. R. Ast, et al. Tracking primary thermalization events in graphene with photoemission at extreme time scales. Physical review letters, 115(8):086803, 2015.

[201] Jared H. Strait, Haining Wang, Shriram Shivaraman, Virgil Shields, Michael Spencer, and Farhan Rana. Very slow cooling dynamics of photoexcited carriers in graphene observed by optical-pump terahertz-probe spectroscopy. Nano letters, 11(11):4902-4906, 2011.

[202] M. Breusing, S. Kuehn, T. Winzer, E. Malić, F. Milde, N. Severin, J. P. Rabe, C. Ropers, A. Knorr, and T. Elsaesser. Ultrafast nonequilibrium carrier dynamics in a single graphene layer. Physical Review B, 83(15):153410, 2011.

[203] Petr A. Obraztsov, Maxim G. Rybin, Anastasia V. Tyurnina, Sergey V. Garnov, Elena D. Obraztsova, Alexander N. Obraztsov, and Yuri P. Svirko. Broadband light-induced absorbance change in multilayer graphene. Nano letters, 11(4):1540-1545, 2011.

[204] Ermin Malic, Torben Winzer, and Andreas Knorr. Efficient orientational carrier relaxation in optically excited graphene. Applied Physics Letters, 101(21):213110, 2012.

[205] S. Winnerl, M. Orlita, P. Plochocka, P. Kossacki, M. Potemski, T. Winzer, E. Malic, A. Knorr, M. Sprinkle, C. Berger, et al. Carrier relaxation in epitaxial graphene photoexcited near the dirac point. Physical review letters, 107(23):237401, 2011.

[206] Fabio Caruso, Dino Novko, and Claudia Draxl. Photoemission signatures of nonequilibrium carrier dynamics from first principles. Physical Review B, 101(3):035128, 2020.

[207] Justin C. W. Song, Michael Y. Reizer, and Leonid S. Levitov. Disorderassisted electron-phonon scattering and cooling pathways in graphene. Phys. Rev. Lett., 109:106602, Sep 2012. 
[208] Wang-Kong Tse and S. Das Sarma. Energy relaxation of hot dirac fermions in graphene. Phys. Rev. B, 79:235406, Jun 2009.

[209] Kostyantyn M. Borysenko, Jeffrey T. Mullen, E. A. Barry, S. Paul, Yuriy G. Semenov, J. M. Zavada, M. Buongiorno Nardelli, and Ki Wook Kim. Firstprinciples analysis of electron-phonon interactions in graphene. Physical Review $B, 81(12): 121412,2010$.

[210] Stephan Winnerl, F. Göttfert, Martin Mittendorff, H. Schneider, Manfred Helm, Torben Winzer, Ermin Malic, Andreas Knorr, Milan Orlita, Marek Potemski, et al. Time-resolved spectroscopy on epitaxial graphene in the infrared spectral range: relaxation dynamics and saturation behavior. Journal of Physics: Condensed Matter, 25(5):054202, 2013.

[211] Nicola Bonini, Michele Lazzeri, Nicola Marzari, and Francesco Mauri. Phonon anharmonicities in graphite and graphene. Physical review letters, 99(17):176802, 2007.

[212] Kristen Kaasbjerg, Kristian S. Thygesen, and Karsten W. Jacobsen. Unraveling the acoustic electron-phonon interaction in graphene. Physical Review B, 85(16):165440, 2012.

[213] Jahan M. Dawlaty, Shriram Shivaraman, Mvs Chandrashekhar, Farhan Rana, and Michael G. Spencer. Measurement of ultrafast carrier dynamics in epitaxial graphene. Applied Physics Letters, 92(4):042116, 2008.

[214] Thonimar V. Alencar, Mychel G. Silva, Leandro M. Malard, and Ana M. de Paula. Defect-induced supercollision cooling of photoexcited carriers in graphene. Nano letters, 14(10):5621-5624, 2014.

[215] Thomas Danz, Andreas Neff, John H. Gaida, Reiner Bormann, Claus Ropers, and Sascha Schäfer. Ultrafast sublattice pseudospin relaxation in graphene probed by polarization-resolved photoluminescence. Phys. Rev. B, 95:241412, Jun 2017.

[216] Torben Winzer, Andreas Knorr, Martin Mittendorff, Stephan Winnerl, MiaoBin Lien, Dong Sun, Theodore B. Norris, Manfred Helm, and Ermin Malic. Absorption saturation in optically excited graphene. Applied Physics Letters, 101(22):221115, 2012.

[217] Stephan Winnerl, Martin Mittendorff, Jacob C. König-Otto, Harald Schneider, Manfred Helm, Torben Winzer, Andreas Knorr, and Ermin Malic. Ultrafast processes in graphene: from fundamental manybody interactions to device applications. Annalen der Physik, 529(11):1700022, 2017. 
[218] Alexander Grüneis, Claudio Attaccalite, Ludger Wirtz, H. Shiozawa, R. Saito, Thomas Pichler, and Angel Rubio. Tight-binding description of the quasiparticle dispersion of graphite and few-layer graphene. Physical Review B, 78(20):205425, 2008.

[219] Pierluigi Cudazzo, Ilya V. Tokatly, and Angel Rubio. Dielectric screening in two-dimensional insulators: Implications for excitonic and impurity states in graphane. Physical Review B, 84(8):085406, 2011.

[220] D. M. Basko. Effect of anisotropic band curvature on carrier multiplication in graphene. Physical Review B, 87(16):165437, 2013.

[221] Andrea Tomadin, Daniele Brida, Giulio Cerullo, Andrea C. Ferrari, and Marco Polini. Nonequilibrium dynamics of photoexcited electrons in graphene: Collinear scattering, auger processes, and the impact of screening. Phys. Rev. $B$, 88:035430, Jul 2013.

[222] Georgy Alymov, Vladimir Vyurkov, Victor Ryzhii, Akira Satou, and Dmitry Svintsov. Auger recombination in dirac materials: A tangle of many-body effects. Physical Review B, 97(20):205411, 2018.

[223] Justin C. W. Song, Klaas J. Tielrooij, Frank H. L. Koppens, and Leonid S. Levitov. Photoexcited carrier dynamics and impact-excitation cascade in graphene. Phys. Rev. B, 87:155429, Apr 2013.

[224] Dmitry Svintsov, Victor Ryzhii, Akira Satou, Taiichi Otsuji, and Vladimir Vyurkov. Carrier-carrier scattering and negative dynamic conductivity in pumped graphene. Optics express, 22(17):19873-19886, 2014.

[225] Tsuneya Ando. Screening effect and impurity scattering in monolayer graphene. Journal of the Physical Society of Japan, 75(7):074716-074716, 2006 .

[226] E. H. Hwang and S. Das Sarma. Dielectric function, screening, and plasmons in two-dimensional graphene. Phys. Rev. B, 75:205418, May 2007.

[227] Valeri N Kotov, Bruno Uchoa, Vitor M Pereira, F Guinea, and AH Castro Neto. Electron-electron interactions in graphene: Current status and perspectives. Reviews of Modern Physics, 84(3):1067, 2012.

[228] Michael Schütt, P. M. Ostrovsky, I. V. Gornyi, and A. D. Mirlin. Coulomb interaction in graphene: Relaxation rates and transport. Physical Review B, 83(15):155441, 2011.

[229] Farhan Rana. Electron-hole generation and recombination rates for coulomb scattering in graphene. Phys. Rev. B, 76:155431, Oct 2007. 
[230] Roland Jago, Torben Winzer, and Ermin Malic. Recombination channels in optically excited graphene. physica status solidi (b), 252(11):2456-2460, 2015.

[231] Anuj Girdhar and Jean-Pierre Leburton. Soft carrier multiplication by hot electrons in graphene. Applied physics letters, 99(4):043107, 2011.

[232] Shuntaro Tani, François Blanchard, and Koichiro Tanaka. Ultrafast carrier dynamics in graphene under a high electric field. Physical review letters, 109(16):166603, 2012.

[233] Torben Winzer and Ermin Malić. Impact of auger processes on carrier dynamics in graphene. Physical Review B, 85(24):241404, 2012.

[234] T. Winzer and E. Malic. The impact of pump fluence on carrier relaxation dynamics in optically excited graphene. Journal of Physics: Condensed Matter, 25(5):054201, 2013.

[235] Faris Kadi, Torben Winzer, Andreas Knorr, and Ermin Malic. Impact of doping on the carrier dynamics in graphene. Scientific reports, 5:16841, 2015.

[236] Torben Winzer, Roland Jago, and Ermin Malic. Experimentally accessible signatures of auger scattering in graphene. Physical Review B, 94(23):235430, 2016.

[237] M. Mohr, J. Maultzsch, E. Dobardžić, S. Reich, I. Milošević, M. Damnjanović, A. Bosak, M. Krisch, and C. Thomsen. Phonon dispersion of graphite by inelastic x-ray scattering. Phys. Rev. B, 76:035439, Jul 2007.

[238] S. Butscher, F. Milde, M. Hirtschulz, E. Malić, and A. Knorr. Hot electron relaxation and phonon dynamics in graphene. Applied Physics Letters, 91(20):203103, 2007.

[239] Meng Xing Na, Arthur K. Mills, Fabio Boschini, Matteo Michiardi, Benjamin Nosarzewski, Ryan P. Day, Elia Razzoli, Alexander Sheyerman, Michael Schneider, Giorgio Levy, et al. Direct determination of mode-projected electron-phonon coupling in the time domain. Science, 366(6470):1231-1236, 2019.

[240] Ludger Wirtz and Angel Rubio. The phonon dispersion of graphite revisited. Solid State Communications, 131(3-4):141-152, 2004.

[241] U. Monteverde, J. Pal, M.A. Migliorato, M. Missous, U. Bangert, R. Zan, R. Kashtiban, and D. Powell. Under pressure: Control of strain, phonons and bandgap opening in rippled graphene. Carbon, 91:266 - 274, 2015.

[242] M. Nisoli, S. De Silvestri, O. Svelto, R. Szipöcs, K. Ferencz, Ch. Spielmann, S. Sartania, and F. Krausz. Compression of high-energy laser pulses below 5 fs. Opt. Lett., OL, 22(8):522-524, April 1997. 
[243] Jan Rothhardt, Steffen Hädrich, Arno Klenke, Stefan Demmler, Armin Hoffmann, Thomas Gotschall, Tino Eidam, Manuel Krebs, Jens Limpert, and Andreas Tünnermann. 53W average power few-cycle fiber laser system generating soft x rays up to the water window. Opt. Lett., OL, 39(17):5224-5227, September 2014.

[244] David Schmitt. Photoemission momentum microscopy with a high-repetitive hhg light source. Master's thesis, Georg-August-Universität Göttingen, 2018.

[245] Amelie Schulte. High-repetitive xuv pulse generation with high harmonic generation. Bachelor's thesis, Georg-August-Universität Göttingen, 2017.

[246] Rick Trebino, Kenneth W. DeLong, David N. Fittinghoff, John N. Sweetser, Marco A. Krumbügel, Bruce A. Richman, and Daniel J. Kane. Measuring ultrashort laser pulses in the time-frequency domain using frequency-resolved optical gating. Review of Scientific Instruments, 68(9):3277-3295, 1997.

[247] Rick Trebino. Frequency-resolved optical gating: the measurement of ultrashort laser pulses. Springer Science \& Business Media, 2012.

[248] Patrick O'shea, Mark Kimmel, Xun Gu, and Rick Trebino. Highly simplified device for ultrashort-pulse measurement. Optics letters, 26(12):932-934, 2001.

[249] Cheng Tien Chiang, Alexander Blättermann, Michael Huth, Jürgen Kirschner, and Wolf Widdra. High-order harmonic generation at $4 \mathrm{MHz}$ as a light source for time-of-flight photoemission spectroscopy. Applied Physics Letters, 101(7), 2012 .

[250] M. Neviere, D. Maystre, and W. R. Hunter. On the use of classical and conical diffraction mountings for xuv gratings. J. Opt. Soc. Am., 68(8):1106-1113, Aug 1978.

[251] Oleg Pronin, Vladimir Pervak, Ernst Fill, Jens Rauschenberger, Ferenc Krausz, and Alexander Apolonski. Ultrabroadband efficient intracavity xuv output coupler. Opt. Express, 19(11):10232-10240, May 2011.

[252] Jeromy Hollenshead and Leonard Klebanoff. Modeling radiation-induced carbon contamination of extreme ultraviolet optics. Journal of Vacuum Science \& Technology B, 24(64), 2006.

[253] Tsuneharu Koide, Tetsuo Shidara, Mihiro Yanagihara, and Shigero Sato. Resuscitation of carbon-contaminated mirrors and gratings by oxygen-discharge cleaning . 2 : Efficiency recovery in the 100-1000-eV range. Applied optics, 27(20):4305-4313, 1988.

[254] Roger W. C. Hansen, Mark Bissen, Dan Wallace, Jeff Wolske, and Tom Miller. Ultraviolet / ozone cleaning of carbon-contaminated optics. Applied optics, 32(22):4114-4116, 1993. 
[255] S. Babenkov, K. Medjanik, D. Vasilyev, S. Chernov, C. Schlueter, A. Gloskovskii, Yu Matveyev, W. Drube, B. Schönhense, K. Rossnagel, et al. High-accuracy bulk electronic bandmapping with eliminated diffraction effects using hard x-ray photoelectron momentum microscopy. Communications Physics, 2(1):1-8, 2019 .

[256] Christian Tusche, Ying-Jiun Chen, Lukasz Plucinski, and Claus M Schneider. From photoemission microscopy to an "all-in-one" photoemission experiment. e-Journal of Surface Science and Nanotechnology, 18:48-56, 2020.

[257] Masato Kotsugi, W. Kuch, F. Offi, L. I. Chelaru, and J. Kirschner. Microspectroscopic two-dimensional fermi surface mapping using a photoelectron emission microscope. Review of scientific instruments, 74(5):2754-2758, 2003.

[258] G. Schönhense. Imaging of magnetic structures by photoemission electron microscopy. Journal of Physics: Condensed Matter, 11(48):9517, 1999.

[259] Marten Christopher Patt. Bulk and surface sensitive energy-filtered photoemission microscopy using synchrotron radiation for the study of resistive switching memories. PhD thesis, Jülich, 2016. Dissertation, Universität Duisburg-Essen, 2016.

[260] Claus M. Schneider and Gerd Schönhense. Investigating surface magnetism by means of photoexcitation electron emission microscopy. Reports on Progress in Physics, 65(12):1785, 2002.

[261] Ray F. Egerton et al. Physical principles of electron microscopy, volume 56. Springer, 2005.

[262] A. Oelsner, O. Schmidt, M. Schicketanz, M. Klais, G. Schönhense, V. Mergel, O. Jagutzki, and H. Schmidt-Böcking. Microspectroscopy and imaging using a delay line detector in time-of-flight photoemission microscopy. Review of Scientific Instruments, 72(10):3968-3974, 2001.

[263] M. I. Green, P. F. Kenealy, and G. B. Beard. Fast-timing measurements using a chevron microchannel plate electron multiplier. Nuclear Instruments and Methods, 126(2):175-179, 1975.

[264] Knut Müller-Caspary, Andreas Oelsner, and Pavel Potapov. Stem strain measurement from a stream of diffraction patterns recorded on a pixel-free delayline detector. Microscopy and Microanalysis, 22(S3):520-521, 2016.

[265] Constantin Witte. Build up and comissioning of a sample preparation chamber for experiments in time resolved pes. Bachelor's thesis, Georg-AugustUniversität Göttingen, 2017. 
[266] Hendrik Nolte. Visualization and analysis of multidimensional photoelectron spectroscopy data. Master's thesis, Georg-August-Universität Göttingen, 2019.

[267] Rui Patrick Xian, Yves Acremann, Steinn Ymir Agustsson, Maciej Dendzik, Kevin Bühlmann, Davide Curcio, Dmytro Kutnyakhov, Frederico Pressacco, Michael Heber, Shuo Dong, Jure Demsar, Wilfried Wurth, Philip Hofmann, Martin Wolf, Laurenz Rettig, and Ralph Ernstorfer. An open-source, distributed workflow for band mapping data in multidimensional photoemission spectroscopy. arXiv preprint arXiv:1909.07714, September 2019.

[268] V. Stimper, S. Bauer, R. Ernstorfer, B. Schölkopf, and R. P. Xian. Multidimensional contrast limited adaptive histogram equalization. IEEE Access, 7:165437-165447, 2019.

[269] R. Patrick Xian, Laurenz Rettig, and Ralph Ernstorfer. Symmetry-guided nonrigid registration: The case for distortion correction in multidimensional photoemission spectroscopy. Ultramicroscopy, 202:133-139, 2019.

[270] Wheeler P. Davey. Precision measurements of the lattice constants of twelve common metals. Phys. Rev., 25:753-761, Jun 1925.

[271] G. Schönhense, K. Medjanik, C. Tusche, M. de Loos, B. van der Geer, M. Scholz, F. Hieke, N. Gerken, J. Kirschner, and W. Wurth. Correction of the deterministic part of space-charge interaction in momentum microscopy of charged particles. Ultramicroscopy, 159:488-496, 2015.

[272] Mattias Kruskopf, Davood Momeni Pakdehi, Klaus Pierz, Stefan Wundrack, Rainer Stosch, Thorsten Dziomba, Martin Götz, Jens Baringhaus, Johannes Aprojanz, Christoph Tegenkamp, et al. Comeback of epitaxial graphene for electronics: large-area growth of bilayer-free graphene on sic. 2D Materials, 3(4):041002, 2016.

[273] D. Momeni Pakdehi, J. Aprojanz, A. Sinterhauf, K. Pierz, M. Kruskopf, P. Willke, J. Baringhaus, J. P. Stöckmann, G. A. Traeger, F. Hohls, C. Tegenkamp, M. Wenderoth, F. J. Ahlers, and H. W. Schumacher. Minimum resistance anisotropy of epitaxial graphene on sic. ACS Applied Materials \&6 Interfaces, 10(6):6039-6045, 2018.

[274] Aaron Bostwick, Taisuke Ohta, Thomas Seyller, Karsten Horn, and Eli Rotenberg. Quasiparticle dynamics in graphene. Nature physics, 3(1):36-40, 2007.

[275] D. Niesner, Th Fauster, J. I. Dadap, N. Zaki, K. R. Knox, P. C. Yeh, R. Bhandari, R. M. Osgood, M. Petrović, and M. Kralj. Trapping surface electrons on graphene layers and islands. Physical Review B, 85(8):081402, 2012. 
[276] N. Armbrust, J. Güdde, P. Jakob, and Ulrich Höfer. Time-resolved two-photon photoemission of unoccupied electronic states of periodically rippled graphene on ru(0001). Phys. Rev. Lett., 108(5):056801, 2012.

[277] Shijing Tan, Adam Argondizzo, Cong Wang, Xuefeng Cui, and Hrvoje Petek. Ultrafast multiphoton thermionic photoemission from graphite. Phys. Rev. X, 7(1):011004, 2017.

[278] G. Rohde, A. Stange, A. Müller, M. Behrendt, L. P. Oloff, K. Hanff, T. J Albert, P. Hein, K. Rossnagel, and M. Bauer. Ultrafast formation of a fermidirac distributed electron gas. Physical Review Letters, 121(25):256401, 2018.

[279] Takashi Oka and Sota Kitamura. Floquet Engineering of Quantum Materials. Annual Review of Condensed Matter Physics, 10(1):387-408, 2019.

[280] Gerd Schönhense, Katerina Medjanik, and Hans-Joachim Elmers. Space-, time- and spin-resolved photoemission. Journal of Electron Spectroscopy and Related Phenomena, 200:94-118, April 2015.

[281] Florian Haag, Tobias Eul, Philip Thielen, Norman Haag, Benjamin Stadtmüller, and Martin Aeschlimann. Time-resolved two-photon momentum microscopy - A new approach to study hot carrier lifetimes in momentum space. Review of Scientific Instruments, 90(10):103104, October 2019.

[282] C. Tusche, P. Goslawski, D. Kutnyakhov, M. Ellguth, K. Medjanik, H. J. Elmers, S. Chernov, R. Wallauer, D. Engel, A. Jankowiak, et al. Multi-mhz time-of-flight electronic bandstructure imaging of graphene on $\operatorname{Ir}(111)$. Applied physics letters, 108(26):261602, 2016.

[283] O. Fedchenko, A. Winkelmann, K. Medjanik, S. Babenkov, D. Vasilyev, S. Chernov, C. Schlueter, A. Gloskovskii, Yu Matveyev, W. Drube, B. Schönhense, H. J. Elmers, and G. Schönhense. High-resolution hard-x-ray photoelectron diffraction in a momentum microscope - the model case of graphite. New J. Phys., 21(11):113031, November 2019.

[284] Marcel Reutzel, Andi Li, and Hrvoje Petek. Coherent two-dimensional multiphoton photoelectron spectroscopy of metal surfaces. Physical Review X, 9(1):011044, 2019.

[285] M. Merschdorf, W. Pfeiffer, A. Thon, S. Voll, and G. Gerber. Photoemission from multiply excited surface plasmons in Ag nanoparticles. Appl. Phys. A, 71(5):547-552, 2000.

[286] Marcel Reutzel, Andi Li, Branko Gumhalter, and Hrvoje Petek. Nonlinear plasmonic photoelectron response of ag(111). Physical Review Letters, 123(1):017404, 2019. 
[287] Zhipei Sun, Tawfique Hasan, Felice Torrisi, Daniel Popa, Giulia Privitera, Fengqiu Wang, Francesco Bonaccorso, Denis M. Basko, and Andrea C. Ferrari. Graphene mode-locked ultrafast laser. ACS Nano, 4(2):803-810, 2010. PMID: 20099874.

[288] T. Hertel, E. Knoesel, A. Hotzel, M. Wolf, and G. Ertl. Femtosecond timeresolved photoemission of electron dynamics in surface rydberg states. Journal of Vacuum Science $\& 3$ Technology A, 15(3):1503-1509, 1997.

[289] W. Berthold, U. Höfer, P. Feulner, E. V. Chulkov, V. M. Silkin, and P. M. Echenique. Momentum-resolved lifetimes of image-potential states on cu(100). Phys. Rev. Lett., 88(5):056805, 2002.

[290] J. A. Sobota, S. Yang, J. G. Analytis, Y. L. Chen, I. R. Fisher, P. S. Kirchmann, and Z. X. Shen. Ultrafast optical excitation of a persistent surfacestate population in the topological insulator $\mathrm{Bi}_{2} \mathrm{Se}_{3}$. Physical Review Letters, 108(11):117403, 2012.

[291] D. N. Basov, R. D. Averitt, and D. Hsieh. Towards properties on demand in quantum materials. Nature materials, 16(11):1077-1088, 2017.

[292] Yoshinori Tokura, Masashi Kawasaki, and Naoto Nagaosa. Emergent functions of quantum materials. Nature Physics, 13(11):1056-1068, 2017.

[293] Y. H. Wang, Hadar Steinberg, Pablo Jarillo-Herrero, and Nuh Gedik. Observation of floquet-bloch states on the surface of a topological insulator. Science, 342(6157):453-457, 2013.

[294] Ro-Ya Liu, Yu Ogawa, Peng Chen, Kenichi Ozawa, Takeshi Suzuki, Masaru Okada, Takashi Someya, Yukiaki Ishida, Kozo Okazaki, Shik Shin, et al. Femtosecond to picosecond transient effects in wse 2 observed by pump-probe angle-resolved photoemission spectroscopy. Scientific reports, 7(1):1-7, 2017.

[295] N.W. Ashcroft and D.N. Mermin. Festkörperphysik. Oldenbourg Wissenschaftsverlag, 2012.

[296] J. K. Freericks, H. R. Krishnamurthy, and Th. Pruschke. Theoretical description of time-resolved photoemission spectroscopy: Application to pump-probe experiments. Phys. Rev. Lett., 102:136401, Mar 2009.

[297] M. N. Faraggi, M. S. Gravielle, and D. M. Mitnik. Interaction of ultrashort laser pulses with metal surfaces: Impulsive jellium-volkov approximation versus the solution of the time-dependent schrödinger equation. Phys. Rev. A, 76(1):012903, 2017.

[298] T. Rangel, D. Kecik, P. E. Trevisanutto, G. M. Rignanese, H. Van Swygenhoven, and V. Olevano. Band structure of gold from many-body perturbation theory. Physical Review B, 86(12):125125, 2012. 
[299] F. Roth, T. Arion, H. Kaser, A. Gottwald, and W. Eberhardt. Angle resolved photoemission from ag and au single crystals: Final state lifetimes in the attosecond range. Journal of Electron Spectroscopy and Related Phenomena, 224:84-92, 2017.

[300] F. Reinert, G. Nicolay, S. Schmidt, D. Ehm, and S. Hüfner. Direct measurements of the l-gap surface states on the (111) face of noble metals by photoelectron spectroscopy. Phys. Rev. B, 63(11):115415, 2001.

[301] Robert L. Olmon, Brian Slovick, Timothy W. Johnson, David Shelton, SangHyun Oh, Glenn D. Boreman, and Markus B. Raschke. Optical dielectric function of gold. Phys. Rev. B, 86(23):235147, 2012.

[302] M. A. B. Whitaker. Use of the fresnel equations in the theory of angle-resolved photoemission. Journal of Physics C: Solid State Physics, 11(4):L151, 1978.

[303] Peter J. Feibelman. Comment on recent theories of photoemission. Surface Science, 46(2):558-566, 1974.

[304] Marcel Reutzel, Andi Li, Zehua Wang, and Hrvoje Petek. Coherent multidimensional photoelectron spectroscopy of ultrafast quasiparticle dressing by light. Nature Communications, 11(1):2230, 2020.

[305] C. M. Schneider and J. Kirschner. Spin-and angle-resolved photoelectron spectroscopy from solid surfaces with circularly polarized light. Critical Reviews in Solid State and Material Sciences, 20(3):179-283, 1995.

[306] R. H. Williams, G. P. Srivastava, and I. T. McGovern. Photoelectron spectroscopy of solids and their surfaces. Reports on Progress in Physics, 43(12):1357, 1980.

[307] Harald Wern and Ralf Courths. Photoemission from Ag (110) (PARUPS): Angle-of-incidence effect on intensities and use of fresnel equations. Surface Science, 162(1-3):29-33, 1985.

[308] Peter J. Feibelman. Self-consistent calculation of the surface photoelectric effect. Physical Review Letters, 34(17):1092, 1975.

[309] Peter J. Feibelman. Microscopic calculation of electromagnetic fields in refraction at a jellium-vacuum interface. Physical Review B, 12(4):1319, 1975.

[310] Peter J. Feibelman. Surface electromagnetic fields. Progress in Surface Science, 12(4):287-407, 1982.

[311] Harry J. Levinson, E. W. Plummer, and Peter J. Feibelman. Effects on photoemission of the spatially varying photon field at a metal surface. Physical Review Letters, 43(13):952, 1979. 
[312] M. Lucchini, L. Castiglioni, L. Kasmi, P. Kliuiev, A. Ludwig, M. Greif, J. Osterwalder, M. Hengsberger, L. Gallmann, and U. Keller. Light-matter interaction at surfaces in the spatiotemporal limit of macroscopic models. Phys. Rev. Lett., 115:137401, Sep 2015.

[313] A. Liebsch. Dynamical screening at simple-metal surfaces. Physical Review B, 36(14):7378, 1987.

[314] J. Reimann, Stefan Schlauderer, C. P. Schmid, Fabian Langer, Sebastian Baierl, K. A. Kokh, O. E. Tereshchenko, A. Kimura, Christoph Lange, Jens Güdde, et al. Subcycle observation of lightwave-driven dirac currents in a topological surface band. Nature, 562(7727):396-400, 2018.

[315] Ervand Kandelaki and Mark S. Rudner. Many-body dynamics and gap opening in interacting periodically driven systems. Physical Review Letters, 121(3):036801, 2018.

[316] S. A. Sato, U. De Giovannini, S. Aeschlimann, I. Gierz, H. Hübener, and A. Rubio. Floquet states in dissipative open quantum systems. Journal of Physics B: Atomic, Molecular and Optical Physics, 53(22):225601, 2020.

[317] Michael Schüler, Umberto De Giovannini, Hannes Hübener, Angel Rubio, Michael A. Sentef, Thomas P. Devereaux, and Philipp Werner. How circular dichroism in time- and angle-resolved photoemission can be used to spectroscopically detect transient topological states in graphene. Phys. Rev. X, 10:041013, Oct 2020 .

[318] Mona H. Kalthoff, Götz S. Uhrig, and J. K. Freericks. Emergence of floquet behavior for lattice fermions driven by light pulses. Physical Review B, 98(3):035138, 2018.

[319] Umberto De Giovannini, Hannes Hübener, and Angel Rubio. Monitoring electron-photon dressing in $\mathrm{WSe}_{2}$. Nano Letters, 16(12):7993-7998, 2016.

[320] Luca Pirro, Anuj Girdhar, Yusuf Leblebici, and Jean-Pierre Leburton. Impact ionization and carrier multiplication in graphene. Journal of Applied Physics, 112(9):093707, 2012.

[321] Jan Rothhardt, Steffen Hädrich, Stefan Demmler, Manuel Krebs, Stephan Fritzsche, Jens Limpert, and Andreas Tünnermann. Enhancing the macroscopic yield of narrow-band high-order harmonic generation by fano resonances. Physical review letters, 112(23):233002, 2014.

[322] Janina Felter, Jana Wolters, François C. Bocquet, F. Stefan Tautz, and Christian Kumpf. Momentum microscopy on the micrometer scale: photoemission micro-tomography applied to single molecular domains. Journal of Physics: Condensed Matter, 31(11):114003, January 2019. 
[323] Kin Fai Mak, Jie Shan, and Tony F. Heinz. Electronic structure of fewlayer graphene: Experimental demonstration of strong dependence on stacking sequence. Phys. Rev. Lett., 104:176404, Apr 2010.

[324] Fabian R. Geisenhof, Felix Winterer, Stefan Wakolbinger, Tobias D. Gokus, Yasin C. Durmaz, Daniela Priesack, Jakob Lenz, Fritz Keilmann, Kenji Watanabe, Takashi Taniguchi, Raúl Guerrero-Avilés, Marta Pelc, Andres Ayuela, and R. Thomas Weitz. Anisotropic strain-induced soliton movement changes stacking order and band structure of graphene multilayers: Implications for charge transport. ACS Applied Nano Materials, 2(9):6067-6075, 2019.

[325] R. Thomas Weitz, M. T. Allen, B. E. Feldman, J. Martin, and A. Yacoby. Broken-symmetry states in doubly gated suspended bilayer graphene. Science, 330(6005):812-816, 2010.

[326] Michael K. L. Man, Athanasios Margiolakis, Skylar Deckoff-Jones, Takaaki Harada, E. Laine Wong, M. Bala Murali Krishna, Julien Madéo, Andrew Winchester, Sidong Lei, Robert Vajtai, Pulickel M. Ajayan, and Keshav M. Dani. Imaging the motion of electrons across semiconductor heterojunctions. Nature Nanotechnology, 12(1):36-40, 2017.

[327] Philip Kahl, Simone Wall, Christian Witt, Christian Schneider, Daniela Bayer, Alexander Fischer, Pascal Melchior, Michael Horn-von Hoegen, Martin Aeschlimann, and Frank Meyer zu Heringdorf. Normal-incidence photoemission electron microscopy (NI - PEEM) for imaging surface plasmon polaritons. Plasmonics, 9(6):1401-1407, 2014.

[328] Philip Kahl, Daniel Podbiel, Christian Schneider, Andreas Makris, Simon Sindermann, Christian Witt, Deirdre Kilbane, Michael Horn-von Hoegen, Martin Aeschlimann, and Frank Meyer zu Heringdorf. Direct observation of surface plasmon polariton propagation and interference by time-resolved imaging in normal-incidence two photon photoemission microscopy. Plasmonics, 13(1):239-246, 2018.

[329] Yanan Dai, Zhikang Zhou, Atreyie Ghosh, Roger SK Mong, Atsushi Kubo, Chen-Bin Huang, and Hrvoje Petek. The birth of a plasmonic topological quasiparticle on the nanofemto scale. arXiv preprint arXiv:1912.03831, 2019.

[330] Kai Siegbahn, Ulrik Gelius, Hans Siegbahn, and Erik Olson. Angular distribution of electrons in ESCA spectra from a single crystal. Physica Scripta, 1(5-6):272, 1970.

[331] C. S. Fadley and S. Å. L. Bergström. Angular distribution of photoelectrons from a metal single crystal. Physics Letters A, 35(5):375-376, 1971. 
[332] C. S. Fadley, M. A. Van Hove, Z. Hussain, and A. P. Kaduwela. Photoelectron diffraction: new dimensions in space, time, and spin. Journal of electron spectroscopy and related phenomena, 75:273-297, 1995.

[333] D. P. Woodruff and A. M. Bradshaw. Adsorbate structure determination on surfaces using photoelectron diffraction. Reports on Progress in Physics, 57(10):1029, 1994.

[334] Ofer Kfir, Eliyahu Bordo, Gil Ilan Haham, Oren Lahav, Avner Fleischer, and Oren Cohen. In-line production of a bi-circular field for generation of helically polarized high-order harmonics. Applied Physics Letters, 108(21):211106, 2016.

[335] Tingting Fan, Patrik Grychtol, Ronny Knut, Carlos Hernández-García, Daniel D. Hickstein, Dmitriy Zusin, Christian Gentry, Franklin J. Dollar, Christopher A. Mancuso, Craig W. Hogle, et al. Bright circularly polarized soft x-ray high harmonics for x-ray magnetic circular dichroism. Proceedings of the National Academy of Sciences, 112(46):14206-14211, 2015.

[336] Ofer Kfir, Sergey Zayko, Christina Nolte, Murat Sivis, Marcel Möller, Birgit Hebler, Sri Sai Phani Kanth Arekapudi, Daniel Steil, Sascha Schäfer, Manfred Albrecht, et al. Nanoscale magnetic imaging using circularly polarized highharmonic radiation. Science advances, 3(12):eaao4641, 2017.

[337] W. Kuch, L. I. Chelaru, F. Offi, Masato Kotsugi, and J. Kirschner. Magnetic dichroisms in absorption and photoemission for magnetic characterization in x-ray photoelectron emission microscopy. Journal of Vacuum Science 63 Technology B: Microelectronics and Nanometer Structures Processing, Measurement, and Phenomena, 20(6):2543-2549, 2002.

[338] J. Miguel, J. Sánchez-Barriga, D. Bayer, J. Kurde, B. Heitkamp, M. Piantek, F. Kronast, M. Aeschlimann, H. A. Dürr, and W. Kuch. Time-resolved magnetization dynamics of cross-tie domain walls in permalloy microstructures. Journal of Physics: Condensed Matter, 21(49):496001, 2009.

[339] Olivier Boulle, Jan Vogel, Hongxin Yang, Stefania Pizzini, Dayane de Souza Chaves, Andrea Locatelli, Tevfik Onur Menteş, Alessandro Sala, Liliana D Buda-Prejbeanu, Olivier Klein, et al. Room-temperature chiral magnetic skyrmions in ultrathin magnetic nanostructures. Nature nanotechnology, 11(5):449, 2016.

[340] Kumari Gaurav Rana, Rafael Lopes Seeger, Sandra Ruiz-Gómez, Roméo Juge, Qiang Zhang, Van Tuong Pham, Mohamed Belmeguenai, Stéphane Auffret, Michael Foerster, Lucia Aballe, et al. Room temperature skyrmions in an exchange biased antiferromagnet. arXiv preprint arXiv:2009.14796, 2020. 
[341] Yasser A. Shokr, Oliver Sandig, Mustafa Erkovan, Bin Zhang, Matthias Bernien, Ahmet A. Ünal, Florian Kronast, Umut Parlak, Jan Vogel, and Wolfgang Kuch. Steering of magnetic domain walls by single ultrashort laser pulses. Physical Review B, 99(21):214404, 2019.

[342] B. T. Thole, G. Van der Laan, and G. A. Sawatzky. Strong magnetic dichroism predicted in the $\mathrm{M} \mathrm{4,5} \mathrm{x}$-ray absorption spectra of magnetic rare-earth materials. Physical review letters, 55(19):2086, 1985.

[343] Petr Němec, Manfred Fiebig, Tobias Kampfrath, and Alexey V. Kimel. Antiferromagnetic opto-spintronics. Nature Physics, 14(3):229-241, 2018.

[344] Peter Wadley, Bryn Howells, J. Železnỳ, Carl Andrews, Victoria Hills, Richard P. Campion, Vit Novák, K. Olejník, F. Maccherozzi, S. S. Dhesi, et al. Electrical switching of an antiferromagnet. Science, 351(6273):587-590, 2016 .

[345] Marc F. Tesch, Dominik Legut, H.-Ch. Mertins, Markus C. Gilbert, Christine Jansing, Jaroslav Hamrle, Jan Rusz, Peter M. Oppeneer, Daniel E. Bürgler, Claus M. Schneider, et al. Magnetocrystalline anisotropy in x-ray magnetic linear dichroism at the $3 \mathrm{p}$ edges of crystalline fe thin films. Physical Review $B, 89(14): 140404,2014$.

[346] Sergio Valencia, Armin Kleibert, Andreas Gaupp, Jan Rusz, Dominik Legut, Joachim Bansmann, Wolfgang Gudat, and Peter M Oppeneer. Quadratic x-ray magneto-optical effect upon reflection in a near-normal-incidence configuration at the m edges of 3 d-transition metals. Physical review letters, 104(18):187401, 2010 .

[347] M. Plötzing, R. Adam, C. Weier, L. Plucinski, S. Eich, S. Emmerich, M. Rollinger, M. Aeschlimann, S. Mathias, and C. M. Schneider. Spin-resolved photoelectron spectroscopy using femtosecond extreme ultraviolet light pulses from high-order harmonic generation. Review of Scientific Instruments, 87(4), 2016.

[348] Steffen Eich, Moritz Plötzing, Markus Rollinger, Sebastian Emmerich, Roman Adam, Cong Chen, Henry Cornelius Kapteyn, Margaret M. Murnane, Lukasz Plucinski, Daniel Steil, Benjamin Stadtmüller, Mirko Cinchetti, Martin Aeschlimann, Claus M. Schneider, and Stefan Mathias. Band structure evolution during the ultrafast ferromagnetic-paramagnetic phase transition in cobalt. Science advances, 3(3):e1602094, 2017.

[349] R. Gort, K. Bühlmann, S. Däster, G. Salvatella, N. Hartmann, Y. Zemp, S. Holenstein, C. Stieger, A. Fognini, T. U. Michlmayr, T. Bähler, A. Vaterlaus, and Y. Acremann. Early stages of ultrafast spin dynamics in a $3 d$ ferromagnet. Phys. Rev. Lett., 121:087206, Aug 2018. 
[350] S. Ogawa, H. Nagano, H. Petek, and A. P. Heberle. Optical dephasing in Cu (111) measured by interferometric two-photon time-resolved photoemission. Physical review letters, 78(7):1339, 1997.

[351] S. Ogawa, H. Nagano, and H. Petek. Phase and energy relaxation in an antibonding surface state: Cs/Cu (111). Physical review letters, 82(9):1931, 1999.

[352] Hannes Offenbacher, Daniel Lüftner, Thomas Ules, Eva Maria Reinisch, Georg Koller, Peter Puschnig, and Michael G. Ramsey. Orbital tomography: Molecular band maps, momentum maps and the imaging of real space orbitals of adsorbed molecules. Journal of Electron Spectroscopy and Related Phenomena, 204:92 - 101, 2015. Organic Electronics.

[353] Peter Puschnig, Stephen Berkebile, Alexander J. Fleming, Georg Koller, Konstantin Emtsev, Thomas Seyller, John D. Riley, Claudia Ambrosch-Draxl, Falko P. Netzer, and Michael G. Ramsey. Reconstruction of molecular orbital densities from photoemission data. Science, 326(5953):702-706, 2009.

[354] Stephen Berkebile, Thomas Ules, Peter Puschnig, Lorenz Romaner, Georg Koller, Alexander J. Fleming, Konstantin Emtsev, Thomas Seyller, Claudia Ambrosch-Draxl, Falko P. Netzer, and Michael G. Ramsey. A momentum space view of the surface chemical bond. Phys. Chem. Chem. Phys., 13:36043611, 2011.

[355] Daniel Lüftner, Thomas Ules, Eva Maria Reinisch, Georg Koller, Serguei Soubatch, F. Stefan Tautz, Michael G. Ramsey, and Peter Puschnig. Imaging the wave functions of adsorbed molecules. Proceedings of the National Academy of Sciences, 111(2):605-610, 2014.

[356] Frank Neese. The orca program system. WIREs Computational Molecular Science, 2(1):73-78, 2012.

[357] Isabella Gierz and Andrea Cavalleri. Electronic-structural dynamics in graphene. Structural Dynamics, 3(5):051301, 2016.

[358] Martin Claassen, Chunjing Jia, Brian Moritz, and Thomas P. Devereaux. Alloptical materials design of chiral edge modes in transition-metal dichalcogenides. Nature communications, 7(1):1-8, 2016.

[359] Tatsuhiko N. Ikeda and Masahiro Sato. General description for nonequilibrium steady states in periodically driven dissipative quantum systems. Science Advances, 6(27), 2020.

[360] Rémi Desbuquois, Michael Messer, Frederik Görg, Kilian Sandholzer, Gregor Jotzu, and Tilman Esslinger. Controlling the floquet state population and observing micromotion in a periodically driven two-body quantum system. Physical Review A, 96(5):053602, 2017. 
[361] Larry Bradley, Brigitta Sipőcz, Thomas Robitaille, Erik Tollerud, Zé Vinícius, Christoph Deil, Kyle Barbary, Tom J Wilson, Ivo Busko, Hans Moritz Günther, Mihai Cara, Simon Conseil, Azalee Bostroem, Michael Droettboom, E. M. Bray, Lars Andersen Bratholm, P. L. Lim, Geert Barentsen, Matt Craig, Sergio Pascual, Gabriel Perren, Johnny Greco, Axel Donath, Miguel de Val-Borro, Wolfgang Kerzendorf, Yoonsoo P. Bach, Benjamin Alan Weaver, Francesco D'Eugenio, Harrison Souchereau, and Leonardo Ferreira. astropy/photutils: 1.0.0, September 2020.

[362] Peter B. Stetson. DAOPHOT: A computer program for crowded-field stellar photometry. Publications of the Astronomical Society of the Pacific, 99(613):191, 1987.

[363] Aaron Bostwick, Taisuke Ohta, Jessica L McChesney, Konstantin V Emtsev, Thomas Seyller, Karsten Horn, and Eli Rotenberg. Symmetry breaking in few layer graphene films. New Journal of Physics, 9(10):385, 2007.

[364] C. M. Polley, Leif I. Johansson, H. Fedderwitz, T. Balasubramanian, M. Leandersson, J. Adell, Rositsa Yakimova, and Chariya Jacobi. Origin of the $\pi$-band replicas in the electronic structure of graphene grown on 4 h-sic (0001). Physical Review B, 99(11):115404, 2019.

[365] M. S. Nevius, M. Conrad, F. Wang, A. Celis, M. N. Nair, A. Taleb-Ibrahimi, A. Tejeda, and E. H. Conrad. Semiconducting graphene from highly ordered substrate interactions. Phys. Rev. Lett., 115:136802, Sep 2015.

[366] Kan Nakatsuji, Yuki Shibata, Ryota Niikura, Fumio Komori, Kouhei Morita, and Satoru Tanaka. Shape, width, and replicas of $\pi$ bands of single-layer graphene grown on si-terminated vicinal sic(0001). Phys. Rev. B, 82:045428, Jul 2010.

[367] Lunan Huang, Yun Wu, M. T. Hershberger, Daixiang Mou, Benjamin Schrunk, Michael C. Tringides, Myron Hupalo, and Adam Kaminski. Effects of moiré lattice structure on electronic properties of graphene. Phys. Rev. B, 96:035411, Jul 2017.

[368] Eli Rotenberg and Aaron Bostwick. Superlattice effects in graphene on $\mathrm{SiC}$ (0001) and Ir (111) probed by ARPES. Synthetic Metals, 210:85-94, 2015.

[369] I. Pletikosić, M. Kralj, P. Pervan, R. Brako, J. Coraux, A. T. N'Diaye, C. Busse, and T. Michely. Dirac cones and minigaps for graphene on $\operatorname{Ir}(111)$. Phys. Rev. Lett., 102:056808, Feb 2009.

[370] M. Bonitz, D. Kremp, D. C. Scott, R. Binder, W. D. Kraeft, and H. S. Köhler. Numerical analysis of non-Markovian effects in charge-carrier scattering: onetime versus two-time kinetic equations. J. Phys.: Cond. Mat., 8(33):6057, 1996. 
[371] K. Morawetz and H. S. Köhler. Formation of correlations and energyconservation at short times. Eur. Phys. J. A, 4:291, 1999.

[372] Hartmut Haug and Claudia Ell. Coulomb quantum kinetics in a dense electron gas. Phys. Rev. B, 46:2126, 1992.

[373] Ermin Malic and Andreas Knorr. Graphene and carbon nanotubes: ultrafast optics and relaxation dynamics, chapter 2, pages 9-50. John Wiley \& Sons, 2013. 


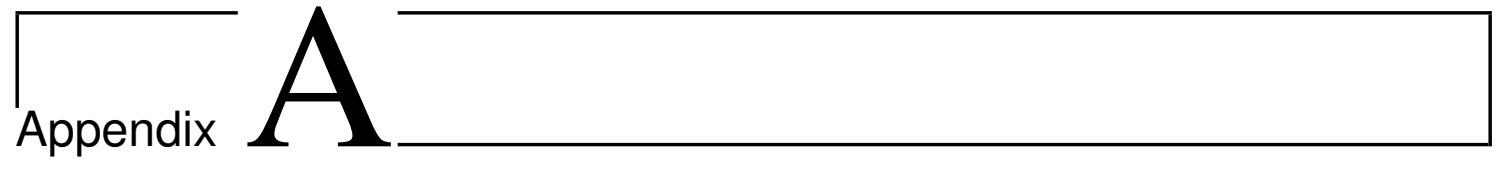

\section{Supplementary information for chapter 3}

\section{A.1. Spectrum and autocorrelation of the HHG driver}

A typical spectrum and an intensity autocorrelation measurement of the HHG driver at the generation point (i.e., after the lens and entrance window) is shown in figure A.1 confirming pulse duration of 65 fs (Gaussian).

(a)

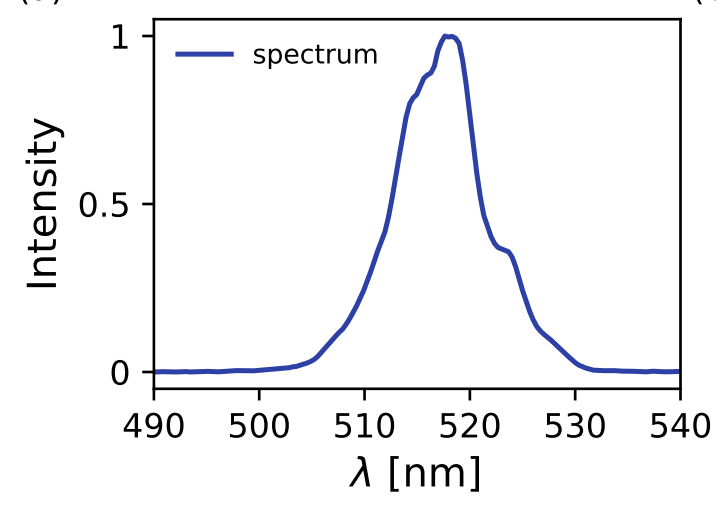

(b)

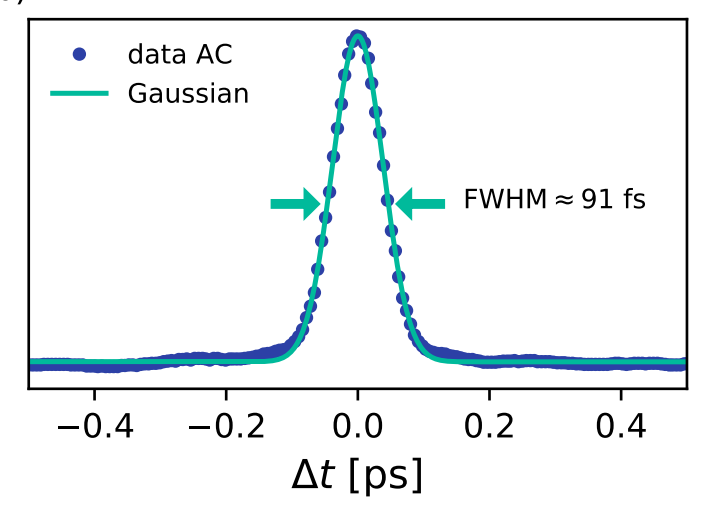

Figure A.1.: (a) Measured spectrum after frequency-doubling in a beta barium borate crystal ( $\beta$-BBO). (b) Autocorrelation measurement of the pulses (blue dots) and a Gaussian fit (light blue) which results in a FWHM $\approx 91 \mathrm{fs}$, which corresponds to pulse duration of $65 \mathrm{fs}$ (Gaussian).

\section{A.2. Metadata handling}

The additional metadata that is saved in each measurement run is summarized in figure A.2 showing the user sheet which is stored with each measurement run. Additionally, a startup and shutdown procedure are recommended. The main light 
source parameters are saved, i.e., the used photon energies $\hbar \omega$, polarizations, measured pulse durations $\tau$, delay stage positions (plus expected temporal overlap $t_{0}$ ), and used powers $P$.

From the momentum microscope the hexapod and aperture positions $(\mathrm{FA}=$ field aperture, $\mathrm{CA}=$ contrast aperture) are important parameters to reproduce a certain measurement geometry. The overall lens setting and in addition the most important lens voltages (i.e., extrator voltage $U_{\text {Ext. }}$, time-of-flight voltage $U_{\text {ToF }}$, sample voltage $U_{\text {Sample }}$ and resulting k-space magnifiation) are saved. Furthermore, the temperature $T$ at the back of the hexapod (related to the sample temperature) is kept.

The detector parameters, i.e., the used binnings in $\mathrm{x}, \mathrm{y}, \mathrm{t}\left(x_{b}, y_{b}, t_{b}\right)$, as well as, the time offset and -size ( $t_{b}$ offset and $t_{\text {size }}$, i.e., the area in binned time pixels which are saved in the .tif file) are stored likewise.

A general notes section, and one for each individual measurement allow to keep any additional important oberservations, comments, information with each measurement run.

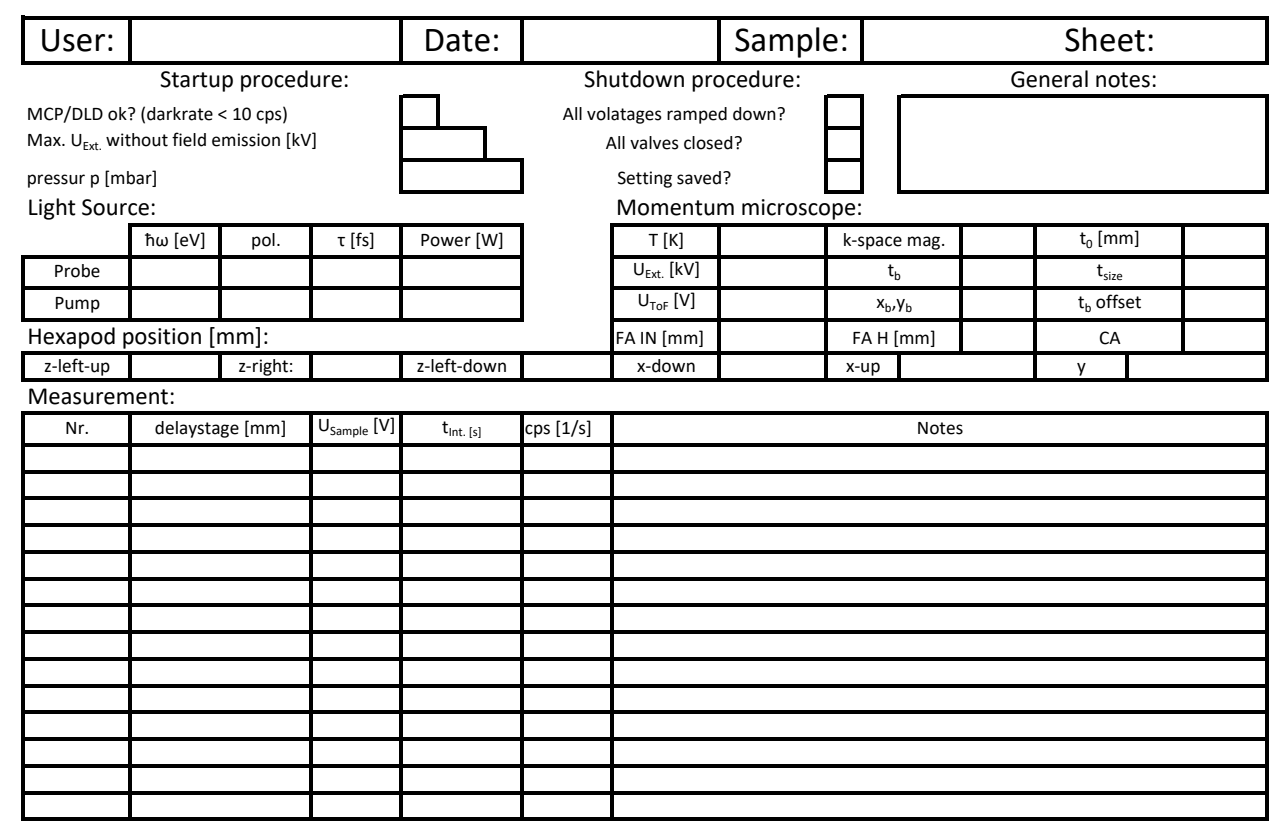

Figure A.2.: Metadata sheet that is stored with each measurement run. The details are clarified in the text. 
$T_{\text {Appendix }} \mathbf{B}$

\section{Supplementary information for chapter 4}

\section{B.1. Pump and probe spot sizes estimated by photoemission electron microscopy (PEEM)}

Pump and probe spot sizes were determined by using photoemission electron microscopy (PEEM). Therefore, again the chessy test specimen was used (compare sec. 4.2 to calibrate the lateral dimensions. The resulting images are shown in figure B.1 for (a) the pump beam $(1030 \mathrm{~nm})$, and (b) the EUV probe (13 ${ }^{\text {th }}$ harmonic $(26.5 \mathrm{eV}))$. The elliptical shape is most prominent for the EUV beam (b) due to the
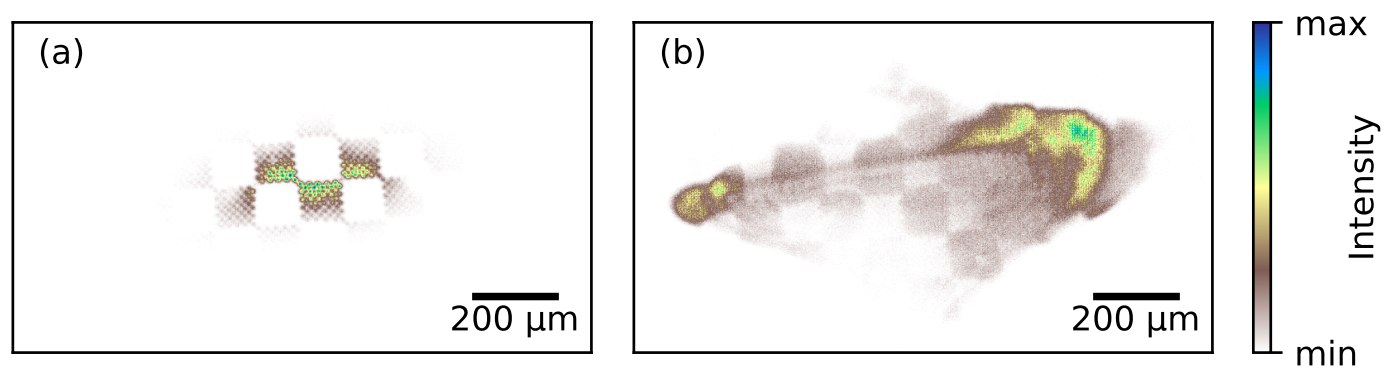

Figure B.1.: Real space images of the chessy test specimen showing the IR pump (a) and EUV probe (b) beams on the sample. The elliptical shaps are evident for both beams, but due to astigmatism in the EUV beam path it is more pronounced there. Moreover, the image of the EUV beam (b) is blurred due to the energetic width of the $13^{\text {th }}$ harmonic.

oblique angle of incidence and additional astigmatism in the EUV beampath. From these images the beamsizes have been estimated to be approximately $600 \times 900 \mu \mathrm{m}$ for the EUV probe and $100 \times 230 \mu \mathrm{m}$ for the pump beam. For the pump beam path this value is likely an underestimate since the signal arises due to multi-photon 
photoemission. Moreover, it is obvious that the image of the EUV beam (b) is not so clear compared to (a) due to the energetic bandwidth of the EUV beam.

\section{B.2. Optimization of spatial and temporal overlap in the momentum microscope}

The real space imaging allows for convenient optimization of the spatial overlap and placement of field apertures. This is shown exemplary in figure B.2 (b) where the driver of the HHG (515 nm, shown in the inset) is overlapped spatially with the pump beam (compare figure B.1 (a)). The field aperture (dashed black line) is placed so that only regions of good overlap are measured. This procedure can be used to get a fast estimate of the temporal overlap by scanning the delay stage while monitoring the multi-photon photoemission from the driver of the HHG plus the pump beam (figure B.2 (a)). The temporal width of $98 \pm 1$ fs (Gaussian) is related to the pulse lengths of both the pump beam and driver of the HHG and can be used as a fast check for the time resolution. Even though one has to note that this number is workfunction and therefore also sample dependent, due to the non-linearity in the photoemission process.
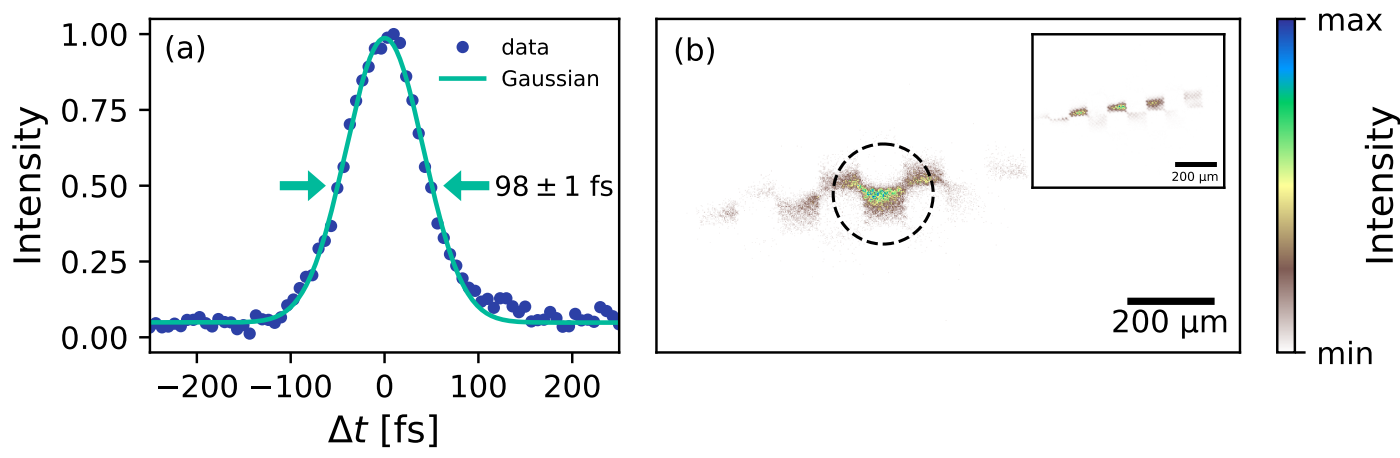

Figure B.2.: (a) Estimation of the temporal overlap by monitoring the mulit-photon photoemission yield of the pump beam and the driver of the HHG in spatial overlap (b) while scanning the delay $(\Delta t)$ between the two beams. (b) Real-space image of both beams on the chessy test specimen to optimize the spatial overlap. A field aperture is used (dashed black line) to isolate the region of good spatial overlap. The inset shows only the driver of the HHG. 


\section{B.3. Calibration of the static photoemission spectroscopy data}

\section{B.3.1. Energy calibration}

The energy calibration has been performed like described in section 3.3.2, i.e., measurements with different sample voltages were performed $\left(U_{\text {Sample }}=19 \mathrm{~V}, 20 \mathrm{~V}, 21\right.$ $\mathrm{V}, 22 \mathrm{~V})$. The conversion from time-of-flight $\mathrm{t}$ to the corresponding energies $E$ has been found by matching characteristic features in the spectra as shown in figure B.3. the resulting parameters are shown in table B.1.
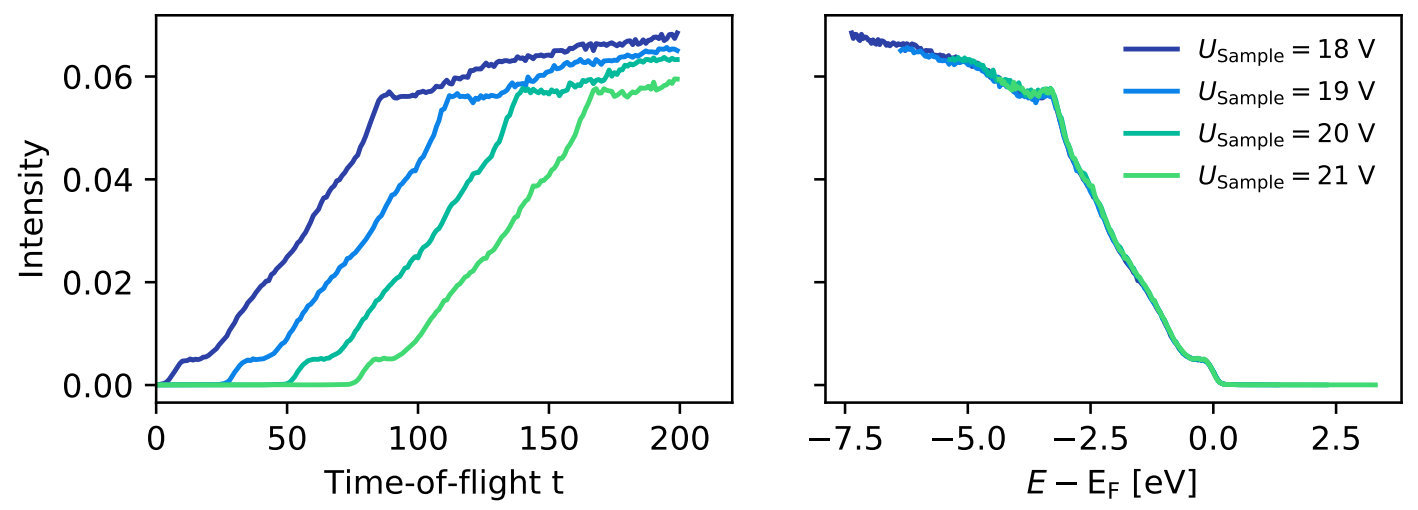

Figure B.3.: (Left) Raw spectra integrated over the full accessible $k_{x}, k_{y}$ range for different sample voltages. Each temporal pixel corresponds to a time window of 27.5 ps. (Right) Spectra for the different sample voltages after performing the conversion to kinetic energies using the parameters in table B.1. All spectra lie reasonably well on top of each other, showing that a good conversion has been found. 


\begin{tabular}{|c|c|}
\hline$a_{0}$ & $-0.0439 \mathrm{eV}$ \\
\hline$a_{1}$ & $0.0052 \mathrm{eV}^{1 / 2}$ \\
\hline$a_{2}$ & $0 \mathrm{eV}^{1 / 3}$ \\
\hline $\mathrm{E}_{\mathrm{F}}$ & $-18.70 \mathrm{eV}$ \\
\hline
\end{tabular}

Table B.1.: Table showing the parameters $\left(a_{0}, a_{1}, a_{2}, \mathrm{E}_{\mathrm{F}}\right)$ used for the energy calibration. The conversion from time-of-flight t to kinetic energies $E$ using these parameters can be seen in figure B.3.

\section{B.3.2. Symmetrization of the momentum maps}

The measured 4D data sets from graphene have been symmetrized to it's known hexagonal symmetry by a set of rotation, stretching and shearing matrices [269].

$$
\begin{gathered}
\mathrm{R}(\alpha)=\left(\begin{array}{ccc}
\cos (\alpha) & \sin (\alpha) & 0 \\
-\sin (\alpha) & \cos (\alpha) & 0 \\
0 & 0 & 1
\end{array}\right) \\
\mathrm{S}\left(x_{\text {Stretch }}, y_{\text {Stretch }}\right)=\left(\begin{array}{ccc}
x_{\text {Stretch }} & 0 & 0 \\
0 & y_{\text {Stretch }} & 0 \\
0 & 0 & 0
\end{array}\right) \\
\mathrm{Z}\left(x_{\text {Shear }}, y_{\text {Shear }}\right)=\left(\begin{array}{ccc}
0 & x_{\text {Shear }} & 0 \\
y_{\text {Shear }} & 0 & 0 \\
0 & 0 & 0
\end{array}\right)
\end{gathered}
$$

This has been performed by David Schmitt and is briefly introduced here. At first, the measured momentum map at the Dirac point energy $E-\mathrm{E}_{\mathrm{F}}=-0.4 \mathrm{eV}$ is taken and smoothed by a Gaussian filter. Subsequently the six Dirac points positions ${ }^{1}$ $\left(x_{i}, y_{i}, i=0 \ldots 5\right)$ are found by using a peak determination routine based on the DAOFIND algorithm [361, 362]. The hexagonal symmetry restricts the distances $\overline{\Gamma K}=\overline{\Gamma K^{\prime}}=\overline{K^{\prime} \mathrm{K}}$, which can expressed by cost function $G\left(x_{0}, y_{0}, \ldots, x_{5}, y_{5}, x_{c}, y_{c}\right)$ :

$$
\begin{aligned}
G\left(x_{0}, y_{0}, \ldots, x_{5}, y_{5}, x_{c}, y_{c}\right) & =\sqrt{\sum_{i=0}^{5}\left(d\left(x_{i}, y_{i}\right)-m_{d}\right)^{2}} / m_{d} \\
& +\sqrt{\sum_{i=0}^{5}\left(d_{c}\left(x_{i}, y_{i}, x_{x}, y_{c}\right)-m_{d_{c}}\right)^{2} / m_{d_{c}}}
\end{aligned}
$$

with the distances between the Dirac point positions $d\left(x_{i}, y_{i}\right)$ :

$$
d\left(x_{i}, y_{i}\right)=\left|\left(x_{(i+1) \bmod 6}-x_{i}\right)^{2}+\left(y_{(i+1) \bmod 6}-y_{i}\right)^{2}\right|,
$$

\footnotetext{
1 Similarly, the six replicas around one $K, K^{\prime}$ point can be used in a measurement with a higher magnification, focussing on one Dirac point.
} 
their mean $m_{d}$ :

$$
m_{d}=\frac{\sum_{i=0}^{5}\left(d\left(x_{i}, y_{i}\right)\right.}{6}
$$

the distances between the Dirac point positions $\left(x_{i}, y_{i}\right)$ and the $\Gamma$ point position $\left(x_{c}, y_{c}\right)$ :

$$
d_{c}\left(x_{i}, y_{i}, x_{c}, y_{c}\right)=\left|\left(x_{i}-x_{c}\right)^{2}+\left(y_{i}-y_{c}\right)^{2}\right|
$$

and their mean:

$$
m_{d_{c}}=\frac{\sum_{i=0}^{5}\left(d_{c}\left(x_{i}, y_{i}, x_{c}, y_{c}\right)\right.}{6} .
$$

The cost function is calculated for discretized steps of $\alpha,\left(x_{\text {Stretch }}, y_{\text {Stretch }}\right),\left(x_{\text {Shear }}, y_{\text {Shear }}\right)$ whereby it's minimum yields the best paramter set to fulfil the hexagonal symmetry, which is subsequently used to symmetrize all measurements in the $4 \mathrm{D}$ data set. The raw measurement and the symmetrized data set is shown exemplary in figure B.4.

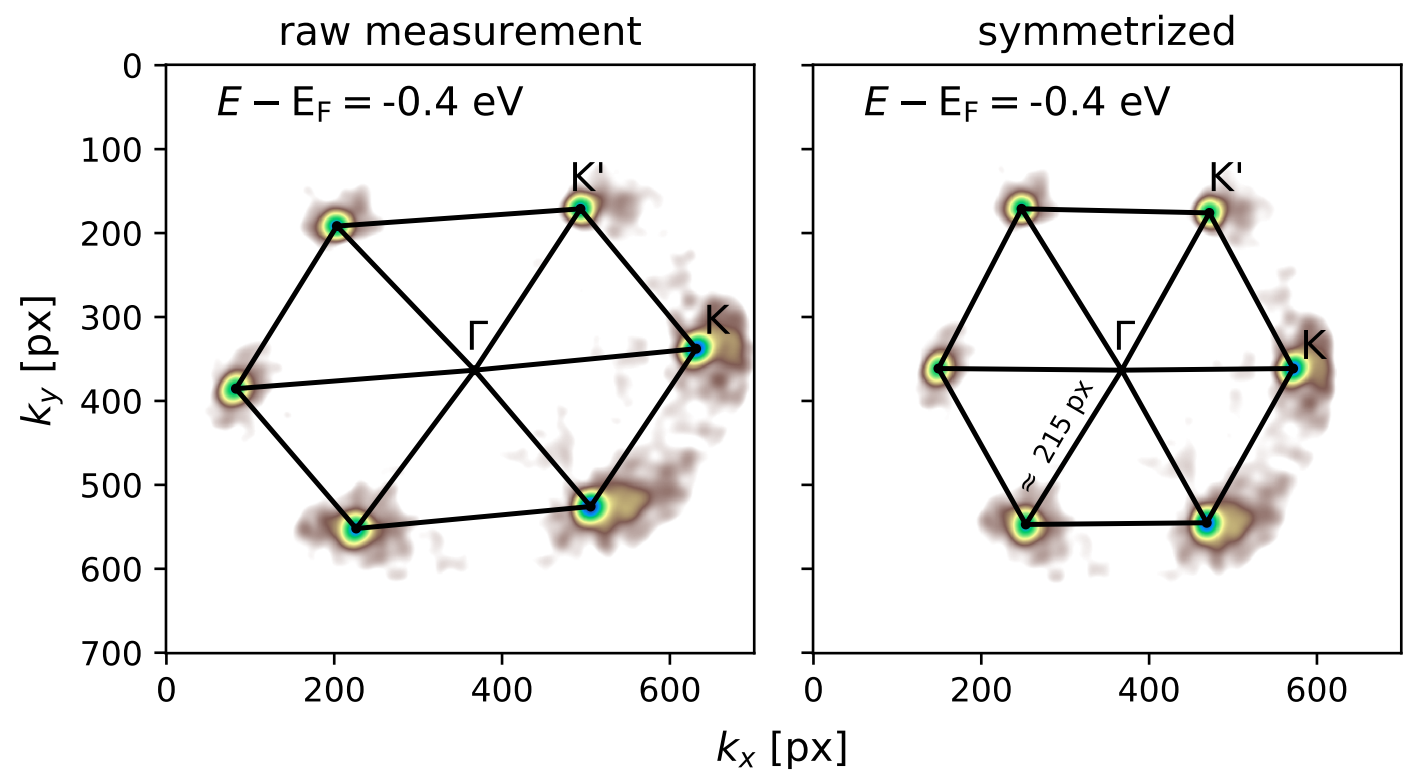

Figure B.4.: Momentum maps taken at $E-\mathrm{E}_{\mathrm{F}}=-0.4 \mathrm{eV}$ to visualize the outcome of the symmetrization procedure. The positions of the $\mathrm{K}$ and $\mathrm{K}^{\prime}$ points, determined by the peak determination routine (black dots), as well as the $\Gamma$ point are marked. The raw measurement (left) shows a distorted symmetry, as evidenced by the different distances $\overline{\Gamma K} \neq \overline{\Gamma K^{\prime}} \neq \overline{\mathrm{K}^{\prime} \mathrm{K}}$. After symmetrization (right), the dirac points show the hexagonal symmetry as expected. 


\section{B.3.3. Momentum calibration}

The momentum calibration for the symmetrized 4D data set is performed according to section 3.3.3. The distances $\overline{\Gamma \mathrm{K}}=\overline{\Gamma \mathrm{K}^{\prime}}=\overline{\mathrm{K}^{\prime} \mathrm{K}}$ can be gained directly from the symmetrization procedure to be $\approx 215 \mathrm{px}$ (see figure B.4), which results in a conversion factor $\kappa=0.0079 \AA^{-1} /$ px with the known distances $\overline{\Gamma K}=\overline{\Gamma K^{\prime}}=\overline{\mathrm{K}^{\prime} \mathrm{K}}=$ $\frac{4 \pi}{3 \sqrt{3} a}$ and a carbon-carbon distance of $a \approx 1.42 \AA[162-164$.

\section{B.4. Calibration of the time-resolved photoemission spectroscopy data}

\section{B.4.1. Energy calibration}

The energy calibration has been performed like described in section 3.3.2, i.e., measurements with different sample voltages were performed $\left(U_{\text {Sample }}=18 \mathrm{~V}, 19 \mathrm{~V}, 20\right.$ $\mathrm{V}, 21 \mathrm{~V}, 22 \mathrm{~V})$. The conversion from time-of-flight t to the corresponding energies $E$ has been found by matching characteristic features in the spectra as shown in figure B.5, the resulting parameters are shown in table B.2.
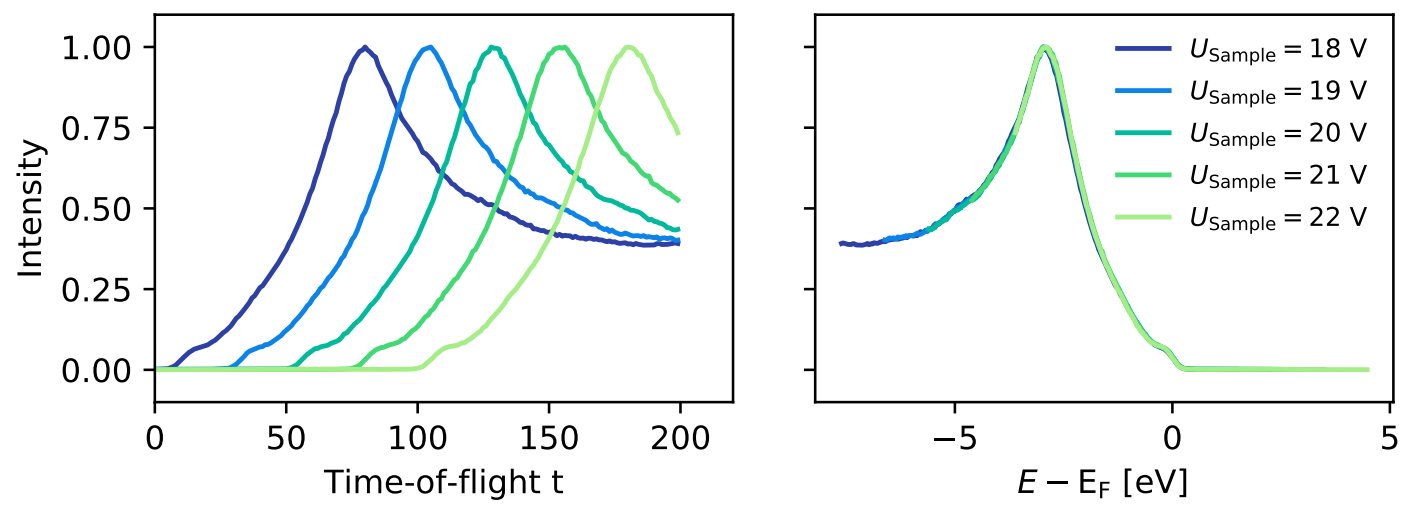

Figure B.5.: (Left) Raw spectra integrated over the full accessible $k_{x}, k_{y}$ range for different sample voltages. Each temporal pixel corresponds to a time window of 27.5 ps. (Right) Spectra for the different sample voltages after performing the conversion to kinetic energies using the parameters in table B.2. All spectra lie reasonably well on top of each other, showing that a good conversion has been found. Note that small intensity differences between the different measurements can be observed due to fluctuations in the HHG yield. The corresponding Fermi-Dirac-fit is shown in fig. B.7 and is also used to estimate the energy resolution of the measurement. 


\begin{tabular}{|c|c|}
\hline$a_{0}$ & $-0.0443 \mathrm{eV}$ \\
\hline$a_{1}$ & $0.0043 \mathrm{eV}^{1 / 2}$ \\
\hline$a_{2}$ & $0 \mathrm{eV}^{1 / 3}$ \\
\hline $\mathrm{E}_{\mathrm{F}}$ & $-17.58 \mathrm{eV}$ \\
\hline
\end{tabular}

Table B.2.: Table showing the parameters $\left(a_{0}, a_{1}, a_{2}, \mathrm{E}_{\mathrm{F}}\right)$ used for the energy calibration. The conversion from time-of-flight t to kinetic energies $E$ using these parameters can be seen in figure B.5.

\section{B.4.2. Symmetrization of the momentum maps}

The measured $4 \mathrm{D}$ data sets for a single Dirac cone have been symmetrized like described above, whereas now the six replicas around each dirac cone are used to enforce the hexagonal symmetry. These replicas (compare figure 2.11) result from the interfacial $\left(6 \sqrt{3} \times 6 \sqrt{3} \mathrm{R} 30^{\circ}\right)$ reconstruction between the $\mathrm{SiC}(0001)$ substrate and the graphene layer [172, 274, 363 368], whereby a carbon-rich buffer layer is formed in between them. The definitive explanation of the replicas as an intial- [367, 369] or final state [364] effect in photoemission is still an unresolved question [364] and won't be discussed further within this thesis. The measured- and symmetrized momentum maps at the dirac point energy are shown in figure B.6. 


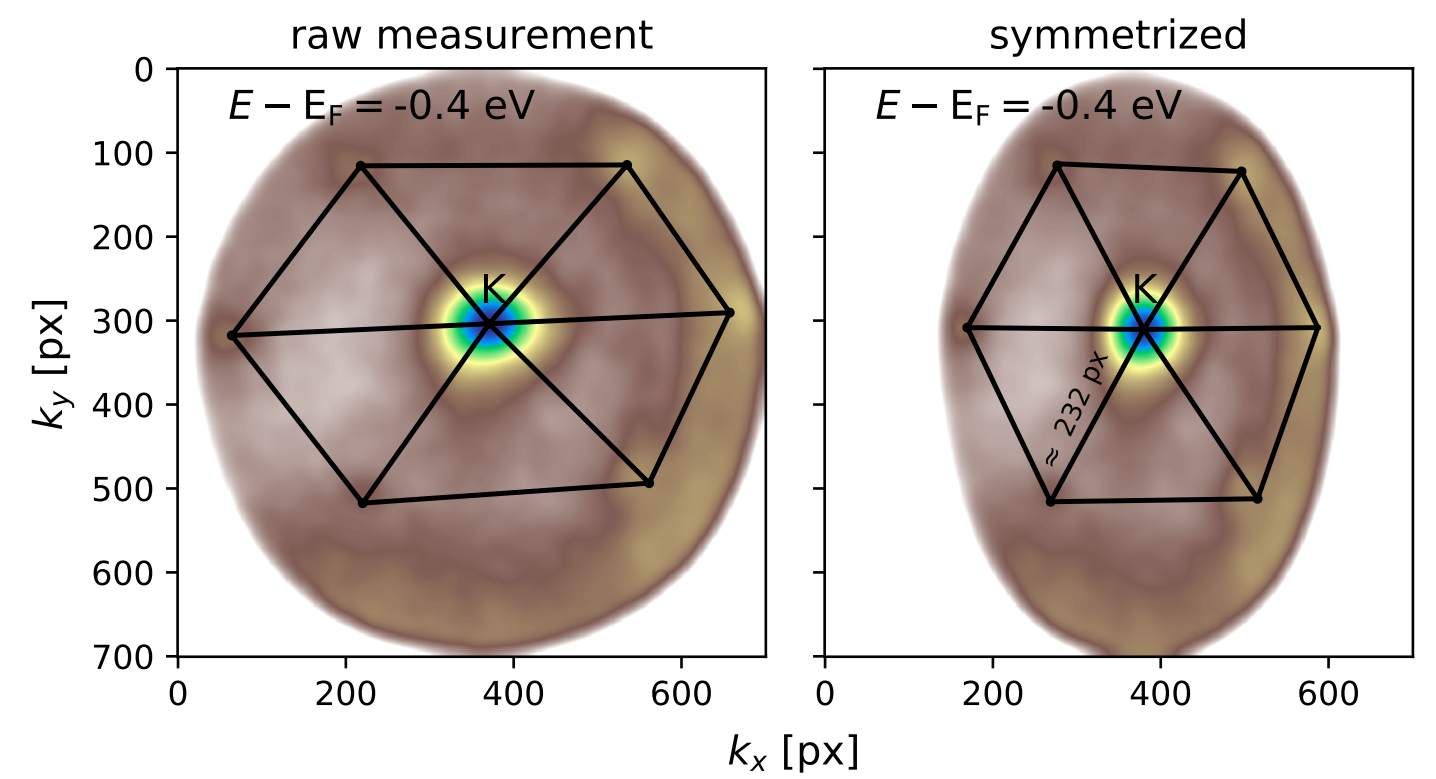

Figure B.6.: Momentum maps taken at $E-\mathrm{E}_{\mathrm{F}}=-0.4 \mathrm{eV}$ to visualize the outcome of the symmetrization procedure. The position of the $\mathrm{K}$ point and the replicas, determined by the peak determination routine (black dots) are marked. The raw measurement (left) shows a distorted symmetry. After symmetrization (right), the replica show the hexagonal symmetry as expected.

\section{B.4.3. Momentum calibration}

Similarly, the momentum calibration for the symmetrized 4D data set can be performed using the distance of the six replicas towards the $\mathrm{K}$ point which measures $\approx 232 \mathrm{px}$. The conversion factor can therefore be calculated to $\kappa=0.0019 \AA^{-1} / \mathrm{px}$ with the known distance of the replicas $\approx 0.4305 \AA^{-1}$ (resulting from the $6 \sqrt{3} \mathrm{x}$ $6 \sqrt{3} \mathrm{R} 30^{\circ}$ reconstruction).

\section{B.4.4. Normalization}

The normalization has been performed according to section 3.3.5 with a normalization energy of $E_{\text {norm }}=-4.9 \mathrm{eV}$ resulting in a clear time trace for the generated sideband, which can be connected to the pump pulse shape (see figure 4.5).

\section{Energy resolution}

The energy resolution of the experiment can be estimated from the data obtained out of temporal overlap (shown in B.7 (a), $\Delta t=-200$ fs) by fitting a Fermi-Dirac 
distribution $(300 \mathrm{~K})$ convoluted with a Gaussian broadening to the measured Fermi edge (shown in B.7 (b)). The integration area used to extract the spectrum from the measured data, as well as, the upper and lower limit used in the fit procedure area pictured. The energy resolution is commonly defined as the FWHM of the Gaussian broadening which results in FWHM $\approx 220 \mathrm{meV}$ for this experiment. As described in the main text this energy resolutionis related to the bandwidth of the EUV probe and the energy resolution of the momentum microscope (mostly defined by the time-of-flight (ToF) voltage).

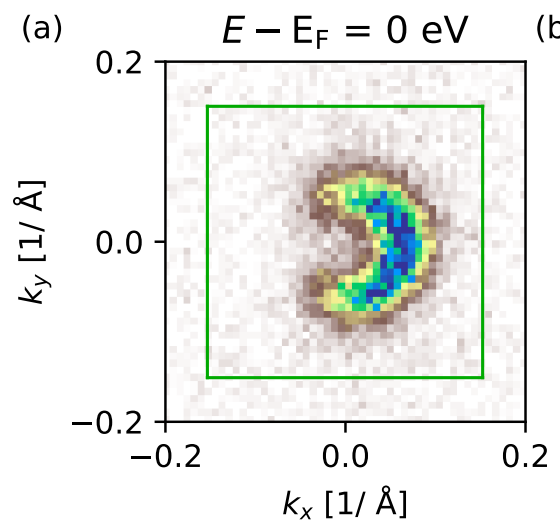

(b)

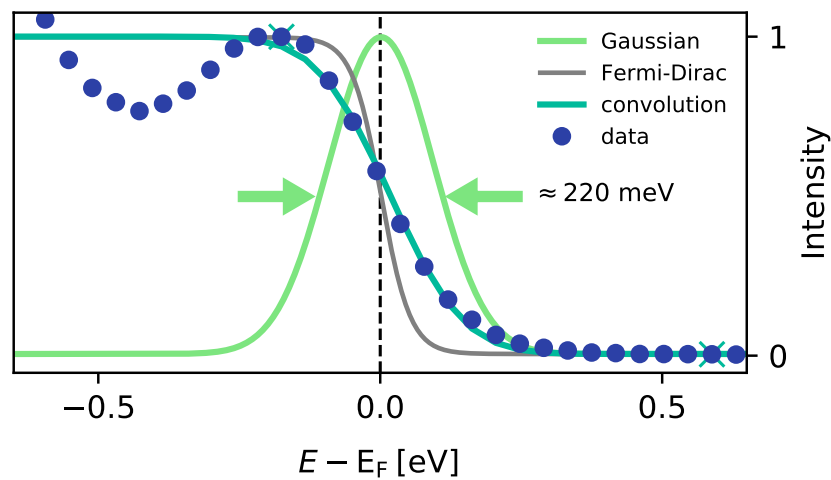

Figure B.7.: (a) Momentum cut at $E-\mathrm{E}_{\mathrm{F}}=0 \mathrm{eV}$ out of temporal overlap $(\Delta t=-200$ fs) showing the integration area in $k_{x}, k_{y}$ which was used to estimate the energy resolution of the experiment. (b) The energy resolution is extracted by fitting the convolution of a Fermi-Dirac distribution (300 $\mathrm{K})$ and a Gaussian broadening to measured Fermi edge $(\Delta t=-200$ fs, integration area shown in (a)). The upper and lower limit of the datapoints which were used to fit the convolution are marked with an ' $\mathrm{X}$ '. The FWHM of the Gaussian broadening is $\approx 220 \mathrm{meV}$. 


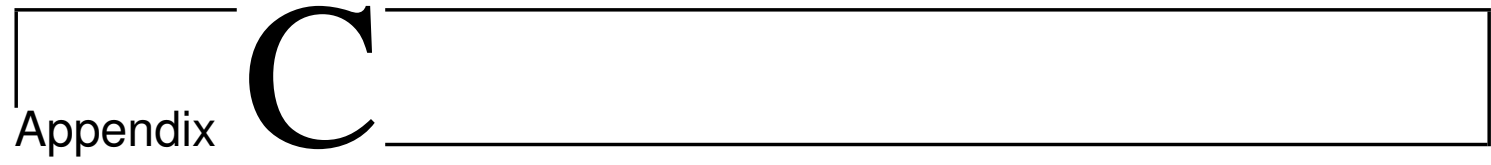

\section{Supplementary information for chapter 5}

\section{C.1. s-polarized measurement}

\section{C.1.1. Calibration}

The calibration has been performed similar to section B.4 and the respective constants are shown in table C.1.

\begin{tabular}{|c|c|}
\hline$a_{0}$ & $-0.0449 \mathrm{eV}$ \\
\hline$a_{1}$ & $0.0043 \mathrm{eV}^{1 / 2}$ \\
\hline$a_{2}$ & $0 \mathrm{eV}^{1 / 3}$ \\
\hline $\mathrm{E}_{\mathrm{F}}$ & $-17.15 \mathrm{eV}$ \\
\hline$\kappa$ & $0.0019 \AA^{-1} / \mathrm{px}$ \\
\hline$E_{\text {Norm }}$ & $-2 \mathrm{eV}$ \\
\hline
\end{tabular}

Table C.1.: Table showing the parameters $\left(a_{0}, a_{1}, a_{2}, \mathrm{E}_{\mathrm{F}}\right.$ and $\left.\kappa\right)$ used for the calibration.

\section{C.2. Data normalization and maxima determination}

The timetraces obtained from the colored regions in figure 5.3 (a) and figure 5.4 (a) have been normalized to their respective minimum and maximum value within the whole tr- measurement, i.e., their intensity is normalized from 0 to 1 . The respective maxima positions have been determined by fitting a Gaussian (solid line) to the centred peak of the timetrace in the data range from -20 to $+30 \mathrm{fs}$. This is shown in figure C.1. Additionally, an increased levelling of the data around the temporal overlap $(\Delta t=0 \mathrm{fs})$ can be seen in the measurement with the s-polarized pump beam, which can be understood as state blocking (Pauli-blocking) due to higher fluency [216] and results in a less precise determination of $t_{\max }$ (as evidenced by the errorbars). 

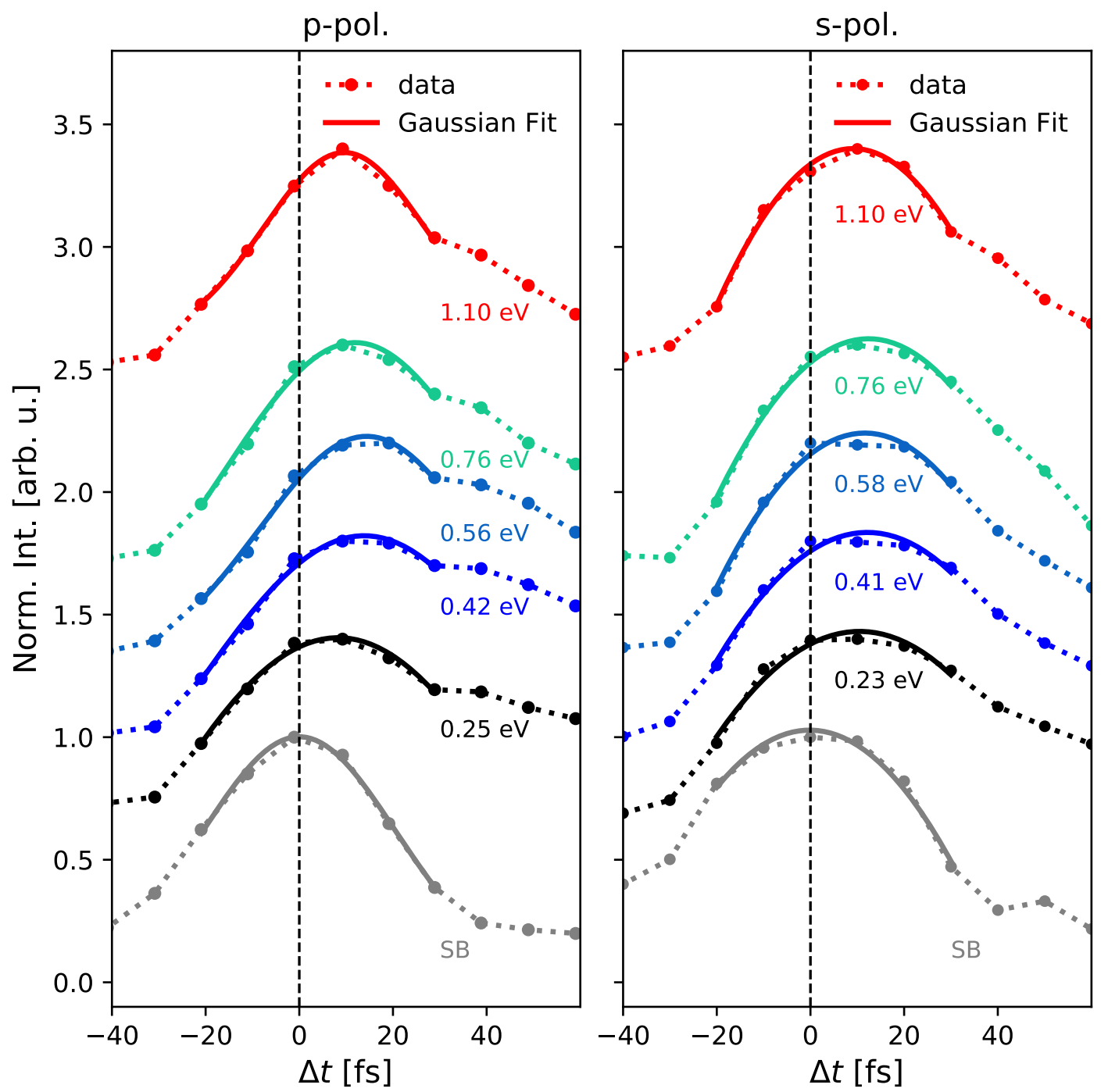

Figure C.1.: Timetraces for p-pol. (left) and s-pol. (right) for the different marked regions in the respective figures 5.3 and 5.4 . The maxima positions are determined by a Gaussian fit (solid line) to the data (dots) in the time interval from $\Delta t=-20$ to +30 fs. For clarity, all spectra are shifted along the $y$-axis in accordance to their energetic distance. One can notice, that the fitting procedure works better in the p-polarized case, since there a clear peak can be observed, whereas in the s-polarized measurement a levelling is seen, which results in a less clear maxima determination (evidenced by the errorbars in figure 5.4). 


\section{C.3. Influence of the post-pulse to the observed dynamics}

The post-pulse, which was already observed in chapter 4 , is also present in the temporal dynamics of the hot charge-carriers but does not influence their scattering behaviour closely around the temporal overlap. This can be evidenced from the thermal energy of the carriers shown in C.2 (b), which is determined by fitting hot Fermi-Dirac distributions to the spectra obtained in the direction of photoexcitation for the p-polarized pump beam (using the same integration areas as in figure 5.3. The spectra for selected time-delays (data points) together with their hot FermiDirac fits are shown in C.2 (a), whereby the inset shows the high energetic tail of the distribution (0.7-1.3 eV). One can directly observe that the carriers are not nicely described by a hot Fermi-Dirac distribution closely around the temporal overlap $(\Delta t=0 \mathrm{fs})$ from the increasing error in the thermal energy ${ }^{1}$ in (b) and the drop in the coefficient of determination $R^{2}$ of the fit (inset in (b)). The post-pulse is evidenced by an additional increase in thermal energy closely centred around $60-70$ fs together with a drop in the coefficient of determination $R^{2}$. From their width and from the FROG measurement (see section 3.1.2) one can estimate that the dynamics around the temporal overlap up to +30 fs are not strongly influenced by the postpulse. On longer timescales $\geq 100$ fs the carriers are again nicely described by a hot Fermi-Dirac distribution and one can follow the cooling of the system.

1 Obviously, the thermal energy is not nicely defined if the electrons are not described by a hot Fermi-Dirac distribution. 

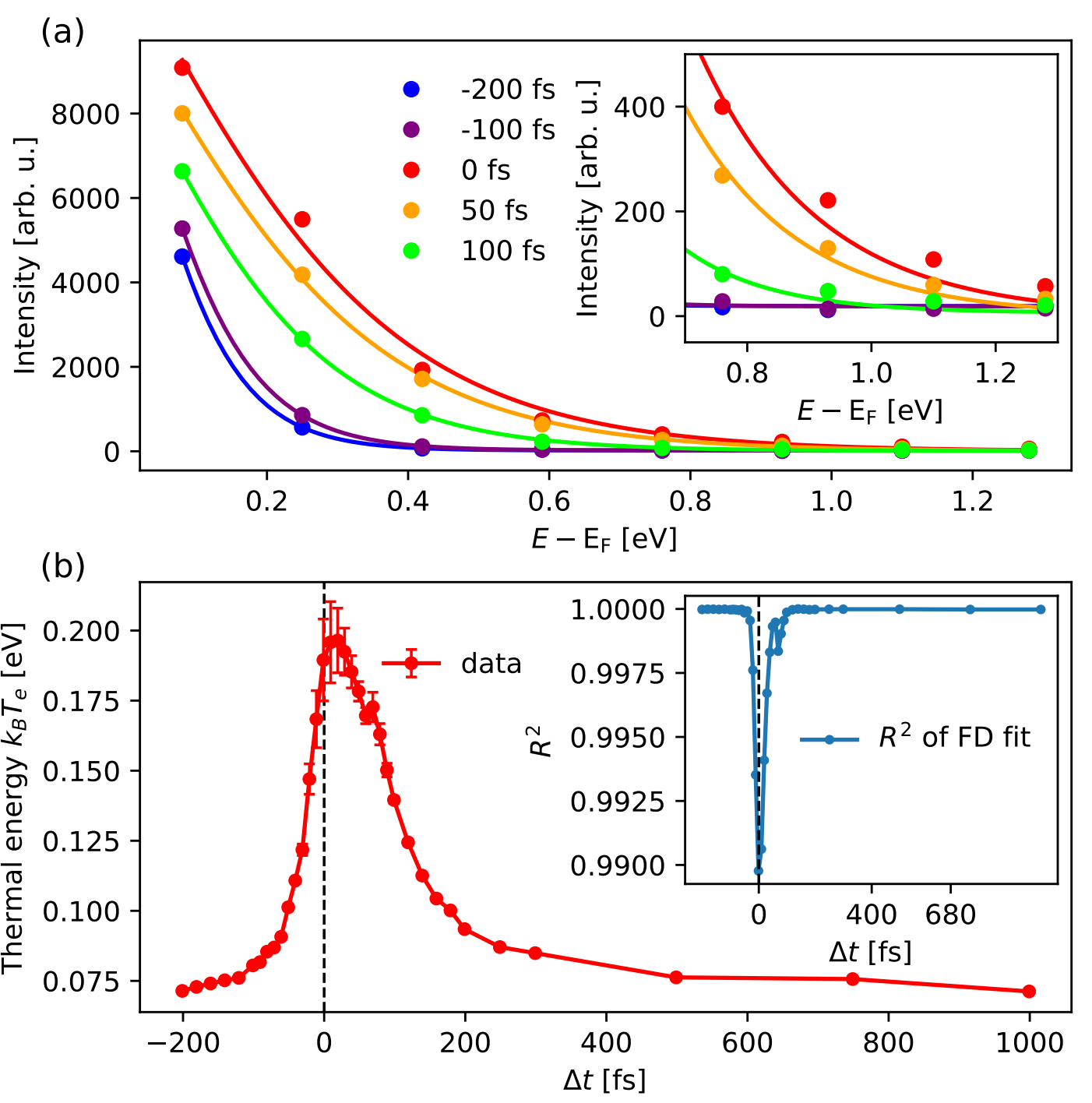

Figure C.2.: (a) Spectrum of the charge carriers in the direction of photoexcitation for the p-pol. measurement for selected time delays (using the same integration areas as in figure 5.3), together with the fitted hot FermiDirac distributions. The inset shows a close up of the high energetic tail of the distribution. (b) Thermal energy as estimated from the FermiDirac fit as a function of delay $(\Delta t)$. Around the temporal overlap the distribution does not follow a hot Fermi-Dirac distribution as evidenced by the errorbars and the drop in the coefficient of determination $R^{2}$ (inset). The post-pulse arriving at a time delay of $60-70 \mathrm{fs}$ results in an additional increase of thermal energy and a drop in the $R^{2}$ value. 


\section{C.4. Details on the simulation}

In the following details on the simulations performed by Marius Weber and HansChristian Schneider are presented.

\section{C.4.1. Boltzmann Scattering}

The general treatment of electron-electron scattering for a solid in the single particle picture is outlined here. The time evolution of the electronic distribution functions $f_{\boldsymbol{k}}^{\nu}$ in state $|\nu, \boldsymbol{k}\rangle$ is calculated, where $\nu$ is the band index, by using the Boltzmann scattering integral [370]

$$
\begin{aligned}
\frac{d}{d t} f_{\boldsymbol{k}}(t)= & \frac{2 L^{2 n}}{\hbar^{2}(2 \pi)^{n}} \int d^{n} q \int d^{n} l\left[V_{\mu_{2}, \mu_{3}}^{\nu, \mu_{1}}(\boldsymbol{q})\right]^{2} \Upsilon(\Delta E, t, \Gamma) \\
& {\left[1-f_{\boldsymbol{k}}^{\nu}\right]\left[1-f_{\boldsymbol{l}+\boldsymbol{q}}^{\mu_{1}}\right] f_{\boldsymbol{l}}^{\mu_{2}} f_{\boldsymbol{k}+\boldsymbol{q}}^{\mu_{3}}-f_{\boldsymbol{k}}^{\nu} f_{\boldsymbol{l}+\boldsymbol{q}}^{\mu_{1}}\left[1-f_{\boldsymbol{l}}^{\mu_{2}}\right]\left[1-f_{\boldsymbol{k}+\boldsymbol{q}}^{\mu_{3}}\right] }
\end{aligned}
$$

which describes the two-fermion scattering transition $\boldsymbol{l} \rightarrow \boldsymbol{l}+\boldsymbol{q}$ and $\boldsymbol{k}+\boldsymbol{q} \rightarrow \boldsymbol{k}$ mediated by the screened Coulomb interaction. The energy difference $\Delta E$ between initial and final states enters here via

$\Upsilon(\Delta E, t, \Gamma)=\frac{\hbar}{\Gamma^{2}+\Delta E^{2}}\left(\left[\Delta E \sin \left(\frac{\Delta E}{\hbar}\left(t-t_{0}\right)\right)-\Gamma \cos \left(\frac{\Delta E}{\hbar}\left(t-t_{0}\right)\right)\right] e^{-\frac{\Gamma}{\hbar}\left(t-t_{0}\right)}+\Gamma\right)$.

where $\Gamma$ is the imaginary part of the self-energy. This expression approaches $\Upsilon \rightarrow$ $\hbar \delta(\Delta E)$ at longer times and for $\Gamma \rightarrow 0$, which conserves kinetic energy, but at short times it leads to an increase of the kinetic energy of our system during the building up of correlations [371. In order to have a computationally feasible model, the self energy will be assumed as constant $\Gamma$. A finite constant $\Gamma$ also leads to an increase in energy and we choose $\Gamma=0.001 \mathrm{meV}$ to keep this effect small for the duration of our simulations. Equation (C.1) can be derived from a self-consistent quantum kinetic Boltzmann equation by integrating over retardation effects [370, 372].

\section{C.4.2. Graphene}

For the numerical solution of the dynamical equation for the distribution some simplifications are employed, which are described below. The bandstructure is linearized around the $\Gamma$ point [373] with a slope of $650 \mathrm{meV} / \mathrm{nm}$. The matrix elements are calculated using the linearized dispersions. The Coulomb matrix elements can then be written as:

$$
V_{\mu_{2}, \mu_{3}}^{\nu, \mu_{1}}=V_{q}^{2 D} g_{\mu_{2}, \mu_{3}}^{\nu, \mu_{1}}
$$

and a statically screened Coulomb potential $V_{q}^{2 D}$ is used. It is assumed that an additional momentum dependent factor in Eq. (C.3) can be dropped, 373. A background dielectric constant of 4 is used and a constant screening parameter of 
$\kappa=4 \mathrm{~nm}^{-1}$. The coefficient $g_{\mu_{2}, \mu_{3}}^{\nu, \mu_{1}}$ is determined by

$$
g_{\mu_{2}, \mu_{3}}^{\nu, \mu_{1}}=\frac{1}{4}\left(1+c_{\nu, \mu_{2}} \frac{e\left(\boldsymbol{k}_{1}\right)^{*} e\left(\boldsymbol{k}_{3}\right)}{\left|e\left(\boldsymbol{k}_{1}\right) e\left(\boldsymbol{k}_{3}\right)\right|}\right)\left(1+c_{\mu_{1}, \mu_{3}} \frac{e\left(\boldsymbol{k}_{2}\right)^{*} e\left(\boldsymbol{k}_{4}\right)}{\left|e\left(\boldsymbol{k}_{2}\right) e\left(\boldsymbol{k}_{4}\right)\right|}\right) \text {. }
$$

The prefactor $c_{a, b}$ equals 1 for $a=b$ (intraband processes) and -1 for $a \neq b$ (interband processes), further $e(\boldsymbol{k})=-\frac{a_{0} \sqrt{3}}{2}\left(i k^{x}+k^{y}\right)$. These matrix elements describe all scattering channels near the Dirac cone. To quantify the impact of Auger processes we are interested in index combinations $\mu_{1}=\mu_{2}=\mu_{3} \neq \nu$ and all their permutations. Within these eight possible combinations, Auger recombination and impact excitation are included, both types of Auger scattering having basically the same strength.

Two further important simplifications were made: In order to focus on electronelectron scattering, electron-phonon scattering processes are not included, which would result in a decrease of the kinetic energy. The excitation is included in a purely phenomenological way by instantaneously creating the excited carrier density.

\section{C.4.3. Numerical Details}

Finally, a short review of the used numerical scheme and the boundary conditions is provided. Eq. (C.1) is implemented for a Cartesian $k$ grid, which has the advantage that it does not introduce numerical errors in the carrier density conservation. Therefore, we are able to calculate the scattering properties of the complete 2D k-space with an arbitrary excitation. Due to the numerical costly right-hand side of Eq.C.1) we used a parallelization techniques and and a Dormand-Prince differential equation solver with an adaptive step size. Finally the k-space was sampled with 51 grid points in each direction and with a maximal momentum of $k_{\max }=2.5 \mathrm{~nm}^{-1}$. This approach using a Cartesian $k$ grid conserves carrier density by construction, which is important as we are looking relatively small effects at high energies $(>800$ $\mathrm{meV})$. On the other hand, it is numerically quite costly. The initial distribution is chosen as a Fermi-Dirac distribution $f_{ \pm}^{\text {eq }}=1 /\left(1+e^{\left[\left(E_{ \pm}-\mu\right) /\left(k_{\mathrm{B}} T\right)\right]}\right)$ with a chemical potential of $400 \mathrm{meV}$ to model the relaxed electrons introduced by doping.

An instantaneous excitation is assumed of the form

$$
\delta f=A e^{-\frac{1}{b}\left(\boldsymbol{k}-\boldsymbol{k}_{e}\right)^{2}}\left|\sin \left(k_{x}\right)\right|
$$

with the amplitude $A$, the width of the excitation $b$ and the center of the excitation $\boldsymbol{k}_{e}$, which follows the spacial shape of the measured excitation and the calculations from Ref. [234]. In order to distinguish between scattering effects and building up of correlations, we let the system with the doped carrier density relax and then initialize the calculation by changing the electronic distributions by $\pm \delta f$ in the upper/lower band with positive/negative energy dispersion.

To eliminate Auger type processes, the Coulomb matrix elements relevant for these transitions are excluded by setting $g_{\mu, \mu}^{\mu, \nu}=0$. Thus the scattering dynamics with and 


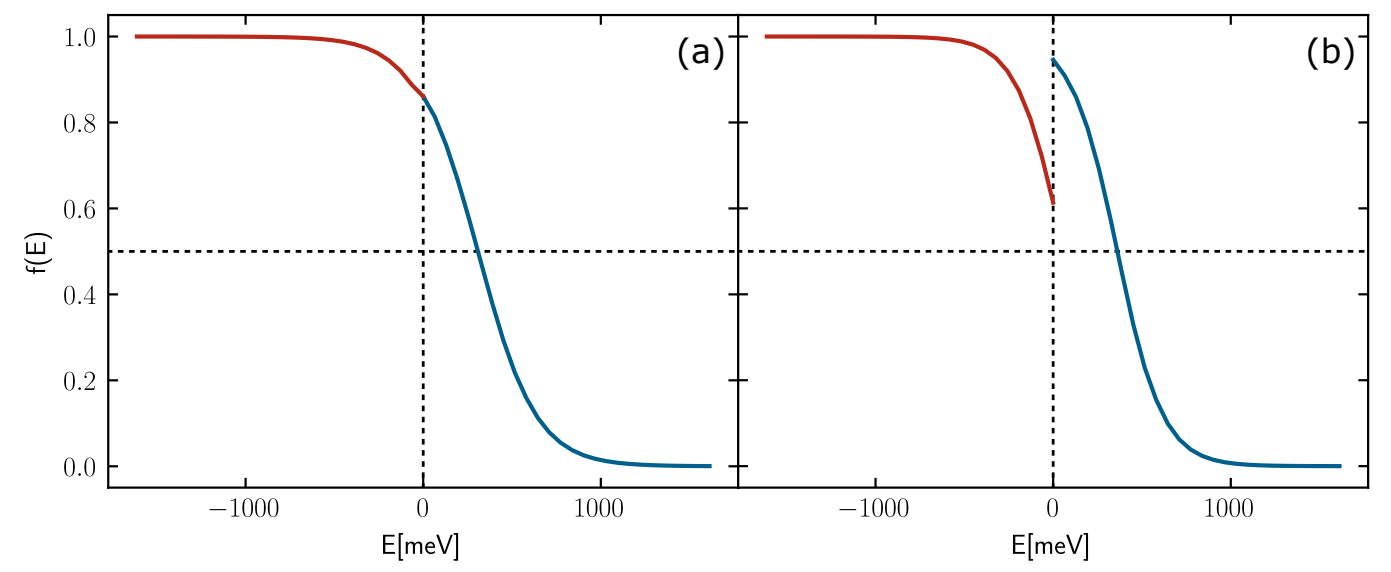

Figure C.3.: Distribution functions after $300 \mathrm{fs}$, for the full calculation (a), and the calculation without Auger processes. The red/blue part corresponds to the lower/upper band. The full calculation reaches a quasi-equilibrium with a chemical potential of about $300 \mathrm{meV}$. Neglecting Auger processes in the calculation prevents the system from reaching a quasi equilibrium. Taken from [80].

without Auger processes can be compared. In order to replicate the photoemission experiments to some extent, we integrate the time-dependent distributions over the $\left(0, k_{y}\right)$ axis for the energy interval $0.8-1.3 \mathrm{eV}$ to obtain the time dependent density traces shown in Fig. 4 in the main text. A numerical test case of the dynamics is presented in Fig. C.3, where the energy-dependent distributions obtained $300 \mathrm{fs}$ after the instantaneous excitation are compared. If all scattering processes are included the system reaches a Fermi-Dirac distribution with a higher temperature and a different chemical potential, as the excitation increases the kinetic energy of carriers, and electron-electron scattering does not dissipate this energy. Switching the Auger scattering contributions off leads to the distributions in Fig. C.3(b), which are clearly not equilibrated. 


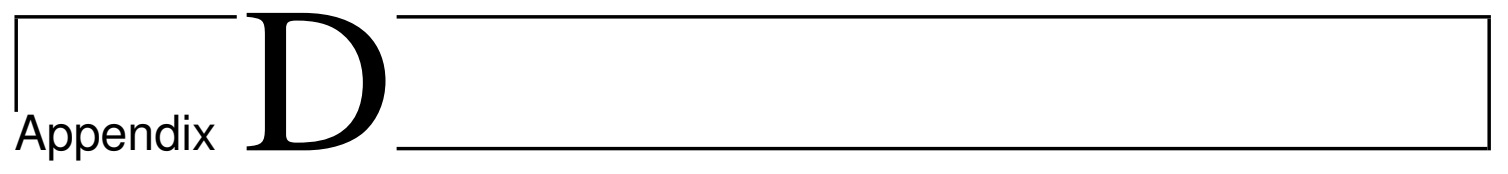

\section{Supplementary information for chapter 6}

\section{D.1. Raw-data post processing}

\section{D.1.1. Calibration of the energy axis}

The energy calibration for the $\mathrm{Au}(111)$ measurement is shown in section 3.3.2. The conversion from time-of-flight t to the corresponding energies $E$ has been found by matching characteristic features in the spectra, the resulting parameters are shown in table 3.1 .

\section{D.1.2. Momentum calibration}

The momentum calibration has been performed exemplary in section 3.3 .3 .

\section{Normalization}

The normalization has been performed according to section 3.3.5 with a normalization energy of $E_{\text {norm }}=-2.4 \mathrm{eV}$ resulting in a clear time trace for the generated sideband (see Fig. D.2.

\section{D.1.3. Shift-correction of the momentum maps}

A small momentum shift between the occupied bandstructure $\mathrm{n}_{0}$ and the generated sideband $\mathrm{n}_{1}$, induced by a non-perfect alignment of the momentum microscope, was post-corrected by assigning the $\bar{\Gamma}$-point in the middle of the SS and it's replica and linearly interpolating this momentum shift in energy. Selected corrected and uncorrected ARPES spectra $\left(E, k_{y}\right)$ are shown in figure D.1 as a comparison. This correction was applied to all 3D data sets in the corresponding measurement. 


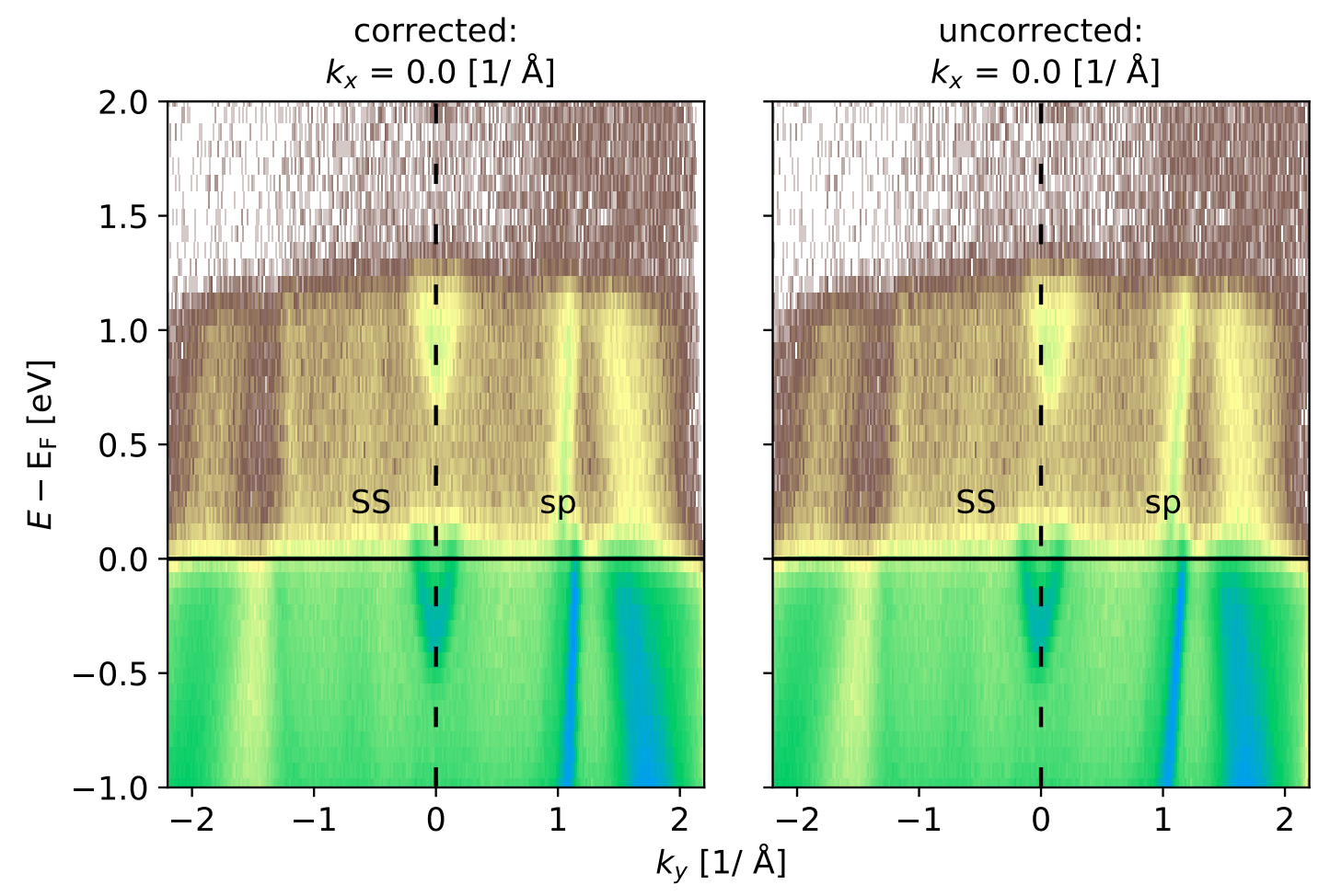

Figure D.1.: Corrected (left) and uncorrected (right) ARPES spectra obtained with the nearly p-pol. driver in temporal overlap $(\Delta t=0 \mathrm{fs})$. The uncorrected image shows a clear momentum shift, which was post-corrected as described in the text. The $\left(E, k_{y}\right)$-resolved spectra are retrieved by slicing the experimentally obtained three-dimensional data set in $k_{y}$ direction and integrating over a $0.13 \AA^{-1}$ large region in $k_{x}$ direction. The surface state (SS) and the sp-band transition are marked in the spectra. Taken from [79].

\section{D.2. Time- and energy resolution}

The setup facilitates the real-time measurement of the temporal evolution of the ultrafast charge carrier dynamics with full energy and in-plane momentum resolution [78]. This is evident in Fig. D.2 where exemplary $\left(E, k_{y}\right)$ and $\left(k_{x}, k_{y}\right)$ cuts are shown for various delays $(\Delta t)$ of the driving IR and the probing EUV pulse. By scanning the delay, $\Delta t=0 \mathrm{fs}$ is determined at the position where maximum sideband intensity is observed, similar as done in Ref. [78, 95]. 
(a)

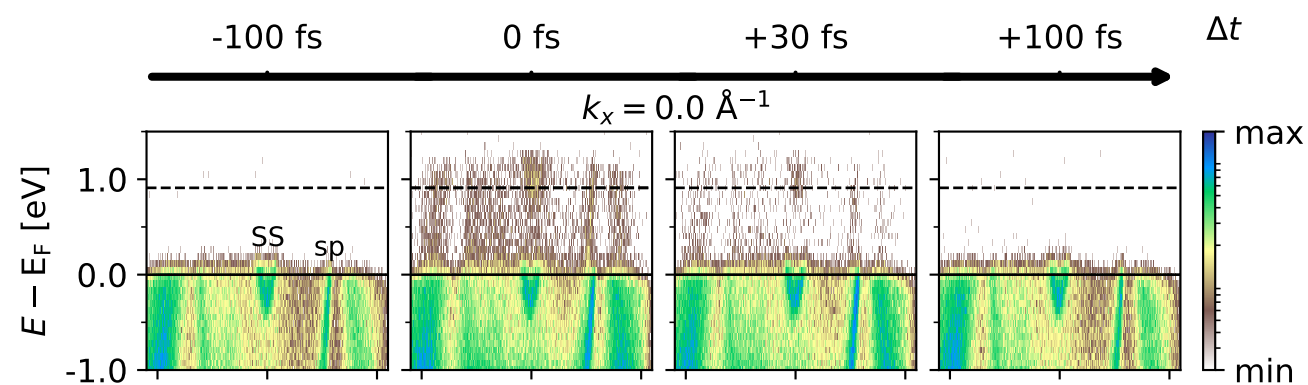

(b)

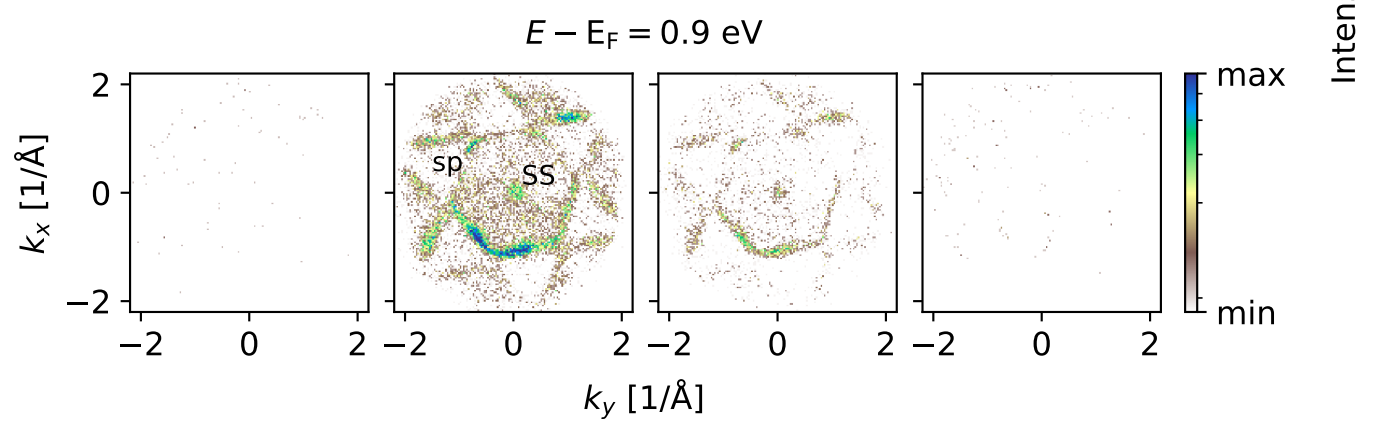

Figure D.2.: Temporal evolution of the sideband yield for selected $\Delta t$. (a) The $\left(E, k_{y}\right)$-resolved spectra are retrieved by slicing the experimentally obtained three-dimensional data set in $k_{y}$ direction and integrating over a $0.13 \AA^{-1}$ large region in $k_{x}$ direction. The dashed and solid lines depict the momentum cut shown in (b) and the Fermi level, respectively. (b) Likewise, the data can be plotted in a $\left(k_{x}, k_{y}\right)$-resolved manner. The integration time for each $\Delta t$ is $15 \mathrm{~min}$. Sideband yield is maximum in temporal overlap and follows the electric field strength of the driving laser field. Taken from [79].

From this data, the temporal and the energetic resolution of the experiment is estimated to $44 \pm 2 \mathrm{fs}$ (FWHM of the cross-correlation) and $\approx 200 \mathrm{meV}$, respectively (see Fig. D.3). 

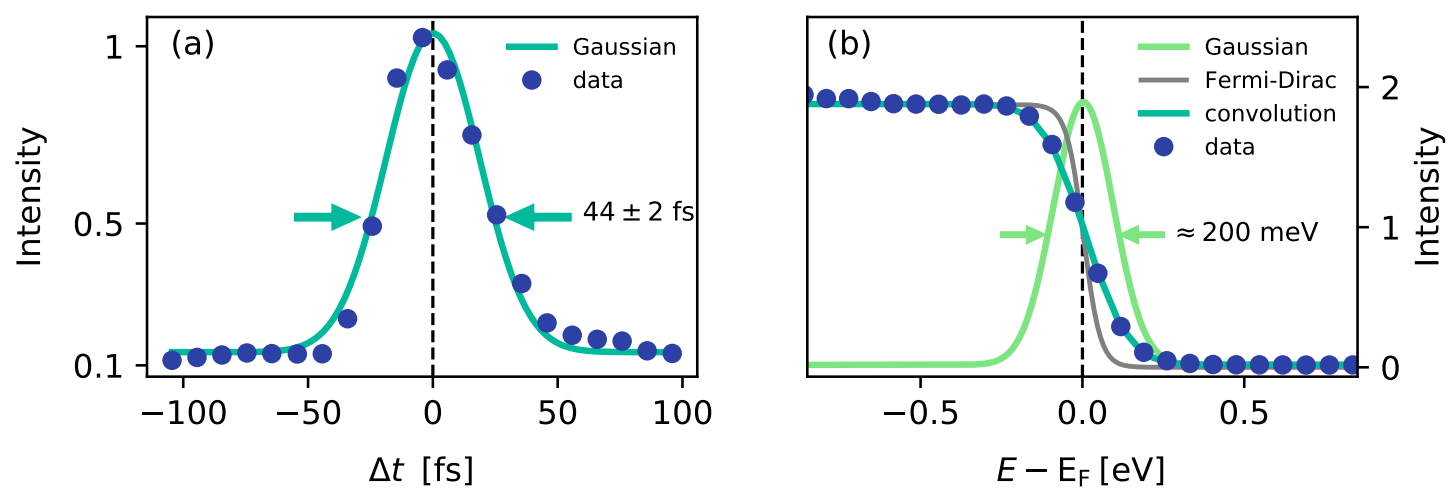

Figure D.3.: Time- and energy-resolution of the time-resolved momentum microscopy experiment. (a) The time-resolution is extracted in a cross correlation experiment by considering the photoemission yield of the first-order sideband following instantaneously the applied electric field strength of the driving laser. A Gaussian fit to the data yields a FWHM of $44 \pm 2$ fs. (b) The energy resolution is extracted by fitting the convolution of a Fermi-Dirac distribution (300 K) and a Gaussian broadening to the measured Fermi edge of $\mathrm{Au}(111)$ (for the $\Delta t=-100$ fs case). The FWHM of the Gaussian broadening is in the range of $\approx 200 \mathrm{meV}$. Details on the time- and energy-resolution of the experiment are discussed in chapter 4. Taken from [79].

\section{D.3. Details on the data analysis and background treatment}

\section{D.3.1. Contributions to the photoelectron background signal}

The HHG beamline is optimized for EUV light with $\hbar \omega_{\text {EUV }}=26.5 \mathrm{eV}\left(11^{\text {th }}\right.$ harmonic). However, as detailed in chapter 4 and Ref. [78, the extinction ratio to the neighboring $13^{\text {th }}$-harmonic is estimated to $1: 470$. In consequence, spectral contributions of the $13^{\text {th }}$-harmonic have to be carefully considered in the evaluations presented in Fig. 6.4 6.5 of the main text. In Fig. D.4, energy distribution curves (EDCs) are shown for the nearly p- and s-polarized driving light. In addition, an EDC obtained away from temporal overlap of the two-color light field is shown ( $\Delta t=-100 \mathrm{fs}$; scaled to match the intensity). The EDC are obtained by integrating the photoelectron counts over the full photoemission horizon. The dressing of the $\mathrm{Au}(111)$ band structure is evident for nearly p-polarized light, whereas in the s-polarized case, no evidence of sidebands is observed. The grey shaded area depicts the integration area used in the analysis in Fig. 6.4 of the main text for the first $\left(\mathrm{n}_{1}\right.$, $\left.E-\mathrm{E}_{\mathrm{F}}=0.9-1.2 \mathrm{eV}\right)$ and the zero-order sideband $\left(\mathrm{n}_{0}, E-\mathrm{E}_{\mathrm{F}}=-0.3-0 \mathrm{eV}\right)$.

Residual photoelectron counts that mainly limit the resolution are induced in 
linear photoemission by the $13^{\text {th }}$ harmonic. These photoelectron counts dominate the measured signal for energies higher than $E-\mathrm{E}_{\mathrm{F}}>1.2 \mathrm{eV}$, as is evident by the nearly overlapped EDCs in Fig. D.4. The handling of this background counts in the analysis of the main text is discussed in the next section.

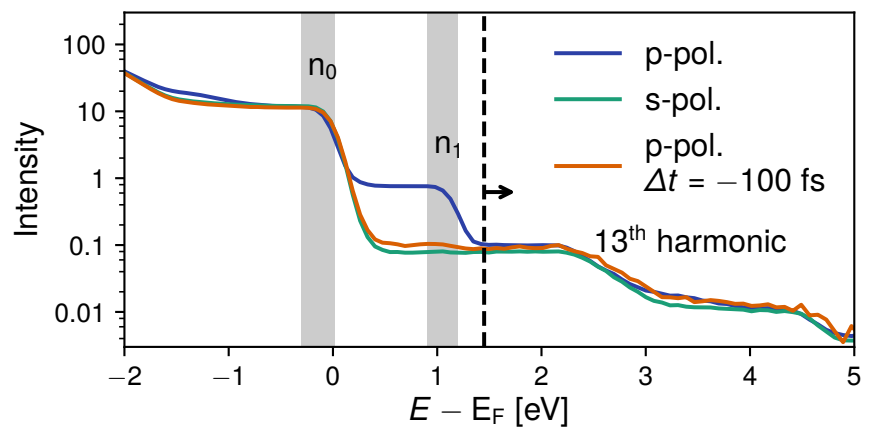

Figure D.4.: Momentum integrated energy distribution spectra for p- and s-polarized driving laser pulses; in addition, an EDC is shown for $\Delta t=-100 \mathrm{fs}$ (scaled) where no sidebands are expected. The integration windows for the zero- and first-order sidebands used in Fig. 6.4 of the main text are indicated by grey vertical areas. For, $E-\mathrm{E}_{\mathrm{F}}>1.2 \mathrm{eV}$, major photoelectron counts are induced by linear photoemission with the $13^{\text {th }}$ harmonic. Taken from [79].

\section{D.3.2. Intensity normalization}

In Fig. 6.4 (c) of the main text, the $\theta_{k}$ dependence of the normalized sideband yield $I_{\text {norm. }}\left(\theta_{k}\right)$ of the SS band and the sp transition are calculated as follows

$$
I_{\text {norm. }}\left(\theta_{k}\right)=\frac{\left[I_{\mathrm{n}_{1}}^{p}\left(\theta_{k}\right)-I_{13^{\text {th }}}^{s}\left(\theta_{k}\right)\right] / I_{\mathrm{n}_{0}}^{s}\left(\theta_{k}\right)}{\left[I_{\mathrm{n}_{1}}^{p}\left(\theta_{k, 0}\right)-I_{13^{\mathrm{th}}}^{s}\left(\theta_{k, 0}\right)\right] / I_{\mathrm{n}_{0}}^{s}\left(\theta_{k, 0}\right)} .
$$

In this data handling, the initial observation is used that s-polarized driving light does not generate sidebands and can thus be used as a reference to normalize the p-polarized case that we are interested in. The momentum maps are divided into areas as shown in Fig. D.5 (a) and all counts are integrated in this area to generate the respective intensity $I\left(\theta_{k}\right)$. First, contributions from the 13 th harmonic (see section D.3.1 are considered by subtracting $I_{13^{\text {th }}}^{s}$ from the first-order sideband yield $I_{\mathrm{n}_{1}}^{p}\left(\theta_{k}\right) \cdot I_{13^{\text {th }}}^{s}$ is generated by integrating all photoelectron counts in a $\left(E-\mathrm{E}_{\mathrm{F}}=\right.$ $0.9-1.2 \mathrm{eV}$ ) interval for the s-polarized measurement [see Fig. D.5 (b)]. Second, this difference is normalized onto the $\theta_{k}$-dependent intensity $I_{\mathrm{n}_{0}}^{s}\left(\theta_{k}\right)$ of the undressed system [see Fig. D.5 (c)]. This normalization takes care of the initial asymmetry of the undriven momentum distributions due to alignment of the microscope and matrix element effects. The denominator of Eq. D.1 then references all intensities to 
the area with highest sideband yield $\left(\theta_{k, 0}\right.$ is the area in Fig. D.5 (a) labelled with an arrow). $I_{\text {norm. }}\left(\theta_{k}\right)=1$ then implies that the first-order sideband is a perfect replica of the zero-order-sideband.
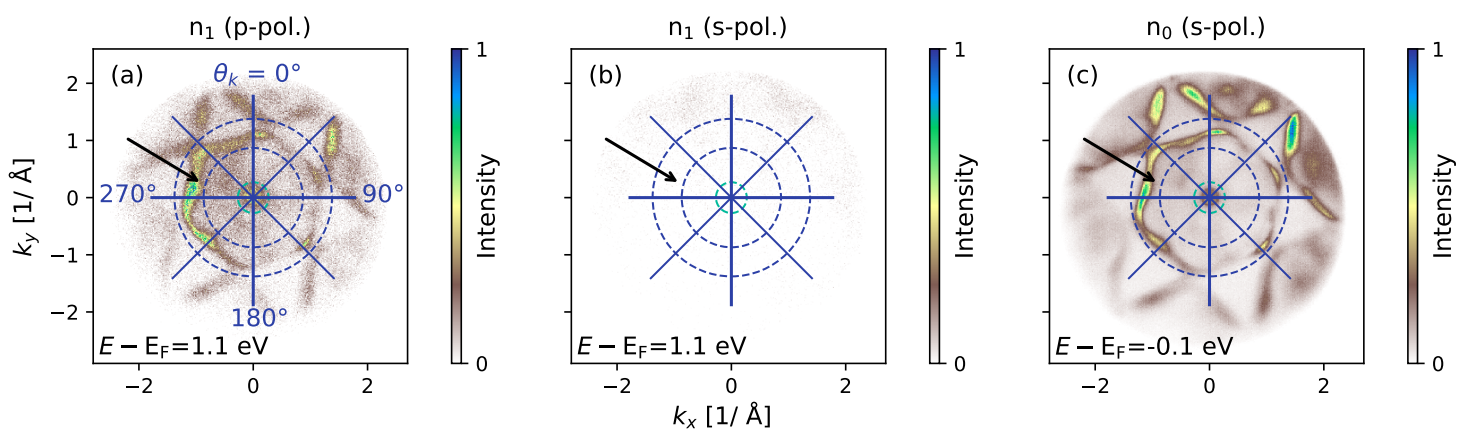

Figure D.5.: Momentum maps that are used in the analysis in Fig. 6.4 (c) of the main text. We divide the momentum maps into areas as indicated by the blue lines and integrate all photoelectrons within the region of interest. The reference area is indicated by an arrow. From (a) to (c), we show the first-order sideband (p-polarized driving), the (non-existing) first order sideband (s-polarized driving), and the zero-order sideband (spolarized driving). The energy of the momentum maps is given in the figure. Taken from [79]. 


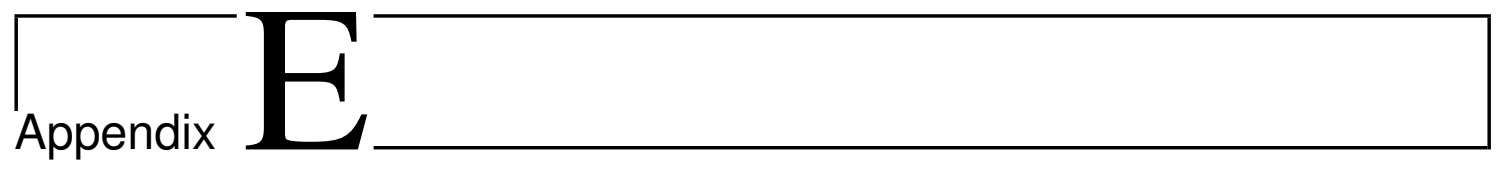

\section{List of publications}

Publications directly related to this thesis:

1. M. Keunecke, C. Möller, D. Schmitt, H. Nolte, G. S. M. Jansen, M. Reutzel, M. Gutberlet, G. Halasi, D. Steil, S. Steil, and S. Mathias:

Time-resolved momentum microscopy with a $1 \mathrm{MHz}$ high-harmonic extreme ultraviolet beamline

Review of Scientific Instruments 91 (6) (2020)

DOI: $10.1063 / 5.0006531$

2. M. Keunecke, M. Reutzel, D. Schmitt, A. Osterkorn, T. A. Mishra, C. Möller, W. Bennecke, G. S. M. Jansen, D. Steil, S. R. Manmana, S. Steil, S. Kehrein, and S. Mathias:

Electromagnetic dressing of the electron energy spectrum of $\mathrm{Au}(111)$ at high momenta

Physical Review B 102.16 (2020): 161403

DOI:10.1103/PhysRevB.102.161403

3. M. Keunecke, D. Schmitt, M. Reutzel, M. Weber, C. Möller, G. S. M. Jansen, T. A. Mishra, A. Osterkorn, W. Bennecke, K. Pierz, H.W. Schumacher, D.M. Pakdehi, D. Steil, S. R. Manmana, S. Steil, S. Kehrein, H.C. Schneider, and S. Mathias:

Direct Access to Auger Recombination in Graphene arXiv (2020): 2012.01256

Other publications:

4. P. Thiessen, V. Roddatis, F. Rieger, A. Belenchuk, M. Keunecke, V. Moshnyaga, and Ch. Jooss:

Effect of charge ordering on crossplane thermal conductivity in correlated perovskite oxide superlattices

Physical Review B 98.19 (2018): 195114.

DOI: 10.1103/PhysRevB.98.195114 
5. M. Keunecke, F. Lyzwa, D. Schwarzbach, V. Roddatis, N. Gauquelin, K. Müller-Caspary, J. Verbeeck, S. J. Callori, F. Klose, M. Jungbauer, and V. Moshnyaga:

High- $\mathrm{T}_{\mathrm{C}}$ Interfacial Ferromagnetism in $\mathrm{SrMnO}_{3} / \mathrm{LaMnO}_{3}$ Superlattices Advanced Functional Materials 30.18 (2020): 1808270

DOI:10.1002/adfm.201808270

6 G. S. M. Jansen, M. Keunecke, M. Düvel, C. Möller, D. Schmitt, W. Bennecke, F. J. S. Kappert, D. Steil, D. R. Luke, S. Steil, and S. Mathias:

Efficient orbital imaging based on ultrafast momentum microscopy and sparsitydriven phase retrieval

New Journal of Physics 22 (6) (2020)

DOI:10.1088/1367-2630/ab8aae

7 V. Roddatis, J.P. Bange, S. Lopatin, M. Keunecke, D. Meyer, D. Metternich, U. Roß, I.V. Maznichenko, S. Ostanin, I. Mertig, V. Radisch, R. Egoavil, I. Lazić, V. Moshnyaga, and H. Ulrichs:

$\mathrm{MnO}_{6}$ Octahedral Tilt Control of Emergent Phenomena at $\mathrm{LaMnO}_{3} / \mathrm{SrMnO}_{3}$ Interfaces

arXiv (2020): 2009.14532 


\section{Acknowledgment}

In this part, I want to thank all the people that contributed, in one way or another, to the completion of this thesis.

- First, I would like to thank my family for their support, which made it possible for me to study physics in Göttingen. Even though they were not very convinced by the idea.

- I want to thank Stefan for the opportunity to work on this project and the resulting involvement in building up a lab. This was a very nice experience. Additionally, I am grateful for the countless discussions over various topics, the insights into being/becoming a professor, and the possibility to attend several conferences (especially the one in China).

- I also wish to thank Vasily, who guided me already through my master- and bachelor thesis. It is always a pleasure working him and discussing various topics. I want to thank him also for his kind words and his efforts to keep the spirits up.

- I want to thank Prof. Dr. Claus Ropers for taking the job as second reviewer and Prof. Dr. Thomas Weitz, Prof. Dr. Stefan Kehrein, and PD Dr. Martin Wenderoth for taking part in the examination board.

- Special thanks go to David who helped a lot in building up the MoMi setup and keeping it running. I am grateful for several measurement runs over days/nights/weekends, various discussions, and for proofreading this thesis.

- I also want to thank Christina for taking care of the HHG beamline, her effort in keeping a nice lab atmosphere, and proofreading of parts in this thesis.

- I would like to thank Marcel for countless comments on figures, how to write a paper, and how to present data so others can follow. Working on the publications together was sometimes exhausting, but in the end it was very good. I also like the various discussions, where I learn a lot. I am grateful for your proofreading of this thesis and the regularly jokes. 
- Special thanks go to Carsten, Uta, Kai, Katrin and Simon for their technical support and also the nice working atmosphere. Without their help the MoMi setup would not have been possible. I also want to thank Hartmut Eichenberg, Bastian Beyland and the central workshop for providing various parts for the setup (sometimes urgently, if required).

- I want to thank Daniel, Sabine and Matthijs for their help and comments on many different topics.

- I am grateful for the help I got from Michaela Mecke and Finn Krummacker concerning all organizational stuff.

- I would like to thank Alexander, Tridev, Salvatore, Stefan Kehrein, Marius Weber and Hans-Christian Schneider for interesting discussions and theory support.

- I wish to thank my office mate Tim and my pre-corona office mates Cinja and Marco for keeping up the good spirit and the overall nice atmosphere.

- I want to thank all other members of the AG Mathias, especially the various master- and bachelor students, who helped in different topics throughout this thesis and kept a good atmosphere.

- In the end, I would like to thank all other members of the institute for the nice atmosphere. I have been here for quite some time and have found many friends along the way. 